\title{
Computer-Aided Molecular Design of Bis-phosphine Oxide
}

\section{Lanthanide Extractants}

Billy W. McCann ${ }^{1}$, Nuwan De Silva ${ }^{2}$, Theresa L. Windus ${ }^{2}$, Mark S. Gordon ${ }^{2}$, Bruce A. Moyer $^{1}$, Vyacheslav S. Bryantsev*1 ${ }^{1}$, and Benjamin P. Hay*3

${ }^{1}$ Oak Ridge National Laboratory, Chemical Sciences Division, 1 Bethel Valley Rd., Oak Ridge, Tennessee 37831-6119, United States

${ }^{2}$ Department of Chemistry, Iowa State University, and Ames Laboratory, Ames, Iowa 50011-3111, United States

${ }^{3}$ Supramolecular Design Institute, 127 Chestnut Hill Rd., Oak Ridge, Tennessee 378307185, United States

*Corresponding Authors:

Email: bryantsevv@ornl.gov, hayben@,comcast.net

Electronic Supporting Information. HostDesigner input files, modified MM3 parameters, basis for MM3 parameterization, potential diphosphoranedione architectures, atomic coordinates for MM3 optimized metal complex and global minimum geometries for 1-65, metal size preference scans for $\mathbf{1 - 6 5}$, atomic coordinates and absolute energies for all DFT optimized geometries, and full citation for reference 35(a). 


\section{(1) HostDesigner input files used to obtain reported results:}

\section{Control file:}

OVER hosta=overphos AND

drivea numkeep $=3000$ numview $=3000$ AND

maxrmsd=1.0 tightness $=0.50$ maxnrot $=5$ AND

linklib=/Users/hay/bin/hd_dir/LIBRARY_HC_syn

Input fragment file:

Bis-phosphine oxide + Gd(III)

\begin{tabular}{|c|c|c|c|c|c|c|c|c|c|c|c|c|c|}
\hline $\mathrm{P}$ & & 1 & \multicolumn{2}{|c|}{-1.370500} & & \multicolumn{2}{|c|}{0.922900} & 0.000000 & 153 & 2 & 3 & 4 & 9 \\
\hline O & & 2 & \multicolumn{2}{|c|}{-1.050500} & & \multicolumn{2}{|c|}{-0.532300} & 0.000000 & 7 & 1 & 23 & & \\
\hline C & & 3 & \multicolumn{2}{|c|}{-2.327700} & & \multicolumn{2}{|c|}{1.457300} & -1.428800 & 1 & 1 & 10 & 11 & 12 \\
\hline C & & 4 & \multicolumn{2}{|c|}{-2.330700} & & \multicolumn{2}{|c|}{1.456400} & 1.427100 & 1 & 1 & 13 & 14 & 15 \\
\hline $\mathrm{P}$ & & 5 & \multicolumn{2}{|c|}{1.370600} & & \multicolumn{2}{|c|}{0.920900} & 0.000400 & 153 & 6 & 7 & 8 & 16 \\
\hline O & & 6 & \multicolumn{2}{|c|}{1.045700} & & \multicolumn{2}{|c|}{-0.533300} & -0.000400 & 7 & 5 & 23 & & \\
\hline C & & 7 & \multicolumn{2}{|c|}{2.332500} & & \multicolumn{2}{|c|}{1.450700} & 1.427800 & 1 & 5 & 17 & 18 & 19 \\
\hline C & & 8 & \multicolumn{2}{|c|}{2.329700} & & \multicolumn{2}{|c|}{1.452600} & -1.428100 & 1 & 5 & 20 & 21 & 22 \\
\hline $\mathrm{H}$ & & 9 & \multicolumn{2}{|c|}{0.080300} & & \multicolumn{2}{|c|}{1.986200} & 0.002400 & 5 & 1 & & & \\
\hline $\mathrm{H}$ & & 10 & \multicolumn{2}{|c|}{-3.294000} & & \multicolumn{2}{|c|}{0.913700} & -1.463200 & 5 & 3 & & & \\
\hline $\mathrm{H}$ & & 11 & \multicolumn{2}{|c|}{-1.766700} & & \multicolumn{2}{|c|}{1.245600} & -2.362000 & 5 & 3 & & & \\
\hline $\mathrm{H}$ & & 12 & \multicolumn{2}{|c|}{-2.531500} & & \multicolumn{2}{|c|}{2.546100} & -1.372900 & 5 & 3 & & & \\
\hline $\mathrm{H}$ & & 13 & \multicolumn{2}{|c|}{-3.297000} & & \multicolumn{2}{|c|}{0.912900} & 1.459200 & 5 & 4 & & & \\
\hline $\mathrm{H}$ & & 14 & \multicolumn{2}{|c|}{-2.534300} & & & 545300 & 1.371300 & 5 & 4 & & & \\
\hline $\mathrm{H}$ & & 15 & -1 & 771700 & & & 244300 & 2.361300 & 5 & 4 & & & \\
\hline $\mathrm{H}$ & & 16 & -0 . & 076700 & & & 989100 & 0.002100 & 5 & 5 & & & \\
\hline $\mathrm{H}$ & & 17 & 3. & 297000 & & & 903900 & 1.459700 & 5 & 7 & & & \\
\hline $\mathrm{H}$ & & 18 & 1 . & 772600 & & & 240000 & 2.362000 & 5 & 7 & & & \\
\hline $\mathrm{H}$ & & 19 & 2 . & 539700 & & & 538800 & 1.372500 & 5 & 7 & & & \\
\hline $\mathrm{H}$ & & 20 & 3. & 294100 & & & 906100 & -1.462700 & 5 & 8 & & & \\
\hline $\mathrm{H}$ & & 21 & 2 . & 536900 & & & 540700 & -1.371700 & 5 & 8 & & & \\
\hline $\mathrm{H}$ & & 22 & & 768000 & & & 243400 & -2.361400 & 5 & 8 & & & \\
\hline $\begin{array}{l}G \\
2\end{array}$ & & 23 & -0 . & 006400 & & -2 & 546100 & 0.000300 & 345 & 2 & 6 & & \\
\hline 16 & C & 0 & & & & & & & & & & & \\
\hline 9 & $\mathrm{C}$ & 0 & & & & & & & & & & & \\
\hline D & & & & & & & & & & & & & \\
\hline A & I & 0.0 & 40.0 & 5.0 & 2 & 23 & 6 & & & & & & \\
\hline D & I & -90.0 & 90.0 & 15.0 & 2 & 1 & & & & & & & \\
\hline D & I & -90.0 & 90.0 & 15.0 & 6 & 5 & & & & & & & \\
\hline A & I & -14.0 & 14.0 & 7.0 & 23 & 2 & 1 & & & & & & \\
\hline A & I & -14.0 & 14.0 & 7.0 & 23 & 6 & 5 & & & & & & \\
\hline
\end{tabular}

(2) Modified MM3 parameter set read in as 'added parameters' prior to calculation with the PCModel software. 


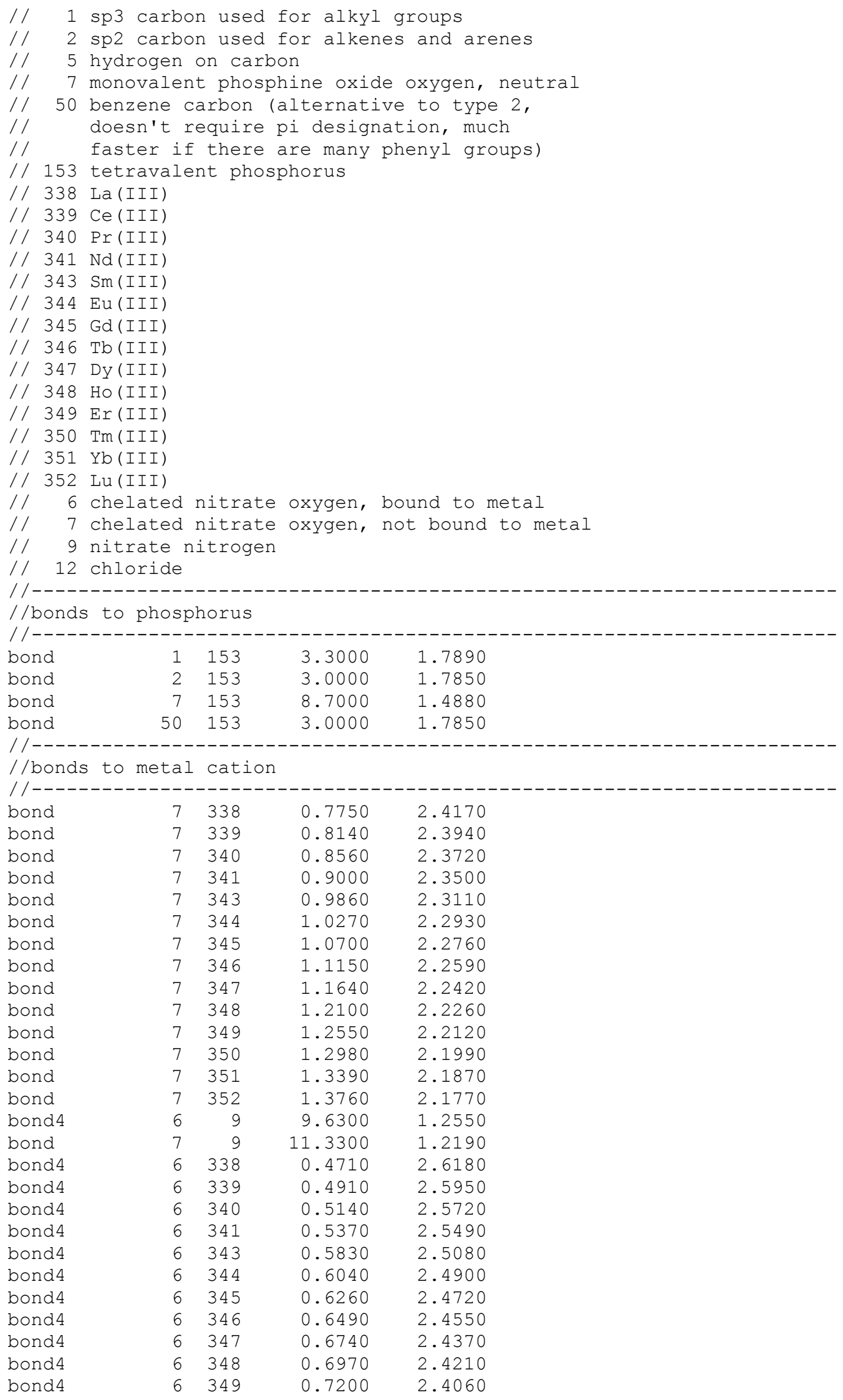




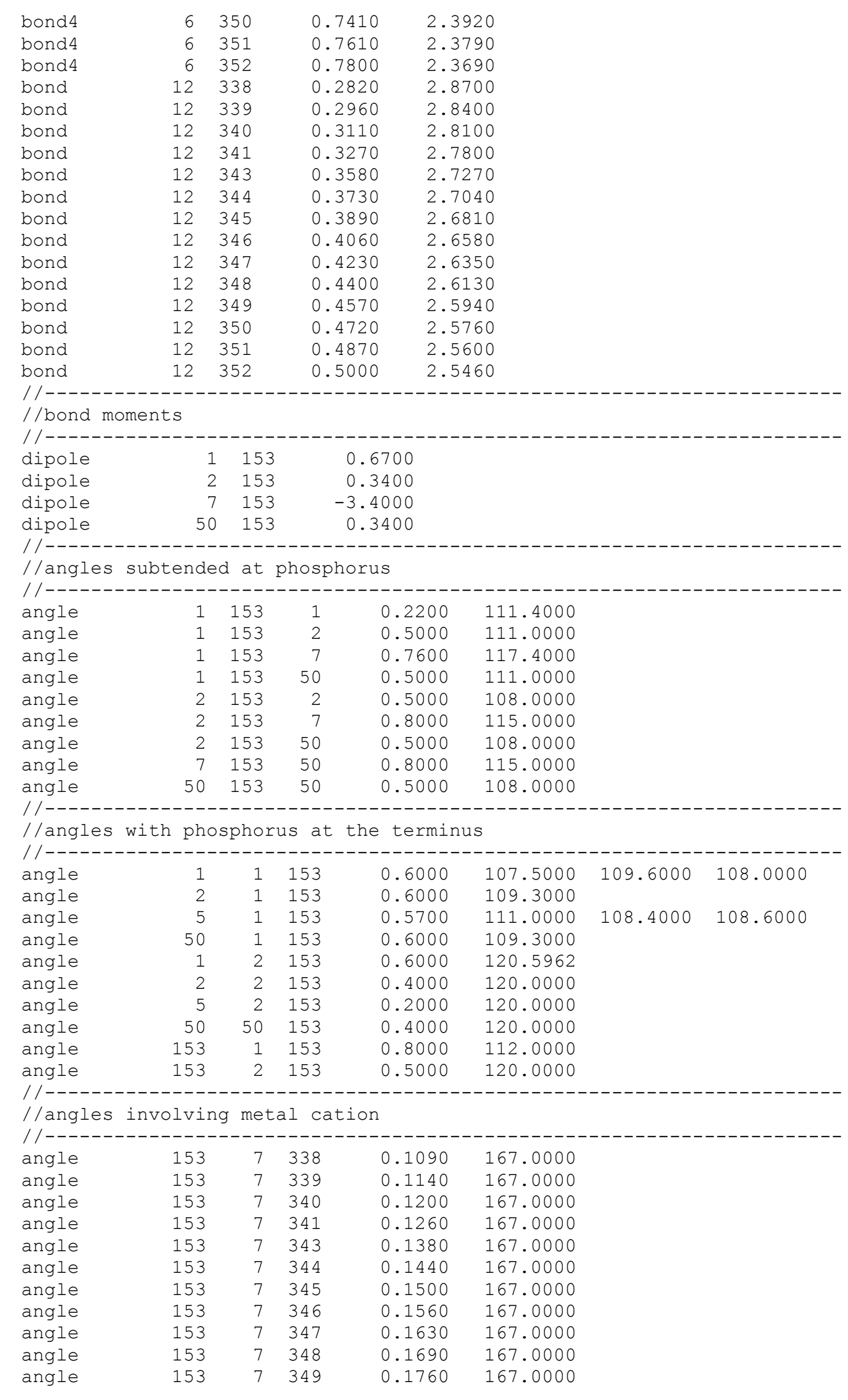




\begin{tabular}{|c|c|c|c|c|c|c|c|c|c|}
\hline angle & 153 & \multicolumn{2}{|c|}{350} & 0.1820 & 167.0000 & & & & \\
\hline angle & 153 & \multicolumn{2}{|c|}{351} & 0.1870 & 167.0000 & & & & \\
\hline angle & 153 & \multicolumn{2}{|c|}{352} & 0.1930 & 167.0000 & & & & \\
\hline angle4 & 6 & 9 & 6 & 0.7000 & 116.8000 & & & & \\
\hline angle & 6 & 9 & 7 & 0.7000 & 121.6000 & & & & \\
\hline angle4 & 9 & 6 & 38 & 0.0000 & 120.0000 & & & & \\
\hline angle4 & 9 & 6 & 39 & 0.0000 & 120.0000 & & & & \\
\hline angle4 & 9 & 6 & 40 & 0.0000 & 120.0000 & & & & \\
\hline angle4 & 9 & 6 & 41 & 0.0000 & 120.0000 & & & & \\
\hline angle4 & 9 & 6 & 43 & 0.0000 & 120.0000 & & & & \\
\hline angle4 & 9 & 6 & 44 & 0.0000 & 120.0000 & & & & \\
\hline angle4 & 9 & 6 & 45 & 0.0000 & 120.0000 & & & & \\
\hline angle4 & 9 & 6 & 46 & 0.0000 & 120.0000 & & & & \\
\hline angle4 & 9 & 6 & 47 & 0.0000 & 120.0000 & & & & \\
\hline angle4 & 9 & 6 & 48 & 0.0000 & 120.0000 & & & & \\
\hline angle4 & 9 & 6 & 49 & 0.0000 & 120.0000 & & & & \\
\hline angle4 & 9 & 6 & 50 & 0.0000 & 120.0000 & & & & \\
\hline angle4 & 9 & 6 & 51 & 0.0000 & 120.0000 & & & & \\
\hline angle4 & 9 & 6 & 52 & 0.0000 & 120.0000 & & & & \\
\hline $\begin{array}{l}\text { //----- } \\
\text { //dihed }\end{array}$ & les & & ed & & & & & & \\
\hline /----- & $-\ldots$ & 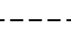 & --- & -- & --------- & 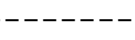 & . & ------- & \\
\hline torsion & 1 & 1 & 153 & 1 & $-0.080+1$ & -0.230 & -2 & 0.330 & +3 \\
\hline torsion & 1 & 1 & 153 & 2 & $-0.080+1$ & -0.230 & -2 & 0.330 & +3 \\
\hline torsion & 1 & 1 & 153 & 7 & $0.000+1$ & 0.000 & -2 & 0.330 & +3 \\
\hline torsion & 1 & 1 & 153 & 50 & $-0.080+1$ & -0.230 & -2 & 0.330 & +3 \\
\hline torsion & 2 & 1 & 153 & 1 & $0.280+1$ & 0.000 & -2 & 0.480 & +3 \\
\hline torsion & 2 & 1 & 153 & 2 & $0.280+1$ & 0.000 & -2 & 0.480 & +3 \\
\hline torsion & 2 & 1 & 153 & 7 & $0.280+1$ & 0.000 & -2 & 0.480 & +3 \\
\hline torsion & 2 & 1 & 153 & 50 & $0.280+1$ & 0.000 & -2 & 0.480 & +3 \\
\hline torsion & 5 & 1 & 153 & 1 & $0.000+1$ & 0.000 & -2 & 0.205 & +3 \\
\hline torsion & 5 & 1 & 153 & 2 & $0.000+1$ & 0.000 & -2 & 0.205 & +3 \\
\hline torsion & 5 & 1 & 153 & 7 & $0.000+1$ & 0.000 & -2 & 0.205 & +3 \\
\hline torsion & 5 & 1 & 153 & 50 & $0.000+1$ & 0.000 & -2 & 0.205 & +3 \\
\hline torsion & 50 & 1 & 153 & 1 & $0.280+1$ & 0.000 & -2 & 0.480 & +3 \\
\hline torsion & 50 & 1 & 153 & 2 & $0.280+1$ & 0.000 & -2 & 0.480 & +3 \\
\hline torsion & 50 & 1 & 153 & 7 & $-0.280+1$ & 0.000 & -2 & 0.480 & +3 \\
\hline torsion & 50 & 1 & 153 & 50 & $0.280+1$ & 0.000 & -2 & 0.480 & +3 \\
\hline torsion & 153 & 1 & 153 & 1 & $0.350+1$ & -0.250 & -2 & 0.400 & +3 \\
\hline torsion & 153 & 1 & 153 & 2 & $0.350+1$ & -0.250 & -2 & 0.400 & +3 \\
\hline torsion & 153 & 1 & 153 & 7 & $-0.350+1$ & 0.250 & -2 & -0.400 & +3 \\
\hline torsion & 153 & 1 & 153 & 50 & $0.350+1$ & -0.250 & -2 & 0.400 & +3 \\
\hline torsion & 1 & 2 & 153 & 1 & $0.000+1$ & 0.000 & -2 & 0.550 & +3 \\
\hline torsion & 1 & 2 & 153 & 2 & $0.000+1$ & 0.000 & -2 & 0.550 & +3 \\
\hline torsion & 1 & 2 & 153 & 7 & $-0.450+1$ & -0.300 & -2 & 0.550 & +3 \\
\hline torsion & 1 & 2 & 153 & 50 & $0.000+1$ & 0.000 & -2 & 0.550 & +3 \\
\hline torsion & 2 & 2 & 153 & 1 & $0.260+1$ & -0.570 & -2 & -0.370 & +3 \\
\hline torsion & 2 & 2 & 153 & 2 & $0.260+1$ & -0.570 & -2 & -0.370 & +3 \\
\hline torsion & 2 & 2 & 153 & 7 & $-0.260+1$ & 0.570 & -2 & -0.370 & +3 \\
\hline torsion & 2 & 2 & 153 & 50 & $0.260+1$ & -0.570 & -2 & -0.370 & +3 \\
\hline torsion & 5 & 2 & 153 & 1 & $-0.260+1$ & -0.130 & -2 & 0.370 & +3 \\
\hline torsion & 5 & 2 & 153 & 2 & $-0.260+1$ & -0.130 & -2 & 0.370 & +3 \\
\hline torsion & 5 & 2 & 153 & 7 & $0.260+1$ & 0.130 & -2 & 0.370 & +3 \\
\hline torsion & 5 & 2 & 153 & 50 & $-0.300+1$ & -0.250 & -2 & 0.370 & +3 \\
\hline torsion & 153 & 2 & 153 & 1 & $0.000+1$ & 0.000 & -2 & 0.000 & +3 \\
\hline torsion & 153 & 2 & 153 & 2 & $0.000+1$ & 0.000 & -2 & 0.000 & +3 \\
\hline torsion & 153 & 2 & 153 & 7 & $0.000+1$ & 0.000 & -2 & 0.000 & +3 \\
\hline torsion & 153 & 2 & 153 & 50 & $0.000+1$ & 0.000 & -2 & 0.000 & +3 \\
\hline torsion & 50 & 50 & 153 & 1 & $0.260+1$ & -0.570 & -2 & -0.370 & +3 \\
\hline torsion & 50 & 50 & 153 & 2 & $0.260+1$ & -0.570 & -2 & -0.370 & +3 \\
\hline torsion & 50 & 50 & 153 & 7 & $-0.260+1$ & 0.570 & -2 & -0.370 & +3 \\
\hline torsion & 50 & 50 & 153 & 50 & $0.260+1$ & -0.570 & -2 & -0.370 & +3 \\
\hline
\end{tabular}


//dihedral angles with phosphorus at the terminus

\begin{tabular}{|c|c|c|c|c|c|c|c|c|c|c|}
\hline torsion & 1 & 1 & 1 & 153 & -0.560 & +1 & -0.260 & -2 & 0.150 & +3 \\
\hline torsion & 2 & 1 & 1 & 153 & -2.500 & +1 & 0.000 & -2 & 1.000 & +3 \\
\hline torsion & 5 & 1 & 1 & 153 & 0.000 & +1 & 0.000 & -2 & 0.280 & +3 \\
\hline torsion & 50 & 1 & 1 & 153 & -2.500 & +1 & 0.000 & -2 & 1.000 & +3 \\
\hline torsion & 153 & 1 & 1 & 153 & 0.300 & +1 & -0.300 & -2 & -1.400 & +3 \\
\hline torsion & 1 & 1 & 2 & 153 & 0.000 & +1 & 0.600 & -2 & 0.430 & +3 \\
\hline torsion & 2 & 1 & 2 & 153 & -0.420 & +1 & -0.340 & -2 & 2.530 & +3 \\
\hline torsion & 5 & 1 & 2 & 153 & 0.000 & +1 & 0.000 & -2 & 0.430 & +3 \\
\hline torsion & 50 & 1 & 2 & 153 & -0.420 & +1 & -0.340 & -2 & 2.530 & +3 \\
\hline torsion & 153 & 1 & 2 & 1 & -0.640 & +1 & -0.110 & -2 & 0.970 & +3 \\
\hline torsion & 153 & 1 & 2 & 2 & -0.640 & +1 & -0.780 & -2 & -0.300 & +3 \\
\hline torsion & 153 & 1 & 2 & 5 & 0.640 & +1 & -0.400 & -2 & 0.300 & +3 \\
\hline torsion & 153 & 1 & 2 & 153 & 0.000 & +1 & 0.000 & -2 & 0.000 & +3 \\
\hline torsion & 1 & 2 & 2 & 153 & 0.810 & +1 & 8.300 & -2 & 0.950 & +3 \\
\hline torsion & 2 & 2 & 2 & 153 & 0.810 & +1 & 8.300 & -2 & -0.950 & +3 \\
\hline torsion & 5 & 2 & 2 & 153 & 0.000 & +1 & 10.000 & -2 & 0.000 & +3 \\
\hline torsion & 153 & 2 & 2 & 153 & -0.300 & +1 & 9.500 & -2 & 0.000 & +3 \\
\hline torsion & 153 & 1 & 50 & 5 & 0.640 & +1 & -0.400 & -2 & 0.300 & +3 \\
\hline torsion & 153 & 1 & 50 & 50 & -0.640 & +1 & -0.780 & -2 & -0.300 & +3 \\
\hline torsion & 1 & 50 & 50 & 153 & 0.810 & +1 & 8.300 & -2 & 0.950 & +3 \\
\hline torsion & 2 & 50 & 50 & 153 & 0.810 & +1 & 8.300 & -2 & -0.950 & +3 \\
\hline torsion & 5 & 50 & 50 & 153 & 0.000 & +1 & 10.000 & -2 & 0.000 & +3 \\
\hline torsion & 50 & 50 & 50 & 153 & 0.810 & +1 & 8.300 & -2 & -0.950 & +3 \\
\hline torsion & 153 & 50 & 50 & 153 & -0.300 & +1 & 9.500 & -2 & 0.000 & +3 \\
\hline \multirow{2}{*}{\multicolumn{11}{|c|}{ //dihedral angles involving metal cation }} \\
\hline & & & & & & & & & & \\
\hline torsion & 338 & 7 & 153 & 1 & 0.000 & +1 & 0.000 & -2 & 0.000 & +3 \\
\hline torsion & 339 & 7 & 153 & 1 & 0.000 & +1 & 0.000 & -2 & 0.000 & +3 \\
\hline torsion & 340 & 7 & 153 & 1 & 0.000 & +1 & 0.000 & -2 & 0.000 & +3 \\
\hline torsion & 341 & 7 & 153 & 1 & 0.000 & +1 & 0.000 & -2 & 0.000 & +3 \\
\hline torsion & 343 & 7 & 153 & 1 & 0.000 & +1 & 0.000 & -2 & 0.000 & +3 \\
\hline torsion & 344 & 7 & 153 & 1 & 0.000 & +1 & 0.000 & -2 & 0.000 & +3 \\
\hline torsion & 345 & 7 & 153 & 1 & 0.000 & +1 & 0.000 & -2 & 0.000 & +3 \\
\hline torsion & 346 & 7 & 153 & 1 & 0.000 & +1 & 0.000 & -2 & 0.000 & +3 \\
\hline torsion & 347 & 7 & 153 & 1 & 0.000 & +1 & 0.000 & -2 & 0.000 & +3 \\
\hline torsion & 348 & 7 & 153 & 1 & 0.000 & +1 & 0.000 & -2 & 0.000 & +3 \\
\hline torsion & 349 & 7 & 153 & 1 & 0.000 & +1 & 0.000 & -2 & 0.000 & +3 \\
\hline torsion & 350 & 7 & 153 & 1 & 0.000 & +1 & 0.000 & -2 & 0.000 & +3 \\
\hline torsion & 351 & 7 & 153 & 1 & 0.000 & +1 & 0.000 & -2 & 0.000 & +3 \\
\hline torsion & 352 & 7 & 153 & 1 & 0.000 & +1 & 0.000 & -2 & 0.000 & +3 \\
\hline torsion & 338 & 7 & 153 & 2 & 0.000 & +1 & 0.000 & -2 & 0.000 & +3 \\
\hline torsion & 339 & 7 & 153 & 2 & 0.000 & +1 & 0.000 & -2 & 0.000 & +3 \\
\hline torsion & 340 & 7 & 153 & 2 & 0.000 & +1 & 0.000 & -2 & 0.000 & +3 \\
\hline torsion & 341 & 7 & 153 & 2 & 0.000 & +1 & 0.000 & -2 & 0.000 & +3 \\
\hline torsion & 343 & 7 & 153 & 2 & 0.000 & +1 & 0.000 & -2 & 0.000 & +3 \\
\hline torsion & 344 & 7 & 153 & 2 & 0.000 & +1 & 0.000 & -2 & 0.000 & +3 \\
\hline torsion & 345 & 7 & 153 & 2 & 0.000 & +1 & 0.000 & -2 & 0.000 & +3 \\
\hline torsion & 346 & 7 & 153 & 2 & 0.000 & +1 & 0.000 & -2 & 0.000 & +3 \\
\hline torsion & 347 & 7 & 153 & 2 & 0.000 & +1 & 0.000 & -2 & 0.000 & +3 \\
\hline torsion & 348 & 7 & 153 & 2 & 0.000 & +1 & 0.000 & -2 & 0.000 & +3 \\
\hline torsion & 349 & 7 & 153 & 2 & 0.000 & +1 & 0.000 & -2 & 0.000 & +3 \\
\hline torsion & 350 & 7 & 153 & 2 & 0.000 & +1 & 0.000 & -2 & 0.000 & +3 \\
\hline torsion & 351 & 7 & 153 & 2 & 0.000 & +1 & 0.000 & -2 & 0.000 & +3 \\
\hline torsion & 352 & 7 & 153 & 2 & 0.000 & +1 & 0.000 & -2 & 0.000 & +3 \\
\hline torsion & 338 & 7 & 153 & 50 & 0.000 & +1 & 0.000 & -2 & 0.000 & +3 \\
\hline torsion & 339 & 7 & 153 & 50 & 0.000 & +1 & 0.000 & -2 & 0.000 & +3 \\
\hline torsion & 340 & 7 & 153 & 50 & 0.000 & +1 & 0.000 & -2 & 0.000 & +3 \\
\hline torsion & 341 & 7 & 153 & 50 & 0.000 & +1 & 0.000 & -2 & 0.000 & +3 \\
\hline torsion & 343 & 7 & 153 & 50 & 0.000 & +1 & 0.000 & -2 & 0.000 & +3 \\
\hline torsion & 344 & 7 & 153 & 50 & 0.000 & +1 & 0.000 & -2 & 0.000 & +3 \\
\hline
\end{tabular}




\begin{tabular}{|c|c|c|c|c|c|c|c|c|c|c|}
\hline torsion & 345 & 7 & 153 & 50 & 0.000 & +1 & 0.000 & -2 & 0.000 & +3 \\
\hline torsion & 346 & 7 & 153 & 50 & 0.000 & +1 & 0.000 & -2 & 0.000 & +3 \\
\hline torsion & 347 & 7 & 153 & 50 & 0.000 & +1 & 0.000 & -2 & 0.000 & +3 \\
\hline torsion & 348 & 7 & 153 & 50 & 0.000 & +1 & 0.000 & -2 & 0.000 & +3 \\
\hline torsion & 349 & 7 & 153 & 50 & 0.000 & +1 & 0.000 & -2 & 0.000 & +3 \\
\hline torsion & 350 & 7 & 153 & 50 & 0.000 & +1 & 0.000 & -2 & 0.000 & +3 \\
\hline torsion & 351 & 7 & 153 & 50 & 0.000 & +1 & 0.000 & -2 & 0.000 & +3 \\
\hline torsion & 352 & 7 & 153 & 50 & 0.000 & +1 & 0.000 & -2 & 0.000 & +3 \\
\hline torsion & 338 & 6 & 9 & 6 & 0.000 & +1 & 1.000 & -2 & 0.000 & +3 \\
\hline torsion & 339 & 6 & 9 & 6 & 0.000 & +1 & 1.000 & -2 & 0.000 & +3 \\
\hline torsion & 340 & 6 & 9 & 6 & 0.000 & +1 & 1.000 & -2 & 0.000 & +3 \\
\hline torsion & 341 & 6 & 9 & 6 & 0.000 & +1 & 1.000 & -2 & 0.000 & +3 \\
\hline torsion & 343 & 6 & 9 & 6 & 0.000 & +1 & 1.000 & -2 & 0.000 & +3 \\
\hline torsion & 344 & 6 & 9 & 6 & 0.000 & +1 & 1.000 & -2 & 0.000 & +3 \\
\hline torsion & 345 & 6 & 9 & 6 & 0.000 & +1 & 1.000 & -2 & 0.000 & +3 \\
\hline torsion & 346 & 6 & 9 & 6 & 0.000 & +1 & 1.000 & -2 & 0.000 & +3 \\
\hline torsion & 347 & 6 & 9 & 6 & 0.000 & +1 & 1.000 & -2 & 0.000 & +3 \\
\hline torsion & 348 & 6 & 9 & 6 & 0.000 & +1 & 1.000 & -2 & 0.000 & +3 \\
\hline torsion & 349 & 6 & 9 & 6 & 0.000 & +1 & 1.000 & -2 & 0.000 & +3 \\
\hline torsion & 350 & 6 & 9 & 6 & 0.000 & +1 & 1.000 & -2 & 0.000 & +3 \\
\hline torsion & 351 & 6 & 9 & 6 & 0.000 & +1 & 1.000 & -2 & 0.000 & +3 \\
\hline torsion & 352 & 6 & 9 & 6 & 0.000 & +1 & 1.000 & -2 & 0.000 & +3 \\
\hline torsion & 338 & 6 & 9 & 7 & 0.000 & +1 & 1.000 & -2 & 0.000 & +3 \\
\hline torsion & 339 & 6 & 9 & 7 & 0.000 & +1 & 1.000 & -2 & 0.000 & +3 \\
\hline torsion & 340 & 6 & 9 & 7 & 0.000 & +1 & 1.000 & -2 & 0.000 & +3 \\
\hline torsion & 341 & 6 & 9 & 7 & 0.000 & +1 & 1.000 & -2 & 0.000 & +3 \\
\hline torsion & 343 & 6 & 9 & 7 & 0.000 & +1 & 1.000 & -2 & 0.000 & +3 \\
\hline torsion & 344 & 6 & 9 & 7 & 0.000 & +1 & 1.000 & -2 & 0.000 & +3 \\
\hline torsion & 345 & 6 & 9 & 7 & 0.000 & +1 & 1.000 & -2 & 0.000 & +3 \\
\hline torsion & 346 & 6 & 9 & 7 & 0.000 & +1 & 1.000 & -2 & 0.000 & +3 \\
\hline torsion & 347 & 6 & 9 & 7 & 0.000 & +1 & 1.000 & -2 & 0.000 & +3 \\
\hline torsion & 348 & 6 & 9 & 7 & 0.000 & +1 & 1.000 & -2 & 0.000 & +3 \\
\hline torsion & 349 & 6 & 9 & 7 & 0.000 & +1 & 1.000 & -2 & 0.000 & +3 \\
\hline torsion & 350 & 6 & 9 & 7 & 0.000 & +1 & 1.000 & -2 & 0.000 & +3 \\
\hline torsion & 351 & 6 & 9 & 7 & 0.000 & +1 & 1.000 & -2 & 0.000 & +3 \\
\hline torsion & 352 & 6 & 9 & 7 & 0.000 & +1 & 1.000 & -2 & 0.000 & +3 \\
\hline
\end{tabular}




\section{(3) Basis for MM3 parameterization}

Parameters for phosphine oxides. Phosphine oxide parameters were taken from a recent publication De Silva, N.; Federico, Z.; Hay, B. P.; Gordon, M. S.; and Windus, T. L. "Conformations of Phosphine Oxides" J. Phys. Chem. A 2015, 119, 8765-8773. Following the methodology reported therein, MM3 torsion parameters were further refined by fitting to MP2/cc-pVTZ potential energy surfaces for 18 additional bond rotations in selected phosphine oxide molecules. The potential surfaces and fits are given below, where black E(ref) is the MP2/cc-pVTZ surface and red E(calc) is the surface calculated with the modified MM3 parameters given above.
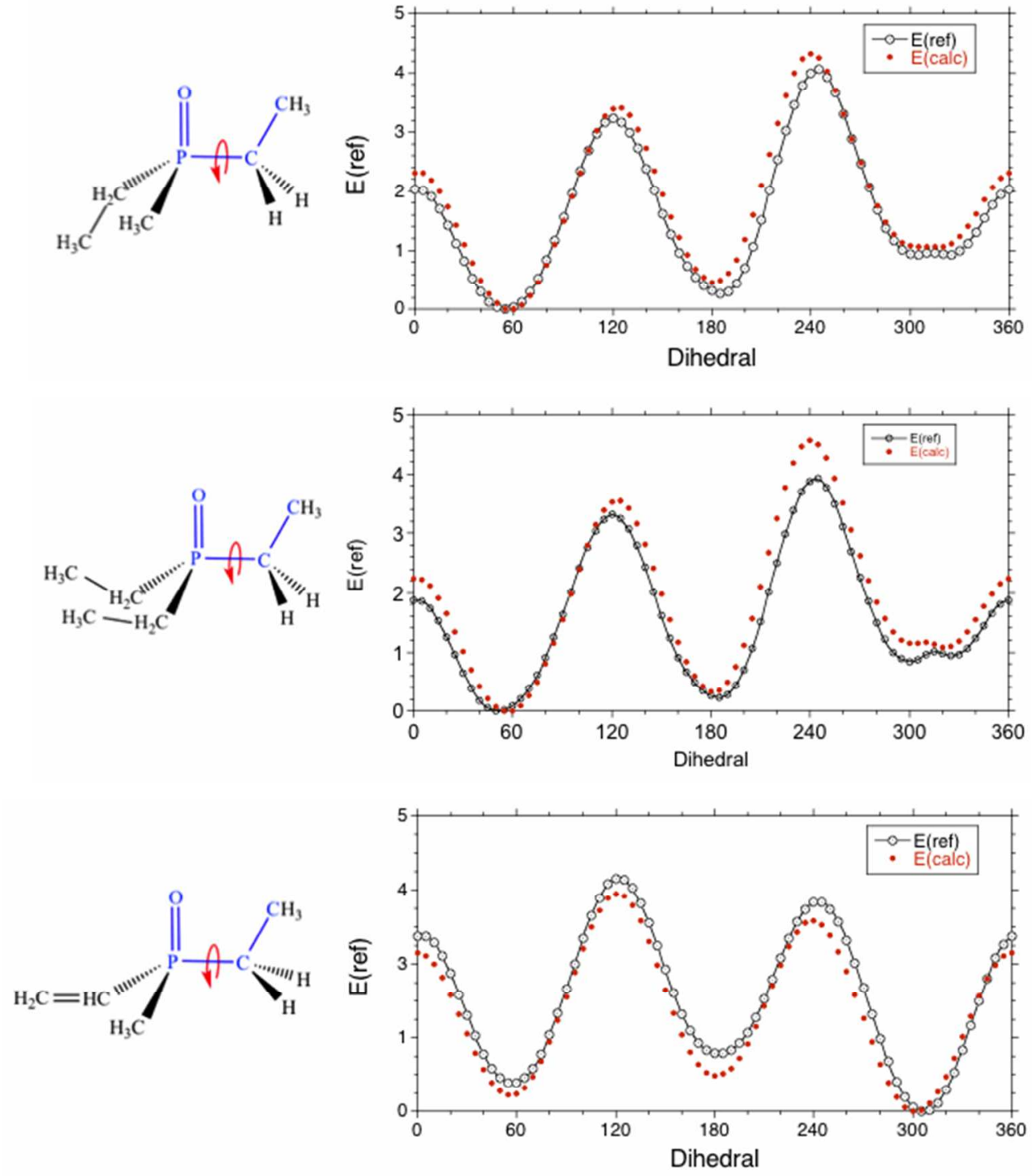

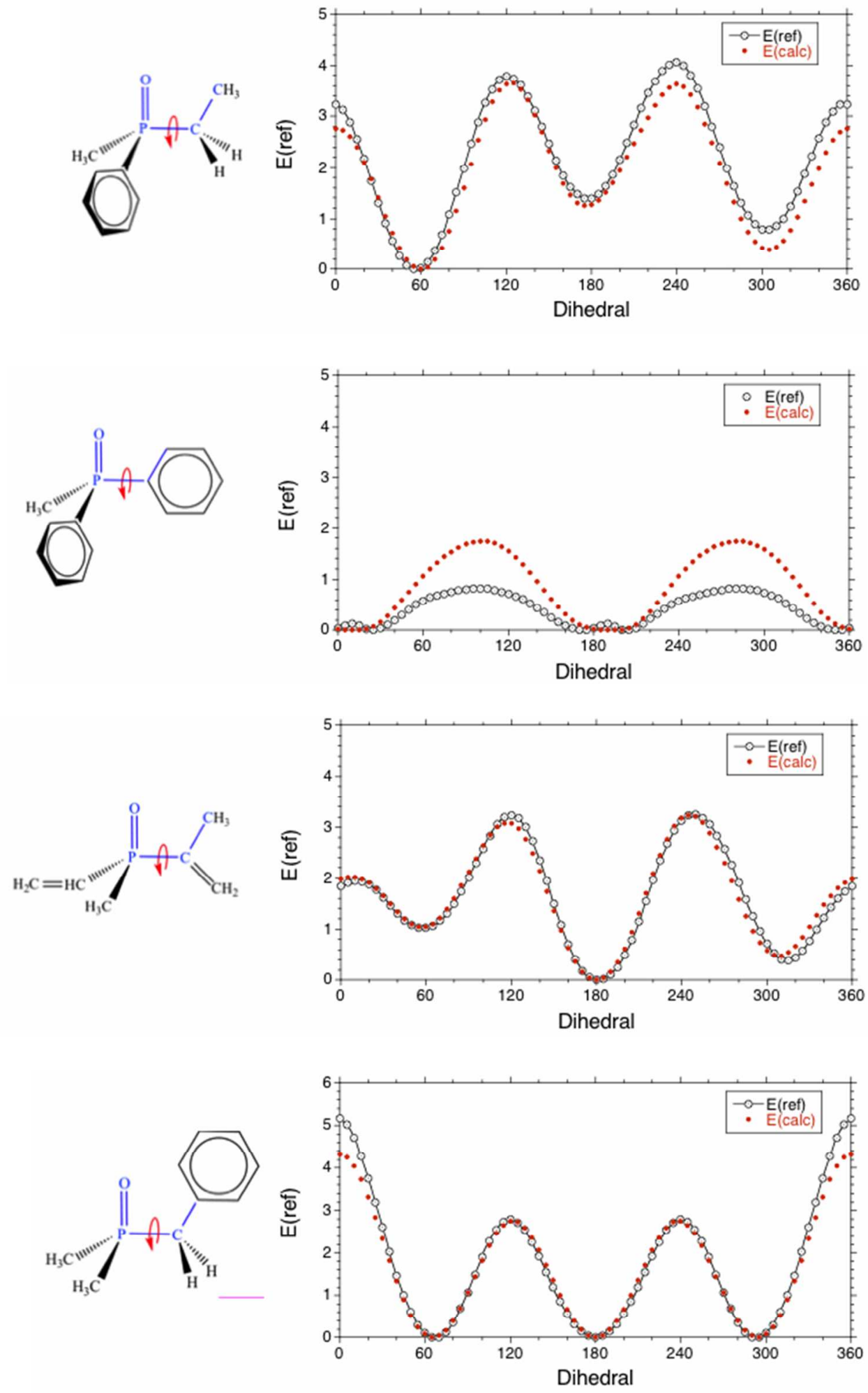

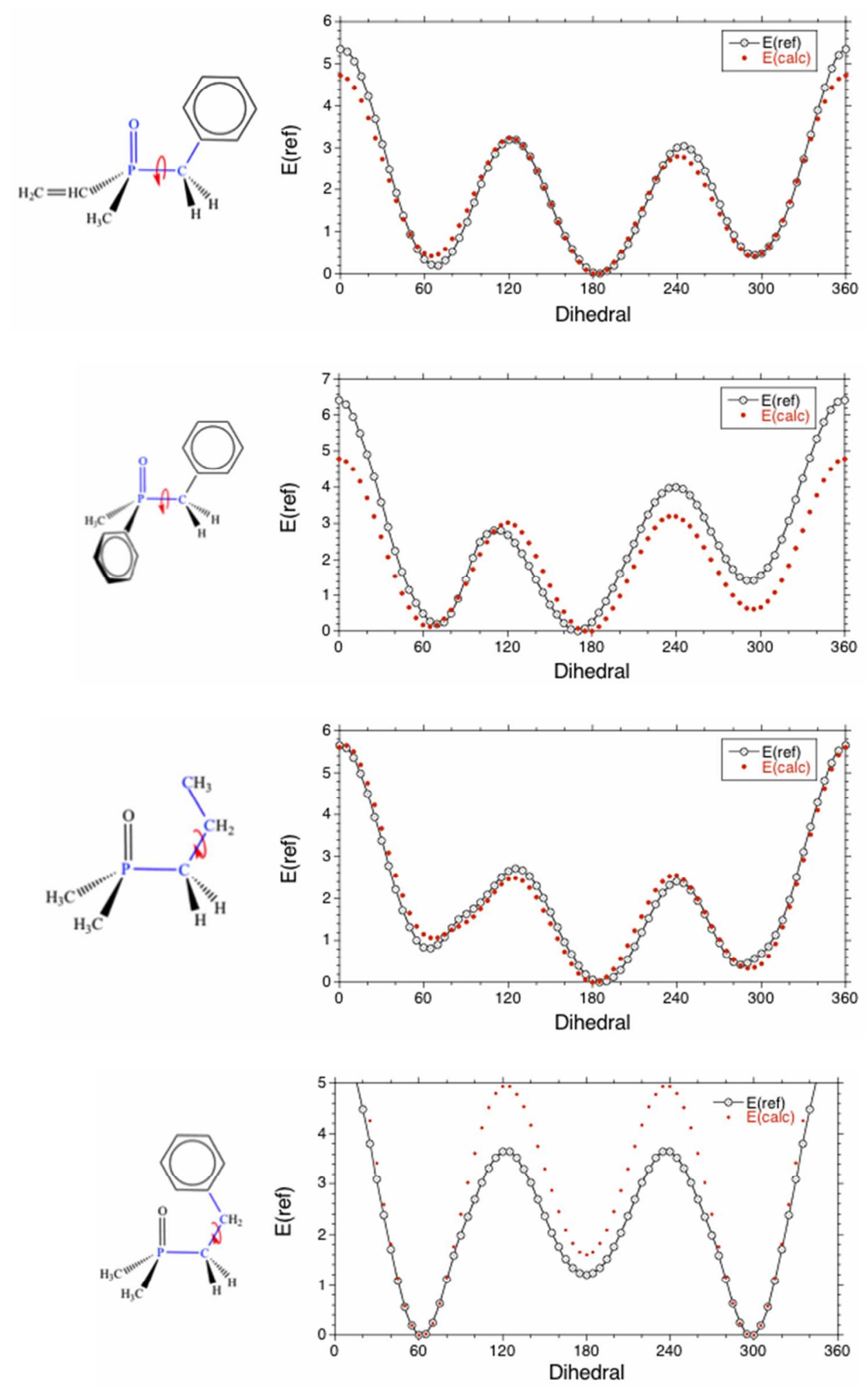

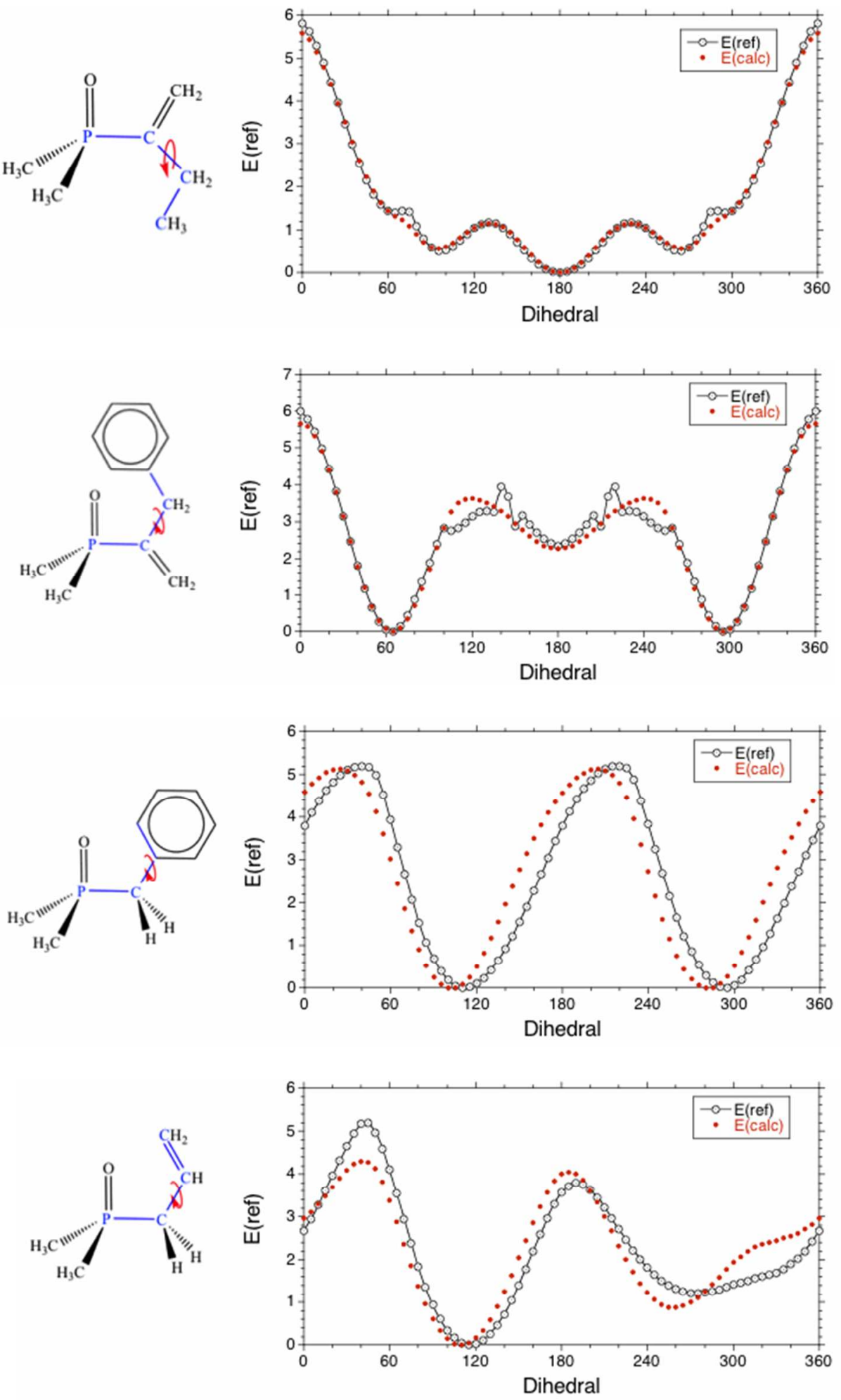

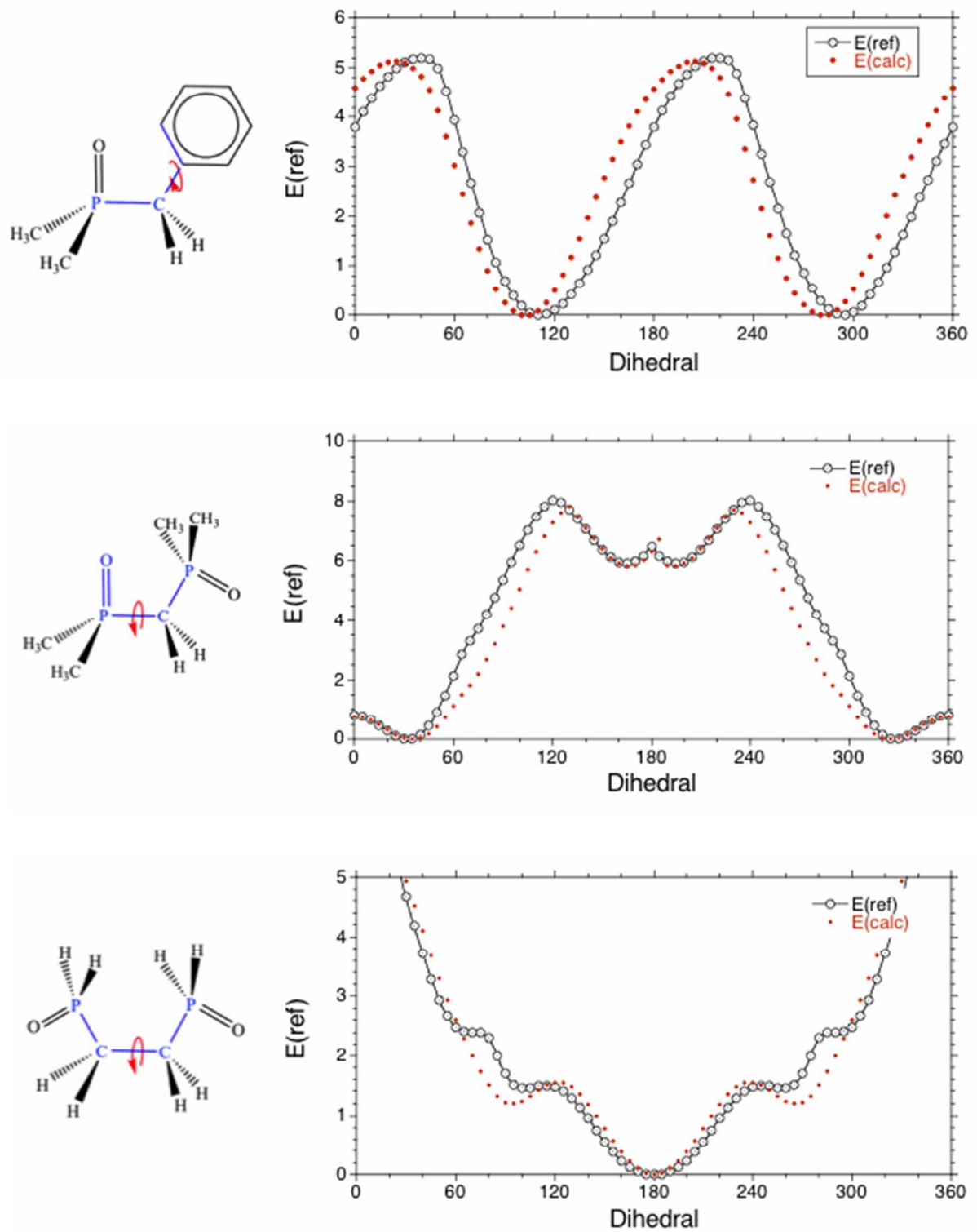

Although it is not possible to reproduce exactly several of the current MP2 rotational PES by adjustment of $V_{1}, V_{2}$, and $V_{3}$ terms alone, the MM3 model predicts correctly the location and relative energy of the rotamers, in other words, the model correctly predicts the shape of phosphine oxide molecules and is expected to provide a reasonable estimate of relative conformational energies for this class of molecules. 
Metal-dependent MM3 parameters. Following the development and validation of MM3 parameters for phosphine oxide molecules, the next step was to extend this model to allow the treatment of lanthanide complexes with these ligands. This was done using the PCModel software implementation of MM3, which contains the ability to define a lanthanide atom as a points-on-a-sphere (POS) center to which all other ligands are formally connected with a bond [see Hay, B. P. "The Application of Molecular Mechanics in Coordination Chemistry" Coord. Chem. Rev. 1993, 126, 177236]. To extend an organic force field parameter set to handle metal complexes, the POS approach requires the addition of M-L stretching, M-L-X bending, and M-L-X$\mathrm{X}$ torsion parameters, where $\mathrm{M}$ is the metal, $\mathrm{L}$ is a donor atom, and $\mathrm{X}$ is any other atom. Thus, it was necessary to add parameters for the $\mathrm{M}-\mathrm{O}(\mathrm{P})$ bond, $\mathrm{M}-\mathrm{O}-\mathrm{P}$ angle, and $\mathrm{M}-\mathrm{O}=\mathrm{P}-\mathrm{C}$ torsions. With a POS metal center, there are no explicitly declared L$\mathrm{M}-\mathrm{L}$ angles or $\mathrm{L}-\mathrm{M}-\mathrm{L}-\mathrm{X}$ dihedral angles. The angles subtended at the metal and torsion angles about $\mathrm{M}-\mathrm{L}$ bonds are dictated by steric repulsion between the set of $\mathrm{L}$ atoms attached to the metal.

It is possible to derive a set of parameters based on experimentally observed structural features in x-ray structure data. A search of the CSD without any filters other than 'no powder structures' yielded a total of 424 examples of a phosphine oxide coordinated with a lanthanide through a $\mathrm{Ln}-\mathrm{O}$ bond not in a ring. In other words, these are examples where the geometries associated with the lanthanide-phosphine oxide interaction are not contaminated by geometric constraints that are present when the phosphine oxide is in a chelate ring. Geometric features for phosphine oxide - lanthanide interaction were collected from these data.

Where sufficient data were available (more than 10 examples), the $\mathrm{M}-\mathrm{O}(\mathrm{P})$ distance was averaged for each lanthanide. A plot of the observed average $\mathrm{M}-\mathrm{O}(\mathrm{P})$ distance versus Shannon's ionic radius [Shannon, R. D. Acta Cryst. 1976, A32, 751-767] for coordination number 8, shown in Figure S1, reveals the expected linear correlation. Average distances range from a low of $2.18 \AA$ for Lu to a high of $2.42 \AA$ for La.

This data was used to generate a set of stretching parameters for the $\mathrm{M}-\mathrm{O}(\mathrm{P})$ interaction, in other words, the ideal distance $\mathrm{r}_{0}$ and the stretching force constant $\mathrm{k}_{\mathrm{r}}$. The 
best-fit line through the data in Figure $\mathrm{S} 1$ was used to compute an initial set of $\mathrm{r}_{0}$ values for all lanthanides. Using these distances, Badger's rule [Badger, R. M. J. Chem. Phys.

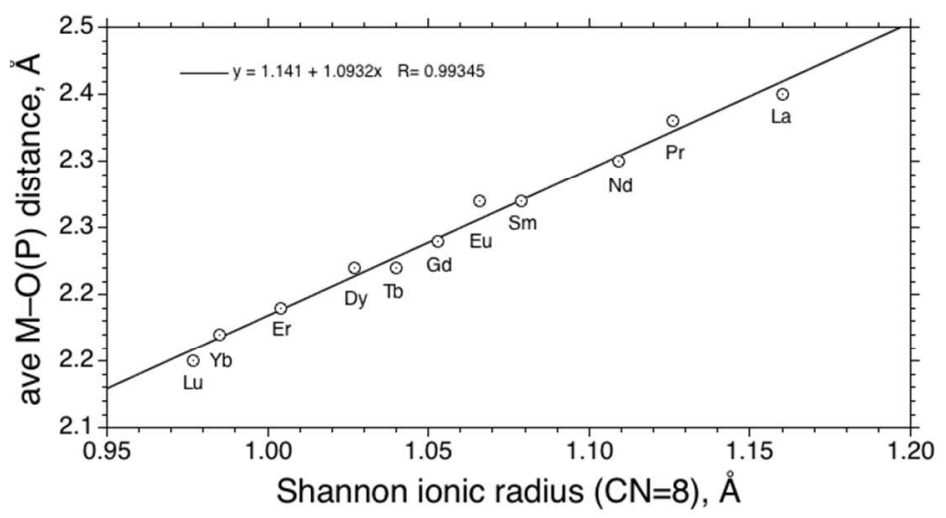

Figure S1. Plot of average $\mathrm{M}-\mathrm{O}(\mathrm{P})$ distance versus Shannon ionic radius $(\mathrm{CN}=8)$

1934, 2, 128-131; J. Chem. Phys. 1935, 3, 710-714] was used to compute stretching force constants:

$$
\mathrm{k}_{\mathrm{r}}(\operatorname{mdyn} / \AA)=10 \times[0.49 /(\mathrm{M}-\mathrm{O} \text { distance }-1.26)]^{3}
$$

This yielded $\mathrm{k}_{\mathrm{r}}$ values ranging from $0.775 \mathrm{mdyn} / \AA$ for $\mathrm{La}$ to $1.376 \mathrm{mydn} / \AA$ for $\mathrm{Lu}$. Subsequently, the $r_{0}$ values were adjusted slightly to give the best overall agreement between observed and calculated $\mathrm{M}-\mathrm{O}$ distances in geometry optimized metal complexes taken from the Cambridge Database.

A histogram showing the distribution of $\mathrm{M}-\mathrm{O}=\mathrm{P}$ angles is shown in Figure $\mathrm{S} 2$. The values range from 140 to $180^{\circ}$, with a maximum at $167^{\circ}$. This data was used to assign parameters for the $\mathrm{M}-\mathrm{O}=\mathrm{P}$ bending interaction, the ideal angle $\theta_{0}$ and the bending force constant $\mathrm{k}_{\theta}$. The $\theta_{0}$ parameter was assigned a value of $167^{\circ}$ for all lanthanides. 


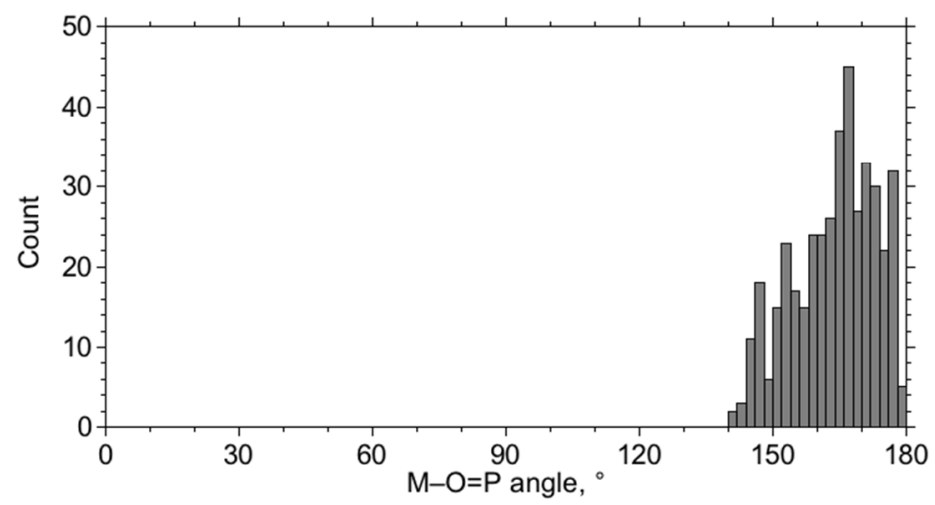

Figure S2. Histogram showing the distribution of 424 observations of the $M-O=P$ angle for phosphine oxides not in chelate rings.

The following procedure was used to estimate an effective average value of $\mathrm{k}_{\theta}$. First, by variation of $\mathrm{k}_{\theta}$, a series of theoretical bending potential energy surfaces were calculated using the MM3 bending potential function:

$$
\begin{array}{r}
\mathrm{E}(\mathrm{kcal} / \mathrm{mol})=0.2191 \times \mathrm{k}_{\theta} \times \Delta \theta^{2} \times\left[1+\mathrm{CFx} \Delta \theta+\mathrm{QFx} \Delta \theta^{2}+\mathrm{PFx} \Delta \theta^{3}+\mathrm{SFx} \Delta \theta^{4}\right] \\
\mathrm{CF}=-1400 \times 10^{-5} ; \mathrm{QF}=5.6 \times 10^{-5} ; \mathrm{PF}=-0.07 \times 10^{-5} ; \mathrm{SF}=0.0022 \times 10^{-5}
\end{array}
$$

These potential energy surfaces, shown in Figure S3, were used to compute the expected distribution of 424 data points over the range of 140 to $180^{\circ}$. The calculated distributions are compared to the observed distribution in Figure S4. It can be seen that the observed distribution is best reproduced when $\mathrm{k}_{\theta}$ lies between 0.1 and 0.2 mdyn- $\AA / \operatorname{rad}^{2}$. Thus, $\mathrm{k}_{\theta}$ was assigned an average value of 0.15 mdyn$\AA / \mathrm{rad}^{2}$. The actual value for this bending force constant should be metal-dependent, increasing on going from La(III) to Lu(III). Assigning the average value to Gd(III) and assuming a dependence with the same percent change as that observed in the stretching force constants, yielded a final set of bending force constants that range

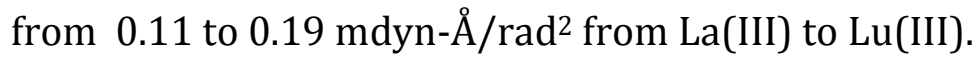




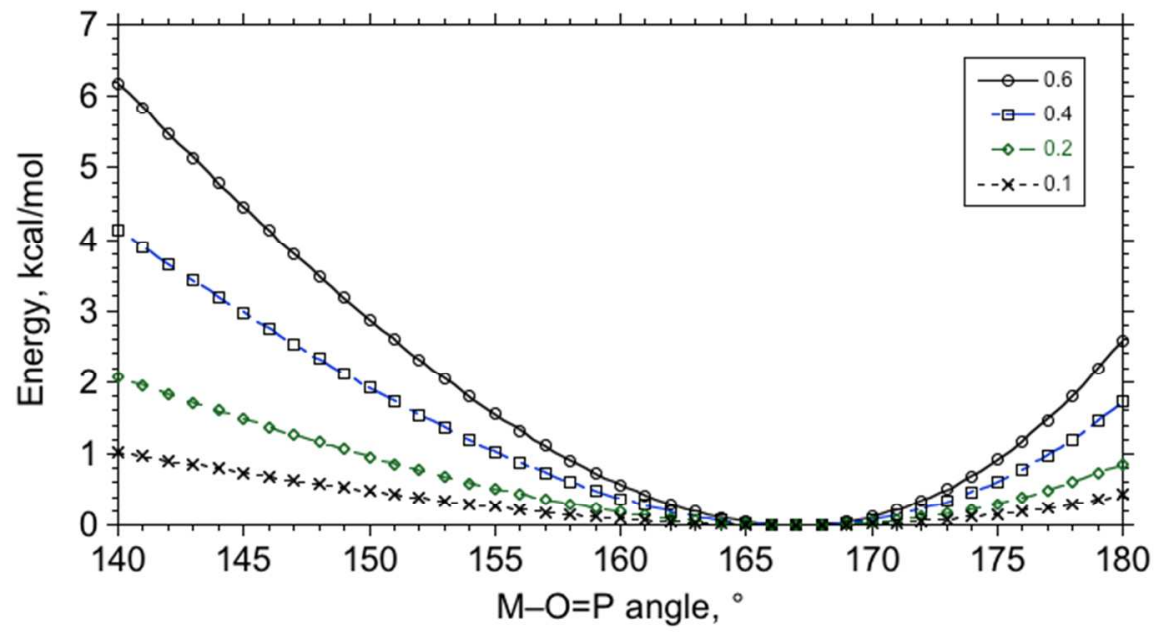

Figure S3. Computed PES for $\mathrm{M}-\mathrm{O}=\mathrm{P}$ angle distortion as a function of $\mathrm{k}_{\theta}$ (value in legend).
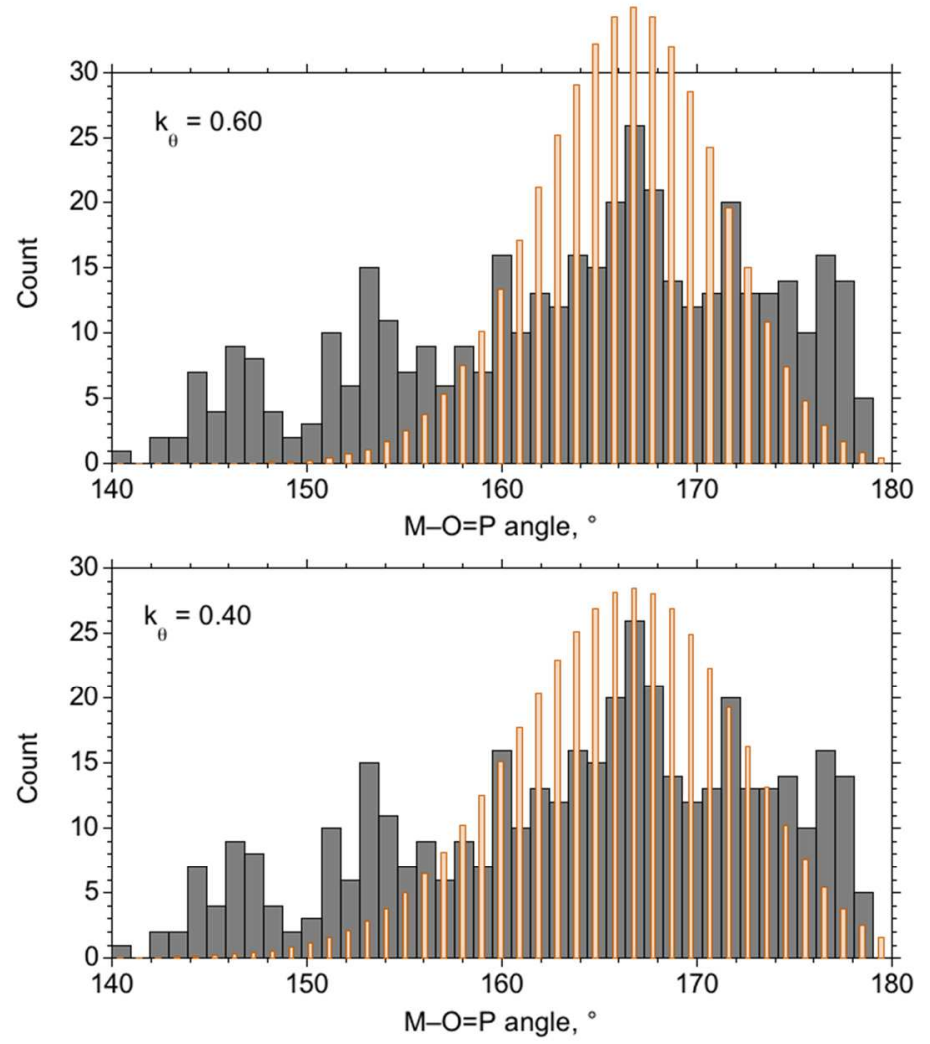

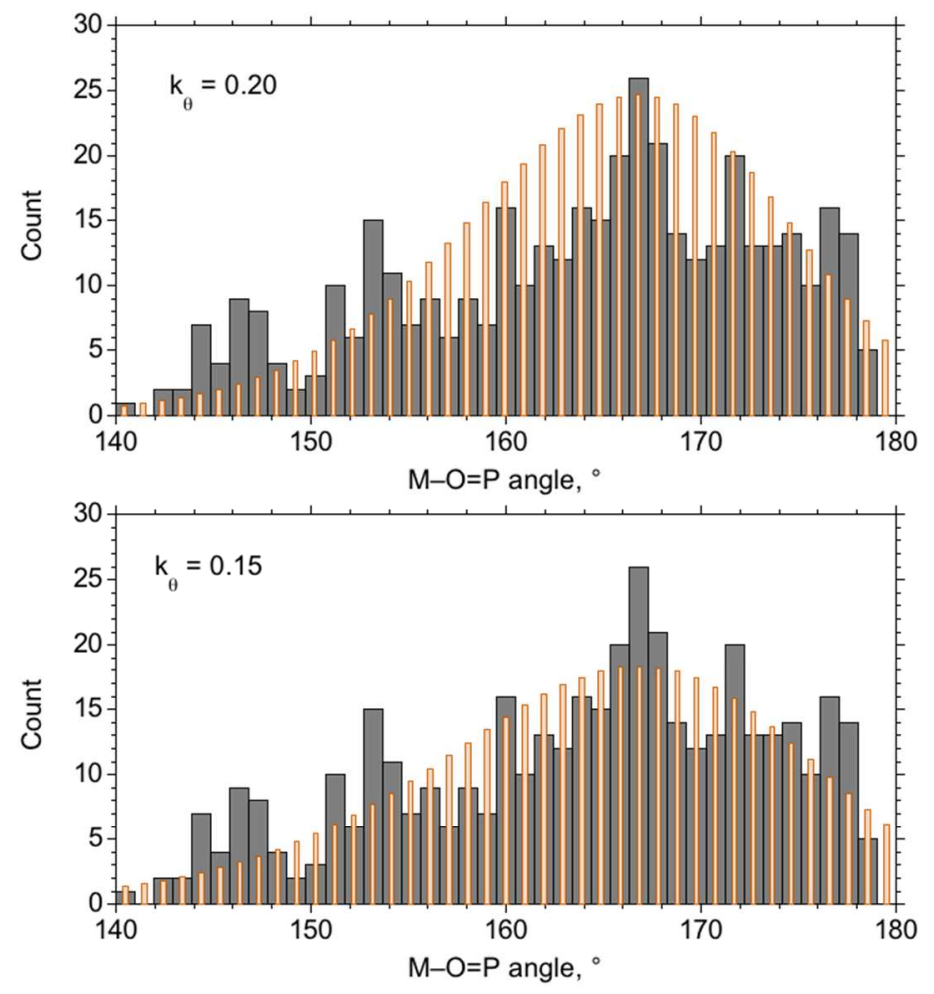

Figure S4. Obs (grey) vs. calc (orange) distributions at different values of $\mathrm{k}_{\theta}$.

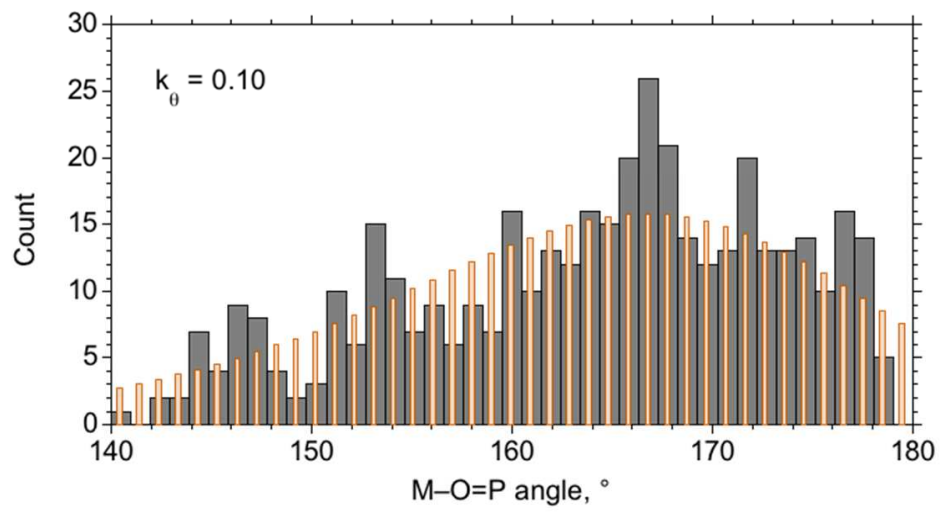

Figure S4 (cont.) Obs (grey) vs. calc (orange) distributions at different values of $\mathrm{k}_{\theta}$.

A histogram showing the distribution of all $\mathrm{M}-\mathrm{O}-\mathrm{P}-\mathrm{C}$ angles is shown in Figure S5. The values are randomly distributed over $0-360^{\circ}$ indicating no apparent preference. This is not surprising given the near linearity of the M-O-P angle and the relatively long, $\geq 2 \AA, M-0$ distances when compared to the distances encountered in organic molecules. When separate distributions are plotted for $\mathrm{M}-$ $\mathrm{O}-\mathrm{P}-\mathrm{C}($ aliphatic) and $\mathrm{M}-\mathrm{O}-\mathrm{P}-\mathrm{C}($ phenyl), not shown, there is no evidence for any 
preference with either group. Thus, $\mathrm{M}-\mathrm{O}-\mathrm{P}-\mathrm{C}$ dihedral parameters, $\mathrm{V}_{1}, \mathrm{~V}_{2}$, and $\mathrm{V}_{3}$, were set to zero.

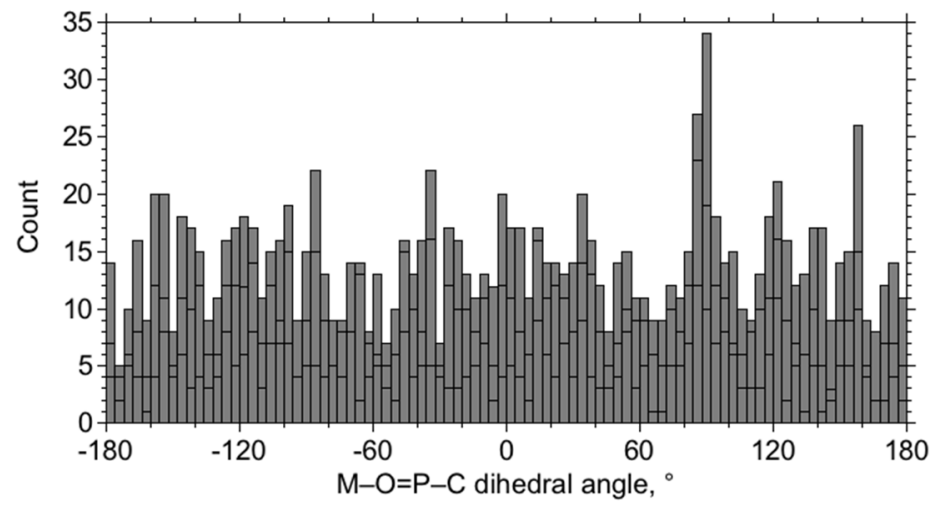

Figure S5. Histogram showing the distribution of all $\mathrm{Ln}-\mathrm{O}=\mathrm{P}-\mathrm{C}$ angles in $\mathrm{x}$-ray structures.

A good test of the validity of the model is to determine how well it reproduces the geometry of complexes in observed crystal structures. Because most of the crystal structures for lanthanide - phosphine oxide complexes contain other types of ligands bound to the lanthanide, it is necessary to create additional parameters to handle these other ligands. This becomes problematic if one attempts to model a wide range of auxiliary ligands and thus we restrict ourselves to two of the most common auxiliary ligands, nitrate and chloride. Stretching parameters for M$\mathrm{O}$ (nitrate) and $\mathrm{M}-\mathrm{Cl}$ were obtained in the same manner as described above, by fitting a line through a plot of observed average distances versus Shannon's ionic radii, shown in Figure S6, and through the use of Badger's rule. Additional bending and torsion parameters needed for nitrate were taken from prior work [Hay, B. P. "An Extension of Molecular Mechanics to High Coordinate Metal Complexes: Calculation of Structure in Aquo and Nitrato complexes of Lanthanide(III) Metal Ions" Inorg. Chem. 1991, 30, 2876-2884]. 


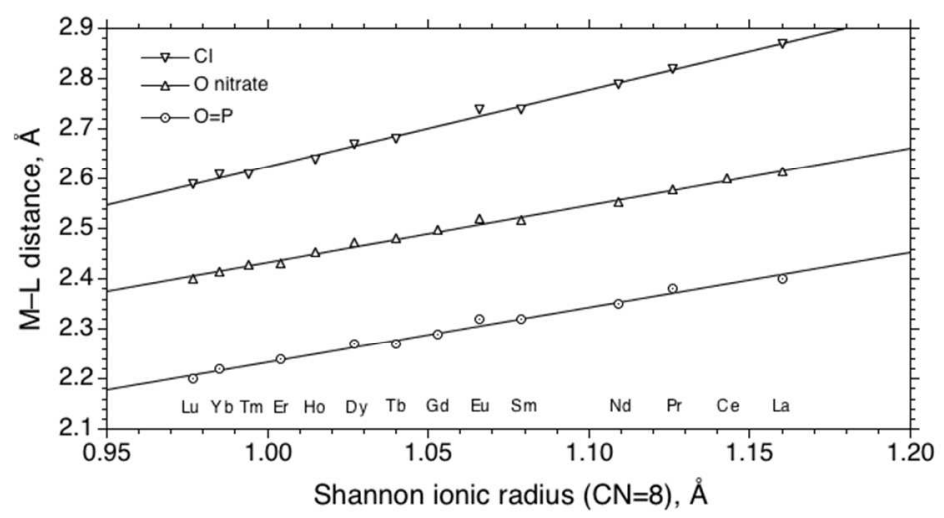

Figure S6. Plots of average observed M-L distances for phosphine oxide, bidentate nitrate, and chloride ligands versus Shannon ionic radius for coordination number 8.

The resulting extended MM3 model (see above for complete parameter set) was tested for its ability to reproduce known crystal structures. To perform this test, $\mathrm{x}$ ray crystal structure coordinates were used to prepare an input file for the calculation. The structure was then optimized with the MM3 force field as implemented in PCModel. The optimized coordinates were superimposed on the $\mathrm{x}$ ray coordinates and the mean absolute distance between every heavy (nonhydrogen) atom pair provided a measure of how well a crystal structure is reproduced. A representative superposition is shown below: 


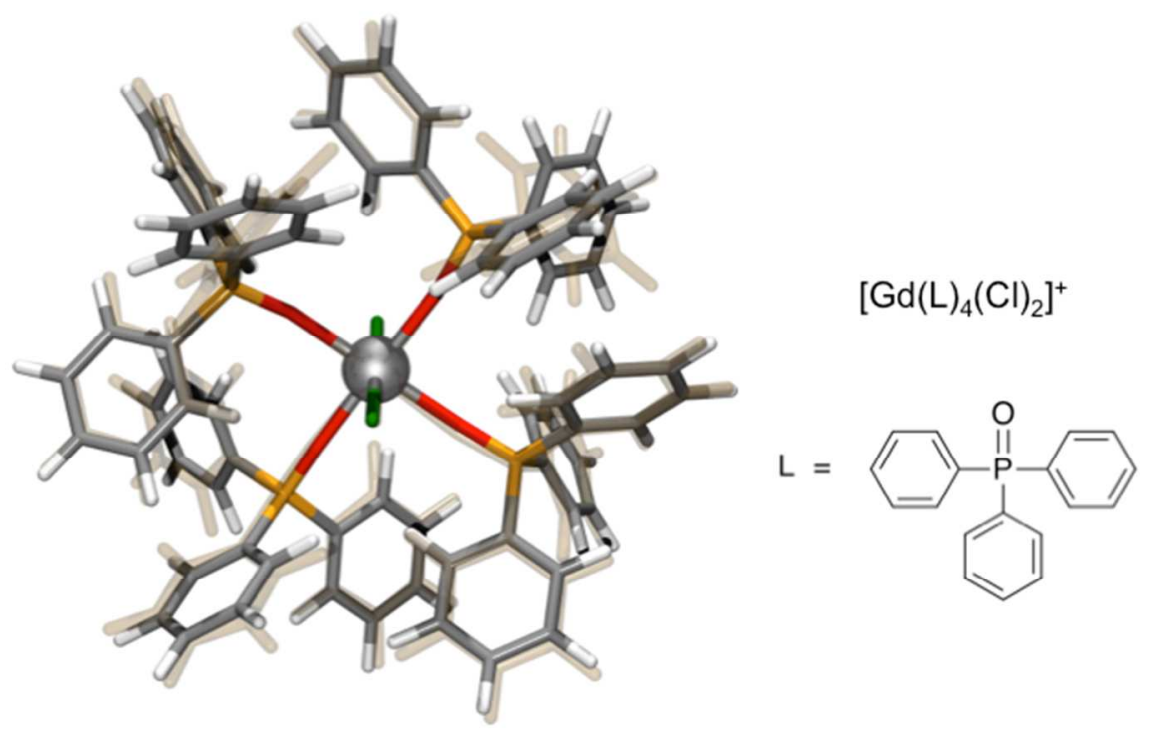

GEXRIK - Gd, overlay $0.352 \AA$

$\mathrm{Gd}-\mathrm{O}=\mathrm{P}$ calc dist $2.283 \mathrm{~A}(\Delta=-0.003 \AA)$

$\mathrm{Gd}-\mathrm{Cl}$ calc dist $2.696 \mathrm{~A}(\Delta=+0.041 \AA)$

additional examples of this stoichiometry:

EVEVOQ - Yb, overlay $0.336 \mathrm{~A}$

$\mathrm{Yb}-\mathrm{O}=\mathrm{P}$ calc dist $2.206 \mathrm{~A}(\Delta=-0.001 \AA)$

$\mathrm{Yb}-\mathrm{Cl}$ calc dist $2.592 \mathrm{~A}(\Delta=+0.004 \mathrm{~A})$

EVEVIK - Tb, overlay $0.358 \AA$

$\mathrm{Tb}-\mathrm{O}=\mathrm{P}$ calc dist $2.269 \AA(\Delta=+0.007 \mathrm{~A})$

The mean absolute deviations for 31 superpositions, which range from 0.21 to $0.55 \AA$, are typical for this type of complex. Where larger deviations are observed, they commonly arise from relatively small, $20-30^{\circ}$, rotations in the $\mathrm{M}-\mathrm{O}-\mathrm{P}-\mathrm{C}$ and/or $\mathrm{O}-\mathrm{P}-\mathrm{C}-\mathrm{C}$ dihedral angles that result in large deviations in the perimeter of the structure. The latter is common in ligands bearing phenyl substituents. Given the low barrier to phenyl rotation when two or more phenyl groups are present, such rotations are not unexpected on going from the crystalline environment where the complex is packed closely and interacting with other molecules to the gas phase. 


\section{(4) List of diphosphoranedione architectures}

The monocyclic and bicyclic frameworks below illustrate every possible way to distribute two $\mathrm{P}$ atoms within these ring systems such that they are separated by at least one $\mathrm{C}$ atom. Each $\mathrm{P}$ atom was substituted with an oxo group and, where needed, with a Me substituent to give a total coordination number of 4 . With bicylic frameworks, both cis and trans ring connections were considered. In each case, the stereochemistry yielding the most complementary Gd(III) complex was retained. Thus, a total of 65 candidate architectures were evaluated.
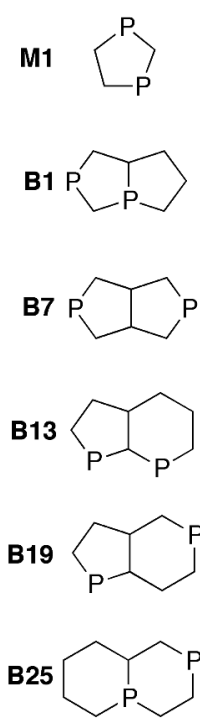
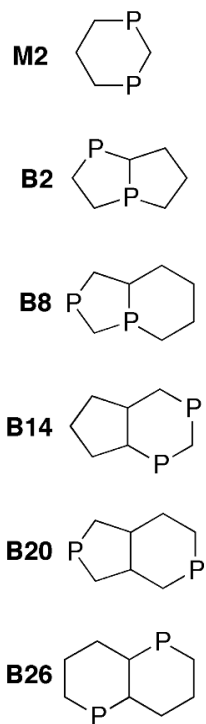
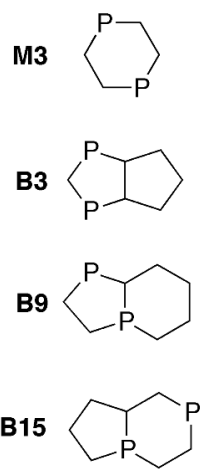<smiles>O=P(=O)PC1CC2CCCCP2C1</smiles><smiles>CC1CCPCC1PCCC(Br)Br</smiles>
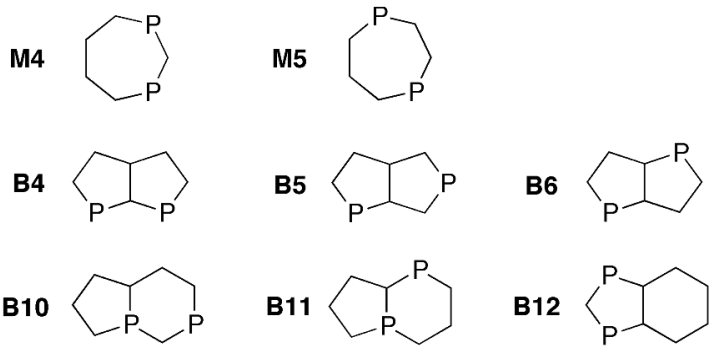

B16
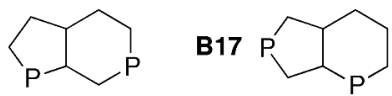

B18
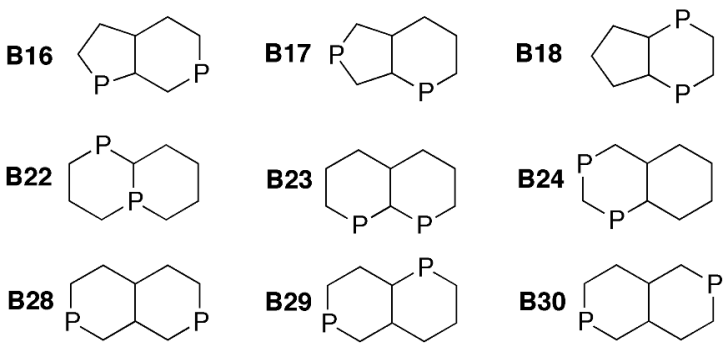
(5) Atomic coordinates for MM3 optimized metal complex and global minimum geometries for candidates 1-65 shown in Figures 7, 8, and 9 of the manuscript.

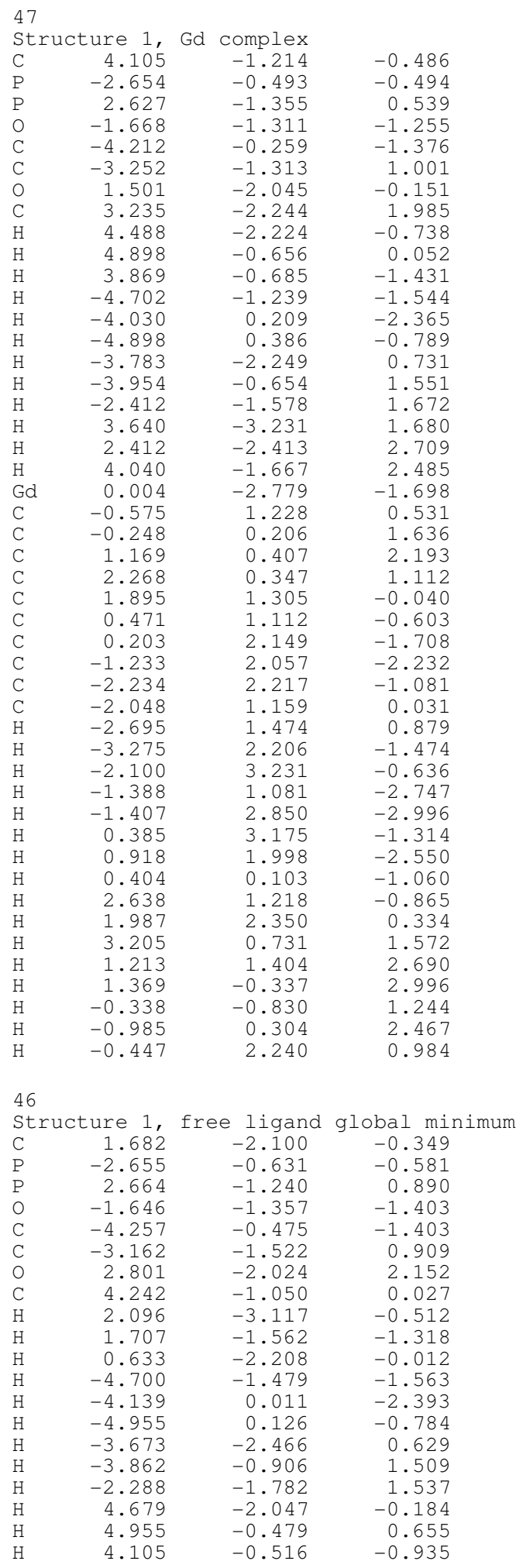




\begin{tabular}{|c|c|c|c|}
\hline $\mathrm{C}$ & -0.713 & 1.225 & 0.478 \\
\hline $\mathrm{C}$ & -0.386 & 0.284 & 1.650 \\
\hline $\mathrm{C}$ & 0.997 & 0.586 & 2.239 \\
\hline $\mathrm{C}$ & 2.139 & 0.487 & 1.204 \\
\hline $\mathrm{C}$ & 1.778 & 1.318 & -0.047 \\
\hline $\mathrm{C}$ & 0.364 & 1.081 & -0.622 \\
\hline $\mathrm{C}$ & 0.100 & 2.074 & -1.770 \\
\hline $\mathrm{C}$ & $-1 \cdot 322$ & 1.941 & $-2 \cdot 328$ \\
\hline $\mathrm{C}$ & -2.360 & 2.087 & -1.207 \\
\hline $\mathrm{C}$ & -2.163 & 1.064 & -0.064 \\
\hline $\mathrm{H}$ & -2.854 & 1.360 & 0.754 \\
\hline $\mathrm{H}$ & -3.388 & 2.024 & -1.628 \\
\hline $\mathrm{H}$ & -2.280 & 3.116 & -0.784 \\
\hline $\mathrm{H}$ & -1.439 & 0.958 & -2.837 \\
\hline $\mathrm{H}$ & -1.495 & 2.721 & -3.105 \\
\hline $\mathrm{H}$ & 0.253 & 3.117 & -1.408 \\
\hline $\mathrm{H}$ & 0.838 & 1.910 & -2.590 \\
\hline $\mathrm{H}$ & 0.317 & 0.055 & -1.038 \\
\hline $\mathrm{H}$ & 2.537 & 1.157 & -0.846 \\
\hline $\mathrm{H}$ & 1.860 & 2.395 & 0.227 \\
\hline $\mathrm{H}$ & 3.020 & 0.982 & 1.668 \\
\hline $\mathrm{H}$ & 0.983 & 1.619 & 2.658 \\
\hline $\mathrm{H}$ & 1.196 & -0.086 & 3.105 \\
\hline $\mathrm{H}$ & -0.408 & -0.774 & 1.315 \\
\hline $\mathrm{H}$ & -1.158 & 0.391 & 2.447 \\
\hline $\mathrm{H}$ & -0.637 & 2.266 & 0.873 \\
\hline \multicolumn{4}{|c|}{38} \\
\hline \multicolumn{4}{|c|}{ Structure 2, Gd con } \\
\hline $\mathrm{P}$ & -2.241 & -0.633 & 0.828 \\
\hline O & -1.261 & -1.742 & 0.991 \\
\hline $\mathrm{C}$ & -3.663 & -1.099 & -0.178 \\
\hline $\mathrm{C}$ & -2.962 & -0.058 & 2.374 \\
\hline $\mathrm{P}$ & 2.306 & -0.588 & -0.665 \\
\hline O & 1.449 & -1.797 & -0.525 \\
\hline $\mathrm{C}$ & 3.756 & -0.631 & 0.407 \\
\hline $\mathrm{C}$ & 2.984 & -0.354 & -2.317 \\
\hline $\mathrm{Gd}$ & 0.181 & -3.406 & 0.446 \\
\hline $\mathrm{C}$ & 0.435 & 1.536 & -1.212 \\
\hline $\mathrm{C}$ & -0.140 & 2.670 & -0.344 \\
\hline $\mathrm{C}$ & -0.607 & 1.740 & 0.791 \\
\hline $\mathrm{C}$ & 0.716 & 0.985 & 1.118 \\
\hline $\mathrm{C}$ & 1.502 & 0.983 & -0.234 \\
\hline $\mathrm{C}$ & -0.797 & 0.589 & $-1 \cdot 317$ \\
\hline $\mathrm{C}$ & -1.597 & 0.850 & 0.000 \\
\hline $\mathrm{H}$ & -4.187 & -1.961 & 0.282 \\
\hline $\mathrm{H}$ & -4.374 & -0.252 & -0.262 \\
\hline $\mathrm{H}$ & -3.329 & -1.388 & -1.195 \\
\hline $\mathrm{H}$ & -3.506 & -0.887 & 2.871 \\
\hline $\mathrm{H}$ & -2.165 & 0.302 & 3.056 \\
\hline $\mathrm{H}$ & -3.672 & 0.773 & 2.184 \\
\hline $\mathrm{H}$ & 4.372 & -1.523 & 0.175 \\
\hline $\mathrm{H}$ & 4.374 & 0.278 & 0.262 \\
\hline $\mathrm{H}$ & 3.440 & -0.684 & 1.469 \\
\hline $\mathrm{H}$ & 3.617 & $-1 \cdot 222$ & -2.593 \\
\hline $\mathrm{H}$ & 2.162 & -0.265 & -3.056 \\
\hline $\mathrm{H}$ & 3.601 & 0.566 & -2.354 \\
\hline $\mathrm{H}$ & 0.843 & 1.869 & -2.194 \\
\hline $\mathrm{H}$ & 0.630 & 3.406 & -0.014 \\
\hline $\mathrm{H}$ & -0.976 & 3.215 & -0.840 \\
\hline $\mathrm{H}$ & -1.061 & 2.269 & 1.661 \\
\hline $\mathrm{H}$ & 1.293 & 1.532 & 1.898 \\
\hline $\mathrm{H}$ & 0.522 & -0.038 & 1.507 \\
\hline $\mathrm{H}$ & 2.303 & 1.750 & -0.164 \\
\hline $\mathrm{H}$ & $-1 \cdot 418$ & 0.855 & -2.203 \\
\hline $\mathrm{H}$ & -0.493 & -0.474 & -1.435 \\
\hline $\mathrm{H}$ & -2.473 & 1.487 & -0.253 \\
\hline \multicolumn{4}{|c|}{37} \\
\hline St & =ure 2, & free ligand & global minimum \\
\hline $\mathrm{P}$ & -2.291 & -0.702 & 0.556 \\
\hline 0 & $-1 \cdot 342$ & -1.830 & 0.767 \\
\hline $\mathrm{C}$ & -3.662 & -1.110 & -0.543 \\
\hline $\mathrm{C}$ & -3.099 & -0.144 & 2.068 \\
\hline $\mathrm{P}$ & 2.159 & -0.819 & -1.350 \\
\hline 0 & 2.466 & -0.676 & -2.802 \\
\hline $\mathrm{C}$ & 1. 221 & -2.310 & -0.975 \\
\hline $\mathrm{C}$ & 3.634 & -1.085 & -0.339 \\
\hline $\mathrm{C}$ & 0.413 & 1.462 & -1.453 \\
\hline $\mathrm{C}$ & 0.004 & 2.533 & -0.425 \\
\hline $\mathrm{C}$ & -0.463 & 1.520 & 0.636 \\
\hline
\end{tabular}




$\begin{array}{rrrr}\mathrm{C} & 0.811 & 0.632 & 0.775 \\ \mathrm{C} & 1.488 & 0.710 & -0.629 \\ \mathrm{C} & -0.902 & 0.640 & -1.564 \\ \mathrm{C} & -1.580 & 0.806 & -0.166 \\ \mathrm{H} & -4.240 & -1.961 & -0.129 \\ \mathrm{H} & -4.340 & -0.239 & -0.656 \\ \mathrm{H} & -3.276 & -1.395 & -1.543 \\ \mathrm{H} & -3.679 & -0.976 & 2.518 \\ \mathrm{H} & -2.340 & 0.196 & 2.802 \\ \mathrm{H} & -3.789 & 0.697 & 1.852 \\ \mathrm{H} & 1.765 & -3.197 & -1.359 \\ \mathrm{H} & 1.096 & -2.430 & 0.119 \\ \mathrm{H} & 0.226 & -2.285 & -1.460 \\ \mathrm{H} & 4.146 & -2.018 & -0.650 \\ \mathrm{H} & 4.340 & -0.238 & -0.462 \\ \mathrm{H} & 3.360 & -1.168 & 0.733 \\ \mathrm{H} & 0.783 & 1.870 & -2.423 \\ \mathrm{H} & 0.857 & 3.163 & -0.080 \\ \mathrm{H} & -0.814 & 3.197 & -0.791 \\ \mathrm{H} & -0.808 & 1.980 & 1.592 \\ \mathrm{H} & 1.490 & 1.051 & 1.553 \\ \mathrm{H} & 0.564 & -0.404 & 1.083 \\ \mathrm{H} & 2.349 & 1.408 & -0.539 \\ \mathrm{H} & -1.557 & 1.060 & -2.362 \\ \mathrm{H} & -0.704 & -0.420 & -1.826 \\ \mathrm{H} & -2.413 & 1.533 & -0.282\end{array}$

39

$\begin{array}{lrrr}\text { Structure 3, Gd } & \text { complex } & \\ \mathrm{P} & -2.404 & -0.914 & 0.236 \\ \mathrm{O} & -1.509 & -2.102 & 0.303 \\ \mathrm{C} & -3.552 & -0.975 & -1.155 \\ \mathrm{C} & -3.482 & -0.727 & 1.666 \\ \mathrm{P} & 2.396 & -0.879 & -0.348 \\ \mathrm{O} & 1.485 & -2.011 & -0.676 \\ \mathrm{C} & 3.541 & -1.263 & 0.992 \\ \mathrm{C} & 3.478 & -0.393 & -1.703 \\ \mathrm{Gd} & -0.022 & -3.677 & -0.369 \\ \mathrm{C} & 0.752 & 1.388 & -0.870 \\ \mathrm{C} & 1.550 & 0.632 & 0.224 \\ \mathrm{C} & 0.582 & 0.364 & 1.420 \\ \mathrm{C} & -0.733 & 1.160 & 1.246 \\ \mathrm{C} & -0.340 & 2.592 & 0.905 \\ \mathrm{C} & 0.377 & 2.714 & -0.219 \\ \mathrm{C} & -1.539 & 0.676 & 0.012 \\ \mathrm{C} & -0.572 & 0.668 & -1.215 \\ \mathrm{H} & -4.196 & -1.875 & -1.075 \\ \mathrm{H} & -4.198 & -0.074 & -1.165 \\ \mathrm{H} & -2.991 & -1.026 & -2.110 \\ \mathrm{H} & -4.165 & -1.598 & 1.743 \\ \mathrm{H} & -2.880 & -0.670 & 2.595 \\ \mathrm{H} & -4.090 & 0.197 & 1.575 \\ \mathrm{H} & 4.173 & -2.131 & 0.713 \\ \mathrm{H} & 4.198 & -0.395 & 1.202 \\ \mathrm{H} & 2.977 & -1.517 & 1.913 \\ \mathrm{H} & 4.149 & -1.234 & -1.972 \\ \mathrm{H} & 2.878 & -0.123 & -2.595 \\ \mathrm{H} & 4.097 & 0.479 & -1.409 \\ \mathrm{H} & 1.368 & 1.570 & -1.781 \\ \mathrm{H} & 2.345 & 1.324 & 0.580 \\ \mathrm{H} & 1.078 & 0.663 & 2.373 \\ \mathrm{H} & 0.349 & -0.719 & 1.521 \\ \mathrm{H} & -1.347 & 1.142 & 2.176 \\ \mathrm{H} & -0.673 & 3.445 & 1.521 \\ \mathrm{H} & 0.722 & 3.677 & -0.630 \\ \mathrm{H} & -2.324 & 1.440 & -0.180 \\ \mathrm{H} & -1.063 & 1.178 & -2.076 \\ \mathrm{H} & -0.353 & -0.368 & -1.554\end{array}$

$\begin{array}{lrcc}38 & & \\ \text { Structure 3, } & \text { free ligand } & \text { global minimum } \\ \text { P } & -2.375 & -1.297 & 0.126 \\ \mathrm{O} & -1.415 & -2.386 & 0.458 \\ \mathrm{C} & -3.407 & -1.651 & -1.311 \\ \mathrm{C} & -3.580 & -0.973 & 1.428 \\ \mathrm{P} & 2.428 & -1.011 & -0.801 \\ \mathrm{O} & 3.097 & -0.745 & -2.107 \\ \mathrm{C} & 1.509 & -2.560 & -0.798\end{array}$




$\begin{array}{rrrr}\mathrm{C} & 3.601 & -1.286 & 0.546 \\ \mathrm{H} & -4.013 & -2.561 & -1.128 \\ \mathrm{H} & -2.774 & -1.822 & -2.205 \\ \mathrm{H} & -4.090 & -0.802 & -1.515 \\ \mathrm{H} & -4.199 & -1.876 & 1.605 \\ \mathrm{H} & -4.247 & -0.135 & 1.139 \\ \mathrm{H} & -3.063 & -0.710 & 2.373 \\ \mathrm{H} & 2.178 & -3.389 & -1.104 \\ \mathrm{H} & 0.665 & -2.519 & -1.513 \\ \mathrm{H} & 1.123 & -2.788 & 0.215 \\ \mathrm{H} & 4.247 & -2.158 & 0.316 \\ \mathrm{H} & 3.064 & -1.482 & 1.497 \\ \mathrm{H} & 4.246 & -0.393 & 0.678 \\ \mathrm{C} & 0.586 & 1.097 & -1.287 \\ \mathrm{C} & 1.476 & 0.429 & -0.206 \\ \mathrm{C} & 0.597 & 0.184 & 1.058 \\ \mathrm{C} & -0.767 & 0.905 & 0.920 \\ \mathrm{C} & -0.468 & 2.335 & 0.489 \\ \mathrm{C} & 0.180 & 2.433 & -0.679 \\ \mathrm{C} & -1.612 & 0.319 & -0.242 \\ \mathrm{C} & -0.718 & 0.303 & -1.522 \\ \mathrm{H} & 1.143 & 1.259 & -2.240 \\ \mathrm{H} & 2.238 & 1.190 & 0.074 \\ \mathrm{H} & 0.432 & -0.899 & 1.236 \\ \mathrm{H} & 1.130 & 0.564 & 1.960 \\ \mathrm{H} & -1.328 & 0.903 & 1.883 \\ \mathrm{H} & -0.807 & 3.201 & 1.081 \\ \mathrm{H} & 0.456 & 3.389 & -1.154 \\ \mathrm{H} & -1.282 & 0.751 & -2.373 \\ \mathrm{H} & -0.471 & -0.735 & -1.831 \\ \mathrm{H} & -2.448 & 1.029 & -0.427 \\ & & & \end{array}$

41

$\begin{array}{lrrr}\text { Structure 4, Gd } & \text { Complex } & \\ \mathrm{P} & -2.476 & -0.926 & 0.211 \\ \mathrm{O} & -1.541 & -2.045 & 0.517 \\ \mathrm{C} & -3.545 & -1.269 & -1.201 \\ \mathrm{C} & -3.637 & -0.552 & 1.536 \\ \mathrm{P} & 2.443 & -0.631 & -0.124 \\ \mathrm{O} & 1.663 & -1.892 & -0.266 \\ \mathrm{C} & 3.534 & -0.636 & 1.312 \\ \mathrm{C} & 3.558 & -0.297 & -1.499 \\ \mathrm{H} & -4.161 & -2.170 & -1.001 \\ \mathrm{H} & -2.932 & -1.455 & -2.107 \\ \mathrm{H} & -4.220 & -0.411 & -1.395 \\ \mathrm{H} & -4.297 & -1.425 & 1.718 \\ \mathrm{H} & -4.264 & 0.321 & 1.266 \\ \mathrm{H} & -3.090 & -0.326 & 2.474 \\ \mathrm{H} & 4.262 & -1.469 & 1.232 \\ \mathrm{H} & 2.942 & -0.772 & 2.240 \\ \mathrm{H} & 4.092 & 0.321 & 1.380 \\ \mathrm{H} & 4.327 & -1.093 & -1.565 \\ \mathrm{H} & 4.066 & 0.679 & -1.357 \\ \mathrm{H} & 2.995 & -0.274 & -2.454 \\ \mathrm{Gd} & 0.161 & -3.521 & 0.234 \\ \mathrm{C} & -0.940 & 1.362 & 0.926 \\ \mathrm{C} & -1.650 & 0.637 & -0.245 \\ \mathrm{C} & -0.604 & 0.429 & -1.377 \\ \mathrm{C} & 0.632 & 1.321 & -1.134 \\ \mathrm{C} & 1.419 & 0.861 & 0.119 \\ \mathrm{C} & 0.398 & 0.678 & 1.279 \\ \mathrm{C} & 0.139 & 2.761 & -0.875 \\ \mathrm{C} & -0.634 & 2.804 & 0.467 \\ \mathrm{H} & -1.599 & 1.396 & 1.825 \\ \mathrm{H} & -2.437 & 1.324 & -0.624 \\ \mathrm{H} & -1.064 & 0.678 & -2.361 \\ \mathrm{H} & -0.285 & -0.634 & -1.448 \\ \mathrm{H} & 1.289 & 1.316 & -2.034 \\ \mathrm{H} & 2.108 & 1.689 & 0.393 \\ \mathrm{H} & 0.814 & 1.117 & 2.215 \\ \mathrm{H} & 0.218 & -0.397 & 1.498 \\ \mathrm{H} & 0.998 & 3.471 & -0.847 \\ \mathrm{H} & -0.520 & 3.090 & -1.711 \\ \mathrm{H} & -0.030 & 3.327 & 1.244 \\ \mathrm{H} & -1.576 & 3.387 & 0.349 \\ & & & \end{array}$




$\begin{array}{lrrr}\text { Structure } 4, & \text { free ligand } & \text { global minimum } \\ \mathrm{P} & 2.548 & -0.389 & -1.294 \\ \mathrm{O} & 3.132 & 0.130 & -2.565 \\ \mathrm{C} & 1.777 & -2.005 & -1.496 \\ \mathrm{C} & 3.801 & -0.726 & -0.036 \\ \mathrm{P} & -2.195 & -1.274 & 0.313 \\ \mathrm{O} & -1.143 & -2.326 & 0.400 \\ \mathrm{C} & -3.434 & -1.592 & -0.959 \\ \mathrm{C} & -3.181 & -1.104 & 1.813 \\ \mathrm{H} & 2.532 & -2.736 & -1.849 \\ \mathrm{H} & 0.967 & -1.961 & -2.250 \\ \mathrm{H} & 1.364 & -2.372 & -0.536 \\ \mathrm{H} & 4.538 & -1.460 & -0.421 \\ \mathrm{H} & 3.333 & -1.142 & 0.880 \\ \mathrm{H} & 4.336 & 0.208 & 0.229 \\ \mathrm{H} & -3.957 & -2.547 & -0.749 \\ \mathrm{H} & -2.951 & -1.665 & -1.955 \\ \mathrm{H} & -4.182 & -0.773 & -0.984 \\ \mathrm{H} & -3.730 & -2.047 & 2.015 \\ \mathrm{H} & -3.916 & -0.281 & 1.703 \\ \mathrm{H} & -2.527 & -0.887 & 2.682 \\ \mathrm{C} & 0.352 & 1.415 & -1.381 \\ \mathrm{C} & 1.468 & 0.835 & -0.474 \\ \mathrm{C} & 0.806 & 0.350 & 0.842 \\ \mathrm{C} & -0.600 & 0.974 & 0.990 \\ \mathrm{C} & -1.575 & 0.402 & -0.068 \\ \mathrm{C} & -0.865 & 0.468 & -1.448 \\ \mathrm{C} & -0.482 & 2.493 & 0.740 \\ \mathrm{C} & -0.116 & 2.742 & -0.745 \\ \mathrm{H} & 0.742 & 1.612 & -2.407 \\ \mathrm{H} & 2.129 & 1.689 & -0.211 \\ \mathrm{H} & 1.442 & 0.639 & 1.711 \\ \mathrm{H} & 0.729 & -0.757 & 0.875 \\ \mathrm{H} & -0.988 & 0.799 & 2.021 \\ \mathrm{H} & -2.458 & 1.076 & -0.104 \\ \mathrm{H} & -1.582 & 0.825 & -2.222 \\ \mathrm{H} & -0.533 & -0.539 & -1.777 \\ \mathrm{H} & -1.438 & 3.008 & 0.992 \\ \mathrm{H} & 0.298 & 2.926 & 1.409 \\ \mathrm{H} & -0.999 & 3.135 & -1.300 \\ \mathrm{H} & 0.680 & 3.518 & -0.821 \\ & & & \end{array}$




\begin{tabular}{lrrr} 
41 & \multicolumn{3}{c}{} \\
$\mathrm{Structure} 5$, & $\mathrm{Gd}$ & Complex & \\
$\mathrm{P}$ & -1.673 & 0.170 & 0.457 \\
$\mathrm{O}$ & -1.481 & -1.305 & 0.376 \\
$\mathrm{C}$ & -3.189 & 0.674 & -0.386 \\
$\mathrm{C}$ & -1.979 & 0.709 & 2.152 \\
$\mathrm{P}$ & 1.951 & -0.551 & 0.864 \\
$\mathrm{O}$ & 1.078 & -1.754 & 0.957 \\
$\mathrm{C}$ & 2.889 & -0.301 & 2.393 \\
$\mathrm{C}$ & 3.287 & -0.759 & -0.334 \\
$\mathrm{H}$ & -4.024 & 0.012 & -0.076 \\
$\mathrm{H}$ & -3.067 & 0.589 & -1.485 \\
$\mathrm{H}$ & -3.468 & 1.716 & -0.130 \\
$\mathrm{H}$ & -2.860 & 0.173 & 2.561 \\
$\mathrm{H}$ & -2.183 & 1.798 & 2.197 \\
$\mathrm{H}$ & -1.105 & 0.478 & 2.794 \\
$\mathrm{H}$ & 3.489 & -1.206 & 2.618 \\
$\mathrm{H}$ & 2.194 & -0.122 & 3.239 \\
$\mathrm{H}$ & 3.576 & 0.564 & 2.306 \\
$\mathrm{H}$ & 3.818 & -1.712 & -0.135 \\
$\mathrm{H}$ & 4.024 & 0.066 & -0.259 \\
$\mathrm{H}$ & 2.896 & -0.793 & -1.369 \\
$\mathrm{Gd}$ & -0.509 & -3.343 & 0.589 \\
$\mathrm{C}$ & 2.236 & 2.055 & -1.330 \\
$\mathrm{C}$ & 1.339 & 1.494 & -2.154 \\
$\mathrm{C}$ & 0.043 & 0.842 & -1.724 \\
$\mathrm{C}$ & -0.244 & 1.159 & -0.220 \\
$\mathrm{C}$ & 1.053 & 1.056 & 0.668 \\
$\mathrm{C}$ & 2.066 & 2.120 & 0.166 \\
$\mathrm{C}$ & 0.050 & -0.634 & -2.163 \\
$\mathrm{C}$ & -0.730 & 2.644 & -0.170 \\
$\mathrm{H}$ & 3.137 & 2.533 & -1.753 \\
$\mathrm{H}$ & 1.540 & 1.508 & -3.239 \\
$\mathrm{H}$ & -0.760 & 1.334 & -2.322 \\
$\mathrm{H}$ & 0.759 & 1.362 & 1.694 \\
$\mathrm{H}$ & 3.056 & 1.989 & 0.656 \\
$\mathrm{H}$ & 1.745 & 3.145 & 0.457 \\
$\mathrm{H}$ & -0.951 & -1.107 & -2.061 \\
$\mathrm{H}$ & 0.322 & -0.734 & -3.238 \\
$\mathrm{H}$ & 0.769 & -1.257 & -1.596 \\
$\mathrm{H}$ & -0.845 & 3.023 & 0.869 \\
$\mathrm{H}$ & -0.034 & 3.343 & -0.682 \\
$\mathrm{H}$ & -1.703 & 2.798 & -0.684
\end{tabular}

\begin{tabular}{|c|c|c|c|}
\hline \multicolumn{4}{|c|}{40} \\
\hline St & ture 5, & free ligand & global minimum \\
\hline $\mathrm{P}$ & -1.372 & 0.042 & -0.081 \\
\hline O & -0.988 & -1.159 & -0.872 \\
\hline $\mathrm{C}$ & -2.664 & 0.989 & -0.921 \\
\hline C & -2.189 & -0.405 & 1.468 \\
\hline $\mathrm{P}$ & 2.193 & -0.877 & 0.760 \\
\hline O & 3.347 & -0.470 & -0.094 \\
\hline $\mathrm{C}$ & 1.360 & -2.355 & 0.157 \\
\hline $\mathrm{C}$ & 2.726 & -1.541 & 2.365 \\
\hline $\mathrm{H}$ & -3.468 & 0.303 & -1.257 \\
\hline $\mathrm{H}$ & -2.250 & 1.502 & -1.812 \\
\hline $\mathrm{H}$ & -3.119 & 1.742 & -0.247 \\
\hline $\mathrm{H}$ & -3.057 & -1.063 & 1.256 \\
\hline $\mathrm{H}$ & -2.557 & 0.493 & 2.002 \\
\hline $\mathrm{H}$ & -1.490 & -0.956 & 2.129 \\
\hline $\mathrm{H}$ & 2.043 & -3.226 & 0.230 \\
\hline $\mathrm{H}$ & 1.088 & -2.240 & -0.910 \\
\hline $\mathrm{H}$ & 0.456 & -2.572 & 0.762 \\
\hline $\mathrm{H}$ & 3.360 & -2.438 & 2.214 \\
\hline $\mathrm{H}$ & 1.847 & -1.828 & 2.977 \\
\hline $\mathrm{H}$ & 3.320 & -0.793 & 2.926 \\
\hline C & 2.562 & 2.489 & 0.597 \\
\hline $\mathrm{C}$ & 1.959 & 2.539 & -0.599 \\
\hline $\mathrm{C}$ & 0.706 & 1.780 & -0.972 \\
\hline $\mathrm{C}$ & 0.047 & 1.179 & 0.312 \\
\hline $\mathrm{C}$ & 1.103 & 0.526 & 1.280 \\
\hline C & 2.058 & 1.663 & 1.753 \\
\hline C & 1.024 & 0.821 & -2.135 \\
\hline C & -0.610 & 2.380 & 1.069 \\
\hline $\mathrm{H}$ & 3.468 & 3.094 & 0.773 \\
\hline $\mathrm{H}$ & 2.401 & 3.174 & -1.387 \\
\hline $\mathrm{H}$ & -0.002 & 2.537 & -1.382 \\
\hline $\mathrm{H}$ & 0.551 & 0.175 & 2.177 \\
\hline $\mathrm{H}$ & 2.931 & 1.247 & 2.303 \\
\hline
\end{tabular}




$\begin{array}{rrrr}\mathrm{H} & 1.554 & 2.326 & 2.491 \\ \mathrm{H} & 0.109 & 0.360 & -2.564 \\ \mathrm{H} & 1.513 & 1.361 & -2.977 \\ \mathrm{H} & 1.708 & -0.002 & -1.850 \\ \mathrm{H} & -0.990 & 2.095 & 2.075 \\ \mathrm{H} & 0.091 & 3.226 & 1.228 \\ \mathrm{H} & -1.461 & 2.828 & 0.514\end{array}$

41

$\begin{array}{lrrr}\text { Structure 6, Gd } & \text { complex } & \\ \mathrm{P} & -1.080 & -1.058 & 1.612 \\ \mathrm{O} & -0.895 & -2.449 & 1.115 \\ \mathrm{C} & -2.684 & -0.780 & 2.390 \\ \mathrm{C} & 0.078 & -0.608 & 2.918 \\ \mathrm{P} & 1.180 & -0.830 & -1.426 \\ \mathrm{O} & 0.816 & -2.236 & -1.092 \\ \mathrm{C} & 2.963 & -0.551 & -1.302 \\ \mathrm{C} & 0.886 & -0.425 & -3.160 \\ \mathrm{H} & -2.798 & -1.445 & 3.270 \\ \mathrm{H} & -3.502 & -1.002 & 1.675 \\ \mathrm{H} & -2.776 & 0.273 & 2.724 \\ \mathrm{H} & -0.085 & -1.252 & 3.807 \\ \mathrm{H} & -0.062 & 0.451 & 3.215 \\ \mathrm{H} & 1.122 & -0.750 & 2.572 \\ \mathrm{H} & 3.502 & -1.243 & -1.981 \\ \mathrm{H} & 3.306 & -0.741 & -0.264 \\ \mathrm{H} & 3.223 & 0.490 & -1.580 \\ \mathrm{H} & 1.369 & -1.186 & -3.807 \\ \mathrm{H} & 1.317 & 0.564 & -3.414 \\ \mathrm{H} & -0.196 & -0.413 & -3.392 \\ \mathrm{Gd} & -0.134 & -4.115 & -0.222 \\ \mathrm{C} & -0.654 & 2.020 & -1.873 \\ \mathrm{C} & -1.780 & 1.289 & -1.783 \\ \mathrm{C} & -2.052 & 0.220 & -0.750 \\ \mathrm{C} & -0.933 & 0.240 & 0.326 \\ \mathrm{C} & 0.496 & 0.427 & -0.267 \\ \mathrm{C} & 0.504 & 1.830 & -0.921 \\ \mathrm{C} & -0.505 & 3.139 & -2.873 \\ \mathrm{C} & -2.350 & -1.126 & -1.434 \\ \mathrm{H} & -2.600 & 1.489 & -2.493 \\ \mathrm{H} & -2.996 & 0.527 & -0.240 \\ \mathrm{H} & -1.114 & 1.181 & 0.891 \\ \mathrm{H} & 1.202 & 0.475 & 0.589 \\ \mathrm{H} & 1.467 & 2.010 & -1.450 \\ \mathrm{H} & 0.443 & 2.617 & -0.134 \\ \mathrm{H} & -0.365 & 4.115 & -2.358 \\ \mathrm{H} & 0.378 & 2.974 & -3.530 \\ \mathrm{H} & -1.394 & 3.236 & -3.534 \\ \mathrm{H} & -2.679 & -1.901 & -0.709 \\ \mathrm{H} & -3.177 & -1.027 & -2.173 \\ \mathrm{H} & -1.483 & -1.539 & -1.985 \\ \mathrm{H} & & & \end{array}$

$\begin{array}{lrrr}40 & & \\ \text { Structure 6, } & \text { free } & \text { ligand } & \text { global minimum } \\ \mathrm{P} & -1.660 & 0.111 & 1.253 \\ \mathrm{O} & -1.485 & -1.109 & 2.089 \\ \mathrm{C} & -3.137 & 0.061 & 0.217 \\ \mathrm{C} & -1.923 & 1.611 & 2.222 \\ \mathrm{P} & 2.184 & -0.310 & 1.534 \\ \mathrm{O} & 2.966 & -1.128 & 0.563 \\ \mathrm{C} & 1.379 & -1.289 & 2.813 \\ \mathrm{C} & 3.246 & 0.723 & 2.578 \\ \mathrm{H} & -4.043 & -0.021 & 0.852 \\ \mathrm{H} & -3.103 & -0.816 & -0.460 \\ \mathrm{H} & -3.214 & 0.983 & -0.395 \\ \mathrm{H} & -2.859 & 1.522 & 2.811 \\ \mathrm{H} & -2.005 & 2.494 & 1.557 \\ \mathrm{H} & -1.081 & 1.768 & 2.926 \\ \mathrm{H} & 2.144 & -1.798 & 3.434 \\ \mathrm{H} & 0.739 & -2.070 & 2.358 \\ \mathrm{H} & 0.767 & -0.640 & 3.473 \\ \mathrm{H} & 3.934 & 0.084 & 3.169 \\ \mathrm{H} & 2.633 & 1.327 & 3.279 \\ \mathrm{H} & 3.857 & 1.408 & 1.957 \\ \mathrm{C} & 1.936 & 0.883 & -1.609 \\ \mathrm{C} & 1.069 & -0.100 & -1.913 \\ \mathrm{C} & -0.104 & -0.523 & -1.060 \\ \mathrm{C} & -0.288 & 0.489 & 0.103 \\ \mathrm{C} & 1.048 & 0.924 & 0.774 \\ & & & \end{array}$




\begin{tabular}{|c|c|c|c|}
\hline$C$ & 1827 & & 0230 \\
\hline C & $\begin{array}{l}1.821 \\
3.051\end{array}$ & $\begin{array}{l}1.686 \\
1.284\end{array}$ & $\begin{array}{l}-0.332 \\
-2.540\end{array}$ \\
\hline $\mathrm{C}$ & 0.011 & -2.011 & -0.685 \\
\hline $\mathrm{H}$ & 1.200 & -0.648 & -2.860 \\
\hline $\mathrm{H}$ & -1.002 & -0.433 & -1.716 \\
\hline $\mathrm{H}$ & -0.641 & 1.414 & -0.404 \\
\hline $\mathrm{H}$ & 0.804 & 1.665 & 1.564 \\
\hline $\mathrm{H}$ & 2.843 & 1.966 & 0.025 \\
\hline $\mathrm{H}$ & 1.315 & 2.648 & -0.565 \\
\hline $\mathrm{H}$ & 2.954 & 2.352 & -2.839 \\
\hline $\mathrm{H}$ & 4.043 & 1.158 & -2.052 \\
\hline $\mathrm{H}$ & 3.062 & 0.678 & -3.473 \\
\hline $\mathrm{H}$ & -0.880 & -2.374 & -0.129 \\
\hline $\mathrm{H}$ & 0.088 & -2.648 & -1.595 \\
\hline $\mathrm{H}$ & 0.901 & -2.238 & -0.066 \\
\hline 53 & & & \\
\hline & ture 7, & Gd complex & \\
\hline $\mathrm{P}$ & -2.684 & -1.810 & -0.166 \\
\hline 0 & -1.445 & -2.623 & -0.009 \\
\hline $\mathrm{C}$ & -3.733 & -2.447 & -1.489 \\
\hline $\mathrm{C}$ & -3.741 & -1.925 & 1.292 \\
\hline $\mathrm{P}$ & 2.473 & -1.689 & -0.820 \\
\hline 0 & 1.783 & -2.641 & 0.094 \\
\hline $\mathrm{C}$ & 4.244 & -1.995 & -0.974 \\
\hline $\mathrm{C}$ & 1.898 & -1.811 & -2.525 \\
\hline $\mathrm{H}$ & -3.874 & -3.540 & -1.361 \\
\hline $\mathrm{H}$ & -3.255 & -2.267 & -2.474 \\
\hline $\mathrm{H}$ & -4.731 & -1.966 & -1.477 \\
\hline $\mathrm{H}$ & -3.921 & -2.991 & 1.538 \\
\hline $\mathrm{H}$ & -4.721 & -1.439 & 1.119 \\
\hline $\mathrm{H}$ & -3.248 & -1.445 & 2.162 \\
\hline $\mathrm{H}$ & 4.418 & -3.004 & -1.400 \\
\hline $\mathrm{H}$ & 4.731 & -1.947 & 0.021 \\
\hline $\mathrm{H}$ & 4.711 & -1.241 & -1.640 \\
\hline $\mathrm{H}$ & 2.132 & -2.815 & -2.932 \\
\hline $\mathrm{H}$ & 2.392 & -1.045 & -3.157 \\
\hline $\mathrm{H}$ & 0.800 & -1.664 & -2.572 \\
\hline $\mathrm{Gd}$ & 0.142 & -3.942 & 0.953 \\
\hline $\mathrm{C}$ & 0.867 & 0.614 & -0.466 \\
\hline $\mathrm{C}$ & 2.313 & 0.075 & -0.366 \\
\hline $\mathrm{C}$ & 2.882 & 0.387 & 1.036 \\
\hline $\mathrm{C}$ & 1.958 & -0.047 & 2.183 \\
\hline $\mathrm{C}$ & 0.557 & 0.561 & 2.030 \\
\hline $\mathrm{C}$ & -0.074 & 0.171 & 0.678 \\
\hline $\mathrm{C}$ & -1.539 & 0.708 & 0.552 \\
\hline $\mathrm{C}$ & -2.390 & -0.021 & -0.538 \\
\hline $\mathrm{C}$ & -3.767 & 0.669 & -0.720 \\
\hline $\mathrm{C}$ & -3.675 & 2.178 & -0.959 \\
\hline $\mathrm{C}$ & -2.929 & 2.842 & 0.198 \\
\hline $\mathrm{C}$ & -1.528 & 2.238 & 0.311 \\
\hline $\mathrm{H}$ & 0.938 & 1.724 & -0.462 \\
\hline $\mathrm{H}$ & 0.426 & 0.341 & -1.450 \\
\hline $\mathrm{H}$ & 2.924 & 0.644 & -1.101 \\
\hline $\mathrm{H}$ & 3.887 & -0.075 & 1.164 \\
\hline $\mathrm{H}$ & 3.044 & 1.487 & 1.115 \\
\hline $\mathrm{H}$ & 1.886 & -1.157 & 2.220 \\
\hline $\mathrm{H}$ & 2.398 & 0.270 & 3.157 \\
\hline $\mathrm{H}$ & -0.090 & 0.209 & 2.866 \\
\hline $\mathrm{H}$ & 0.628 & 1.668 & 2.121 \\
\hline $\mathrm{H}$ & -0.115 & -0.934 & 0.667 \\
\hline $\mathrm{H}$ & -2.046 & 0.537 & 1.530 \\
\hline $\mathrm{H}$ & -1.846 & 0.049 & -1.504 \\
\hline $\mathrm{H}$ & -4.311 & 0.217 & -1.580 \\
\hline $\mathrm{H}$ & -4.398 & 0.505 & 0.181 \\
\hline $\mathrm{H}$ & -3.149 & 2.380 & -1.920 \\
\hline $\mathrm{H}$ & -4.698 & 2.614 & -1.052 \\
\hline $\mathrm{H}$ & -2.860 & 3.942 & 0.025 \\
\hline $\mathrm{H}$ & -3.487 & 2.697 & 1.151 \\
\hline $\mathrm{H}$ & -0.982 & 2.754 & 1.133 \\
\hline $\mathrm{H}$ & -0.985 & 2.471 & -0.632 \\
\hline 52 & & & \\
\hline St & ure 7 & free ligand & global minimum \\
\hline $\mathrm{P}$ & 2.257 & -1.828 & 0.992 \\
\hline 0 & 1.026 & -2.521 & 0.517 \\
\hline $\mathrm{C}$ & 3.043 & -2.732 & 2.344 \\
\hline $\mathrm{C}$ & 3.567 & -1.767 & -0.247 \\
\hline $\mathrm{P}$ & -2.055 & -1.629 & -1.439 \\
\hline 0 & -0.894 & -2.116 & -2.237 \\
\hline $\mathrm{C}$ & -3.633 & -1.825 & -2.296 \\
\hline
\end{tabular}




\begin{tabular}{|c|c|c|c|}
\hline $\mathrm{C}$ & -2.355 & -2.550 & 0.083 \\
\hline $\mathrm{H}$ & 3.179 & -3.794 & 2.054 \\
\hline $\mathrm{H}$ & 2.404 & -2.696 & 3.250 \\
\hline $\mathrm{H}$ & 4.040 & -2.309 & 2.581 \\
\hline $\mathrm{H}$ & 3.860 & -2.796 & -0.537 \\
\hline $\mathrm{H}$ & 4.460 & -1.244 & 0.150 \\
\hline $\mathrm{H}$ & 3.216 & -1.236 & -1.155 \\
\hline $\mathrm{H}$ & -3.837 & -2.901 & -2.472 \\
\hline $\mathrm{H}$ & -3.607 & -1.311 & -3.278 \\
\hline $\mathrm{H}$ & -4.460 & -1.401 & -1.691 \\
\hline $\mathrm{H}$ & -2.518 & -3.622 & -0.148 \\
\hline $\mathrm{H}$ & -3.254 & -2.157 & 0.600 \\
\hline $\mathrm{H}$ & -1.490 & -2.467 & 0.770 \\
\hline $\mathrm{C}$ & -0.848 & 0.488 & 0.023 \\
\hline $\mathrm{C}$ & -1.991 & 0.138 & -0.960 \\
\hline $\mathrm{C}$ & -1.914 & 1.070 & -2.189 \\
\hline C & -0.528 & 1.084 & -2.848 \\
\hline $\mathrm{C}$ & 0.556 & 1.463 & -1.832 \\
\hline C & 0.562 & 0.520 & -0.607 \\
\hline $\mathrm{C}$ & 1.700 & 0.908 & 0.393 \\
\hline $\mathrm{C}$ & 1.940 & -0.107 & 1.556 \\
\hline $\mathrm{C}$ & 3.112 & 0.378 & 2.444 \\
\hline $\mathrm{C}$ & 2.887 & 1.788 & 3.001 \\
\hline $\mathrm{C}$ & 2.647 & 2.782 & 1.864 \\
\hline $\mathrm{C}$ & 1.474 & 2.320 & 0.992 \\
\hline $\mathrm{H}$ & -1.070 & 1.491 & 0.450 \\
\hline $\mathrm{H}$ & -0.865 & -0.215 & 0.885 \\
\hline $\mathrm{H}$ & -2.948 & 0.360 & -0.438 \\
\hline $\mathrm{H}$ & -2.689 & 0.797 & -2.941 \\
\hline $\mathrm{H}$ & -2.162 & 2.108 & -1.865 \\
\hline $\mathrm{H}$ & -0.298 & 0.093 & -3.297 \\
\hline $\mathrm{H}$ & -0.523 & 1.815 & -3.690 \\
\hline $\mathrm{H}$ & 1.553 & 1.444 & -2.329 \\
\hline $\mathrm{H}$ & 0.377 & 2.513 & -1.513 \\
\hline $\mathrm{H}$ & 0.785 & -0.496 & -0.998 \\
\hline $\mathrm{H}$ & 2.645 & 0.957 & -0.197 \\
\hline $\mathrm{H}$ & 1.030 & -0.124 & 2.192 \\
\hline $\mathrm{H}$ & 3.252 & -0.308 & 3.309 \\
\hline $\mathrm{H}$ & 4.063 & 0.366 & 1.863 \\
\hline $\mathrm{H}$ & 2.012 & 1.785 & 3.690 \\
\hline $\mathrm{H}$ & 3.770 & 2.106 & 3.603 \\
\hline $\mathrm{H}$ & 2.436 & 3.794 & 2.282 \\
\hline $\mathrm{H}$ & 3.566 & 2.875 & 1.241 \\
\hline $\mathrm{H}$ & 1.329 & 3.071 & 0.186 \\
\hline $\mathrm{H}$ & 0.547 & 2.328 & 1.608 \\
\hline \multicolumn{4}{|l|}{41} \\
\hline St & ture 8, & Gd complex & \\
\hline $\mathrm{P}$ & -1.722 & -0.146 & 0.791 \\
\hline 0 & -1.801 & -1.468 & 0.111 \\
\hline $\mathrm{C}$ & -2.989 & 1.019 & 0.251 \\
\hline $\mathrm{C}$ & -2.008 & -0.243 & 2.569 \\
\hline $\mathrm{P}$ & 1.838 & -1.462 & 0.491 \\
\hline 0 & 0.846 & -2.397 & -0.112 \\
\hline $\mathrm{C}$ & 2.711 & -2.218 & 1.884 \\
\hline $\mathrm{C}$ & 3.212 & -1.085 & -0.617 \\
\hline $\mathrm{H}$ & -3.997 & 0.613 & 0.475 \\
\hline $\mathrm{H}$ & -2.915 & 1.190 & -0.842 \\
\hline $\mathrm{H}$ & -2.872 & 1.990 & 0.775 \\
\hline $\mathrm{H}$ & -3.038 & -0.604 & 2.767 \\
\hline $\mathrm{H}$ & -1.884 & 0.754 & 3.038 \\
\hline $\mathrm{H}$ & -1.295 & -0.952 & 3.036 \\
\hline $\mathrm{H}$ & 3.230 & -3.139 & 1.549 \\
\hline $\mathrm{H}$ & 1.990 & -2.491 & 2.681 \\
\hline $\mathrm{H}$ & 3.463 & -1.521 & 2.306 \\
\hline $\mathrm{H}$ & 3.666 & -2.030 & -0.980 \\
\hline $\mathrm{H}$ & 3.997 & -0.505 & -0.092 \\
\hline $\mathrm{H}$ & 2.870 & -0.508 & -1.498 \\
\hline $\mathrm{Gd}$ & -1.067 & -3.387 & -0.850 \\
\hline $\mathrm{C}$ & 1.524 & 1.909 & -0.911 \\
\hline $\mathrm{C}$ & 2.404 & 1.864 & 0.107 \\
\hline $\mathrm{C}$ & 2.159 & 1.151 & 1.413 \\
\hline $\mathrm{C}$ & 1.094 & 0.041 & 1.253 \\
\hline $\mathrm{C}$ & -0.127 & 0.732 & 0.577 \\
\hline $\mathrm{C}$ & 0.161 & 1.242 & -0.864 \\
\hline $\mathrm{C}$ & 1.855 & 2.667 & -2.175 \\
\hline $\mathrm{C}$ & -0.014 & 0.214 & -1.999 \\
\hline $\mathrm{H}$ & 3.361 & 2.405 & 0.019 \\
\hline $\mathrm{H}$ & 1.827 & 1.899 & 2.168 \\
\hline $\mathrm{H}$ & 3.114 & 0.731 & 1.803 \\
\hline $\mathrm{H}$ & 0.784 & -0.250 & 2.279 \\
\hline
\end{tabular}




$\begin{array}{rrrr}\mathrm{H} & -0.271 & 1.662 & 1.169 \\ \mathrm{H} & -0.577 & 2.054 & -1.066 \\ \mathrm{H} & 1.048 & 3.388 & -2.433 \\ \mathrm{H} & 2.796 & 3.252 & -2.080 \\ \mathrm{H} & 1.987 & 1.979 & -3.038 \\ \mathrm{H} & 0.108 & 0.684 & -2.999 \\ \mathrm{H} & 0.714 & -0.619 & -1.951 \\ \mathrm{H} & -1.030 & -0.236 & -2.005\end{array}$

\begin{tabular}{|c|c|c|c|}
\hline \multicolumn{4}{|l|}{40} \\
\hline $\begin{array}{l}\text { Str } \\
\mathrm{P}\end{array}$ & $\begin{array}{l}\text { ture } 8, \\
-1.716\end{array}$ & $\begin{array}{c}\text { Iree ligand } \\
-2.129\end{array}$ & $\begin{array}{c}g \perp 0.0 \mathrm{~d} \perp \mathrm{min} \\
0.033\end{array}$ \\
\hline 0 & -1.025 & -3.055 & -0.906 \\
\hline $\mathrm{C}$ & -3.312 & -1.548 & -0.578 \\
\hline $\mathrm{C}$ & -2.166 & -2.904 & 1.599 \\
\hline $\mathrm{P}$ & 2.071 & -1.381 & 0.508 \\
\hline O & 2.784 & -0.317 & -0.257 \\
\hline $\mathrm{C}$ & 1.883 & -2.921 & -0.406 \\
\hline $\mathrm{C}$ & 3.040 & -1.986 & 1.914 \\
\hline $\mathrm{H}$ & -3.995 & -2.409 & -0.730 \\
\hline $\mathrm{H}$ & -3.187 & -1.025 & -1.547 \\
\hline $\mathrm{H}$ & -3.776 & -0.851 & 0.149 \\
\hline $\mathrm{H}$ & -2.883 & -3.731 & 1.420 \\
\hline $\mathrm{H}$ & -2.639 & -2.165 & 2.278 \\
\hline $\mathrm{H}$ & -1.267 & -3.324 & 2.094 \\
\hline $\mathrm{H}$ & 2.882 & -3.346 & -0.635 \\
\hline $\mathrm{H}$ & 1.364 & -2.741 & -1.367 \\
\hline $\mathrm{H}$ & 1.316 & -3.662 & 0.193 \\
\hline $\mathrm{H}$ & 3.995 & -2.423 & 1.557 \\
\hline $\mathrm{H}$ & 2.479 & -2.767 & 2.466 \\
\hline $\mathrm{H}$ & 3.278 & -1.158 & 2.611 \\
\hline $\mathrm{C}$ & 0.130 & 1.639 & -0.151 \\
\hline $\mathrm{C}$ & 0.744 & 1.662 & 1.047 \\
\hline $\mathrm{C}$ & 0.757 & 0.510 & 2.022 \\
\hline $\mathrm{C}$ & 0.503 & -0.842 & 1.307 \\
\hline $\mathrm{C}$ & -0.792 & -0.610 & 0.474 \\
\hline $\mathrm{C}$ & -0.633 & 0.434 & -0.667 \\
\hline $\mathrm{C}$ & 0.175 & 2.844 & -1.059 \\
\hline $\mathrm{C}$ & -0.046 & -0.090 & -1.991 \\
\hline $\mathrm{H}$ & 1.265 & 2.579 & 1.370 \\
\hline $\mathrm{H}$ & -0.024 & 0.691 & 2.797 \\
\hline $\mathrm{H}$ & 1.726 & 0.495 & 2.569 \\
\hline $\mathrm{H}$ & 0.271 & -1.587 & 2.097 \\
\hline $\mathrm{H}$ & -1.481 & -0.111 & 1.191 \\
\hline $\mathrm{H}$ & -1.657 & 0.805 & -0.905 \\
\hline $\mathrm{H}$ & -0.847 & 3.139 & -1.386 \\
\hline $\mathrm{H}$ & 0.625 & 3.731 & -0.561 \\
\hline $\mathrm{H}$ & 0.780 & 2.644 & -1.970 \\
\hline $\mathrm{H}$ & -0.113 & 0.674 & -2.797 \\
\hline $\mathrm{H}$ & 1.023 & -0.372 & -1.914 \\
\hline $\mathrm{H}$ & -0.595 & -0.978 & -2.374 \\
\hline 39 & & & \\
\hline Str & ture 9, & Gd complex & \\
\hline P & -1.966 & -0.658 & -0.086 \\
\hline O & -1.343 & -1.962 & 0.270 \\
\hline $\mathrm{C}$ & -2.239 & -0.427 & -1.849 \\
\hline $\mathrm{C}$ & -3.625 & -0.473 & 0.603 \\
\hline $\mathrm{P}$ & 1.974 & -0.779 & -1.012 \\
\hline 0 & 1.218 & -2.019 & -0.691 \\
\hline $\mathrm{C}$ & 3.751 & -0.960 & -0.750 \\
\hline $\mathrm{C}$ & 1.881 & -0.274 & -2.740 \\
\hline $\mathrm{H}$ & -2.940 & -1.199 & -2.229 \\
\hline $\mathrm{H}$ & -1.283 & -0.522 & -2.400 \\
\hline $\mathrm{H}$ & -2.670 & 0.576 & -2.046 \\
\hline $\mathrm{H}$ & -4.280 & -1.290 & 0.239 \\
\hline $\mathrm{H}$ & -4.067 & 0.499 & 0.300 \\
\hline $\mathrm{H}$ & -3.585 & -0.518 & 1.711 \\
\hline $\mathrm{H}$ & 4.148 & -1.769 & -1.396 \\
\hline $\mathrm{H}$ & 3.956 & -1.223 & 0.308 \\
\hline $\mathrm{H}$ & 4.280 & -0.016 & -0.994 \\
\hline $\mathrm{H}$ & 2.275 & -1.081 & -3.392 \\
\hline $\mathrm{H}$ & 2.481 & 0.644 & -2.906 \\
\hline $\mathrm{H}$ & 0.832 & -0.070 & -3.028 \\
\hline $\mathrm{Gd}$ & 0.148 & -3.657 & 0.461 \\
\hline C & 0.149 & 1.353 & -0.239 \\
\hline $\mathrm{C}$ & 1.536 & 0.679 & -0.014 \\
\hline $\mathrm{C}$ & 1.731 & 0.450 & 1.512 \\
\hline $\mathrm{C}$ & 0.685 & 1.264 & 2.302 \\
\hline
\end{tabular}




\begin{tabular}{|c|c|c|c|}
\hline C & 0.654 & 2.670 & 1.722 \\
\hline C & 0.363 & 2.721 & 0.414 \\
\hline $\mathrm{C}$ & -0.703 & 0.646 & 2.044 \\
\hline C & -1.087 & 0.809 & 0.541 \\
\hline $\mathrm{H}$ & -0.074 & 1.492 & -1.322 \\
\hline $\mathrm{H}$ & 2.285 & 1.445 & -0.311 \\
\hline $\mathrm{H}$ & 1.629 & -0.628 & 1.774 \\
\hline $\mathrm{H}$ & 2.759 & 0.756 & 1.814 \\
\hline $\mathrm{H}$ & 0.923 & 1.280 & 3.392 \\
\hline $\mathrm{H}$ & 0.845 & 3.557 & 2.348 \\
\hline $\mathrm{H}$ & 0.291 & 3.657 & -0.165 \\
\hline $\mathrm{H}$ & -1.839 & 1.627 & 0.494 \\
\hline $\mathrm{H}$ & -1.467 & 1.142 & 2.687 \\
\hline $\mathrm{H}$ & -0.685 & -0.426 & 2.348 \\
\hline \multicolumn{4}{|l|}{38} \\
\hline \multicolumn{4}{|c|}{ Structure 9, } \\
\hline $\mathrm{P}$ & 1.874 & -1.274 & 1.171 \\
\hline 0 & 1.088 & -1.577 & 2.400 \\
\hline $\mathrm{C}$ & 3.639 & -1.068 & 1.519 \\
\hline C & 1.959 & -2.628 & -0.019 \\
\hline $\mathrm{P}$ & -1.939 & -1.086 & 0.455 \\
\hline 0 & -1.197 & -2.289 & -0.011 \\
\hline C & -3.592 & -0.965 & -0.265 \\
\hline $\mathrm{C}$ & -2.290 & -1.048 & 2.223 \\
\hline $\mathrm{H}$ & 4.059 & -2.009 & 1.929 \\
\hline $\mathrm{H}$ & 3.790 & -0.261 & 2.265 \\
\hline $\mathrm{H}$ & 4.191 & -0.805 & 0.593 \\
\hline $\mathrm{H}$ & 2.205 & -3.574 & 0.505 \\
\hline $\mathrm{H}$ & 2.748 & -2.435 & -0.774 \\
\hline $\mathrm{H}$ & 0.998 & -2.763 & -0.549 \\
\hline $\mathrm{H}$ & -4.191 & -1.858 & 0.007 \\
\hline $\mathrm{H}$ & -3.525 & -0.908 & -1.370 \\
\hline $\mathrm{H}$ & -4.115 & -0.060 & 0.108 \\
\hline $\mathrm{H}$ & -2.888 & -1.936 & 2.510 \\
\hline $\mathrm{H}$ & -2.862 & -0.134 & 2.482 \\
\hline $\mathrm{H}$ & -1.350 & -1.053 & 2.809 \\
\hline $\mathrm{C}$ & 0.082 & 0.958 & 0.815 \\
\hline $\mathrm{C}$ & -1.178 & 0.510 & 0.019 \\
\hline C & -0.866 & 0.612 & -1.507 \\
\hline C & 0.496 & 1.301 & -1.727 \\
\hline $\mathrm{C}$ & 0.474 & 2.591 & -0.922 \\
\hline $\mathrm{C}$ & 0.249 & 2.418 & 0.388 \\
\hline $\mathrm{C}$ & 1.597 & 0.396 & -1.135 \\
\hline $\mathrm{C}$ & 1.450 & 0.330 & 0.413 \\
\hline $\mathrm{H}$ & -0.086 & 0.915 & 1.917 \\
\hline $\mathrm{H}$ & -1.956 & 1.273 & 0.239 \\
\hline $\mathrm{H}$ & -0.847 & -0.393 & -1.987 \\
\hline $\mathrm{H}$ & -1.671 & 1.189 & -2.020 \\
\hline $\mathrm{H}$ & 0.676 & 1.503 & -2.809 \\
\hline $\mathrm{H}$ & 0.623 & 3.574 & -1.399 \\
\hline $\mathrm{H}$ & 0.196 & 3.242 & 1.119 \\
\hline $\mathrm{H}$ & 2.211 & 1.032 & 0.819 \\
\hline $\mathrm{H}$ & 2.604 & 0.790 & -1.405 \\
\hline $\mathrm{H}$ & 1.516 & -0.611 & -1.598 \\
\hline \multicolumn{4}{|l|}{41} \\
\hline Str & ture 10, & Gd complex & \\
\hline $\mathrm{P}$ & -1.442 & -0.475 & 1.303 \\
\hline 0 & -1.685 & -1.821 & 0.716 \\
\hline C & -2.950 & 0.356 & 1.844 \\
\hline $\mathrm{C}$ & -0.452 & -0.523 & 2.810 \\
\hline $\mathrm{P}$ & 1.277 & -1.017 & -1.264 \\
\hline 0 & 0.385 & -2.200 & -1.101 \\
\hline C & 2.988 & -1.407 & -0.824 \\
\hline $\mathrm{C}$ & 1.462 & -0.517 & -2.989 \\
\hline $\mathrm{H}$ & -3.452 & -0.245 & 2.629 \\
\hline $\mathrm{H}$ & -3.649 & 0.475 & 0.992 \\
\hline $\mathrm{H}$ & -2.714 & 1.357 & 2.258 \\
\hline $\mathrm{H}$ & -0.994 & -1.082 & 3.600 \\
\hline $\mathrm{H}$ & -0.250 & 0.505 & 3.175 \\
\hline $\mathrm{H}$ & 0.513 & -1.035 & 2.619 \\
\hline $\mathrm{H}$ & 3.358 & -2.244 & -1.451 \\
\hline $\mathrm{H}$ & 3.046 & -1.714 & 0.240 \\
\hline $\mathrm{H}$ & 3.649 & -0.531 & -0.979 \\
\hline $\mathrm{H}$ & 1.756 & -1.395 & -3.600 \\
\hline $\mathrm{H}$ & 2.248 & 0.256 & -3.098 \\
\hline $\mathrm{H}$ & 0.512 & -0.120 & -3.395 \\
\hline $\mathrm{Gd}$ & -1.331 & -3.620 & -0.617 \\
\hline C & 0.684 & 2.301 & -1.770 \\
\hline C & -0.621 & 2.041 & -1.937 \\
\hline
\end{tabular}




$\begin{array}{lrrr}\mathrm{C} & -1.447 & 1.129 & -1.060 \\ \mathrm{C} & -0.611 & 0.714 & 0.182 \\ \mathrm{C} & 0.882 & 0.396 & -0.143 \\ \mathrm{C} & 1.538 & 1.704 & -0.677 \\ \mathrm{C} & -2.072 & -0.001 & -1.896 \\ \mathrm{C} & 1.794 & 2.750 & 0.425 \\ \mathrm{H} & 1.183 & 3.002 & -2.461 \\ \mathrm{H} & -1.156 & 2.531 & -2.768 \\ \mathrm{H} & -2.296 & 1.751 & -0.690 \\ \mathrm{H} & -0.566 & 1.637 & 0.795 \\ \mathrm{H} & 1.381 & 0.165 & 0.821 \\ \mathrm{H} & 2.531 & 1.457 & -1.117 \\ \mathrm{H} & -1.322 & -0.700 & -2.318 \\ \mathrm{H} & -2.646 & 0.406 & -2.758 \\ \mathrm{H} & -2.793 & -0.609 & -1.307 \\ \mathrm{H} & 2.365 & 3.620 & 0.029 \\ \mathrm{H} & 0.856 & 3.157 & 0.862 \\ \mathrm{H} & 2.394 & 2.325 & 1.261\end{array}$

40

$\begin{array}{lrrr}\text { Structure 10, } & \text { free ligand } & \text { global minimum } \\ \mathrm{P} & 1.645 & -1.412 & -0.797 \\ \mathrm{O} & 1.954 & -2.000 & 0.535 \\ \mathrm{C} & 3.112 & -0.846 & -1.684 \\ \mathrm{C} & 0.931 & -2.596 & -1.957 \\ \mathrm{P} & -1.455 & -0.565 & 1.336 \\ \mathrm{O} & -1.562 & 0.672 & 2.162 \\ \mathrm{C} & -0.513 & -1.877 & 2.131 \\ \mathrm{C} & -3.044 & -1.421 & 1.163 \\ \mathrm{H} & 3.798 & -1.699 & -1.864 \\ \mathrm{H} & 3.651 & -0.082 & -1.088 \\ \mathrm{H} & 2.830 & -0.404 & -2.661 \\ \mathrm{H} & 1.655 & -3.412 & -2.158 \\ \mathrm{H} & 0.679 & -2.098 & -2.915 \\ \mathrm{H} & 0.010 & -3.045 & -1.532 \\ \mathrm{H} & -1.027 & -2.200 & 3.058 \\ \mathrm{H} & 0.494 & -1.514 & 2.413 \\ \mathrm{H} & -0.421 & -2.754 & 1.458 \\ \mathrm{H} & -3.424 & -1.722 & 2.161 \\ \mathrm{H} & -2.930 & -2.331 & 0.539 \\ \mathrm{H} & -3.798 & -0.759 & 0.692 \\ \mathrm{C} & -1.228 & 2.230 & -0.561 \\ \mathrm{C} & 0.047 & 2.403 & -0.182 \\ \mathrm{C} & 1.085 & 1.308 & -0.119 \\ \mathrm{C} & 0.515 & 0.029 & -0.790 \\ \mathrm{C} & -0.969 & -0.277 & -0.420 \\ \mathrm{C} & -1.828 & 0.905 & -0.972 \\ \mathrm{C} & 1.634 & 1.168 & 1.311 \\ \mathrm{C} & -2.014 & 0.857 & -2.501 \\ \mathrm{H} & -1.905 & 3.102 & -0.572 \\ \mathrm{H} & 0.379 & 3.412 & 0.115 \\ \mathrm{H} & 1.935 & 1.659 & -0.751 \\ \mathrm{H} & 0.471 & 0.297 & -1.866 \\ \mathrm{H} & -1.272 & -1.185 & -0.981 \\ \mathrm{H} & -2.848 & 0.852 & -0.527 \\ \mathrm{H} & 0.861 & 0.873 & 2.048 \\ \mathrm{H} & 2.062 & 2.130 & 1.671 \\ \mathrm{H} & 2.454 & 0.421 & 1.374 \\ \mathrm{H} & -2.725 & 1.643 & -2.845 \\ \mathrm{H} & -1.066 & 1.019 & -3.058 \\ \mathrm{H} & -2.431 & -0.121 & -2.831 \\ & & & \end{array}$

41

$\begin{array}{lrrr}\text { Structure 11, Gd complex } & \\ \mathrm{P} & -2.549 & 0.388 & -1.050 \\ \mathrm{O} & -1.759 & -0.871 & -0.940 \\ \mathrm{C} & -2.996 & 0.809 & -2.746 \\ \mathrm{C} & -4.148 & 0.295 & -0.220 \\ \mathrm{P} & 2.346 & -0.764 & -0.435 \\ \mathrm{O} & 1.266 & -1.732 & -0.092 \\ \mathrm{C} & 4.004 & -1.443 & -0.219 \\ \mathrm{C} & 2.331 & -0.281 & -2.170 \\ \mathrm{H} & -3.632 & 0.010 & -3.179 \\ \mathrm{H} & -2.084 & 0.908 & -3.369 \\ \mathrm{H} & -3.556 & 1.766 & -2.773 \\ \mathrm{H} & -4.770 & -0.497 & -0.685 \\ \mathrm{H} & -4.684 & 1.263 & -0.300 \\ \mathrm{H} & -4.011 & 0.048 & 0.852 \\ \mathrm{H} & 4.132 & -2.343 & -0.854 \\ \mathrm{H} & 4.162 & -1.731 & 0.840 \\ \mathrm{H} & 4.770 & -0.693 & -0.504\end{array}$




\begin{tabular}{|c|c|c|c|}
\hline $\mathrm{H}$ & 2.553 & -1.161 & -2.808 \\
\hline $\mathrm{H}$ & 3.095 & 0.500 & -2.364 \\
\hline $\mathrm{H}$ & 1.333 & 0.107 & -2.454 \\
\hline $\mathrm{Gd}$ & -0.747 & -2.778 & -0.208 \\
\hline $\mathrm{C}$ & -0.521 & 2.234 & -1.175 \\
\hline $\mathrm{C}$ & 0.735 & 2.121 & -0.719 \\
\hline $\mathrm{C}$ & 1.109 & 1.610 & 0.652 \\
\hline $\mathrm{C}$ & -0.095 & 0.915 & 1.333 \\
\hline $\mathrm{C}$ & -1.364 & 1.760 & 1.120 \\
\hline $\mathrm{C}$ & -1.747 & 1.884 & -0.370 \\
\hline $\mathrm{C}$ & 2.381 & 0.732 & 0.596 \\
\hline $\mathrm{C}$ & 0.146 & 0.690 & 2.837 \\
\hline $\mathrm{H}$ & -0.683 & 2.644 & -2.187 \\
\hline $\mathrm{H}$ & 1.563 & 2.459 & -1.365 \\
\hline $\mathrm{H}$ & 1.359 & 2.514 & 1.256 \\
\hline $\mathrm{H}$ & -0.259 & -0.076 & 0.866 \\
\hline $\mathrm{H}$ & -1.191 & 2.778 & 1.540 \\
\hline $\mathrm{H}$ & -2.214 & 1.332 & 1.699 \\
\hline $\mathrm{H}$ & -2.459 & 2.732 & -0.465 \\
\hline $\mathrm{H}$ & 3.230 & 1.353 & 0.237 \\
\hline $\mathrm{H}$ & 2.659 & 0.418 & 1.622 \\
\hline $\mathrm{H}$ & 1.003 & 0.006 & 3.025 \\
\hline $\mathrm{H}$ & 0.356 & 1.644 & 3.369 \\
\hline $\mathrm{H}$ & -0.741 & 0.227 & 3.324 \\
\hline \multicolumn{4}{|c|}{40} \\
\hline \multicolumn{4}{|c|}{ Str } \\
\hline $\mathrm{P}$ & -3.113 & -1.394 & 0.333 \\
\hline $\mathrm{O}$ & -4.218 & -1.164 & 1.309 \\
\hline $\mathrm{C}$ & -1.626 & -2.064 & 1.094 \\
\hline $\mathrm{C}$ & -3.519 & -2.664 & -0.886 \\
\hline $\mathrm{P}$ & 2.085 & -0.088 & 0.518 \\
\hline 0 & 1.388 & -0.543 & 1.754 \\
\hline $\mathrm{C}$ & 3.827 & 0.303 & 0.790 \\
\hline $\mathrm{C}$ & 2.167 & -1.338 & -0.777 \\
\hline $\mathrm{H}$ & -1.825 & -3.088 & 1.471 \\
\hline $\mathrm{H}$ & -1.315 & -1.438 & 1.953 \\
\hline $\mathrm{H}$ & -0.800 & -2.115 & 0.357 \\
\hline $\mathrm{H}$ & -3.819 & -3.600 & -0.371 \\
\hline $\mathrm{H}$ & -2.646 & -2.884 & -1.533 \\
\hline $\mathrm{H}$ & -4.363 & -2.327 & -1.523 \\
\hline $\mathrm{H}$ & 4.363 & -0.591 & 1.170 \\
\hline $\mathrm{H}$ & 3.923 & 1.117 & 1.537 \\
\hline $\mathrm{H}$ & 4.304 & 0.629 & -0.157 \\
\hline $\mathrm{H}$ & 2.759 & -2.207 & -0.426 \\
\hline $\mathrm{H}$ & 2.650 & -0.924 & -1.686 \\
\hline $\mathrm{H}$ & 1.151 & -1.694 & -1.040 \\
\hline $\mathrm{C}$ & -1.608 & -0.153 & -1.632 \\
\hline $\mathrm{C}$ & -0.430 & 0.485 & -1.584 \\
\hline $\mathrm{C}$ & -0.061 & 1.523 & -0.550 \\
\hline $\mathrm{C}$ & -1.007 & 1.431 & 0.672 \\
\hline $\mathrm{C}$ & -2.463 & 1.350 & 0.176 \\
\hline $\mathrm{C}$ & -2.743 & 0.088 & -0.670 \\
\hline $\mathrm{C}$ & 1.442 & 1.456 & -0.190 \\
\hline $\mathrm{C}$ & -0.841 & 2.633 & 1.619 \\
\hline $\mathrm{H}$ & -1.792 & -0.859 & -2.460 \\
\hline $\mathrm{H}$ & 0.309 & 0.291 & -2.380 \\
\hline $\mathrm{H}$ & -0.235 & 2.512 & -1.035 \\
\hline $\mathrm{H}$ & -0.782 & 0.509 & 1.247 \\
\hline $\mathrm{H}$ & -2.677 & 2.255 & -0.440 \\
\hline $\mathrm{H}$ & -3.168 & 1.396 & 1.037 \\
\hline $\mathrm{H}$ & -3.645 & 0.304 & -1.283 \\
\hline $\mathrm{H}$ & 2.039 & 1.669 & -1.102 \\
\hline $\mathrm{H}$ & 1.687 & 2.273 & 0.519 \\
\hline $\mathrm{H}$ & 0.171 & 2.669 & 2.077 \\
\hline $\mathrm{H}$ & -1.004 & 3.600 & 1.092 \\
\hline $\mathrm{H}$ & -1.568 & 2.590 & 2.460 \\
\hline \multicolumn{4}{|c|}{38} \\
\hline \multicolumn{4}{|c|}{ St } \\
\hline $\mathrm{P}$ & 1.991 & -0.912 & -0.179 \\
\hline 0 & 1.097 & -1.925 & -0.801 \\
\hline $\mathrm{C}$ & 2.208 & -1.148 & 1.594 \\
\hline $\mathrm{C}$ & 3.671 & -0.963 & -0.834 \\
\hline P & -2.007 & -0.361 & 0.284 \\
\hline 0 & -1.441 & -1.730 & 0.149 \\
\hline $\mathrm{C}$ & -3.576 & -0.171 & -0.587 \\
\hline $\mathrm{C}$ & -2.402 & 0.109 & 1.978 \\
\hline $\mathrm{H}$ & 2.652 & -2.145 & 1.792 \\
\hline $\mathrm{H}$ & 1.226 & -1.091 & 2.107 \\
\hline $\mathrm{H}$ & 2.877 & -0.368 & 2.010 \\
\hline
\end{tabular}




\begin{tabular}{|c|c|c|c|}
\hline $\mathrm{H}$ & 4.104 & -1.974 & -0.689 \\
\hline $\mathrm{H}$ & 4.314 & -0.222 & -0.316 \\
\hline $\mathrm{H}$ & 3.663 & -0.733 & -1.919 \\
\hline $\mathrm{H}$ & -4.314 & -0.903 & -0.202 \\
\hline $\mathrm{H}$ & -3.431 & -0.351 & -1.672 \\
\hline $\mathrm{H}$ & -3.980 & 0.852 & -0.444 \\
\hline $\mathrm{H}$ & -3.175 & -0.571 & 2.389 \\
\hline $\mathrm{H}$ & -2.785 & 1.149 & 2.011 \\
\hline $\mathrm{H}$ & -1.497 & 0.039 & 2.616 \\
\hline $\mathrm{Gd}$ & -0.509 & -3.525 & -0.887 \\
\hline $\mathrm{C}$ & 0.285 & 1.416 & 0.391 \\
\hline $\mathrm{C}$ & 1.507 & 0.826 & -0.354 \\
\hline $\mathrm{C}$ & 1.027 & 1.304 & -1.738 \\
\hline $\mathrm{C}$ & 0.910 & 2.835 & -1.481 \\
\hline $\mathrm{C}$ & 0.486 & 2.914 & 0.022 \\
\hline $\mathrm{C}$ & -0.990 & 0.982 & -0.404 \\
\hline $\mathrm{C}$ & -0.409 & 0.728 & -1.826 \\
\hline $\mathrm{H}$ & 0.254 & 1.239 & 1.490 \\
\hline $\mathrm{H}$ & 2.403 & 1.415 & -0.064 \\
\hline $\mathrm{H}$ & 1.687 & 1.038 & -2.597 \\
\hline $\mathrm{H}$ & 1.297 & 3.367 & 0.639 \\
\hline $\mathrm{H}$ & 1.890 & 3.342 & -1.641 \\
\hline $\mathrm{H}$ & -0.433 & 3.526 & 0.174 \\
\hline $\mathrm{H}$ & 0.160 & 3.309 & -2.153 \\
\hline $\mathrm{H}$ & -1.660 & 1.865 & -0.466 \\
\hline $\mathrm{H}$ & -1.008 & 1.236 & -2.616 \\
\hline $\mathrm{H}$ & -0.381 & -0.355 & -2.078 \\
\hline \multicolumn{4}{|c|}{37} \\
\hline \multicolumn{4}{|c|}{ Structure 12 , } \\
\hline $\mathrm{P}$ & 1.908 & -1.167 & -0.304 \\
\hline O & 1.221 & -1.798 & -1.466 \\
\hline $\mathrm{C}$ & 2.203 & -2.270 & 1.090 \\
\hline $\mathrm{C}$ & 3.568 & -0.585 & -0.711 \\
\hline $\mathrm{P}$ & -1.914 & -1.690 & -0.176 \\
\hline O & -1.089 & -2.709 & 0.534 \\
\hline $\mathrm{C}$ & -2.214 & -2.050 & -1.916 \\
\hline $\mathrm{C}$ & -3.597 & -1.591 & 0.478 \\
\hline $\mathrm{H}$ & 2.793 & -3.150 & 0.761 \\
\hline $\mathrm{H}$ & 1.245 & -2.626 & 1.517 \\
\hline $\mathrm{H}$ & 2.768 & -1.741 & 1.886 \\
\hline $\mathrm{H}$ & 4.193 & -1.433 & -1.059 \\
\hline $\mathrm{H}$ & 4.051 & -0.130 & 0.179 \\
\hline $\mathrm{H}$ & 3.520 & 0.174 & -1.518 \\
\hline $\mathrm{H}$ & -2.777 & -3.001 & -2.013 \\
\hline $\mathrm{H}$ & -1.256 & -2.157 & -2.464 \\
\hline $\mathrm{H}$ & -2.806 & -1.237 & -2.382 \\
\hline $\mathrm{H}$ & -4.093 & -2.579 & 0.399 \\
\hline $\mathrm{H}$ & -4.193 & -0.847 & -0.090 \\
\hline $\mathrm{H}$ & -3.573 & -1.291 & 1.546 \\
\hline $\mathrm{C}$ & -0.259 & 0.229 & 1.115 \\
\hline $\mathrm{C}$ & 1.110 & 0.309 & 0.396 \\
\hline $\mathrm{C}$ & 0.630 & 1.361 & -0.624 \\
\hline $\mathrm{C}$ & 0.150 & 2.492 & 0.327 \\
\hline $\mathrm{C}$ & -0.399 & 1.711 & 1.561 \\
\hline $\mathrm{C}$ & -1.325 & 0.028 & -0.012 \\
\hline $\mathrm{C}$ & -0.625 & 0.690 & -1.237 \\
\hline $\mathrm{H}$ & -0.317 & -0.487 & 1.967 \\
\hline $\mathrm{H}$ & 1.847 & 0.774 & 1.086 \\
\hline $\mathrm{H}$ & 1.388 & 1.693 & -1.372 \\
\hline $\mathrm{H}$ & 0.223 & 1.911 & 2.464 \\
\hline $\mathrm{H}$ & 1.000 & 3.150 & 0.623 \\
\hline $\mathrm{H}$ & -1.450 & 1.988 & 1.806 \\
\hline $\mathrm{H}$ & -0.631 & 3.128 & -0.149 \\
\hline $\mathrm{H}$ & -2.213 & 0.646 & 0.235 \\
\hline $\mathrm{H}$ & -1.285 & 1.437 & -1.733 \\
\hline $\mathrm{H}$ & -0.333 & -0.050 & -2.012 \\
\hline \multicolumn{4}{|c|}{44} \\
\hline \multicolumn{4}{|c|}{ Str } \\
\hline $\mathrm{C}$ & -3.045 & -1.385 & 1.124 \\
\hline $\mathrm{P}$ & 2.692 & -1.845 & -0.432 \\
\hline $\mathrm{P}$ & -2.467 & -0.731 & -0.452 \\
\hline 0 & 1.754 & -2.730 & -1.178 \\
\hline $\mathrm{C}$ & 3.504 & -2.651 & 0.960 \\
\hline $\mathrm{C}$ & 4.068 & -1.235 & -1.422 \\
\hline 0 & -1.518 & -1.628 & -1.171 \\
\hline $\mathrm{C}$ & -4.031 & -0.519 & -1.336 \\
\hline $\mathrm{H}$ & -3.678 & -2.279 & 0.952 \\
\hline $\mathrm{H}$ & -3.644 & -0.623 & 1.664 \\
\hline $\mathrm{H}$ & -2.191 & -1.690 & 1.760 \\
\hline
\end{tabular}




$\begin{array}{lrrr}\mathrm{H} & 4.149 & -3.476 & 0.596 \\ \mathrm{H} & 2.750 & -3.076 & 1.653 \\ \mathrm{H} & 4.132 & -1.925 & 1.514 \\ \mathrm{H} & 4.708 & -2.083 & -1.743 \\ \mathrm{H} & 4.684 & -0.526 & -0.832 \\ \mathrm{H} & 3.692 & -0.718 & -2.328 \\ \mathrm{H} & -4.530 & -1.502 & -1.464 \\ \mathrm{H} & -3.849 & -0.086 & -2.341 \\ \mathrm{H} & -4.708 & 0.154 & -0.772 \\ \mathrm{Gd} & -0.273 & -3.355 & -1.985 \\ \mathrm{C} & 0.495 & 1.707 & -0.357 \\ \mathrm{C} & 1.248 & 0.476 & -0.888 \\ \mathrm{C} & 1.849 & -0.373 & 0.252 \\ \mathrm{C} & 0.722 & -0.809 & 1.213 \\ \mathrm{C} & -0.107 & 0.379 & 1.725 \\ \mathrm{C} & -0.627 & 1.330 & 0.635 \\ \mathrm{C} & -1.889 & 0.988 & -0.231 \\ \mathrm{C} & -1.578 & 1.625 & -1.628 \\ \mathrm{C} & -0.282 & 2.441 & -1.462 \\ \mathrm{H} & 1.225 & 2.398 & 0.128 \\ \mathrm{H} & 2.057 & 0.823 & -1.571 \\ \mathrm{H} & 0.564 & -0.148 & -1.505 \\ \mathrm{H} & 2.583 & 0.245 & 0.812 \\ \mathrm{H} & 1.157 & -1.342 & 2.089 \\ \mathrm{H} & 0.055 & -1.541 & 0.712 \\ \mathrm{H} & -0.953 & 0.024 & 2.355 \\ \mathrm{H} & 0.544 & 0.968 & 2.411 \\ \mathrm{H} & -0.893 & 2.275 & 1.168 \\ \mathrm{H} & -2.737 & 1.552 & 0.214 \\ \mathrm{H} & -1.416 & 0.844 & -2.405 \\ \mathrm{H} & -2.416 & 2.271 & -1.980 \\ \mathrm{H} & 0.296 & 2.514 & -2.411 \\ \mathrm{H} & -0.527 & 3.476 & -1.130\end{array}$

43

$\begin{array}{lrrr}\text { Structure 13, } & \text { free ligand } & \text { global minimum } \\ \mathrm{C} & 2.880 & 1.042 & -2.086 \\ \mathrm{P} & -2.934 & -0.646 & -1.638 \\ \mathrm{P} & 2.437 & -0.439 & -1.157 \\ \mathrm{O} & -3.669 & 0.331 & -2.493 \\ \mathrm{C} & -4.042 & -1.710 & -0.691 \\ \mathrm{C} & -1.946 & -1.814 & -2.591 \\ \mathrm{O} & 1.427 & -1.303 & -1.830 \\ \mathrm{C} & 4.052 & -1.238 & -0.995 \\ \mathrm{H} & 3.430 & 0.760 & -3.007 \\ \mathrm{H} & 3.527 & 1.704 & -1.474 \\ \mathrm{H} & 1.974 & 1.602 & -2.389 \\ \mathrm{H} & -4.770 & -2.196 & -1.372 \\ \mathrm{H} & -4.601 & -1.110 & 0.055 \\ \mathrm{H} & -3.474 & -2.503 & -0.163 \\ \mathrm{H} & -2.598 & -2.373 & -3.293 \\ \mathrm{H} & -1.444 & -2.544 & -1.925 \\ \mathrm{H} & -1.177 & -1.277 & -3.182 \\ \mathrm{H} & 4.452 & -1.493 & -1.998 \\ \mathrm{H} & 3.962 & -2.174 & -0.407 \\ \mathrm{H} & 4.770 & -0.563 & -0.486 \\ \mathrm{C} & -0.249 & -0.088 & 1.549 \\ \mathrm{C} & -1.099 & -0.823 & 0.498 \\ \mathrm{C} & -1.845 & 0.169 & -0.415 \\ \mathrm{C} & -0.822 & 1.078 & -1.127 \\ \mathrm{C} & 0.107 & 1.792 & -0.132 \\ \mathrm{C} & 0.774 & 0.879 & 0.911 \\ \mathrm{C} & 2.038 & 0.019 & 0.565 \\ \mathrm{C} & 1.873 & -1.274 & 1.433 \\ \mathrm{C} & 0.657 & -1.039 & 2.348 \\ \mathrm{H} & -0.927 & 0.468 & 2.241 \\ \mathrm{H} & -1.826 & -1.478 & 1.030 \\ \mathrm{H} & -0.454 & -1.493 & -0.109 \\ \mathrm{H} & -2.483 & 0.812 & 0.230 \\ \mathrm{H} & -1.355 & 1.846 & -1.735 \\ \mathrm{H} & -0.219 & 0.485 & -1.846 \\ \mathrm{H} & 0.880 & 2.383 & -0.670 \\ \mathrm{H} & -0.508 & 2.544 & 0.416 \\ \mathrm{H} & 1.105 & 1.559 & 1.733 \\ \mathrm{H} & 2.916 & 0.578 & 0.957 \\ \mathrm{H} & 1.680 & -2.169 & 0.799 \\ \mathrm{H} & 2.790 & -1.486 & 2.031 \\ \mathrm{H} & 0.141 & -1.989 & 2.619 \\ \mathrm{H} & 0.985 & -0.547 & 3.293 \\ & & & \end{array}$

41 


\begin{tabular}{|c|c|c|c|}
\hline \multicolumn{4}{|c|}{1} \\
\hline $\mathrm{C}$ & 1.803 & -2.263 & -0.467 \\
\hline P & -1.991 & -1.010 & 0.472 \\
\hline $\mathrm{P}$ & 1.614 & -0.578 & 0.152 \\
\hline 0 & -1.128 & -1.292 & 1.653 \\
\hline $\mathrm{C}$ & -3.003 & -2.460 & 0.095 \\
\hline $\mathrm{C}$ & -3.214 & 0.251 & 0.886 \\
\hline 0 & 1.339 & -0.535 & 1.615 \\
\hline $\mathrm{C}$ & 3.230 & 0.139 & -0.217 \\
\hline $\mathrm{H}$ & 2.657 & -2.757 & 0.039 \\
\hline $\mathrm{H}$ & 1.991 & -2.263 & -1.560 \\
\hline $\mathrm{H}$ & 0.888 & -2.854 & -0.259 \\
\hline $\mathrm{H}$ & -3.441 & -2.860 & 1.032 \\
\hline $\mathrm{H}$ & -2.377 & -3.254 & -0.362 \\
\hline $\mathrm{H}$ & -3.837 & -2.220 & -0.591 \\
\hline $\mathrm{H}$ & -3.835 & -0.102 & 1.735 \\
\hline $\mathrm{H}$ & -3.888 & 0.472 & 0.037 \\
\hline $\mathrm{H}$ & -2.714 & 1.188 & 1.201 \\
\hline $\mathrm{H}$ & 3.991 & -0.292 & 0.465 \\
\hline $\mathrm{H}$ & 3.209 & 1.237 & -0.063 \\
\hline $\mathrm{H}$ & 3.541 & -0.082 & -1.258 \\
\hline $\mathrm{Gd}$ & 0.165 & -0.962 & 3.502 \\
\hline $\mathrm{C}$ & -2.087 & 1.537 & -1.795 \\
\hline $\mathrm{C}$ & -1.297 & 2.200 & -0.934 \\
\hline $\mathrm{C}$ & -0.110 & 1.586 & -0.234 \\
\hline $\mathrm{C}$ & 0.288 & 0.235 & -0.875 \\
\hline $\mathrm{C}$ & -1.022 & -0.628 & -1.077 \\
\hline $\mathrm{C}$ & -1.875 & 0.086 & -2.174 \\
\hline $\mathrm{C}$ & 0.963 & 0.534 & -2.247 \\
\hline $\mathrm{C}$ & -3.196 & -0.596 & -2.570 \\
\hline $\mathrm{H}$ & -2.930 & 2.067 & -2.272 \\
\hline $\mathrm{H}$ & -1.522 & 3.254 & -0.696 \\
\hline $\mathrm{H}$ & -0.353 & 1. 464 & 0.845 \\
\hline $\mathrm{H}$ & 0.747 & 2.295 & -0.283 \\
\hline $\mathrm{H}$ & -0.709 & -1.603 & -1.505 \\
\hline $\mathrm{H}$ & -1.285 & 0.087 & -3.117 \\
\hline $\mathrm{H}$ & 1.184 & -0.394 & -2.821 \\
\hline $\mathrm{H}$ & 1.918 & 1.091 & -2.141 \\
\hline $\mathrm{H}$ & 0.350 & 1.185 & -2.905 \\
\hline $\mathrm{H}$ & -3.050 & -1.679 & -2.777 \\
\hline $\mathrm{H}$ & -3.610 & -0.150 & -3.502 \\
\hline $\mathrm{H}$ & -3.991 & -0.493 & -1.803 \\
\hline 40 & & & \\
\hline St & ure 14, & free ligand & lobal minimu \\
\hline $\mathrm{C}$ & 3.267 & 0.486 & 0.307 \\
\hline $\mathrm{P}$ & -1.735 & -1.018 & 0.759 \\
\hline $\mathrm{P}$ & 1.735 & -0.480 & 0.224 \\
\hline O & $-1 \cdot 328$ & -0.506 & 2.098 \\
\hline $\mathrm{C}$ & -1.222 & -2.731 & 0.447 \\
\hline $\mathrm{C}$ & -3.512 & -1.315 & 0.698 \\
\hline 0 & 1.858 & -1.654 & -0.687 \\
\hline $\mathrm{C}$ & 1.670 & -0.951 & 1.970 \\
\hline $\mathrm{H}$ & 4.067 & -0.112 & 0.788 \\
\hline $\mathrm{H}$ & 3.118 & 1.411 & 0.901 \\
\hline $\mathrm{H}$ & 3.618 & 0.757 & -0.708 \\
\hline $\mathrm{H}$ & -1.628 & -3.399 & 1.234 \\
\hline $\mathrm{H}$ & -0.120 & -2.830 & 0.450 \\
\hline $\mathrm{H}$ & -1.600 & -3.074 & -0.537 \\
\hline $\mathrm{H}$ & -3.796 & -2.031 & 1.496 \\
\hline $\mathrm{H}$ & -3.814 & -1.752 & -0.275 \\
\hline $\mathrm{H}$ & -4.067 & -0.374 & 0.885 \\
\hline $\mathrm{H}$ & 2.607 & -1.472 & 2.251 \\
\hline $\mathrm{H}$ & 0.836 & -1.648 & 2.172 \\
\hline $\mathrm{H}$ & 1.564 & -0.060 & 2.620 \\
\hline $\mathrm{C}$ & -2.104 & 2.153 & -0.424 \\
\hline $\mathrm{C}$ & -1.194 & 2.513 & 0.492 \\
\hline $\mathrm{C}$ & 0.088 & 1.763 & 0.753 \\
\hline $\mathrm{C}$ & 0.364 & 0.660 & -0.303 \\
\hline $\mathrm{C}$ & -0.982 & -0.094 & -0.665 \\
\hline $\mathrm{C}$ & -1.930 & 0.953 & -1.329 \\
\hline $\mathrm{C}$ & 0.922 & 1.361 & -1.580 \\
\hline $\mathrm{C}$ & -3.280 & 0.429 & -1.851 \\
\hline $\mathrm{H}$ & -3.015 & 2.761 & -0.554 \\
\hline $\mathrm{H}$ & -1.385 & 3.399 & 1.121 \\
\hline $\mathrm{H}$ & 0.043 & 1.347 & 1.781 \\
\hline $\mathrm{H}$ & 0.929 & 2.492 & 0.760 \\
\hline $\mathrm{H}$ & -0.748 & -0.843 & -1.450 \\
\hline $\mathrm{H}$ & -1.426 & 1.325 & -2.247 \\
\hline $\mathrm{H}$ & 1.026 & 0.656 & -2.435 \\
\hline $\mathrm{H}$ & 1.919 & 1.821 & -1.416 \\
\hline
\end{tabular}




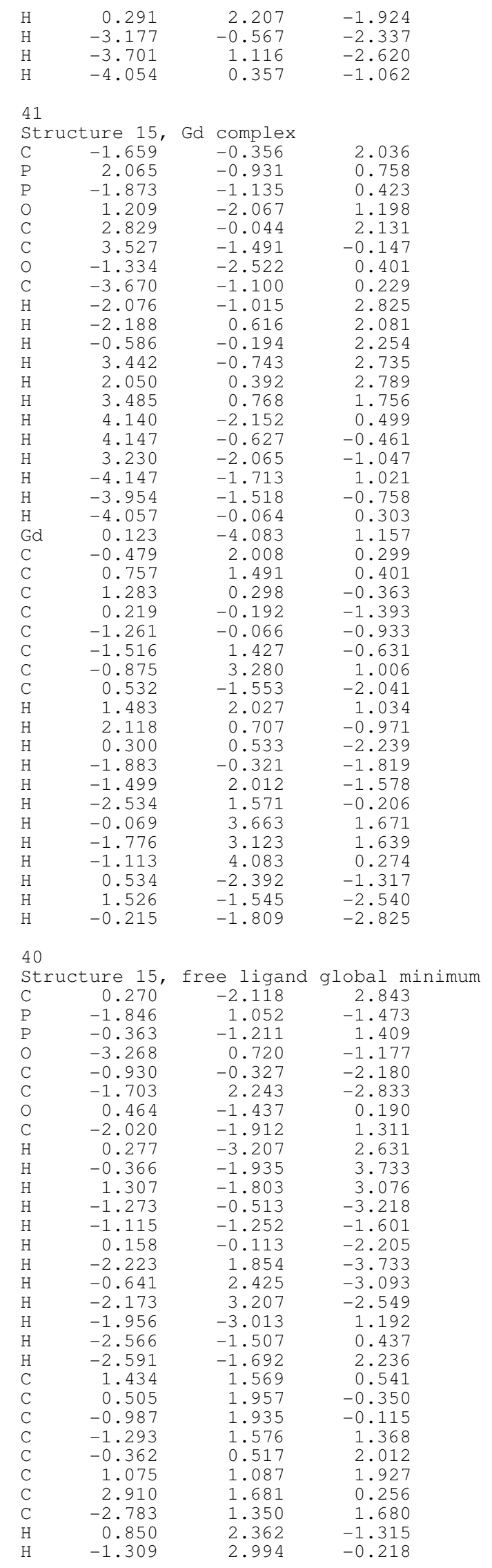




\begin{tabular}{|c|c|c|c|}
\hline $\mathrm{H}$ & -1.052 & 2.513 & 1.928 \\
\hline $\mathrm{H}$ & -0.632 & 0.471 & 3.089 \\
\hline $\mathrm{H}$ & 1.180 & 1.946 & 2.630 \\
\hline $\mathrm{H}$ & 1.815 & 0.328 & 2.266 \\
\hline $\mathrm{H}$ & 3.115 & 2.063 & -0.769 \\
\hline $\mathrm{H}$ & 3.409 & 0.691 & 0.344 \\
\hline $\mathrm{H}$ & 3.402 & 2.375 & 0.973 \\
\hline $\mathrm{H}$ & -3.186 & 0.419 & 1.237 \\
\hline $\mathrm{H}$ & -3.409 & 2.191 & 1.304 \\
\hline $\mathrm{H}$ & -2.962 & 1.284 & 2.776 \\
\hline \multicolumn{4}{|c|}{41} \\
\hline \multicolumn{4}{|c|}{ Structure 16, Gd complex } \\
\hline $\mathrm{P}$ & -1.777 & -0.355 & 0.020 \\
\hline 0 & -1.639 & -1.782 & 0.419 \\
\hline $\mathrm{C}$ & -3.413 & 0.004 & -0.661 \\
\hline $\mathrm{C}$ & -1.712 & 0.746 & 1.448 \\
\hline $\mathrm{P}$ & 1.911 & -0.937 & 1.370 \\
\hline 0 & 0.698 & -1.695 & 1.785 \\
\hline $\mathrm{C}$ & 2.599 & 0.080 & 2.691 \\
\hline $\mathrm{C}$ & 3.308 & -2.028 & 1.014 \\
\hline $\mathrm{H}$ & -4.196 & -0.273 & 0.073 \\
\hline $\mathrm{H}$ & -3.576 & -0.584 & -1.588 \\
\hline $\mathrm{H}$ & -3.518 & 1.081 & -0.900 \\
\hline $\mathrm{H}$ & -2.457 & 0.419 & 2.203 \\
\hline $\mathrm{H}$ & -1.947 & 1.790 & 1.160 \\
\hline $\mathrm{H}$ & -0.710 & 0.717 & 1.919 \\
\hline $\mathrm{H}$ & 2.861 & -0.558 & 3.560 \\
\hline $\mathrm{H}$ & 1.855 & 0.833 & 3.025 \\
\hline $\mathrm{H}$ & 3.515 & 0.602 & 2.347 \\
\hline $\mathrm{H}$ & 3.566 & -2.616 & 1.918 \\
\hline $\mathrm{H}$ & 4.196 & -1.439 & 0.708 \\
\hline $\mathrm{H}$ & 3.050 & -2.737 & 0.202 \\
\hline $\mathrm{Gd}$ & -0.874 & -3.359 & 1.854 \\
\hline $\mathrm{C}$ & 0.420 & 2.250 & -0.328 \\
\hline $\mathrm{C}$ & 1.417 & 1.533 & 0.206 \\
\hline $\mathrm{C}$ & 1.751 & 0.101 & -0.138 \\
\hline $\mathrm{C}$ & 0.830 & -0.395 & -1.297 \\
\hline $\mathrm{C}$ & -0.617 & 0.181 & -1.300 \\
\hline $\mathrm{C}$ & -0.537 & 1.730 & -1.372 \\
\hline $\mathrm{C}$ & 0.872 & -1.914 & -1.545 \\
\hline $\mathrm{C}$ & -0.122 & 2.248 & -2.762 \\
\hline $\mathrm{H}$ & 0.299 & 3.306 & -0.031 \\
\hline $\mathrm{H}$ & 2.092 & 2.047 & 0.911 \\
\hline $\mathrm{H}$ & 2.774 & 0.150 & -0.568 \\
\hline $\mathrm{H}$ & 1.295 & 0.029 & -2.217 \\
\hline $\mathrm{H}$ & -1.081 & -0.158 & -2.251 \\
\hline $\mathrm{H}$ & -1.543 & 2.158 & -1.157 \\
\hline $\mathrm{H}$ & 0.257 & -2.197 & -2.429 \\
\hline $\mathrm{H}$ & 0.494 & -2.510 & -0.691 \\
\hline $\mathrm{H}$ & 1.907 & -2.262 & -1.757 \\
\hline $\mathrm{H}$ & 0.918 & 1.962 & -3.034 \\
\hline $\mathrm{H}$ & -0.794 & 1.861 & -3.560 \\
\hline $\mathrm{H}$ & -0.171 & 3.360 & -2.809 \\
\hline \multicolumn{4}{|c|}{40} \\
\hline \multicolumn{4}{|c|}{ Str } \\
\hline $\mathrm{P}$ & 1.855 & -0.080 & 1.052 \\
\hline 0 & 1.715 & -1.465 & 0.523 \\
\hline $\mathrm{C}$ & 1.318 & 0.108 & 2.762 \\
\hline $\mathrm{C}$ & 3.591 & 0.414 & 1.228 \\
\hline $\mathrm{P}$ & -2.020 & -0.766 & 0.572 \\
\hline 0 & -2.421 & -0.177 & 1.882 \\
\hline $\mathrm{C}$ & -1.124 & $-2 \cdot 318$ & 0.740 \\
\hline $\mathrm{C}$ & -3.457 & -1.340 & -0.373 \\
\hline $\mathrm{H}$ & 1.939 & -0.530 & 3.423 \\
\hline $\mathrm{H}$ & 0.264 & -0.210 & 2.878 \\
\hline $\mathrm{H}$ & 1.425 & 1.162 & 3.091 \\
\hline $\mathrm{H}$ & 4.112 & -0.275 & 1.923 \\
\hline $\mathrm{H}$ & 3.663 & 1.445 & 1.631 \\
\hline $\mathrm{H}$ & 4.107 & 0.377 & 0.248 \\
\hline $\mathrm{H}$ & -1.817 & -3.118 & 1.072 \\
\hline $\mathrm{H}$ & -0.333 & -2.227 & 1.508 \\
\hline $\mathrm{H}$ & -0.674 & -2.625 & -0.226 \\
\hline $\mathrm{H}$ & -4.046 & -2.059 & 0.233 \\
\hline $\mathrm{H}$ & -3.142 & -1.845 & -1.308 \\
\hline $\mathrm{H}$ & -4.112 & -0.483 & -0.631 \\
\hline $\mathrm{C}$ & 0.642 & -0.085 & -2.157 \\
\hline $\mathrm{C}$ & -0.622 & -0.272 & -1.752 \\
\hline $\mathrm{C}$ & -1.267 & 0.438 & -0.587 \\
\hline $\mathrm{C}$ & -0.318 & 1.551 & -0.052 \\
\hline
\end{tabular}




\begin{tabular}{|c|c|c|c|}
\hline $\mathrm{C}$ & 1.198 & 1.219 & -0.063 \\
\hline $\mathrm{C}$ & 1.612 & 0.887 & -1.528 \\
\hline $\mathrm{C}$ & -0.790 & 2.220 & 1.251 \\
\hline $\mathrm{C}$ & 1.733 & 2.142 & -2.412 \\
\hline $\mathrm{H}$ & 1.010 & -0.647 & -3.032 \\
\hline $\mathrm{H}$ & -1.250 & -0.974 & -2.327 \\
\hline $\mathrm{H}$ & -2.128 & 0.988 & -1.024 \\
\hline $\mathrm{H}$ & -0.418 & 2.370 & -0.803 \\
\hline $\mathrm{H}$ & 1.725 & 2.155 & 0.220 \\
\hline $\mathrm{H}$ & 2.615 & 0.400 & -1.522 \\
\hline $\mathrm{H}$ & -0.177 & 3.118 & 1.490 \\
\hline $\mathrm{H}$ & -0.733 & 1.553 & 2.132 \\
\hline $\mathrm{H}$ & -1.845 & 2.567 & 1.171 \\
\hline $\mathrm{H}$ & 0.758 & 2.655 & -2.565 \\
\hline $\mathrm{H}$ & 2.434 & 2.886 & -1.972 \\
\hline $\mathrm{H}$ & 2.125 & 1.889 & -3.423 \\
\hline \multicolumn{4}{|c|}{41} \\
\hline \multicolumn{4}{|c|}{ Structure 17, Gd comple } \\
\hline $\mathrm{P}$ & -1.869 & -1.181 & 0.235 \\
\hline 0 & -1.216 & -2.178 & 1.127 \\
\hline $\mathrm{C}$ & -2.684 & -1.925 & -1.195 \\
\hline $\mathrm{C}$ & -3.312 & -0.387 & 0.987 \\
\hline $\mathrm{P}$ & 1.998 & -0.823 & -0.104 \\
\hline 0 & 1.599 & -1.826 & 0.924 \\
\hline $\mathrm{C}$ & 3.089 & 0.460 & 0.558 \\
\hline $\mathrm{C}$ & 3.157 & -1.535 & -1.302 \\
\hline $\mathrm{H}$ & -3.557 & -2.523 & -0.862 \\
\hline $\mathrm{H}$ & -1.999 & -2.607 & -1.734 \\
\hline $\mathrm{H}$ & -3.041 & -1.140 & -1.893 \\
\hline $\mathrm{H}$ & -4.088 & -1.147 & 1.214 \\
\hline $\mathrm{H}$ & -3.744 & 0.366 & 0.296 \\
\hline $\mathrm{H}$ & -3.037 & 0.114 & 1.936 \\
\hline $\mathrm{H}$ & 3.947 & -0.014 & 1.078 \\
\hline $\mathrm{H}$ & 2.568 & 1.106 & 1.288 \\
\hline $\mathrm{H}$ & 3.492 & 1.096 & -0.257 \\
\hline $\mathrm{H}$ & 4.088 & -1.847 & -0.785 \\
\hline $\mathrm{H}$ & 3.423 & -0.799 & -2.087 \\
\hline $\mathrm{H}$ & 2.718 & -2.434 & -1.780 \\
\hline $\mathrm{Gd}$ & 0.430 & -3.237 & 2.271 \\
\hline $\mathrm{C}$ & 0.359 & 2.353 & 0.374 \\
\hline $\mathrm{C}$ & 1.050 & 2.332 & -0.773 \\
\hline $\mathrm{C}$ & 0.994 & 1.185 & -1.749 \\
\hline $\mathrm{C}$ & 0.559 & -0.144 & -1.071 \\
\hline $\mathrm{C}$ & -0.765 & 0.198 & -0.291 \\
\hline $\mathrm{C}$ & -0.563 & 1.252 & 0.843 \\
\hline $\mathrm{C}$ & 0.273 & -1.149 & -2.213 \\
\hline $\mathrm{C}$ & -0.121 & 0.731 & 2.224 \\
\hline $\mathrm{H}$ & 0.442 & 3.237 & 1.030 \\
\hline $\mathrm{H}$ & 1.667 & 3.203 & -1.054 \\
\hline $\mathrm{H}$ & 0.280 & 1.456 & -2.560 \\
\hline $\mathrm{H}$ & 1.984 & 1.068 & -2.243 \\
\hline $\mathrm{H}$ & -1.381 & 0.733 & -1.046 \\
\hline $\mathrm{H}$ & -1.549 & 1.750 & 1.000 \\
\hline $\mathrm{H}$ & 0.132 & -2.187 & -1.839 \\
\hline $\mathrm{H}$ & -0.637 & -0.869 & -2.788 \\
\hline $\mathrm{H}$ & 1.098 & -1.185 & -2.959 \\
\hline $\mathrm{H}$ & 0.862 & 0.222 & 2.212 \\
\hline $\mathrm{H}$ & -0.845 & 0.010 & 2.660 \\
\hline $\mathrm{H}$ & -0.036 & 1.563 & 2.959 \\
\hline \multicolumn{4}{|c|}{40} \\
\hline \multicolumn{4}{|c|}{ Stru } \\
\hline $\mathrm{P}$ & 1.860 & -0.895 & -0.068 \\
\hline 0 & 1.353 & -2.190 & -0.598 \\
\hline $\mathrm{C}$ & 2.860 & -1.078 & 1.428 \\
\hline $\mathrm{C}$ & 3.110 & -0.125 & -1.129 \\
\hline $\mathrm{P}$ & -2.076 & -0.917 & 0.420 \\
\hline 0 & -2.898 & -0.154 & -0.563 \\
\hline $\mathrm{C}$ & -3.092 & -1.469 & 1.817 \\
\hline $\mathrm{C}$ & -1.555 & -2.526 & -0.205 \\
\hline $\mathrm{H}$ & 3.805 & -1.606 & 1.188 \\
\hline $\mathrm{H}$ & 2.327 & -1.676 & 2.192 \\
\hline $\mathrm{H}$ & 3.111 & -0.085 & 1.854 \\
\hline $\mathrm{H}$ & 3.990 & -0.793 & -1.224 \\
\hline $\mathrm{H}$ & 3.444 & 0.840 & -0.697 \\
\hline $\mathrm{H}$ & 2.708 & 0.057 & -2.145 \\
\hline $\mathrm{H}$ & -3.990 & -2.000 & 1.438 \\
\hline $\mathrm{H}$ & -3.433 & -0.604 & 2.419 \\
\hline $\mathrm{H}$ & -2.531 & -2.168 & 2.469 \\
\hline $\mathrm{H}$ & -2.445 & -3.158 & -0.402 \\
\hline
\end{tabular}




$\begin{array}{rrrr}\mathrm{H} & -0.913 & -3.048 & 0.533 \\ \mathrm{H} & -1.007 & -2.411 & -1.160 \\ \mathrm{C} & -0.847 & 2.210 & -0.801 \\ \mathrm{C} & -1.479 & 2.342 & 0.373 \\ \mathrm{C} & -1.227 & 1.438 & 1.553 \\ \mathrm{C} & -0.652 & 0.051 & 1.132 \\ \mathrm{C} & 0.575 & 0.394 & 0.210 \\ \mathrm{C} & 0.188 & 1.152 & -1.099 \\ \mathrm{C} & -0.196 & -0.648 & 2.434 \\ \mathrm{C} & -0.247 & 0.306 & -2.311 \\ \mathrm{H} & -1.088 & 2.912 & -1.618 \\ \mathrm{H} & -2.209 & 3.158 & 0.510 \\ \mathrm{H} & -0.522 & 1.956 & 2.242 \\ \mathrm{H} & -2.172 & 1.313 & 2.127 \\ \mathrm{H} & 1.129 & 1.159 & 0.796 \\ \mathrm{H} & 1.094 & 1.717 & -1.423 \\ \mathrm{H} & 0.091 & -1.710 & 2.271 \\ \mathrm{H} & 0.675 & -0.131 & 2.892 \\ \mathrm{H} & -0.987 & -0.644 & 3.216 \\ \mathrm{H} & -1.211 & -0.216 & -2.163 \\ \mathrm{H} & 0.501 & -0.470 & -2.583 \\ \mathrm{H} & -0.374 & 0.943 & -3.216\end{array}$

41

\begin{tabular}{|c|c|c|c|}
\hline \multicolumn{4}{|c|}{ Gd complex } \\
\hline $\mathrm{C}$ & -3.491 & -0.527 & 0.859 \\
\hline $\mathrm{P}$ & 1.729 & -0.963 & 0.227 \\
\hline $\mathrm{P}$ & -1.956 & -1.333 & 0.353 \\
\hline 0 & 1.451 & -1.860 & 1.382 \\
\hline $\mathrm{C}$ & 3.293 & -0.075 & 0.376 \\
\hline $\mathrm{C}$ & 1.932 & -1.853 & -1.328 \\
\hline O & -1.255 & -1.973 & 1.502 \\
\hline $\mathrm{C}$ & -2.533 & -2.568 & -0.834 \\
\hline $\mathrm{H}$ & -4.058 & -1.200 & 1.534 \\
\hline $\mathrm{H}$ & -4.136 & -0.300 & -0.012 \\
\hline $\mathrm{H}$ & -3.287 & 0.416 & 1.401 \\
\hline $\mathrm{H}$ & 4.136 & -0.795 & 0.403 \\
\hline $\mathrm{H}$ & 3.312 & 0.519 & 1.312 \\
\hline $\mathrm{H}$ & 3.436 & 0.608 & -0.487 \\
\hline $\mathrm{H}$ & 2.802 & -2.538 & -1.262 \\
\hline $\mathrm{H}$ & 2.100 & -1.143 & -2.164 \\
\hline $\mathrm{H}$ & 1.028 & -2.457 & -1.545 \\
\hline $\mathrm{H}$ & -3.227 & -3.274 & -0.335 \\
\hline $\mathrm{H}$ & -1.671 & -3.143 & -1.229 \\
\hline $\mathrm{H}$ & -3.062 & -2.093 & -1.684 \\
\hline Gd & 0.217 & -3.112 & 2.816 \\
\hline $\mathrm{C}$ & -1.727 & 1.001 & -1.082 \\
\hline $\mathrm{C}$ & -1.553 & 2.211 & -0.519 \\
\hline $\mathrm{C}$ & -0.492 & 2.567 & 0.486 \\
\hline C & 0.308 & 1.363 & 1.035 \\
\hline $\mathrm{C}$ & 0.455 & 0.318 & -0.101 \\
\hline $\mathrm{C}$ & -0.906 & -0.205 & -0.659 \\
\hline $\mathrm{C}$ & -2.701 & 0.824 & -2.224 \\
\hline $\mathrm{C}$ & -0.284 & 0.875 & 2.366 \\
\hline $\mathrm{H}$ & -2.166 & 3.055 & -0.877 \\
\hline $\mathrm{H}$ & -0.954 & 3.132 & 1.328 \\
\hline $\mathrm{H}$ & 0.214 & 3.274 & -0.008 \\
\hline $\mathrm{H}$ & 1.327 & 1.746 & 1.279 \\
\hline $\mathrm{H}$ & 0.890 & 0.897 & -0.946 \\
\hline $\mathrm{H}$ & -0.662 & -0.749 & -1.595 \\
\hline $\mathrm{H}$ & -2.170 & 0.495 & -3.145 \\
\hline $\mathrm{H}$ & -3.229 & 1.770 & -2.477 \\
\hline $\mathrm{H}$ & -3.484 & 0.070 & -2.007 \\
\hline $\mathrm{H}$ & 0.263 & -0.001 & 2.773 \\
\hline $\mathrm{H}$ & -1.355 & 0.605 & 2.287 \\
\hline $\mathrm{H}$ & -0.220 & 1.668 & 3.145 \\
\hline 40 & & & \\
\hline St & ture 18 , & free ligand & global minimum \\
\hline $\mathrm{C}$ & -1.187 & -1.198 & 2.471 \\
\hline $\mathrm{P}$ & 1.842 & 0.820 & $-1 \cdot 327$ \\
\hline $\mathrm{P}$ & -0.026 & -1.095 & 1.091 \\
\hline 0 & 1.892 & -0.526 & -1.969 \\
\hline $\mathrm{C}$ & 3.211 & 1.128 & -0.198 \\
\hline $\mathrm{C}$ & 2.114 & 2.156 & -2.520 \\
\hline 0 & 1.346 & -0.743 & 1.549 \\
\hline $\mathrm{C}$ & -0.107 & -2.758 & 0.388 \\
\hline $\mathrm{H}$ & -0.715 & -1.747 & 3.311 \\
\hline $\mathrm{H}$ & -2.109 & -1.741 & 2.189 \\
\hline $\mathrm{H}$ & -1.465 & -0.187 & 2.831 \\
\hline $\mathrm{H}$ & 4.172 & 1.019 & -0.741 \\
\hline
\end{tabular}




\begin{tabular}{|c|c|c|c|}
\hline $\mathrm{H}$ & 3.213 & 0.406 & 0.640 \\
\hline $\mathrm{H}$ & 3.161 & 2.159 & 0.208 \\
\hline $\mathrm{H}$ & 3.107 & 2.040 & -3.000 \\
\hline $\mathrm{H}$ & 2.077 & 3.145 & -2.019 \\
\hline $\mathrm{H}$ & 1.337 & 2.128 & -3.311 \\
\hline $\mathrm{H}$ & 0.206 & -3.505 & 1.146 \\
\hline $\mathrm{H}$ & 0.573 & -2.836 & -0.485 \\
\hline $\mathrm{H}$ & -1.138 & -2.998 & 0.058 \\
\hline C & -2.086 & 0.435 & 0.034 \\
\hline C & -2.379 & 1.714 & 0.336 \\
\hline $\mathrm{C}$ & -1.398 & 2.857 & 0.342 \\
\hline $\mathrm{C}$ & 0.078 & 2.399 & 0.323 \\
\hline $\mathrm{C}$ & 0.176 & 1.215 & -0.673 \\
\hline $\mathrm{C}$ & -0.670 & -0.028 & -0.263 \\
\hline $\mathrm{C}$ & -3.199 & -0.567 & -0.173 \\
\hline C & 0.550 & 2.163 & 1.767 \\
\hline $\mathrm{H}$ & -3.434 & 1.995 & 0.489 \\
\hline $\mathrm{H}$ & -1.584 & 3.505 & 1.228 \\
\hline $\mathrm{H}$ & -1.601 & 3.489 & -0.553 \\
\hline $\mathrm{H}$ & 0.684 & 3.245 & -0.078 \\
\hline $\mathrm{H}$ & -0.351 & 1.592 & -1.578 \\
\hline $\mathrm{H}$ & -0.745 & -0.665 & -1.170 \\
\hline $\mathrm{H}$ & -3.343 & -0.769 & -1.257 \\
\hline $\mathrm{H}$ & -4.172 & -0.204 & 0.225 \\
\hline $\mathrm{H}$ & -2.996 & -1.544 & 0.312 \\
\hline $\mathrm{H}$ & 1.580 & 1.755 & 1.821 \\
\hline $\mathrm{H}$ & -0.121 & 1.479 & 2.327 \\
\hline $\mathrm{H}$ & 0.565 & 3.118 & 2.340 \\
\hline \multicolumn{4}{|c|}{41} \\
\hline \multicolumn{4}{|c|}{ Structure 19, Gd complex } \\
\hline $\mathrm{C}$ & -3.258 & -1.332 & -0.497 \\
\hline $\mathrm{P}$ & 1.472 & -2.179 & 0.927 \\
\hline $\mathrm{P}$ & -1.535 & -1.086 & -0.986 \\
\hline O & 1.409 & -3.134 & -0.213 \\
\hline C & 0.585 & -2.739 & 2.392 \\
\hline $\mathrm{C}$ & 3.148 & $-1 \cdot 912$ & 1.538 \\
\hline O & -0.917 & -2.383 & -1.385 \\
\hline $\mathrm{C}$ & -1.676 & 0.022 & -2.402 \\
\hline $\mathrm{H}$ & -3.783 & -1.926 & -1.273 \\
\hline $\mathrm{H}$ & -3.794 & -0.372 & -0.380 \\
\hline $\mathrm{H}$ & -3.305 & -1.890 & 0.460 \\
\hline $\mathrm{H}$ & 1.046 & -3.670 & 2.780 \\
\hline $\mathrm{H}$ & -0.474 & -2.951 & 2.140 \\
\hline $\mathrm{H}$ & 0.620 & -1.965 & 3.185 \\
\hline $\mathrm{H}$ & 3.577 & -2.870 & 1.897 \\
\hline $\mathrm{H}$ & 3.145 & -1.187 & 2.378 \\
\hline $\mathrm{H}$ & 3.794 & -1.519 & 0.727 \\
\hline $\mathrm{H}$ & -2.299 & -0.456 & -3.185 \\
\hline $\mathrm{H}$ & -0.678 & 0.224 & -2.840 \\
\hline $\mathrm{H}$ & -2.149 & 0.983 & -2.125 \\
\hline $\mathrm{Gd}$ & 0.510 & -4.017 & -2.094 \\
\hline $\mathrm{C}$ & -0.140 & 1.987 & -0.417 \\
\hline $\mathrm{C}$ & 0.930 & 1.447 & -1.033 \\
\hline $\mathrm{C}$ & 1.479 & 0.071 & -0.749 \\
\hline $\mathrm{C}$ & 0.846 & -0.505 & 0.532 \\
\hline C & -0.702 & -0.312 & 0.481 \\
\hline C & -0.919 & 1.225 & 0.642 \\
\hline C & -0.538 & 3.418 & -0.682 \\
\hline $\mathrm{C}$ & -2.400 & 1.631 & 0.748 \\
\hline $\mathrm{H}$ & 1.474 & 2.036 & -1.791 \\
\hline $\mathrm{H}$ & 1.304 & -0.592 & -1.625 \\
\hline $\mathrm{H}$ & 2.583 & 0.148 & -0.622 \\
\hline $\mathrm{H}$ & 1.205 & 0.130 & 1.371 \\
\hline $\mathrm{H}$ & -1.129 & -0.793 & 1.386 \\
\hline $\mathrm{H}$ & -0.464 & 1.521 & 1.617 \\
\hline $\mathrm{H}$ & -1.561 & 3.483 & -1.112 \\
\hline $\mathrm{H}$ & -0.520 & 4.017 & 0.255 \\
\hline $\mathrm{H}$ & 0.147 & 3.919 & -1.401 \\
\hline $\mathrm{H}$ & -2.949 & 0.985 & 1.468 \\
\hline $\mathrm{H}$ & -2.510 & 2.674 & 1.121 \\
\hline $\mathrm{H}$ & -2.934 & 1.587 & -0.224 \\
\hline \multicolumn{4}{|l|}{40} \\
\hline \multicolumn{4}{|c|}{ Str } \\
\hline $\mathrm{C}$ & -0.959 & -2.544 & 1.539 \\
\hline $\mathrm{P}$ & 0.526 & 2.165 & 1.291 \\
\hline P & -1.402 & -1.091 & 0.562 \\
\hline O & -0.278 & 2.117 & 2.544 \\
\hline $\mathrm{C}$ & -0.282 & 3.030 & -0.070 \\
\hline $\mathrm{C}$ & 2.063 & 3.094 & 1.480 \\
\hline
\end{tabular}




\begin{tabular}{|c|c|c|c|}
\hline 0 & -2.413 & -1.431 & -0.484 \\
\hline $\mathrm{C}$ & -2.145 & -0.068 & 1.845 \\
\hline $\mathrm{H}$ & -1.879 & -3.010 & 1.948 \\
\hline $\mathrm{H}$ & -0.305 & -2.280 & 2.392 \\
\hline $\mathrm{H}$ & -0.448 & -3.300 & 0.911 \\
\hline $\mathrm{H}$ & -0.468 & 4.087 & 0.211 \\
\hline $\mathrm{H}$ & -1.256 & 2.554 & -0.305 \\
\hline $\mathrm{H}$ & 0.357 & 3.009 & -0.976 \\
\hline $\mathrm{H}$ & 1.841 & 4.138 & 1.781 \\
\hline $\mathrm{H}$ & 2.628 & 3.113 & 0.526 \\
\hline $\mathrm{H}$ & 2.698 & 2.630 & 2.262 \\
\hline $\mathrm{H}$ & -3.085 & -0.539 & 2.198 \\
\hline $\mathrm{H}$ & -2.393 & 0.935 & 1.444 \\
\hline $\mathrm{H}$ & -1.466 & 0.026 & 2.715 \\
\hline $\mathrm{C}$ & 1.801 & -2.107 & -0.058 \\
\hline $\mathrm{C}$ & 2.104 & -1.677 & 1. 182 \\
\hline $\mathrm{C}$ & 1.664 & -0.356 & 1.760 \\
\hline $\mathrm{C}$ & 1.068 & 0.537 & 0.660 \\
\hline $\mathrm{C}$ & 0.064 & -0.268 & -0.212 \\
\hline $\mathrm{C}$ & 0.944 & -1.288 & -1.009 \\
\hline $\mathrm{C}$ & 2.393 & -3.390 & -0.589 \\
\hline $\mathrm{C}$ & 0.129 & -2.144 & -2.000 \\
\hline $\mathrm{H}$ & 2.735 & -2.304 & 1.834 \\
\hline $\mathrm{H}$ & 0.938 & -0.521 & 2.586 \\
\hline $\mathrm{H}$ & 2.550 & 0.145 & 2.214 \\
\hline $\mathrm{H}$ & 1.913 & 0.782 & -0.019 \\
\hline $\mathrm{H}$ & -0.347 & 0.438 & -0.964 \\
\hline $\mathrm{H}$ & 1.658 & -0.700 & -1.633 \\
\hline $\mathrm{H}$ & 1.603 & -4.138 & -0.821 \\
\hline $\mathrm{H}$ & 2.975 & -3.206 & -1.519 \\
\hline $\mathrm{H}$ & 3.085 & -3.868 & 0.139 \\
\hline $\mathrm{H}$ & -0.550 & -1.515 & -2.618 \\
\hline $\mathrm{H}$ & 0.790 & -2.682 & -2.715 \\
\hline $\mathrm{H}$ & -0.493 & -2.916 & -1.497 \\
\hline \multicolumn{4}{|c|}{36} \\
\hline \multicolumn{4}{|c|}{ Structure 20, Gd complex } \\
\hline $\mathrm{P}$ & 1.964 & -0.959 & -0.409 \\
\hline 0 & 1.279 & -1.455 & -1.633 \\
\hline $\mathrm{C}$ & 2.282 & -2.250 & 0.806 \\
\hline $\mathrm{C}$ & 3.595 & -0.270 & -0.751 \\
\hline $\mathrm{P}$ & -1.978 & -1.675 & -0.050 \\
\hline 0 & -1.143 & -2.454 & -1.004 \\
\hline $\mathrm{C}$ & -3.628 & -1.334 & -0.695 \\
\hline $\mathrm{C}$ & -2.296 & -2.517 & 1.510 \\
\hline $\mathrm{H}$ & 2.926 & -3.037 & 0.363 \\
\hline $\mathrm{H}$ & 1.327 & -2.713 & 1.127 \\
\hline $\mathrm{H}$ & 2.792 & -1.827 & 1.695 \\
\hline $\mathrm{H}$ & 4.231 & -1.031 & -1.247 \\
\hline $\mathrm{H}$ & 4.089 & 0.048 & 0.190 \\
\hline $\mathrm{H}$ & 3.504 & 0.608 & -1.422 \\
\hline $\mathrm{H}$ & -4.146 & -2.285 & -0.933 \\
\hline $\mathrm{H}$ & -3.555 & -0.728 & -1.621 \\
\hline $\mathrm{H}$ & -4.231 & -0.776 & 0.051 \\
\hline $\mathrm{H}$ & -2.851 & -3.460 & 1.326 \\
\hline $\mathrm{H}$ & -2.898 & -1.872 & 2.182 \\
\hline $\mathrm{H}$ & -1.339 & -2.764 & 2.013 \\
\hline $\mathrm{Gd}$ & 0.044 & -2.876 & -2.897 \\
\hline $\mathrm{C}$ & 0.464 & 1.492 & -0.301 \\
\hline $\mathrm{C}$ & -0.837 & 0.836 & -0.838 \\
\hline $\mathrm{C}$ & -1.353 & -0.020 & 0.372 \\
\hline $\mathrm{C}$ & -0.151 & 0.084 & 1.367 \\
\hline $\mathrm{C}$ & -0.306 & 1.524 & 1.893 \\
\hline $\mathrm{C}$ & 0.027 & 2.357 & 0.891 \\
\hline $\mathrm{C}$ & 1.121 & 0.352 & 0.517 \\
\hline $\mathrm{H}$ & 1.119 & 2.004 & -1.044 \\
\hline $\mathrm{H}$ & -1.583 & 1.604 & -1.147 \\
\hline $\mathrm{H}$ & -2.210 & 0.517 & 0.835 \\
\hline $\mathrm{H}$ & -0.063 & -0.685 & 2.169 \\
\hline $\mathrm{H}$ & -0.674 & 1.795 & 2.897 \\
\hline $\mathrm{H}$ & 0.001 & 3.460 & 0.895 \\
\hline $\mathrm{H}$ & 1.899 & 0.784 & 1.186 \\
\hline $\mathrm{H}$ & -0.618 & 0.207 & -1.729 \\
\hline \multicolumn{4}{|c|}{35} \\
\hline St & ture 20, & free ligand & global minimum \\
\hline $\mathrm{P}$ & -1.888 & -1.498 & -0.130 \\
\hline 0 & -1.046 & -2.450 & 0.648 \\
\hline $\mathrm{C}$ & -2.078 & -1.901 & -1.877 \\
\hline $\mathrm{C}$ & -3.597 & -1.446 & 0.452 \\
\hline $\mathrm{P}$ & 1.934 & -1.276 & -0.381 \\
\hline
\end{tabular}




$\begin{array}{rrr}1.236 & -1.970 & -1.500 \\ 2.361 & -2.336 & 1.012 \\ 3.555 & -0.642 & -0.870 \\ -2.487 & -2.926 & -1.988 \\ -1.102 & -1.851 & -2.398 \\ -2.772 & -1.186 & -2.363 \\ -4.056 & -2.452 & 0.370 \\ -4.194 & -0.733 & -0.153 \\ -3.629 & -1.128 & 1.513 \\ 3.060 & -3.131 & 0.681 \\ 1.456 & -2.821 & 1.428 \\ 2.851 & -1.744 & 1.812 \\ 4.194 & -1.473 & -1.233 \\ 4.060 & -0.156 & -0.011 \\ 3.443 & 0.100 & -1.687 \\ -1.028 & 0.794 & 1.331 \\ 0.357 & 0.151 & 1.612 \\ 1.094 & 0.222 & 0.227 \\ -0.046 & 0.737 & -0.713 \\ -0.180 & 2.214 & -0.302 \\ -0.738 & 2.245 & 0.921 \\ -1.377 & 0.246 & -0.075 \\ -1.811 & 0.672 & 2.115 \\ 0.911 & 0.714 & 2.398 \\ 1.869 & 1.016 & 0.299 \\ 0.068 & 0.578 & -1.810 \\ 0.168 & 3.071 & -0.902 \\ -0.953 & 3.131 & 1.540 \\ -2.218 & 0.833 & -0.509 \\ 0.238 & -0.890 & 1.978\end{array}$
41 Structure 21, Gd complex

\begin{tabular}{|c|c|c|c|}
\hline P & -2.004 & -0.874 & -0.049 \\
\hline 0 & -1.453 & -1.683 & 1.074 \\
\hline $\mathrm{C}$ & -2.473 & -1.919 & -1.455 \\
\hline $\mathrm{C}$ & -3.630 & -0.197 & 0.351 \\
\hline P & 2.247 & -1.889 & 0.384 \\
\hline 0 & 1.265 & -2.802 & 1.032 \\
\hline C & 3.619 & -1.436 & 1.461 \\
\hline C & 3.044 & -2.583 & -1.074 \\
\hline $\mathrm{H}$ & -3.279 & -2.618 & -1.152 \\
\hline $\mathrm{H}$ & -1.611 & -2.524 & -1.800 \\
\hline $\mathrm{H}$ & -2.840 & -1.305 & -2.302 \\
\hline $\mathrm{H}$ & -4.312 & -1.021 & 0.643 \\
\hline $\mathrm{H}$ & -4.073 & 0.321 & -0.522 \\
\hline $\mathrm{H}$ & -3.570 & 0.505 & 1.205 \\
\hline $\mathrm{H}$ & 4.180 & -2.344 & 1.763 \\
\hline $\mathrm{H}$ & 3.237 & -0.935 & 2.374 \\
\hline $\mathrm{H}$ & 4.312 & -0.746 & 0.937 \\
\hline $\mathrm{H}$ & 3.609 & -3.497 & -0.799 \\
\hline $\mathrm{H}$ & 3.747 & -1.848 & -1.517 \\
\hline $\mathrm{H}$ & 2.284 & -2.854 & -1.835 \\
\hline $\mathrm{Gd}$ & -0.559 & -3.426 & 2.225 \\
\hline C & -0.806 & 0.362 & -0.749 \\
\hline C & -1.578 & 1.378 & -1.623 \\
\hline C & -2.052 & 2.487 & -0.614 \\
\hline $\mathrm{C}$ & -1.253 & 2.246 & 0.706 \\
\hline $\mathrm{C}$ & -0.125 & 1.349 & 0.219 \\
\hline $\mathrm{C}$ & 0.860 & 0.496 & 1.003 \\
\hline $\mathrm{C}$ & 1.547 & -0.297 & -0.150 \\
\hline C & 0.449 & -0.389 & -1.283 \\
\hline $\mathrm{H}$ & -0.891 & 1.830 & -2.374 \\
\hline $\mathrm{H}$ & -2.426 & 0.934 & -2.189 \\
\hline $\mathrm{H}$ & -1.827 & 3.497 & -1.028 \\
\hline $\mathrm{H}$ & -1.864 & 1.736 & 1.482 \\
\hline $\mathrm{H}$ & -0.870 & 3.198 & 1.142 \\
\hline $\mathrm{H}$ & 0.518 & 2.024 & -0.397 \\
\hline $\mathrm{H}$ & 1.587 & 1.119 & 1.573 \\
\hline $\mathrm{H}$ & 0.334 & -0.165 & 1.726 \\
\hline $\mathrm{H}$ & 2.377 & 0.328 & -0.544 \\
\hline $\mathrm{H}$ & 0.234 & -1.442 & -1.565 \\
\hline $\mathrm{H}$ & 0.826 & 0.112 & -2.203 \\
\hline $\mathrm{H}$ & -3.149 & 2.443 & -0.431 \\
\hline 40 & & & \\
\hline & cure 21 , & free 1 & minimu \\
\hline P & -1.998 & -1 . & -0 . \\
\hline O & -1.013 & -2.163 & -0.611 \\
\hline $\mathrm{C}$ & -2.212 & -0.782 & -2.669 \\
\hline $\mathrm{C}$ & -3.684 & -1.601 & -0.501 \\
\hline
\end{tabular}




\begin{tabular}{|c|c|c|c|}
\hline $\mathrm{P}$ & 2.353 & -0.117 & -0.724 \\
\hline 0 & 2.937 & 0.716 & -1.815 \\
\hline C & 1.922 & -1.775 & -1.273 \\
\hline $\mathrm{C}$ & 3.516 & -0.433 & 0.621 \\
\hline $\mathrm{H}$ & -2.658 & -1.674 & -3.153 \\
\hline $\mathrm{H}$ & -1.236 & -0.585 & -3.157 \\
\hline $\mathrm{H}$ & -2.880 & 0.085 & -2.844 \\
\hline $\mathrm{H}$ & -3.928 & -2.525 & -1.063 \\
\hline $\mathrm{H}$ & -4.421 & -0.824 & -0.786 \\
\hline $\mathrm{H}$ & -3.789 & -1.828 & 0.577 \\
\hline $\mathrm{H}$ & 2.815 & -2.270 & -1.706 \\
\hline $\mathrm{H}$ & 1.138 & -1.727 & -2.055 \\
\hline $\mathrm{H}$ & 1.562 & -2.389 & -0.423 \\
\hline $\mathrm{H}$ & 4.421 & -0.939 & 0.229 \\
\hline $\mathrm{H}$ & 3.053 & -1.081 & 1.392 \\
\hline $\mathrm{H}$ & 3.820 & 0.524 & 1.093 \\
\hline $\mathrm{C}$ & -1.534 & 0.567 & -0.173 \\
\hline $\mathrm{C}$ & -2.798 & 1.449 & -0.057 \\
\hline $\mathrm{C}$ & -3.426 & 1.057 & 1.330 \\
\hline C & -2.336 & 0.229 & 2.085 \\
\hline $\mathrm{C}$ & -1.084 & 0.592 & 1.302 \\
\hline C & 0.297 & -0.051 & 1.287 \\
\hline C & 0.959 & 0.709 & 0.101 \\
\hline $\mathrm{C}$ & -0.238 & 1.093 & -0.855 \\
\hline $\mathrm{H}$ & -2.509 & 2.525 & -0.040 \\
\hline $\mathrm{H}$ & -3.513 & 1.322 & -0.900 \\
\hline $\mathrm{H}$ & -3.683 & 1.978 & 1.904 \\
\hline $\mathrm{H}$ & -2.534 & -0.864 & 2.043 \\
\hline $\mathrm{H}$ & -2.257 & 0.522 & 3.157 \\
\hline $\mathrm{H}$ & -0.891 & 1.659 & 1.569 \\
\hline $\mathrm{H}$ & 0.839 & 0.117 & 2.245 \\
\hline $\mathrm{H}$ & 0.235 & -1.147 & 1.118 \\
\hline $\mathrm{H}$ & 1.353 & 1.663 & 0.512 \\
\hline $\mathrm{H}$ & -0.095 & 0.691 & -1.880 \\
\hline $\mathrm{H}$ & -0.288 & 2.201 & -0.958 \\
\hline $\mathrm{H}$ & -4.365 & 0.471 & 1.212 \\
\hline \multicolumn{4}{|l|}{45} \\
\hline Str & ture 22, & complex & \\
\hline $\mathrm{P}$ & 1.990 & -1.245 & -0.128 \\
\hline O & 1.375 & -1.688 & -1.408 \\
\hline $\mathrm{C}$ & 2.107 & -2.536 & 1.124 \\
\hline $\mathrm{C}$ & 3.729 & -0.789 & -0.324 \\
\hline $\mathrm{P}$ & -2.038 & -1.425 & -0.135 \\
\hline O & -1.382 & -1.811 & -1.413 \\
\hline C & -3.810 & -1.126 & -0.337 \\
\hline C & -2.044 & -2.721 & 1.117 \\
\hline $\mathrm{H}$ & 2.724 & -3.376 & 0.746 \\
\hline $\mathrm{H}$ & 1.102 & -2.926 & 1.372 \\
\hline $\mathrm{H}$ & 2.573 & -2.138 & 2.049 \\
\hline $\mathrm{H}$ & 4.309 & -1.661 & -0.691 \\
\hline $\mathrm{H}$ & 4.159 & -0.459 & 0.643 \\
\hline $\mathrm{H}$ & 3.832 & 0.033 & -1.061 \\
\hline $\mathrm{H}$ & -4.309 & -2.046 & -0.706 \\
\hline $\mathrm{H}$ & -3.983 & -0.316 & -1.074 \\
\hline $\mathrm{H}$ & -4.272 & -0.836 & 0.629 \\
\hline $\mathrm{H}$ & -2.582 & -3.613 & 0.737 \\
\hline $\mathrm{H}$ & -2.547 & -2.367 & 2.040 \\
\hline $\mathrm{H}$ & -1.009 & -3.020 & 1.369 \\
\hline $\mathrm{Gd}$ & 0.033 & -2.501 & -3.045 \\
\hline $\mathrm{C}$ & -0.090 & 0.078 & 1.457 \\
\hline $\mathrm{C}$ & 1.242 & 0.217 & 0.666 \\
\hline C & 1.124 & 1.439 & -0.274 \\
\hline $\mathrm{C}$ & 1.040 & 2.702 & 0.608 \\
\hline $\mathrm{C}$ & -0.204 & 2.623 & 1.512 \\
\hline C & -1.447 & 2.591 & 0.604 \\
\hline $\mathrm{C}$ & -1.414 & 1.326 & -0.278 \\
\hline C & -1.426 & 0.097 & 0.661 \\
\hline $\mathrm{C}$ & -0.142 & 1.351 & -1.146 \\
\hline $\mathrm{C}$ & -0.148 & 1.337 & 2.370 \\
\hline $\mathrm{H}$ & 2.000 & 0.494 & 1.428 \\
\hline $\mathrm{H}$ & 2.023 & 1.519 & -0.928 \\
\hline $\mathrm{H}$ & 1.959 & 2.803 & 1.229 \\
\hline $\mathrm{H}$ & 0.993 & 3.613 & -0.032 \\
\hline $\mathrm{H}$ & -0.245 & 3.519 & 2.175 \\
\hline $\mathrm{H}$ & -1.479 & 3.503 & -0.036 \\
\hline $\mathrm{H}$ & -2.374 & 2.609 & 1.222 \\
\hline $\mathrm{H}$ & -2.315 & 1.325 & -0.936 \\
\hline $\mathrm{H}$ & -2.209 & 0.306 & 1.421 \\
\hline $\mathrm{H}$ & -0.101 & 0.452 & -1.799 \\
\hline $\mathrm{H}$ & -0.180 & 2.226 & -1.834 \\
\hline
\end{tabular}




\begin{tabular}{|c|c|c|c|}
\hline $\begin{array}{l}\mathrm{H} \\
\mathrm{H} \\
\mathrm{H}\end{array}$ & $\begin{array}{r}-1.036 \\
0.738 \\
-0.051\end{array}$ & $\begin{array}{r}1.290 \\
1.370 \\
-0.825\end{array}$ & $\begin{array}{l}3.042 \\
3.045 \\
2.110\end{array}$ \\
\hline \multicolumn{4}{|l|}{44} \\
\hline \multicolumn{4}{|c|}{ Structure 22, } \\
\hline $\mathrm{P}$ & 1.902 & -1.923 & 0.542 \\
\hline O & 1.234 & -2.844 & 1.505 \\
\hline $\mathrm{C}$ & 3.670 & -1.732 & 0.901 \\
\hline C & 2.035 & -2.567 & -1.138 \\
\hline $\mathrm{P}$ & -1.924 & -1.761 & 0.059 \\
\hline 0 & -1.151 & -2.543 & -0.943 \\
\hline $\mathrm{C}$ & -3.640 & -1.493 & -0.445 \\
\hline $\mathrm{C}$ & -2.150 & -2.583 & 1.649 \\
\hline $\mathrm{H}$ & 4.183 & -2.712 & 0.820 \\
\hline $\mathrm{H}$ & 3.812 & -1.348 & 1.931 \\
\hline $\mathrm{H}$ & 4.140 & -1.024 & 0.187 \\
\hline $\mathrm{H}$ & 2.578 & -3.534 & -1.126 \\
\hline $\mathrm{H}$ & 2.595 & -1.864 & -1.786 \\
\hline $\mathrm{H}$ & 1.041 & -2.745 & -1.587 \\
\hline $\mathrm{H}$ & -4.156 & -2.467 & -0.566 \\
\hline $\mathrm{H}$ & -3.678 & -0.954 & -1.414 \\
\hline $\mathrm{H}$ & -4.183 & -0.896 & 0.317 \\
\hline $\mathrm{H}$ & -2.673 & -3.550 & 1.504 \\
\hline $\mathrm{H}$ & -2.756 & -1.950 & 2.328 \\
\hline $\mathrm{H}$ & -1.174 & -2.783 & 2.132 \\
\hline $\mathrm{C}$ & -0.055 & 0.073 & 1.316 \\
\hline $\mathrm{C}$ & 1.331 & -0.192 & 0.666 \\
\hline $\mathrm{C}$ & 1.443 & 0.656 & -0.621 \\
\hline $\mathrm{C}$ & 1.444 & 2.142 & -0.207 \\
\hline $\mathrm{C}$ & 0.133 & 2.483 & 0.526 \\
\hline $\mathrm{C}$ & -1.037 & 2.255 & -0.448 \\
\hline $\mathrm{C}$ & -1.084 & 0.772 & -0.867 \\
\hline $\mathrm{C}$ & -1.304 & -0.080 & 0.406 \\
\hline $\mathrm{C}$ & 0.245 & 0.411 & -1.558 \\
\hline $\mathrm{C}$ & -0.034 & 1.563 & 1.755 \\
\hline $\mathrm{H}$ & 2.059 & 0.254 & 1.375 \\
\hline $\mathrm{H}$ & 2.397 & 0.431 & -1.154 \\
\hline $\mathrm{H}$ & 2.317 & 2.361 & 0.451 \\
\hline $\mathrm{H}$ & 1.560 & 2.790 & -1.106 \\
\hline $\mathrm{H}$ & 0.149 & 3.551 & 0.851 \\
\hline $\mathrm{H}$ & -0.921 & 2.904 & -1.346 \\
\hline $\mathrm{H}$ & -1.999 & 2.552 & 0.031 \\
\hline $\mathrm{H}$ & -1.928 & 0.629 & -1.582 \\
\hline $\mathrm{H}$ & -2.138 & 0.399 & 0.961 \\
\hline $\mathrm{H}$ & 0.227 & -0.644 & -1.902 \\
\hline $\mathrm{H}$ & 0.363 & 1.027 & -2.480 \\
\hline $\mathrm{H}$ & -0.973 & 1.821 & 2.297 \\
\hline $\mathrm{H}$ & 0.793 & 1.743 & 2.480 \\
\hline $\mathrm{H}$ & -0.171 & -0.555 & 2.230 \\
\hline 34 & & & \\
\hline Str & Eure 23, & Gd complex & \\
\hline $\mathrm{C}$ & 0.416 & 1.344 & -0.935 \\
\hline $\mathrm{C}$ & -0.936 & 1.769 & -0.348 \\
\hline $\mathrm{C}$ & -1.333 & 0.625 & 0.612 \\
\hline $\mathrm{C}$ & 0.040 & 0.113 & 1.155 \\
\hline $\mathrm{C}$ & 1.169 & 0.716 & 0.259 \\
\hline $\mathrm{P}$ & -2.272 & -0.708 & -0.213 \\
\hline $\mathrm{P}$ & 2.390 & -0.521 & -0.281 \\
\hline 0 & -1.495 & -1.453 & -1.243 \\
\hline $\mathrm{C}$ & -3.794 & 0.031 & -0.832 \\
\hline $\mathrm{C}$ & -2.844 & -1.753 & 1.142 \\
\hline 0 & 1.779 & -1.624 & -1.075 \\
\hline $\mathrm{C}$ & 3.220 & -1.069 & 1. 221 \\
\hline $\mathrm{C}$ & 3.646 & 0.410 & -1.174 \\
\hline $\mathrm{Gd}$ & 0.139 & -2.713 & -2.208 \\
\hline $\mathrm{H}$ & 0.968 & 2.210 & -1.368 \\
\hline $\mathrm{H}$ & 0.253 & 0.603 & -1.749 \\
\hline $\mathrm{H}$ & -0.807 & 2.713 & 0.231 \\
\hline $\mathrm{H}$ & -1.694 & 1.967 & -1.139 \\
\hline $\mathrm{H}$ & -1.943 & 1.035 & 1.446 \\
\hline $\mathrm{H}$ & 0.181 & 0.450 & 2.208 \\
\hline $\mathrm{H}$ & 0.078 & -0.999 & 1.172 \\
\hline $\mathrm{H}$ & 1.678 & 1.525 & 0.826 \\
\hline $\mathrm{H}$ & -3.578 & 0.704 & -1.686 \\
\hline $\mathrm{H}$ & -4.293 & 0.615 & -0.032 \\
\hline $\mathrm{H}$ & -4.487 & -0.763 & -1.179 \\
\hline $\mathrm{H}$ & -1.982 & -2.234 & 1.646 \\
\hline $\mathrm{H}$ & -3.513 & -2.547 & 0.751 \\
\hline $\mathrm{H}$ & -3.403 & -1.150 & 1.886 \\
\hline
\end{tabular}




\begin{tabular}{|c|c|c|c|}
\hline \\
\hline $\mathrm{H}$ & 2.507 & -1.609 & 1.877 \\
\hline $\mathrm{H}$ & 3.627 & -0.198 & 1.776 \\
\hline $\mathrm{H}$ & 4.054 & -1.753 & 0.966 \\
\hline $\mathrm{H}$ & 3.214 & 0.842 & -2.100 \\
\hline $\mathrm{H}$ & 4.487 & -0.257 & -1.453 \\
\hline $\mathrm{H}$ & 4.038 & 1.234 & -0.543 \\
\hline \multicolumn{4}{|c|}{33} \\
\hline \multicolumn{4}{|c|}{ ee ligand global mi } \\
\hline $\mathrm{C}$ & -0.974 & -1.410 & -1.194 \\
\hline $\mathrm{C}$ & 0.458 & -0.858 & -1.215 \\
\hline $\mathrm{C}$ & 0.919 & -0.977 & 0.251 \\
\hline $\mathrm{C}$ & -0.379 & -0.756 & 1.086 \\
\hline $\mathrm{C}$ & -1.595 & -0.854 & 0.110 \\
\hline $\mathrm{P}$ & 2.275 & 0.141 & 0.729 \\
\hline $\mathrm{P}$ & -2.399 & 0.766 & -0.145 \\
\hline O & 2.697 & -0.046 & 2.148 \\
\hline $\mathrm{C}$ & 1.805 & 1.840 & 0.351 \\
\hline $\mathrm{C}$ & 3.592 & -0.208 & -0.453 \\
\hline O & -1.496 & 1.819 & -0.689 \\
\hline $\mathrm{C}$ & -3.135 & 1.184 & 1.448 \\
\hline $\mathrm{C}$ & -3.841 & 0.435 & -1.178 \\
\hline $\mathrm{H}$ & -0.941 & -2.523 & -1.146 \\
\hline $\mathrm{H}$ & -1.544 & -1.134 & -2.110 \\
\hline $\mathrm{H}$ & 1.111 & -1.436 & -1.909 \\
\hline $\mathrm{H}$ & 0.447 & 0.200 & -1.557 \\
\hline $\mathrm{H}$ & 1.254 & -2.024 & 0.415 \\
\hline $\mathrm{H}$ & -0.456 & -1.540 & 1.875 \\
\hline $\mathrm{H}$ & -0.363 & 0.222 & 1.615 \\
\hline $\mathrm{H}$ & -2.343 & -1.575 & 0.506 \\
\hline $\mathrm{H}$ & 0.925 & 2.140 & 0.955 \\
\hline $\mathrm{H}$ & 1.561 & 1.955 & -0.724 \\
\hline $\mathrm{H}$ & 2.644 & 2.523 & 0.593 \\
\hline $\mathrm{H}$ & 3.904 & -1.270 & -0.373 \\
\hline $\mathrm{H}$ & 4.470 & 0.437 & -0.244 \\
\hline $\mathrm{H}$ & 3.249 & -0.014 & -1.489 \\
\hline $\mathrm{H}$ & -2.345 & 1.385 & 2.199 \\
\hline $\mathrm{H}$ & -3.769 & 0.350 & 1.812 \\
\hline $\mathrm{H}$ & -3.764 & 2.093 & 1.348 \\
\hline $\mathrm{H}$ & -3.526 & 0.141 & -2.199 \\
\hline $\mathrm{H}$ & -4.470 & 1.346 & -1.253 \\
\hline $\mathrm{H}$ & -4.449 & -0.383 & -0.741 \\
\hline \multicolumn{4}{|c|}{47} \\
\hline \multicolumn{4}{|c|}{ Structure 24, Gd complex } \\
\hline $\mathrm{P}$ & 1.989 & -1.380 & 0.868 \\
\hline 0 & 1.261 & -2.026 & -0.259 \\
\hline $\mathrm{C}$ & 2.245 & -2.454 & 2.295 \\
\hline $\mathrm{C}$ & 3.692 & -0.952 & 0.442 \\
\hline $\mathrm{P}$ & -2.036 & -1.429 & 0.582 \\
\hline 0 & -1.448 & -1.781 & -0.739 \\
\hline $\mathrm{C}$ & -3.843 & -1.331 & 0.559 \\
\hline $\mathrm{C}$ & -1.838 & -2.732 & 1.815 \\
\hline $\mathrm{H}$ & 2.804 & -3.363 & 1.991 \\
\hline $\mathrm{H}$ & 1.275 & -2.769 & 2.728 \\
\hline $\mathrm{H}$ & 2.826 & -1.925 & 3.078 \\
\hline $\mathrm{H}$ & 4.271 & -1.873 & 0.229 \\
\hline $\mathrm{H}$ & 4.179 & -0.411 & 1.280 \\
\hline $\mathrm{H}$ & 3.715 & -0.311 & -0.462 \\
\hline $\mathrm{H}$ & -4.271 & -2.321 & 0.300 \\
\hline $\mathrm{H}$ & -4.195 & -0.602 & -0.196 \\
\hline $\mathrm{H}$ & -4.229 & -1.030 & 1.554 \\
\hline $\mathrm{H}$ & -2.392 & -3.639 & 1.499 \\
\hline $\mathrm{H}$ & -2.228 & -2.402 & 2.799 \\
\hline $\mathrm{H}$ & -0.771 & -3.005 & 1.919 \\
\hline $\mathrm{Gd}$ & 0.146 & -2.448 & -2.205 \\
\hline $\mathrm{C}$ & -1.274 & 1.337 & 0.296 \\
\hline $\mathrm{C}$ & -2.468 & 1.493 & -0.669 \\
\hline $\mathrm{C}$ & -2.306 & 2.694 & -1.608 \\
\hline C & -1.008 & 2.568 & -2.412 \\
\hline $\mathrm{C}$ & 0.192 & 2.451 & -1.467 \\
\hline $\mathrm{C}$ & 0.057 & 1.267 & -0.486 \\
\hline $\mathrm{C}$ & 1.238 & 1.304 & 0.498 \\
\hline $\mathrm{C}$ & 1.242 & 0.156 & 1.524 \\
\hline $\mathrm{C}$ & -0.158 & 0.024 & 2.176 \\
\hline $\mathrm{C}$ & -1.434 & 0.153 & 1.296 \\
\hline $\mathrm{H}$ & -2.240 & 0.462 & 1.997 \\
\hline $\mathrm{H}$ & -0.202 & -0.901 & 2.791 \\
\hline $\mathrm{H}$ & -0.222 & 0.846 & 2.926 \\
\hline $\mathrm{H}$ & 1.928 & 0.476 & 2.339 \\
\hline $\mathrm{H}$ & 1.201 & 2.269 & 1.055 \\
\hline
\end{tabular}




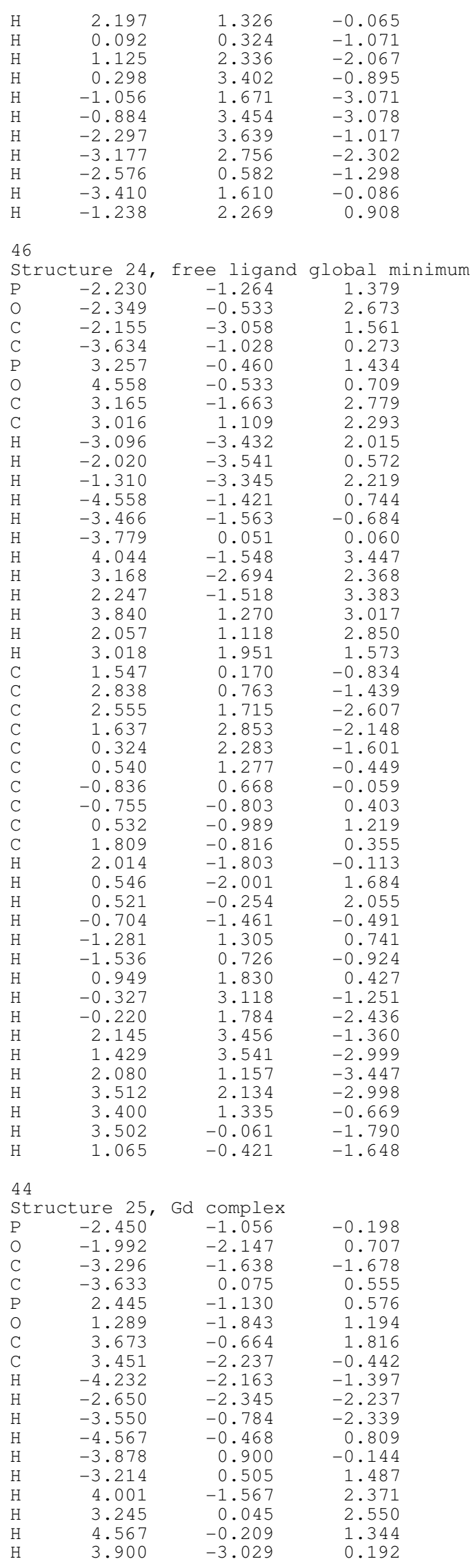




$\begin{array}{lrrr}\mathrm{H} & 4.269 & -1.681 & -0.943 \\ \mathrm{H} & 2.825 & -2.731 & -1.212 \\ \mathrm{Gd} & -0.373 & -3.282 & 1.824 \\ \mathrm{C} & 1.964 & 0.283 & -0.539 \\ \mathrm{C} & 1.321 & -0.256 & -1.835 \\ \mathrm{C} & -0.046 & -0.921 & -1.567 \\ \mathrm{C} & -1.048 & -0.033 & -0.776 \\ \mathrm{C} & -0.381 & 0.669 & 0.439 \\ \mathrm{C} & 0.948 & 1.300 & 0.024 \\ \mathrm{C} & 1.747 & 2.101 & 1.058 \\ \mathrm{C} & 3.145 & 2.253 & 0.394 \\ \mathrm{C} & 3.188 & 1.208 & -0.766 \\ \mathrm{H} & 2.003 & -0.965 & -2.354 \\ \mathrm{H} & 1.167 & 0.584 & -2.550 \\ \mathrm{H} & 0.121 & -1.881 & -1.032 \\ \mathrm{H} & -1.427 & 0.754 & -1.463 \\ \mathrm{H} & -1.054 & 1.469 & 0.826 \\ \mathrm{H} & -0.225 & -0.035 & 1.283 \\ \mathrm{H} & 0.708 & 2.021 & -0.794 \\ \mathrm{H} & 1.811 & 1.549 & 2.022 \\ \mathrm{H} & 1.279 & 3.090 & 1.266 \\ \mathrm{H} & 3.275 & 3.282 & -0.015 \\ \mathrm{H} & 3.959 & 2.085 & 1.134 \\ \mathrm{H} & 4.158 & 0.663 & -0.807 \\ \mathrm{H} & 3.082 & 1.742 & -1.738 \\ \mathrm{H} & -0.499 & -1.201 & -2.546\end{array}$

43

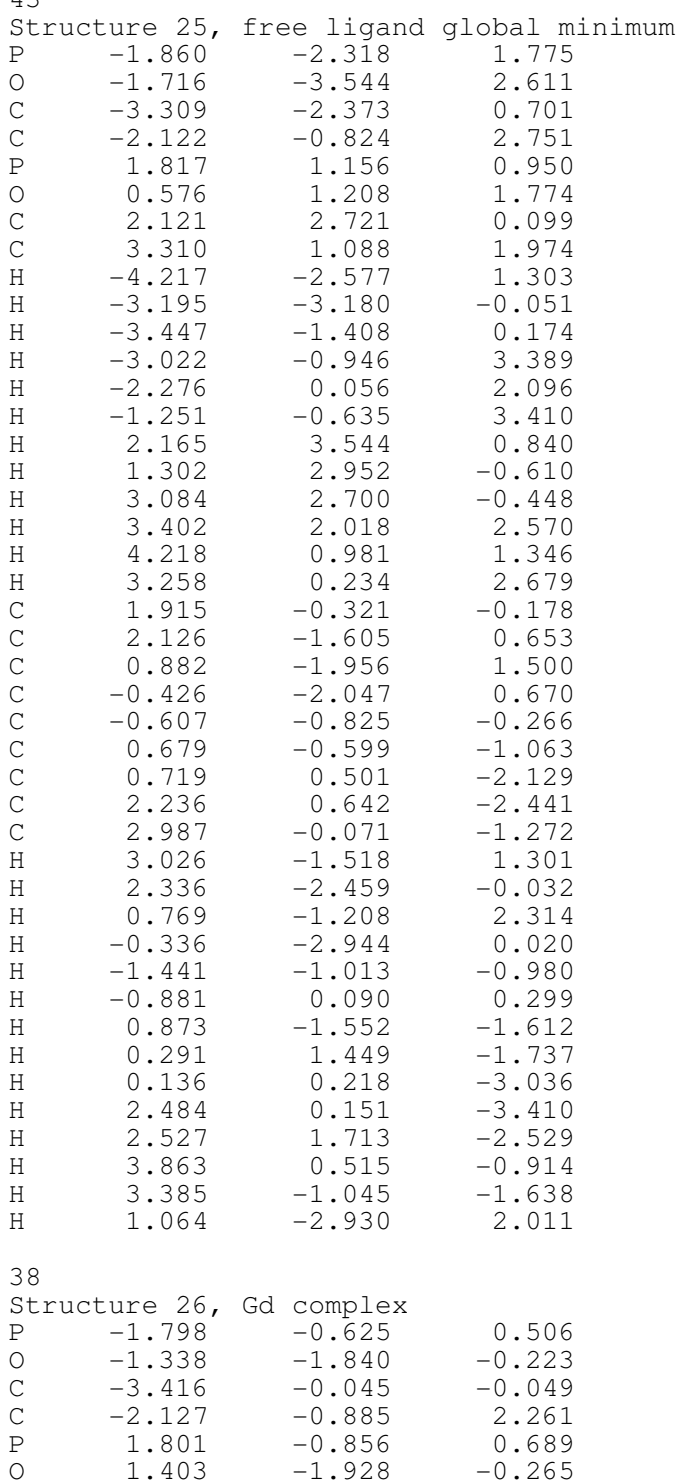




\begin{tabular}{|c|c|c|c|}
\hline $\mathrm{C}$ & 1.701 & -1.365 & 2.415 \\
\hline $\mathrm{C}$ & 3.546 & -0.404 & 0.563 \\
\hline $\mathrm{H}$ & -4.183 & -0.823 & 0.144 \\
\hline $\mathrm{H}$ & -3.399 & 0.163 & -1.138 \\
\hline $\mathrm{H}$ & -3.704 & 0.881 & 0.490 \\
\hline $\mathrm{H}$ & -2.838 & -1.726 & 2.393 \\
\hline $\mathrm{H}$ & -2.568 & 0.027 & 2.711 \\
\hline $\mathrm{H}$ & -1.194 & -1.125 & 2.804 \\
\hline $\mathrm{H}$ & 2.484 & -2.120 & 2.633 \\
\hline $\mathrm{H}$ & 0.717 & -1.825 & 2.623 \\
\hline $\mathrm{H}$ & 1.846 & -0.495 & 3.088 \\
\hline $\mathrm{H}$ & 4.183 & -1.279 & 0.806 \\
\hline $\mathrm{H}$ & 3.785 & 0.418 & 1.267 \\
\hline $\mathrm{H}$ & 3.785 & -0.077 & -0.469 \\
\hline $\mathrm{Gd}$ & -0.022 & -3.266 & -1.418 \\
\hline $\mathrm{C}$ & -0.887 & 1.640 & -0.908 \\
\hline $\mathrm{C}$ & 0.291 & 2.621 & -0.820 \\
\hline $\mathrm{C}$ & 1.364 & 1.525 & -0.749 \\
\hline $\mathrm{C}$ & 0.895 & 0.722 & 0.506 \\
\hline $\mathrm{C}$ & -0.686 & 0.821 & 0.403 \\
\hline $\mathrm{C}$ & -0.484 & 0.815 & -2.157 \\
\hline $\mathrm{C}$ & 1.065 & 0.760 & -2.062 \\
\hline $\mathrm{H}$ & -1.880 & 2.140 & -0.994 \\
\hline $\mathrm{H}$ & 0.260 & 3.265 & 0.089 \\
\hline $\mathrm{H}$ & 0.387 & 3.266 & -1.725 \\
\hline $\mathrm{H}$ & 2.404 & 1.922 & -0.681 \\
\hline $\mathrm{H}$ & -1.005 & 1.493 & 1.230 \\
\hline $\mathrm{H}$ & 1.185 & 1.333 & 1.389 \\
\hline $\mathrm{H}$ & -0.798 & 1.341 & -3.088 \\
\hline $\mathrm{H}$ & -0.940 & -0.199 & -2.176 \\
\hline $\mathrm{H}$ & 1.455 & -0.280 & -2.069 \\
\hline $\mathrm{H}$ & 1.524 & 1.290 & -2.929 \\
\hline \multicolumn{4}{|c|}{37} \\
\hline \multicolumn{4}{|c|}{ Structure 26, } \\
\hline $\mathrm{P}$ & -1.890 & -1.472 & -0.284 \\
\hline 0 & -3.087 & -0.606 & -0.485 \\
\hline $\mathrm{C}$ & -1.900 & -2.303 & 1.314 \\
\hline $\mathrm{C}$ & -1.928 & -2.916 & -1.378 \\
\hline $\mathrm{P}$ & -0.680 & 1.483 & 1.264 \\
\hline 0 & -0.795 & 0.574 & 2.439 \\
\hline $\mathrm{C}$ & -2.237 & 2.244 & 0.764 \\
\hline $\mathrm{C}$ & 0.313 & 2.956 & 1.603 \\
\hline $\mathrm{H}$ & -2.909 & -2.720 & 1.506 \\
\hline $\mathrm{H}$ & -1.660 & -1.602 & 2.135 \\
\hline $\mathrm{H}$ & -1.180 & -3.145 & 1.332 \\
\hline $\mathrm{H}$ & -1.067 & -3.586 & -1.178 \\
\hline $\mathrm{H}$ & -1.892 & -2.594 & -2.439 \\
\hline $\mathrm{H}$ & -2.864 & -3.489 & -1.217 \\
\hline $\mathrm{H}$ & -2.584 & 2.946 & 1.550 \\
\hline $\mathrm{H}$ & -3.023 & 1.480 & 0.621 \\
\hline $\mathrm{H}$ & -2.107 & 2.808 & -0.182 \\
\hline $\mathrm{H}$ & -0.159 & 3.555 & 2.408 \\
\hline $\mathrm{H}$ & 0.396 & 3.586 & 0.693 \\
\hline $\mathrm{H}$ & 1.331 & 2.668 & 1.934 \\
\hline $\mathrm{C}$ & 0.948 & -1.470 & -0.796 \\
\hline $\mathrm{C}$ & 1.961 & -0.395 & -1.226 \\
\hline $\mathrm{C}$ & 1.654 & 0.548 & -0.051 \\
\hline $\mathrm{C}$ & 0.112 & 0.755 & -0.211 \\
\hline $\mathrm{C}$ & -0.361 & -0.630 & -0.812 \\
\hline $\mathrm{C}$ & 1.441 & -1.762 & 0.644 \\
\hline $\mathrm{C}$ & 1.984 & -0.391 & 1.132 \\
\hline $\mathrm{H}$ & 0.934 & -2.363 & -1.463 \\
\hline $\mathrm{H}$ & 1.733 & 0.056 & -2.220 \\
\hline $\mathrm{H}$ & 3.012 & -0.765 & -1.225 \\
\hline $\mathrm{H}$ & 2.249 & 1.491 & -0.061 \\
\hline $\mathrm{H}$ & -0.547 & -0.419 & -1.887 \\
\hline $\mathrm{H}$ & -0.017 & 1.507 & -1.021 \\
\hline $\mathrm{H}$ & 2.256 & -2.522 & 0.632 \\
\hline $\mathrm{H}$ & 0.639 & -2.140 & 1.309 \\
\hline $\mathrm{H}$ & 1.536 & -0.070 & 2.096 \\
\hline $\mathrm{H}$ & 3.087 & -0.444 & 1.283 \\
\hline \multicolumn{4}{|c|}{41} \\
\hline \multicolumn{4}{|c|}{ St } \\
\hline $\mathrm{P}$ & 1.605 & -1.401 & -0.702 \\
\hline 0 & 1.206 & -2.569 & 0.129 \\
\hline $\mathrm{C}$ & 3.346 & -0.951 & -0.542 \\
\hline $\mathrm{C}$ & 1.508 & -1.732 & -2.475 \\
\hline $\mathrm{P}$ & -2.048 & -1.153 & -0.658 \\
\hline 0 & -1.493 & -2.469 & -0.233 \\
\hline
\end{tabular}




\begin{tabular}{|c|c|c|c|}
\hline $\mathrm{C}$ & 305 & 1271 & $2 \quad 125$ \\
\hline $\mathrm{C}$ & -3.185 & -0.462 & $\begin{array}{r}-2.123 \\
0.563\end{array}$ \\
\hline $\mathrm{H}$ & 3.984 & -1.765 & -0.942 \\
\hline $\mathrm{H}$ & 3.619 & -0.794 & 0.519 \\
\hline $\mathrm{H}$ & 3.560 & -0.022 & -1.110 \\
\hline $\mathrm{H}$ & 2.221 & -2.536 & -2.749 \\
\hline $\mathrm{H}$ & 1.759 & -0.820 & -3.055 \\
\hline $\mathrm{H}$ & 0.487 & -2.067 & -2.749 \\
\hline $\mathrm{H}$ & -3.936 & -1.971 & -1.934 \\
\hline $\mathrm{H}$ & -2.504 & -1.658 & -2.982 \\
\hline $\mathrm{H}$ & -3.510 & -0.281 & -2.393 \\
\hline $\mathrm{H}$ & -3.984 & -1.199 & 0.785 \\
\hline $\mathrm{H}$ & -3.663 & 0.464 & 0.184 \\
\hline $\mathrm{H}$ & -2.655 & -0.233 & 1.508 \\
\hline $\mathrm{Gd}$ & -0.317 & -4.028 & 0.953 \\
\hline $\mathrm{C}$ & -1.423 & 1. 444 & -0.952 \\
\hline $\mathrm{C}$ & -1.170 & 2.237 & 0.098 \\
\hline $\mathrm{C}$ & -0.161 & 1.938 & 1.182 \\
\hline $\mathrm{C}$ & 0.351 & 0.470 & 1.092 \\
\hline $\mathrm{C}$ & 0.595 & 0.103 & -0.396 \\
\hline $\mathrm{C}$ & -0.778 & 0.091 & -1.141 \\
\hline $\mathrm{C}$ & 0.959 & 2.994 & 1.087 \\
\hline $\mathrm{C}$ & 1.552 & 0.267 & 2.033 \\
\hline $\mathrm{H}$ & -2.154 & 1.773 & -1.711 \\
\hline $\mathrm{H}$ & -1.699 & 3.203 & 0.174 \\
\hline $\mathrm{H}$ & -0.675 & 2.065 & 2.164 \\
\hline $\mathrm{H}$ & -0.453 & -0.197 & 1.477 \\
\hline $\mathrm{H}$ & 1.183 & 0.934 & -0.839 \\
\hline $\mathrm{H}$ & -0.572 & -0.017 & -2.227 \\
\hline $\mathrm{H}$ & 0.547 & 4.027 & 1.126 \\
\hline $\mathrm{H}$ & 1.539 & 2.912 & 0.141 \\
\hline $\mathrm{H}$ & 1.681 & 2.917 & 1.929 \\
\hline $\mathrm{H}$ & 1.334 & 0.650 & 3.055 \\
\hline $\mathrm{H}$ & 2.464 & 0.790 & 1.672 \\
\hline $\mathrm{H}$ & 1.808 & -0.809 & 2.156 \\
\hline 40 & & & \\
\hline St & cure 27 , & free ligand & global minimum \\
\hline $\mathrm{P}$ & -1.391 & 1.815 & 0.137 \\
\hline 0 & -0.430 & 2.956 & 0.089 \\
\hline $\mathrm{C}$ & -2.102 & 1.586 & 1.780 \\
\hline $\mathrm{C}$ & -2.847 & 2.158 & -0.878 \\
\hline $\mathrm{P}$ & 1.295 & 0.105 & 1.678 \\
\hline 0 & 0.263 & -0.572 & 2.514 \\
\hline $\mathrm{C}$ & 1.690 & 1.783 & 2.222 \\
\hline $\mathrm{C}$ & 2.930 & -0.649 & 1.832 \\
\hline $\mathrm{H}$ & -2.715 & 2.471 & 2.048 \\
\hline $\mathrm{H}$ & -1.309 & 1.481 & 2.544 \\
\hline $\mathrm{H}$ & -2.749 & 0.690 & 1.824 \\
\hline $\mathrm{H}$ & -3.221 & 3.179 & -0.658 \\
\hline $\mathrm{H}$ & -3.667 & 1.445 & -0.671 \\
\hline $\mathrm{H}$ & -2.585 & 2.106 & -1.954 \\
\hline $\mathrm{H}$ & 2.135 & 1.756 & 3.237 \\
\hline $\mathrm{H}$ & 0.782 & 2.414 & 2.266 \\
\hline $\mathrm{H}$ & 2.418 & 2.254 & 1.530 \\
\hline $\mathrm{H}$ & 3.273 & -0.595 & 2.885 \\
\hline $\mathrm{H}$ & 3.667 & -0.120 & 1.194 \\
\hline $\mathrm{H}$ & 2.894 & -1.718 & 1.536 \\
\hline $\mathrm{C}$ & 1.506 & -1.020 & -0.764 \\
\hline $\mathrm{C}$ & 0.813 & -2.082 & -1.188 \\
\hline $\mathrm{C}$ & -0.689 & -2.185 & -1.166 \\
\hline $\mathrm{C}$ & -1.325 & -1.083 & -0.269 \\
\hline $\mathrm{C}$ & -0.620 & 0.282 & -0.521 \\
\hline $\mathrm{C}$ & 0.892 & 0.198 & -0.115 \\
\hline $\mathrm{C}$ & -1.175 & -2.164 & -2.631 \\
\hline $\mathrm{C}$ & -2.851 & -1.099 & -0.484 \\
\hline $\mathrm{H}$ & 2.602 & -1.023 & -0.892 \\
\hline $\mathrm{H}$ & 1.359 & -2.928 & -1.641 \\
\hline $\mathrm{H}$ & -0.950 & -3.179 & -0.730 \\
\hline $\mathrm{H}$ & -1.150 & -1.373 & 0.791 \\
\hline $\mathrm{H}$ & -0.626 & 0.422 & -1.622 \\
\hline $\mathrm{H}$ & 1.415 & 1.078 & -0.547 \\
\hline $\mathrm{H}$ & -0.670 & -2.949 & -3.237 \\
\hline $\mathrm{H}$ & -0.973 & -1.190 & -3.129 \\
\hline $\mathrm{H}$ & -2.266 & -2.363 & -2.717 \\
\hline $\mathrm{H}$ & -3.253 & -2.136 & -0.445 \\
\hline $\mathrm{H}$ & -3.142 & -0.670 & -1.468 \\
\hline $\mathrm{H}$ & -3.398 & -0.537 & 0.301 \\
\hline
\end{tabular}




\begin{tabular}{|c|c|c|c|}
\hline $\mathrm{P}$ & -2.550 & -0.454 & 0.443 \\
\hline 0 & -1.688 & -1.659 & 0.292 \\
\hline $\mathrm{C}$ & -4.302 & -0.799 & 0.181 \\
\hline $\mathrm{C}$ & -2.523 & 0.230 & 2.109 \\
\hline $\mathrm{P}$ & 2.596 & -0.444 & 0.917 \\
\hline 0 & 1.677 & -1.597 & 0.703 \\
\hline $\mathrm{C}$ & 2.901 & -0.080 & 2.656 \\
\hline $\mathrm{C}$ & 4.254 & -0.738 & 0.267 \\
\hline $\mathrm{H}$ & -4.650 & -1.566 & 0.902 \\
\hline $\mathrm{H}$ & -4.465 & -1.180 & -0.848 \\
\hline $\mathrm{H}$ & -4.904 & 0.123 & 0.321 \\
\hline $\mathrm{H}$ & -2.972 & -0.493 & 2.819 \\
\hline $\mathrm{H}$ & -3.101 & 1.176 & 2.151 \\
\hline $\mathrm{H}$ & -1.480 & 0.425 & 2.429 \\
\hline $\mathrm{H}$ & 3.384 & -0.950 & 3.145 \\
\hline $\mathrm{H}$ & 1.945 & 0.129 & 3.177 \\
\hline $\mathrm{H}$ & 3.567 & 0.801 & 2.756 \\
\hline $\mathrm{H}$ & 4.705 & -1.621 & 0.765 \\
\hline $\mathrm{H}$ & 4.904 & 0.143 & 0.448 \\
\hline $\mathrm{H}$ & 4.212 & -0.933 & -0.823 \\
\hline $\mathrm{Gd}$ & 0.003 & -3.133 & 0.627 \\
\hline $\mathrm{C}$ & -0.279 & 1.946 & 0.500 \\
\hline $\mathrm{C}$ & 0.982 & 1.793 & 0.926 \\
\hline $\mathrm{C}$ & 2.084 & 1.128 & 0.137 \\
\hline $\mathrm{C}$ & 1.675 & 0.963 & -1.352 \\
\hline $\mathrm{C}$ & 0.218 & 0.468 & -1.459 \\
\hline $\mathrm{C}$ & -0.788 & 1.451 & -0.830 \\
\hline $\mathrm{C}$ & 1.875 & 2.282 & -2.124 \\
\hline $\mathrm{C}$ & -2.218 & 0.872 & -0.753 \\
\hline $\mathrm{H}$ & -0.991 & 2.507 & 1.129 \\
\hline $\mathrm{H}$ & 1.260 & 2.222 & 1.904 \\
\hline $\mathrm{H}$ & 2.957 & 1.814 & 0.171 \\
\hline $\mathrm{H}$ & 2.338 & 0.203 & -1.830 \\
\hline $\mathrm{H}$ & 0.136 & -0.523 & -0.969 \\
\hline $\mathrm{H}$ & -0.044 & 0.300 & -2.529 \\
\hline $\mathrm{H}$ & -0.843 & 2.345 & -1.494 \\
\hline $\mathrm{H}$ & 1.525 & 2.195 & -3.177 \\
\hline $\mathrm{H}$ & 2.950 & 2.569 & -2.163 \\
\hline $\mathrm{H}$ & 1.329 & 3.133 & -1.660 \\
\hline $\mathrm{H}$ & -2.505 & 0.492 & -1.756 \\
\hline $\mathrm{H}$ & -2.927 & 1.696 & -0.526 \\
\hline \multicolumn{4}{|c|}{40} \\
\hline \multicolumn{4}{|c|}{ Str } \\
\hline $\mathrm{P}$ & 2.986 & -1.977 & 0.283 \\
\hline 0 & 2.474 & -3.085 & -0.574 \\
\hline $\mathrm{C}$ & 3.728 & -2.549 & 1.825 \\
\hline $\mathrm{C}$ & 4.291 & -1.000 & -0.484 \\
\hline $\mathrm{P}$ & -3.131 & 0.216 & -0.067 \\
\hline 0 & -3.744 & 1.380 & 0.635 \\
\hline $\mathrm{C}$ & -2.590 & -1.092 & 1.051 \\
\hline $\mathrm{C}$ & -4.302 & -0.661 & -1.128 \\
\hline $\mathrm{H}$ & 4.576 & -3.232 & 1.608 \\
\hline $\mathrm{H}$ & 2.976 & -3.097 & 2.429 \\
\hline $\mathrm{H}$ & 4.105 & -1.690 & 2.416 \\
\hline $\mathrm{H}$ & 5.173 & -1.641 & -0.690 \\
\hline $\mathrm{H}$ & 4.600 & -0.172 & 0.187 \\
\hline $\mathrm{H}$ & 3.936 & -0.571 & -1.443 \\
\hline $\mathrm{H}$ & -3.465 & -1.505 & 1.595 \\
\hline $\mathrm{H}$ & -1.876 & -0.700 & 1.801 \\
\hline $\mathrm{H}$ & -2.106 & -1.917 & 0.491 \\
\hline $\mathrm{H}$ & -5.173 & -1.001 & -0.531 \\
\hline $\mathrm{H}$ & -3.825 & -1.547 & -1.593 \\
\hline $\mathrm{H}$ & -4.666 & 0.012 & -1.931 \\
\hline $\mathrm{C}$ & 0.223 & -0.829 & -1.223 \\
\hline $\mathrm{C}$ & -1.017 & -0.534 & -1.639 \\
\hline $\mathrm{C}$ & -1.785 & 0.691 & -1.210 \\
\hline $\mathrm{C}$ & -0.822 & 1.761 & -0.637 \\
\hline $\mathrm{C}$ & 0.166 & 1.122 & 0.354 \\
\hline $\mathrm{C}$ & 1.044 & 0.024 & -0.286 \\
\hline $\mathrm{C}$ & -0.072 & 2.489 & -1.770 \\
\hline $\mathrm{C}$ & 1.722 & -0.795 & 0.834 \\
\hline $\mathrm{H}$ & 0.701 & -1.737 & -1.624 \\
\hline $\mathrm{H}$ & -1.511 & -1.213 & -2.355 \\
\hline $\mathrm{H}$ & -2.256 & 1.126 & -2.117 \\
\hline $\mathrm{H}$ & -1.418 & 2.533 & -0.094 \\
\hline $\mathrm{H}$ & -0.401 & 0.698 & 1. 211 \\
\hline $\mathrm{H}$ & 0.821 & 1.911 & 0.791 \\
\hline $\mathrm{H}$ & 1.834 & 0.521 & -0.894 \\
\hline $\mathrm{H}$ & 0.653 & 3.232 & -1.368 \\
\hline $\mathrm{H}$ & -0.775 & 3.047 & -2.429 \\
\hline
\end{tabular}




\begin{tabular}{|c|c|c|c|}
\hline $\begin{array}{l}\mathrm{H} \\
\mathrm{H} \\
\mathrm{H}\end{array}$ & $\begin{array}{l}0.497 \\
0.956 \\
2.210\end{array}$ & $\begin{array}{r}1.789 \\
-1.358 \\
-0.102\end{array}$ & $\begin{array}{r}-2.422 \\
1.408 \\
1.550\end{array}$ \\
\hline \multicolumn{4}{|c|}{41} \\
\hline \multicolumn{4}{|c|}{ Gd complex } \\
\hline $\mathrm{P}$ & 1.554 & -0.853 & -0.889 \\
\hline 0 & 1.447 & -2.191 & -0.245 \\
\hline $\mathrm{C}$ & 3.163 & -0.062 & -0.674 \\
\hline $\mathrm{C}$ & 1.459 & -0.931 & -2.691 \\
\hline $\mathrm{P}$ & -2.073 & -1.398 & -0.770 \\
\hline $\mathrm{O}$ & -1.242 & -2.615 & -0.552 \\
\hline $\mathrm{C}$ & -3.133 & -1.522 & -2.227 \\
\hline $\mathrm{C}$ & -3.274 & -1.140 & 0.553 \\
\hline $\mathrm{H}$ & 3.948 & -0.661 & -1.179 \\
\hline $\mathrm{H}$ & 3.428 & 0.015 & 0.398 \\
\hline $\mathrm{H}$ & 3.155 & 0.956 & -1.115 \\
\hline $\mathrm{H}$ & 2.317 & -1.508 & -3.091 \\
\hline $\mathrm{H}$ & 1.483 & 0.089 & -3.126 \\
\hline $\mathrm{H}$ & 0.525 & -1.438 & -3.006 \\
\hline $\mathrm{H}$ & -3.805 & -2.400 & -2.133 \\
\hline $\mathrm{H}$ & -2.513 & -1.647 & -3.138 \\
\hline $\mathrm{H}$ & -3.754 & -0.610 & -2.339 \\
\hline $\mathrm{H}$ & -3.895 & -2.051 & 0.675 \\
\hline $\mathrm{H}$ & -3.948 & -0.291 & 0.320 \\
\hline $\mathrm{H}$ & -2.763 & -0.939 & 1.515 \\
\hline $\mathrm{Gd}$ & 0.292 & -4.041 & 0.361 \\
\hline $\mathrm{C}$ & -1.906 & 1.953 & 0.474 \\
\hline $\mathrm{C}$ & -2.009 & 1.289 & -0.689 \\
\hline $\mathrm{C}$ & -1.106 & 0.140 & -1.075 \\
\hline $\mathrm{C}$ & 0.261 & 0.329 & -0.343 \\
\hline $\mathrm{C}$ & 0.002 & 0.384 & 1.183 \\
\hline $\mathrm{C}$ & -0.828 & 1.653 & 1.486 \\
\hline $\mathrm{C}$ & -2.825 & 3.096 & 0.825 \\
\hline $\mathrm{C}$ & 1.262 & 0.409 & 2.067 \\
\hline $\mathrm{H}$ & -2.795 & 1.576 & -1.408 \\
\hline $\mathrm{H}$ & -0.920 & 0.230 & -2.165 \\
\hline $\mathrm{H}$ & 0.640 & 1.332 & -0.638 \\
\hline $\mathrm{H}$ & -0.585 & -0.514 & 1.478 \\
\hline $\mathrm{H}$ & -1.290 & 1.553 & 2.495 \\
\hline $\mathrm{H}$ & -0.151 & 2.535 & 1.533 \\
\hline $\mathrm{H}$ & -3.592 & 3.277 & 0.041 \\
\hline $\mathrm{H}$ & -2.253 & 4.041 & 0.955 \\
\hline $\mathrm{H}$ & -3.366 & 2.894 & 1.776 \\
\hline $\mathrm{H}$ & 1.949 & 1.237 & 1.786 \\
\hline $\mathrm{H}$ & 1.830 & -0.546 & 2.013 \\
\hline $\mathrm{H}$ & 0.998 & 0.553 & 3.138 \\
\hline \multicolumn{4}{|c|}{40} \\
\hline St & ture 29, & free ligand & global minimu \\
\hline $\mathrm{P}$ & -1.754 & -1.443 & -1.031 \\
\hline 0 & -2.701 & -1.562 & 0.116 \\
\hline $\mathrm{C}$ & -0.976 & -3.017 & -1.449 \\
\hline $\mathrm{C}$ & -2.632 & -1.007 & -2.549 \\
\hline $\mathrm{P}$ & 0.684 & -1.499 & 1.559 \\
\hline 0 & 1.580 & -2.267 & 0.647 \\
\hline $\mathrm{C}$ & -0.605 & -2.505 & 2.329 \\
\hline $\mathrm{C}$ & 1.530 & -0.908 & 3.043 \\
\hline $\mathrm{H}$ & -1.755 & -3.755 & -1.729 \\
\hline $\mathrm{H}$ & -0.416 & -3.423 & -0.586 \\
\hline $\mathrm{H}$ & -0.277 & -2.912 & -2.300 \\
\hline $\mathrm{H}$ & -3.495 & -1.690 & -2.689 \\
\hline $\mathrm{H}$ & -1.981 & -1.094 & -3.439 \\
\hline $\mathrm{H}$ & -3.016 & 0.031 & -2.484 \\
\hline $\mathrm{H}$ & -0.144 & -3.265 & 2.993 \\
\hline $\mathrm{H}$ & -1.203 & -3.039 & 1.567 \\
\hline $\mathrm{H}$ & -1.281 & -1.870 & 2.937 \\
\hline $\mathrm{H}$ & 1.922 & -1.768 & 3.623 \\
\hline $\mathrm{H}$ & 0.833 & -0.335 & 3.688 \\
\hline $\mathrm{H}$ & 2.387 & -0.259 & 2.769 \\
\hline $\mathrm{C}$ & 1.553 & 1.686 & 0.060 \\
\hline $\mathrm{C}$ & 0.776 & 1.139 & 1.005 \\
\hline $\mathrm{C}$ & -0.138 & -0.044 & 0.789 \\
\hline $\mathrm{C}$ & -0.510 & -0.126 & -0.731 \\
\hline $\mathrm{C}$ & 0.773 & -0.115 & -1.605 \\
\hline $\mathrm{C}$ & 1.534 & 1.211 & -1.368 \\
\hline $\mathrm{C}$ & 2.433 & 2.878 & 0.337 \\
\hline $\mathrm{C}$ & 0.542 & -0.223 & -3.125 \\
\hline $\mathrm{H}$ & 0.788 & 1.579 & 2.017 \\
\hline $\mathrm{H}$ & -1.067 & 0.174 & 1.359 \\
\hline $\mathrm{H}$ & -1.038 & 0.824 & -0.963 \\
\hline
\end{tabular}




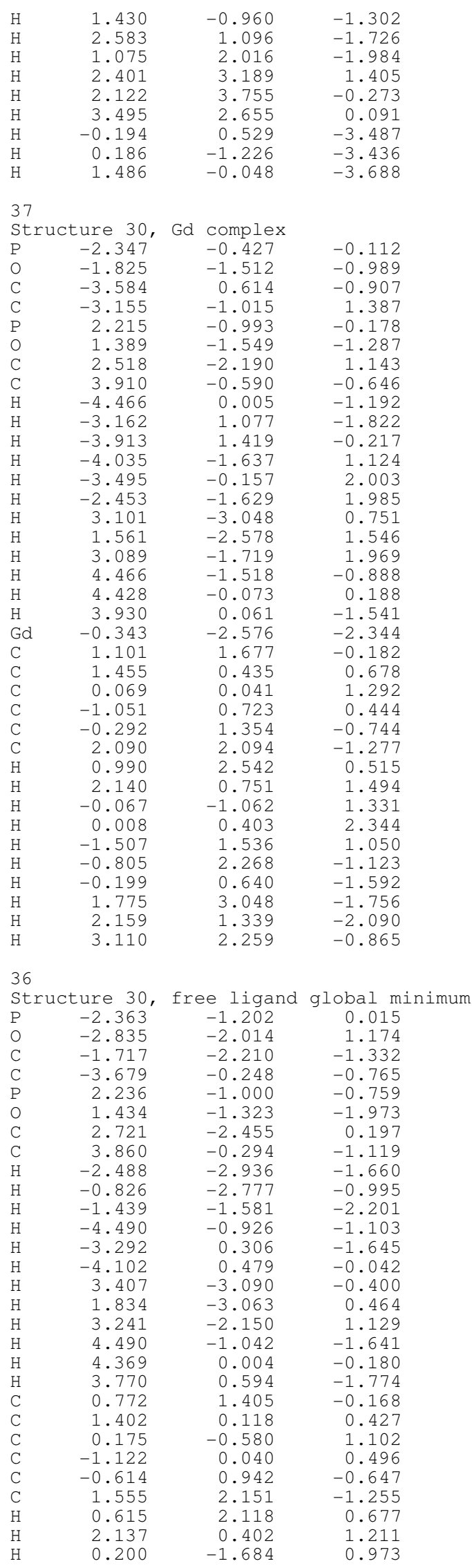




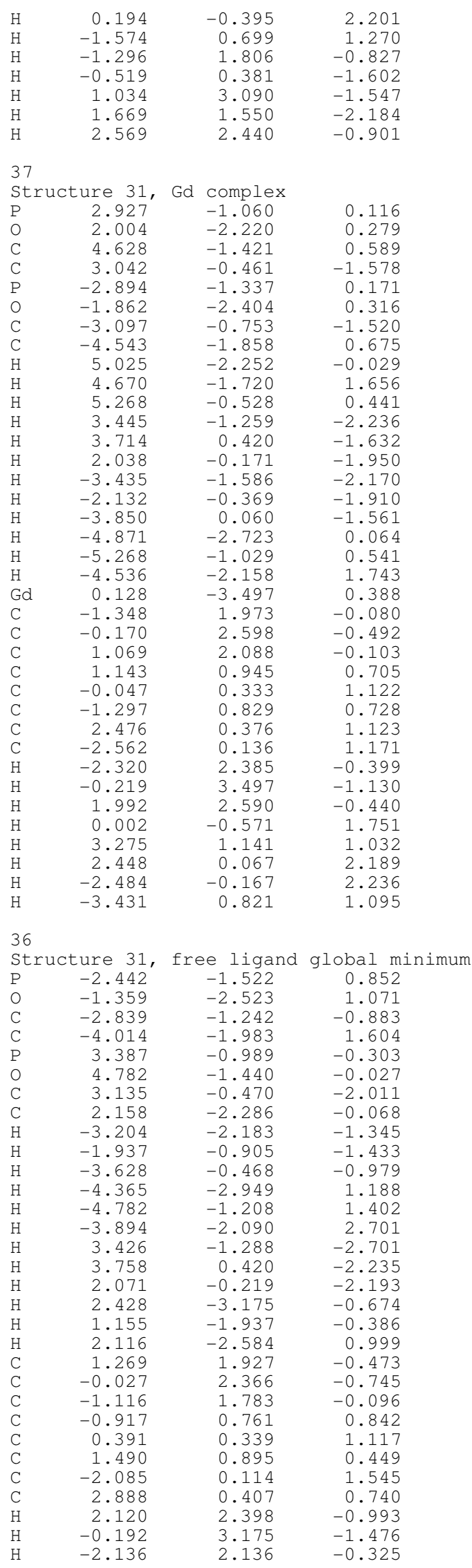




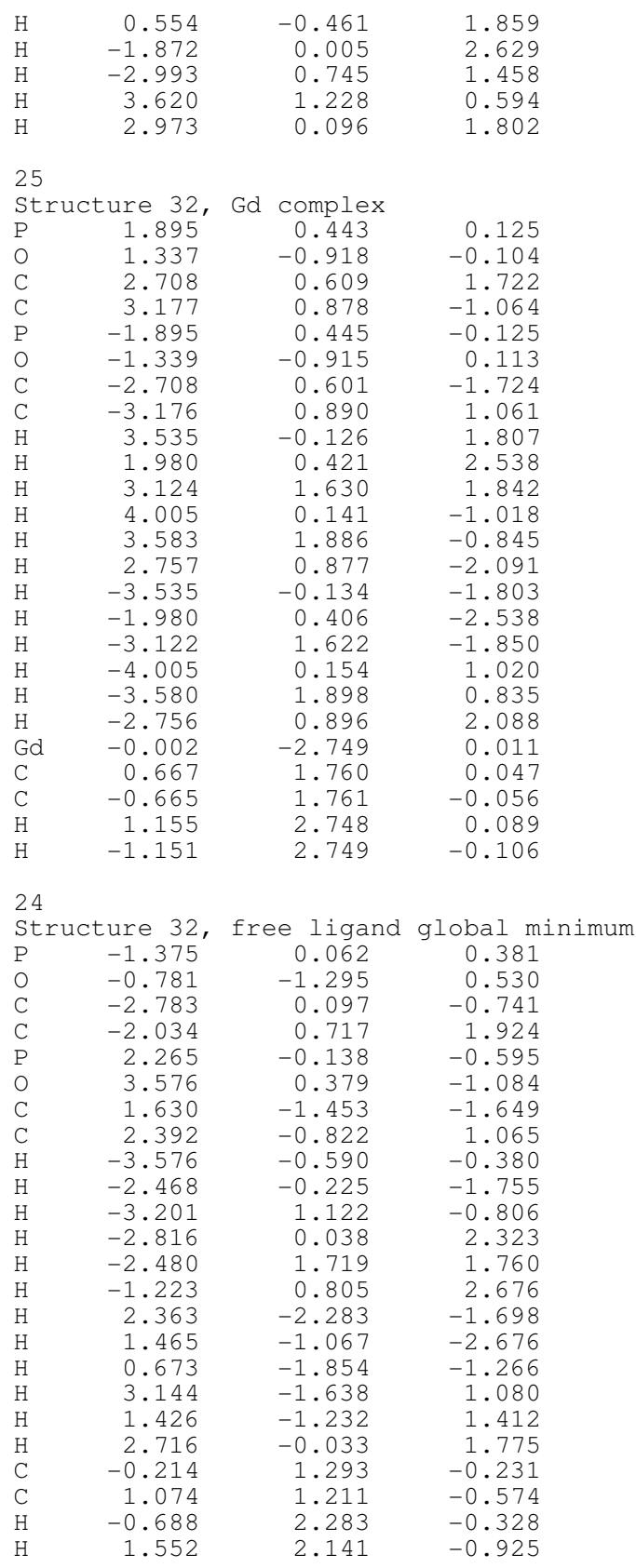




\begin{tabular}{|c|c|c|c|}
\hline \multicolumn{4}{|l|}{39} \\
\hline \multicolumn{4}{|c|}{ Gd complex } \\
\hline $\mathrm{C}$ & 3.685 & 0.313 & -0.792 \\
\hline $\mathrm{P}$ & -2.437 & -1.107 & -0.504 \\
\hline $\mathrm{P}$ & 2.562 & -0.874 & -0.038 \\
\hline 0 & -1.333 & -1.696 & -1.314 \\
\hline $\mathrm{C}$ & -3.826 & -0.507 & -1.485 \\
\hline $\mathrm{C}$ & -3.229 & -2.313 & 0.582 \\
\hline 0 & 2.029 & -1.895 & -0.983 \\
\hline $\mathrm{C}$ & 3.485 & -1.570 & 1.343 \\
\hline $\mathrm{Gd}$ & 0.384 & -2.962 & -2.126 \\
\hline $\mathrm{C}$ & 1.235 & 0.088 & 0.735 \\
\hline $\mathrm{C}$ & 0.382 & 0.825 & -0.318 \\
\hline $\mathrm{C}$ & -1.000 & 1.334 & 0.164 \\
\hline $\mathrm{C}$ & -1.655 & 2.125 & -0.990 \\
\hline $\mathrm{C}$ & -1.939 & 0.202 & 0.655 \\
\hline $\mathrm{C}$ & -0.791 & 2.319 & 1.338 \\
\hline $\mathrm{H}$ & 4.585 & -0.208 & -1.177 \\
\hline $\mathrm{H}$ & 3.186 & 0.828 & -1.638 \\
\hline $\mathrm{H}$ & 4.002 & 1.072 & -0.048 \\
\hline $\mathrm{H}$ & -4.300 & -1.354 & -2.022 \\
\hline $\mathrm{H}$ & -4.585 & -0.031 & -0.832 \\
\hline $\mathrm{H}$ & -3.485 & 0.231 & -2.237 \\
\hline $\mathrm{H}$ & -3.655 & -3.142 & -0.018 \\
\hline $\mathrm{H}$ & -2.484 & -2.734 & 1.288 \\
\hline $\mathrm{H}$ & -4.044 & -1.836 & 1.164 \\
\hline $\mathrm{H}$ & 4.365 & -2.132 & 0.967 \\
\hline $\mathrm{H}$ & 3.838 & -0.763 & 2.017 \\
\hline $\mathrm{H}$ & 2.840 & -2.263 & 1.920 \\
\hline $\mathrm{H}$ & 1.680 & 0.805 & 1.455 \\
\hline $\mathrm{H}$ & 0.616 & -0.625 & 1.315 \\
\hline $\mathrm{H}$ & 0.969 & 1.693 & -0.698 \\
\hline $\mathrm{H}$ & 0.227 & 0.169 & -1.202 \\
\hline $\mathrm{H}$ & -1.709 & 1.528 & -1.926 \\
\hline $\mathrm{H}$ & -2.689 & 2.448 & -0.737 \\
\hline $\mathrm{H}$ & -1.078 & 3.044 & -1.236 \\
\hline $\mathrm{H}$ & -1.474 & -0.302 & 1.526 \\
\hline $\mathrm{H}$ & -2.874 & 0.654 & 1.045 \\
\hline $\mathrm{H}$ & -0.093 & 3.142 & 1.069 \\
\hline $\mathrm{H}$ & -0.373 & 1.814 & 2.237 \\
\hline $\mathrm{H}$ & -1.748 & 2.793 & 1.651 \\
\hline 38 & & & \\
\hline St & ture 33, & free ligand & global minimum \\
\hline $\mathrm{C}$ & 2.646 & -0.661 & 0.415 \\
\hline $\mathrm{P}$ & -2.095 & -1.525 & 0.302 \\
\hline $\mathrm{P}$ & 2.363 & 0.928 & -0.388 \\
\hline O & -0.659 & -1.899 & 0.434 \\
\hline $\mathrm{C}$ & -2.956 & -2.463 & -0.980 \\
\hline $\mathrm{C}$ & -3.087 & -1.889 & 1.762 \\
\hline 0 & 2.962 & 2.088 & 0.331 \\
\hline $\mathrm{C}$ & 3.017 & 0.688 & -2.051 \\
\hline $\mathrm{C}$ & 0.573 & 1.063 & -0.642 \\
\hline $\mathrm{C}$ & -0.188 & 1.216 & 0.687 \\
\hline $\mathrm{C}$ & -1.729 & 1.347 & 0.570 \\
\hline $\mathrm{C}$ & -2.075 & 2.668 & -0.158 \\
\hline $\mathrm{C}$ & -2.391 & 0.190 & -0.220 \\
\hline $\mathrm{C}$ & -2.318 & 1.448 & 1.995 \\
\hline $\mathrm{H}$ & 3.734 & -0.865 & 0.476 \\
\hline $\mathrm{H}$ & 2.232 & -0.653 & 1. 444 \\
\hline $\mathrm{H}$ & 2.163 & -1.477 & -0.160 \\
\hline $\mathrm{H}$ & -2.909 & -3.548 & -0.753 \\
\hline $\mathrm{H}$ & -2.480 & -2.287 & -1.966 \\
\hline $\mathrm{H}$ & -4.021 & -2.156 & -1.036 \\
\hline $\mathrm{H}$ & -3.157 & -2.987 & 1.906 \\
\hline $\mathrm{H}$ & -4.111 & -1.480 & 1.647 \\
\hline $\mathrm{H}$ & -2.624 & -1.454 & 2.669 \\
\hline $\mathrm{H}$ & 4.111 & 0.514 & -2.008 \\
\hline $\mathrm{H}$ & 2.535 & -0.187 & -2.534 \\
\hline $\mathrm{H}$ & 2.824 & 1.588 & -2.669 \\
\hline $\mathrm{H}$ & 0.233 & 0.164 & -1.195 \\
\hline $\mathrm{H}$ & 0.391 & 1.940 & -1.297 \\
\hline $\mathrm{H}$ & 0.050 & 0.355 & 1.349 \\
\hline $\mathrm{H}$ & 0.200 & 2.115 & 1.219 \\
\hline $\mathrm{H}$ & -1.742 & 2.664 & -1.219 \\
\hline $\mathrm{H}$ & -3.172 & 2.855 & -0.168 \\
\hline $\mathrm{H}$ & -1.600 & 3.548 & 0.331 \\
\hline $\mathrm{H}$ & -3.490 & 0.341 & -0.231 \\
\hline $\mathrm{H}$ & -2.073 & 0.255 & -1.281 \\
\hline $\mathrm{H}$ & -1.988 & 2.379 & 2.508 \\
\hline $\mathrm{H}$ & -1.998 & 0.604 & 2.641 \\
\hline
\end{tabular}




\begin{tabular}{|c|c|c|c|}
\hline $\mathrm{H}$ & -3.430 & 1.459 & 1.985 \\
\hline \multicolumn{4}{|c|}{36} \\
\hline \multicolumn{4}{|c|}{ Structure 34, Gd complex } \\
\hline $\mathrm{C}$ & -3.723 & -0.035 & 0.851 \\
\hline $\mathrm{P}$ & 2.435 & -0.931 & 0.168 \\
\hline $\mathrm{P}$ & -2.571 & -0.987 & -0.152 \\
\hline $\mathrm{O}$ & 1.394 & -1.966 & 0.433 \\
\hline $\mathrm{C}$ & 3.208 & -0.281 & 1.659 \\
\hline $\mathrm{C}$ & 3.822 & -1.555 & -0.802 \\
\hline O & -1.947 & -2.136 & 0.562 \\
\hline $\mathrm{C}$ & -3.513 & -1.460 & -1.612 \\
\hline $\mathrm{H}$ & -4.577 & -0.673 & 1.156 \\
\hline $\mathrm{H}$ & -4.111 & 0.831 & 0.276 \\
\hline $\mathrm{H}$ & -3.217 & 0.339 & 1.764 \\
\hline $\mathrm{H}$ & 3.731 & -1.096 & 2.200 \\
\hline $\mathrm{H}$ & 2.440 & 0.151 & 2.331 \\
\hline $\mathrm{H}$ & 3.945 & 0.507 & 1.401 \\
\hline $\mathrm{H}$ & 4.302 & -2.404 & -0.275 \\
\hline $\mathrm{H}$ & 4.577 & -0.758 & -0.957 \\
\hline $\mathrm{H}$ & 3.465 & -1.907 & -1.791 \\
\hline $\mathrm{H}$ & -4.345 & -2.135 & -1.325 \\
\hline $\mathrm{H}$ & -2.856 & -1.992 & -2.331 \\
\hline $\mathrm{H}$ & -3.934 & -0.562 & -2.108 \\
\hline $\mathrm{Gd}$ & -0.188 & -3.342 & 1.337 \\
\hline $\mathrm{C}$ & 1.852 & 0.500 & -0.784 \\
\hline $\mathrm{C}$ & 0.851 & 1.425 & -0.057 \\
\hline $\mathrm{C}$ & -0.460 & 0.735 & 0.380 \\
\hline $\mathrm{C}$ & -1.330 & 0.181 & -0.766 \\
\hline $\mathrm{C}$ & 0.542 & 2.646 & -0.945 \\
\hline $\mathrm{H}$ & 2.748 & 1.091 & -1.067 \\
\hline $\mathrm{H}$ & 1.418 & 0.126 & -1.734 \\
\hline $\mathrm{H}$ & 1.350 & 1.810 & 0.864 \\
\hline $\mathrm{H}$ & -0.209 & -0.079 & 1.095 \\
\hline $\mathrm{H}$ & -1.065 & 1.464 & 0.968 \\
\hline $\mathrm{H}$ & -0.705 & -0.362 & -1.503 \\
\hline $\mathrm{H}$ & -1.842 & 1.004 & -1.307 \\
\hline $\mathrm{H}$ & 0.097 & 2.350 & -1.921 \\
\hline $\mathrm{H}$ & 1.463 & 3.231 & -1.169 \\
\hline $\mathrm{H}$ & -0.172 & 3.342 & -0.451 \\
\hline 35 & & & \\
\hline St & ture 34 , & free ligand & global minimum \\
\hline $\mathrm{C}$ & -3.435 & -2.399 & -0.668 \\
\hline $\mathrm{P}$ & 2.091 & 1.266 & 0.921 \\
\hline $\mathrm{P}$ & -1.957 & -1.374 & -0.535 \\
\hline $\mathrm{O}$ & 1.199 & 1.159 & 2.110 \\
\hline $\mathrm{C}$ & 1.400 & 2.245 & -0.426 \\
\hline $\mathrm{C}$ & 3.662 & 2.079 & 1.275 \\
\hline O & -1.526 & -0.785 & -1.835 \\
\hline $\mathrm{C}$ & -2.395 & -0.151 & 0.714 \\
\hline $\mathrm{H}$ & -4.279 & -1.800 & -1.067 \\
\hline $\mathrm{H}$ & -3.721 & -2.799 & 0.326 \\
\hline $\mathrm{H}$ & -3.248 & -3.250 & -1.355 \\
\hline $\mathrm{H}$ & 1.247 & 3.292 & -0.093 \\
\hline $\mathrm{H}$ & 0.420 & 1.831 & -0.740 \\
\hline $\mathrm{H}$ & 2.086 & 2.244 & -1.297 \\
\hline $\mathrm{H}$ & 3.481 & 3.103 & 1.661 \\
\hline $\mathrm{H}$ & 4.279 & 2.148 & 0.356 \\
\hline $\mathrm{H}$ & 4.223 & 1.505 & 2.040 \\
\hline $\mathrm{H}$ & -3.265 & 0.446 & 0.372 \\
\hline $\mathrm{H}$ & -1.545 & 0.538 & 0.892 \\
\hline $\mathrm{H}$ & -2.659 & -0.650 & 1.669 \\
\hline $\mathrm{C}$ & 2.591 & -0.323 & 0.198 \\
\hline $\mathrm{C}$ & 1.429 & -1.149 & -0.399 \\
\hline $\mathrm{C}$ & 0.545 & -1.776 & 0.704 \\
\hline $\mathrm{C}$ & -0.739 & -2.479 & 0.232 \\
\hline $\mathrm{C}$ & 2.008 & -2.244 & -1.317 \\
\hline $\mathrm{H}$ & 3.333 & -0.100 & -0.595 \\
\hline $\mathrm{H}$ & 3.123 & -0.913 & 0.974 \\
\hline $\mathrm{H}$ & 0.811 & -0.473 & -1.030 \\
\hline $\mathrm{H}$ & 1.161 & -2.514 & 1.269 \\
\hline $\mathrm{H}$ & 0.263 & -1.001 & 1.448 \\
\hline $\mathrm{H}$ & -1.228 & -2.965 & 1.103 \\
\hline $\mathrm{H}$ & -0.502 & -3.292 & -0.484 \\
\hline $\mathrm{H}$ & 2.625 & -2.975 & -0.747 \\
\hline $\mathrm{H}$ & 2.658 & -1.811 & -2.110 \\
\hline $\mathrm{H}$ & 1.210 & -2.816 & -1.839 \\
\hline
\end{tabular}




\begin{tabular}{|c|c|c|c|}
\hline $\mathrm{C}$ & 3.700 & -1.387 & -1.524 \\
\hline $\mathrm{P}$ & 2.595 & -0.534 & -0.383 \\
\hline $\mathrm{C}$ & 3.710 & 0.333 & 0.735 \\
\hline 0 & 1.643 & -1.451 & 0.308 \\
\hline $\mathrm{C}$ & -1.281 & 0.649 & -0.534 \\
\hline $\mathrm{C}$ & -0.134 & 1.317 & 0.248 \\
\hline $\mathrm{C}$ & 1.021 & 1.813 & -0.640 \\
\hline $\mathrm{C}$ & 1.804 & 0.735 & -1.412 \\
\hline $\mathrm{C}$ & -3.184 & 0.862 & 1.639 \\
\hline $\mathrm{P}$ & -2.354 & -0.316 & 0.561 \\
\hline $\mathrm{C}$ & -3.651 & -0.953 & -0.514 \\
\hline 0 & -1.594 & -1.375 & 1.284 \\
\hline $\mathrm{Gd}$ & 0.286 & -2.544 & 1.785 \\
\hline $\mathrm{H}$ & 4.290 & -2.152 & -0.980 \\
\hline $\mathrm{H}$ & 3.110 & -1.891 & -2.318 \\
\hline $\mathrm{H}$ & 4.398 & -0.668 & -1.999 \\
\hline $\mathrm{H}$ & 4.340 & -0.398 & 1.282 \\
\hline $\mathrm{H}$ & 4.369 & 1.021 & 0.168 \\
\hline $\mathrm{H}$ & 3.129 & 0.920 & 1.475 \\
\hline $\mathrm{H}$ & -0.878 & -0.050 & -1.295 \\
\hline $\mathrm{H}$ & -1.885 & 1.410 & -1.072 \\
\hline $\mathrm{H}$ & -0.544 & 2.183 & 0.817 \\
\hline $\mathrm{H}$ & 0.274 & 0.621 & 1.014 \\
\hline $\mathrm{H}$ & 1.734 & 2.392 & -0.008 \\
\hline $\mathrm{H}$ & 0.610 & 2.545 & -1.373 \\
\hline $\mathrm{H}$ & 2.600 & 1.227 & -2.010 \\
\hline $\mathrm{H}$ & 1.142 & 0.223 & -2.141 \\
\hline $\mathrm{H}$ & -3.686 & 1.647 & 1.038 \\
\hline $\mathrm{H}$ & -3.946 & 0.341 & 2.254 \\
\hline $\mathrm{H}$ & -2.451 & 1.343 & 2.318 \\
\hline $\mathrm{H}$ & -4.163 & -0.120 & -1.037 \\
\hline $\mathrm{H}$ & -3.215 & -1.637 & -1.270 \\
\hline $\mathrm{H}$ & -4.398 & -1.514 & 0.084 \\
\hline \multicolumn{4}{|c|}{32} \\
\hline \multicolumn{4}{|c|}{ Structure 35, } \\
\hline $\mathrm{C}$ & -2.352 & -1.337 & 0.688 \\
\hline $\mathrm{P}$ & -2.532 & -0.163 & -0.668 \\
\hline $\mathrm{C}$ & -4.317 & 0.057 & -0.812 \\
\hline 0 & -1.884 & -0.601 & -1.937 \\
\hline $\mathrm{C}$ & 2.002 & 1.365 & -0.330 \\
\hline $\mathrm{C}$ & 0.527 & 1.318 & -0.762 \\
\hline $\mathrm{C}$ & -0.479 & 1.446 & 0.396 \\
\hline $\mathrm{C}$ & -1.953 & 1.438 & -0.040 \\
\hline $\mathrm{C}$ & 2.339 & -1.507 & -0.487 \\
\hline $\mathrm{P}$ & 2.547 & -0.090 & 0.607 \\
\hline $\mathrm{C}$ & 4.337 & 0.116 & 0.708 \\
\hline $\mathrm{O}$ & 1.893 & -0.251 & 1.937 \\
\hline $\mathrm{H}$ & -2.852 & -2.293 & 0.431 \\
\hline $\mathrm{H}$ & -1.279 & -1.542 & 0.880 \\
\hline $\mathrm{H}$ & -2.811 & -0.932 & 1.614 \\
\hline $\mathrm{H}$ & -4.793 & -0.895 & -1.126 \\
\hline $\mathrm{H}$ & -4.749 & 0.368 & 0.161 \\
\hline $\mathrm{H}$ & -4.544 & 0.835 & -1.569 \\
\hline $\mathrm{H}$ & 2.193 & 2.271 & 0.284 \\
\hline $\mathrm{H}$ & 2.646 & 1.449 & -1.230 \\
\hline $\mathrm{H}$ & 0.344 & 2.152 & -1.479 \\
\hline $\mathrm{H}$ & 0.343 & 0.382 & -1.332 \\
\hline $\mathrm{H}$ & -0.276 & 2.402 & 0.932 \\
\hline $\mathrm{H}$ & -0.314 & 0.639 & 1.143 \\
\hline $\mathrm{H}$ & -2.126 & 2.206 & -0.823 \\
\hline $\mathrm{H}$ & -2.594 & 1.714 & 0.824 \\
\hline $\mathrm{H}$ & 2.804 & -1.305 & -1.474 \\
\hline $\mathrm{H}$ & 2.819 & -2.402 & -0.043 \\
\hline $\mathrm{H}$ & 1.262 & -1.723 & -0.636 \\
\hline $\mathrm{H}$ & 4.774 & 0.217 & -0.306 \\
\hline $\mathrm{H}$ & 4.582 & 1.024 & 1.295 \\
\hline $\mathrm{H}$ & 4.793 & -0.764 & 1.207 \\
\hline \multicolumn{4}{|c|}{31} \\
\hline St & Eure & Gd complex & \\
\hline $\mathrm{P}$ & -1.858 & -0.442 & 0.574 \\
\hline 0 & -1.090 & -1.691 & 0.833 \\
\hline $\mathrm{C}$ & -3.415 & -0.807 & -0.261 \\
\hline $\mathrm{C}$ & -2.358 & 0.405 & 2.083 \\
\hline $\mathrm{P}$ & 1.698 & -0.322 & 0.024 \\
\hline 0 & 1.309 & -1.748 & -0.149 \\
\hline $\mathrm{C}$ & 1.889 & 0.154 & 1.753 \\
\hline $\mathrm{C}$ & 3.345 & 0.017 & -0.630 \\
\hline $\mathrm{H}$ & -3.948 & -1.615 & 0.281 \\
\hline $\mathrm{H}$ & -3.219 & -1.149 & -1.298 \\
\hline
\end{tabular}




\begin{tabular}{|c|c|c|c|}
\hline $\mathrm{H}$ & -4.077 & 0.082 & -0.292 \\
\hline $\mathrm{H}$ & -2.965 & -0.274 & 2.716 \\
\hline $\mathrm{H}$ & -2.961 & 1.304 & 1.843 \\
\hline $\mathrm{H}$ & -1.462 & 0.720 & 2.657 \\
\hline $\mathrm{H}$ & 2.688 & -0.451 & 2.228 \\
\hline $\mathrm{H}$ & 0.943 & -0.018 & 2.304 \\
\hline $\mathrm{H}$ & 2.157 & 1.227 & 1.830 \\
\hline $\mathrm{H}$ & 4.077 & -0.683 & -0.177 \\
\hline $\mathrm{H}$ & 3.663 & 1.053 & -0.391 \\
\hline $\mathrm{H}$ & 3.361 & -0.128 & -1.729 \\
\hline Gd & 0.097 & -3.588 & 0.379 \\
\hline $\mathrm{C}$ & $-1 \cdot 220$ & 2.779 & -1.809 \\
\hline $\mathrm{C}$ & 0.144 & 2.803 & -2.069 \\
\hline C & 0.972 & 1.835 & -1.505 \\
\hline $\mathrm{C}$ & 0.457 & 0.825 & -0.677 \\
\hline C & -0.936 & 0.786 & -0.429 \\
\hline $\mathrm{C}$ & -1.751 & 1.781 & -0.995 \\
\hline $\mathrm{H}$ & -1.880 & 3.548 & -2.246 \\
\hline $\mathrm{H}$ & 0.570 & 3.588 & -2.716 \\
\hline $\mathrm{H}$ & 2.047 & 1.896 & -1.733 \\
\hline $\mathrm{H}$ & -2.836 & 1.802 & -0.808 \\
\hline \multicolumn{4}{|l|}{30} \\
\hline \multicolumn{4}{|c|}{ Structure 36, } \\
\hline $\mathrm{P}$ & 2.055 & -0.572 & 0.507 \\
\hline 0 & 3.478 & -0.262 & 0.179 \\
\hline C & 1.700 & -2.301 & 0.146 \\
\hline C & 1.739 & -0.248 & 2.251 \\
\hline P & -1.645 & -0.488 & 0.494 \\
\hline 0 & -0.979 & -1.447 & 1.416 \\
\hline C & -2.747 & -1.323 & -0.664 \\
\hline $\mathrm{C}$ & -2.710 & 0.668 & 1.378 \\
\hline $\mathrm{H}$ & 2.324 & -2.956 & 0.787 \\
\hline $\mathrm{H}$ & 1.944 & -2.517 & -0.914 \\
\hline $\mathrm{H}$ & 0.639 & -2.560 & 0.311 \\
\hline $\mathrm{H}$ & 2.363 & -0.917 & 2.877 \\
\hline $\mathrm{H}$ & 0.680 & -0.400 & 2.526 \\
\hline $\mathrm{H}$ & 2.007 & 0.802 & 2.489 \\
\hline $\mathrm{H}$ & -3.478 & -1.945 & -0.107 \\
\hline $\mathrm{H}$ & -2.161 & -1.983 & -1.335 \\
\hline $\mathrm{H}$ & -3.307 & -0.592 & -1.281 \\
\hline $\mathrm{H}$ & -3.439 & 0.110 & 2.000 \\
\hline $\mathrm{H}$ & -3.270 & 1.314 & 0.674 \\
\hline $\mathrm{H}$ & -2.098 & 1.312 & 2.043 \\
\hline $\mathrm{C}$ & 0.971 & 2.225 & -2.200 \\
\hline $\mathrm{C}$ & -0.418 & 2.259 & -2.208 \\
\hline $\mathrm{C}$ & -1.131 & 1.410 & -1.366 \\
\hline C & -0.479 & 0.511 & -0.502 \\
\hline C & 0.933 & 0.476 & -0.494 \\
\hline C & 1.634 & 1.342 & -1.352 \\
\hline $\mathrm{H}$ & 1.546 & 2.893 & -2.863 \\
\hline $\mathrm{H}$ & -0.950 & 2.955 & -2.877 \\
\hline $\mathrm{H}$ & -2.231 & 1.469 & -1.404 \\
\hline $\mathrm{H}$ & 2.736 & 1.345 & -1.375 \\
\hline \multicolumn{4}{|l|}{36} \\
\hline \multicolumn{4}{|c|}{ Str } \\
\hline $\mathrm{C}$ & -3.570 & 0.807 & 1.363 \\
\hline $\mathrm{P}$ & 2.420 & -0.523 & 0.103 \\
\hline P & -2.602 & -0.272 & 0.296 \\
\hline 0 & 1.320 & -1.445 & 0.510 \\
\hline C & 3.332 & 0.141 & 1.509 \\
\hline $\mathrm{C}$ & 3.690 & -1.371 & -0.857 \\
\hline 0 & -1.982 & -1.430 & 0.999 \\
\hline $\mathrm{C}$ & -3.727 & -0.740 & -1.030 \\
\hline $\mathrm{H}$ & -4.428 & 0.248 & 1.790 \\
\hline $\mathrm{H}$ & -3.958 & 1.672 & 0.787 \\
\hline $\mathrm{H}$ & -2.943 & 1.183 & 2.197 \\
\hline $\mathrm{H}$ & 3.766 & -0.690 & 2.102 \\
\hline $\mathrm{H}$ & 2.653 & 0.721 & 2.167 \\
\hline $\mathrm{H}$ & 4.155 & 0.800 & 1.170 \\
\hline $\mathrm{H}$ & 4.031 & -2.275 & -0.313 \\
\hline $\mathrm{H}$ & 4.567 & -0.713 & -1.023 \\
\hline $\mathrm{H}$ & 3.280 & -1.683 & -1.839 \\
\hline $\mathrm{H}$ & -4.567 & -1.337 & -0.622 \\
\hline $\mathrm{H}$ & -3.190 & -1.350 & -1.785 \\
\hline $\mathrm{H}$ & -4.137 & 0.164 & -1.526 \\
\hline $\mathrm{Gd}$ & -0.217 & -2.709 & 1.632 \\
\hline $\mathrm{C}$ & 3.022 & 1.674 & -1.520 \\
\hline $\mathrm{C}$ & 1.841 & 0.892 & -0.910 \\
\hline $\mathrm{C}$ & 0.946 & 1.881 & -0.123 \\
\hline
\end{tabular}




\begin{tabular}{|c|c|c|c|}
\hline $\mathrm{C}$ & -0.335 & 1.312 & 0.514 \\
\hline $\mathrm{C}$ & -1.356 & 0.771 & -0.505 \\
\hline $\mathrm{H}$ & -0.850 & 0.146 & -1.269 \\
\hline $\mathrm{H}$ & -1.856 & 1.605 & -1.040 \\
\hline $\mathrm{H}$ & -0.821 & 2.117 & 1.113 \\
\hline $\mathrm{H}$ & -0.057 & 0.524 & 1.247 \\
\hline $\mathrm{H}$ & 0.647 & 2.709 & -0.806 \\
\hline $\mathrm{H}$ & 1.556 & 2.361 & 0.677 \\
\hline $\mathrm{H}$ & 1.249 & 0.484 & -1.756 \\
\hline $\mathrm{H}$ & 3.641 & 1.044 & -2.197 \\
\hline $\mathrm{H}$ & 3.697 & 2.098 & -0.744 \\
\hline $\mathrm{H}$ & 2.664 & 2.530 & -2.136 \\
\hline \multicolumn{4}{|l|}{35} \\
\hline Str & cure 37, & free ligand & global minimur \\
\hline $\mathrm{C}$ & -2.410 & 0.246 & 1.645 \\
\hline $\mathrm{P}$ & 2.492 & 0.097 & 0.938 \\
\hline $\mathrm{P}$ & -2.477 & -0.451 & -0.016 \\
\hline O & 1.957 & 1.288 & 1.659 \\
\hline $\mathrm{C}$ & 4.275 & -0.112 & 1.121 \\
\hline $\mathrm{C}$ & 1.845 & -1.473 & 1.544 \\
\hline 0 & -1.894 & -1.819 & -0.122 \\
\hline $\mathrm{C}$ & -4.229 & -0.382 & -0.439 \\
\hline $\mathrm{H}$ & -3.010 & -0.375 & 2.341 \\
\hline $\mathrm{H}$ & -2.815 & 1.279 & 1.648 \\
\hline $\mathrm{H}$ & -1.363 & 0.271 & 2.010 \\
\hline $\mathrm{H}$ & 4.531 & -0.264 & 2.189 \\
\hline $\mathrm{H}$ & 4.808 & 0.789 & 0.757 \\
\hline $\mathrm{H}$ & 4.624 & -0.992 & 0.543 \\
\hline $\mathrm{H}$ & 2.151 & -1.628 & 2.599 \\
\hline $\mathrm{H}$ & 2.233 & -2.314 & 0.933 \\
\hline $\mathrm{H}$ & 0.737 & -1.477 & 1.499 \\
\hline $\mathrm{H}$ & -4.808 & -1.049 & 0.231 \\
\hline $\mathrm{H}$ & -4.380 & -0.712 & -1.487 \\
\hline $\mathrm{H}$ & -4.614 & 0.653 & -0.331 \\
\hline $\mathrm{C}$ & 2.774 & 1.390 & -1.505 \\
\hline C & 2.202 & 0.108 & -0.869 \\
\hline $\mathrm{C}$ & 0.712 & -0.080 & -1.237 \\
\hline $\mathrm{C}$ & -0.239 & 1.060 & -0.824 \\
\hline C & -1.723 & 0.779 & -1.118 \\
\hline $\mathrm{H}$ & -1.849 & 0.439 & -2.167 \\
\hline $\mathrm{H}$ & -2.303 & 1.721 & -1.017 \\
\hline $\mathrm{H}$ & -0.118 & 1.299 & 0.255 \\
\hline $\mathrm{H}$ & 0.053 & 1.983 & -1.375 \\
\hline $\mathrm{H}$ & 0.358 & -1.039 & -0.802 \\
\hline $\mathrm{H}$ & 0.636 & -0.213 & -2.341 \\
\hline $\mathrm{H}$ & 2.755 & -0.753 & -1.304 \\
\hline $\mathrm{H}$ & 3.879 & 1.453 & -1.388 \\
\hline $\mathrm{H}$ & 2.344 & 2.314 & -1.059 \\
\hline $\mathrm{H}$ & 2.569 & 1.426 & -2.599 \\
\hline \multicolumn{4}{|l|}{34} \\
\hline Str & cure 38, & Gd complex & \\
\hline C & -0.037 & 2.379 & -1.652 \\
\hline $\mathrm{C}$ & -1.268 & 1.895 & -1.199 \\
\hline $\mathrm{C}$ & -1.302 & 0.945 & -0.178 \\
\hline C & -0.107 & 0.525 & 0.411 \\
\hline C & 1.131 & 0.941 & -0.085 \\
\hline $\mathrm{C}$ & 1.157 & 1.889 & -1.115 \\
\hline $\mathrm{C}$ & 2.377 & 0.277 & 0.447 \\
\hline $\mathrm{P}$ & -2.777 & 0.015 & 0.292 \\
\hline $\mathrm{P}$ & 2.414 & -1.481 & -0.023 \\
\hline 0 & -2.365 & -1.360 & 0.703 \\
\hline $\mathrm{C}$ & 2.539 & -1.481 & -1.819 \\
\hline C & 4.019 & -2.065 & 0.551 \\
\hline $\mathrm{C}$ & -3.598 & 0.952 & 1.588 \\
\hline C & -3.933 & 0.019 & -1.088 \\
\hline 0 & 1.264 & -2.281 & 0.495 \\
\hline $\mathrm{Gd}$ & -0.858 & -3.073 & 0.902 \\
\hline $\mathrm{H}$ & -0.007 & 3.116 & -2.472 \\
\hline $\mathrm{H}$ & -2.195 & 2.236 & -1.686 \\
\hline $\mathrm{H}$ & -0.129 & -0.216 & 1.224 \\
\hline $\mathrm{H}$ & 2.121 & 2.237 & -1.524 \\
\hline $\mathrm{H}$ & 2.413 & 0.354 & 1.553 \\
\hline $\mathrm{H}$ & 3.289 & 0.772 & 0.054 \\
\hline $\mathrm{H}$ & 3.393 & -0.854 & -2.148 \\
\hline $\mathrm{H}$ & 2.692 & -2.516 & -2.188 \\
\hline $\mathrm{H}$ & 1.606 & -1.081 & -2.266 \\
\hline $\mathrm{H}$ & 4.832 & -1.440 & 0.129 \\
\hline $\mathrm{H}$ & 4.067 & -2.015 & 1.658 \\
\hline $\mathrm{H}$ & 4.175 & -3.116 & 0.235 \\
\hline
\end{tabular}




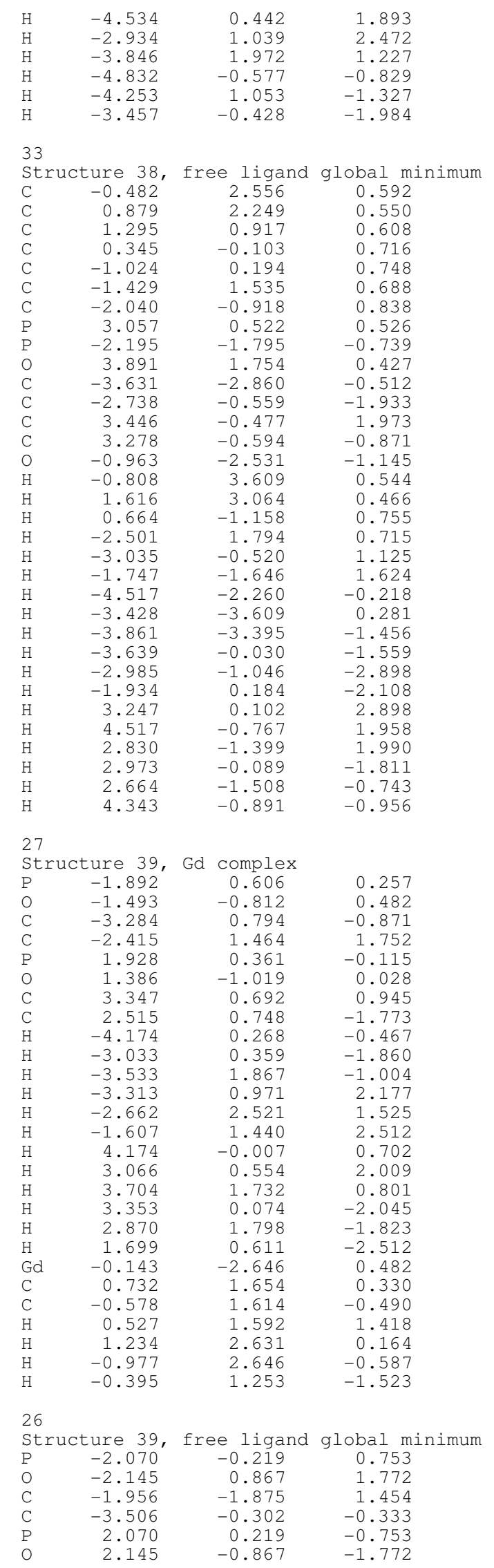




\begin{tabular}{|c|c|c|c|}
\hline $\mathrm{C}$ & 3.506 & 0.302 & 0.333 \\
\hline $\mathrm{C}$ & 1.956 & 1.875 & -1.454 \\
\hline $\mathrm{H}$ & -2.863 & -2.097 & 2.053 \\
\hline $\mathrm{H}$ & -1.069 & -1.952 & 2.115 \\
\hline $\mathrm{H}$ & -1.868 & -2.631 & 0.647 \\
\hline $\mathrm{H}$ & -4.424 & -0.486 & 0.263 \\
\hline $\mathrm{H}$ & -3.388 & -1.124 & -1.068 \\
\hline $\mathrm{H}$ & -3.624 & 0.654 & -0.882 \\
\hline $\mathrm{H}$ & 4.424 & 0.486 & -0.263 \\
\hline $\mathrm{H}$ & 3.624 & -0.654 & 0.882 \\
\hline $\mathrm{H}$ & 3.388 & 1.124 & 1.068 \\
\hline $\mathrm{H}$ & 2.863 & 2.097 & -2.053 \\
\hline $\mathrm{H}$ & 1.868 & 2.631 & -0.647 \\
\hline $\mathrm{H}$ & 1.069 & 1.952 & -2.115 \\
\hline $\mathrm{C}$ & 0.666 & 0.056 & 0.380 \\
\hline $\mathrm{C}$ & -0.666 & -0.056 & -0.380 \\
\hline $\mathrm{H}$ & 0.836 & -0.848 & 1.003 \\
\hline $\mathrm{H}$ & 0.661 & 0.933 & 1.062 \\
\hline $\mathrm{H}$ & -0.836 & 0.848 & -1.003 \\
\hline $\mathrm{H}$ & -0.661 & -0.933 & -1.062 \\
\hline \multicolumn{4}{|c|}{30} \\
\hline \multicolumn{4}{|c|}{ Structure 40, Gd complex } \\
\hline $\mathrm{P}$ & 2.126 & 0.104 & 0.284 \\
\hline O & 1.363 & -1.138 & 0.590 \\
\hline $\mathrm{C}$ & 2.723 & 0.994 & 1.730 \\
\hline $\mathrm{C}$ & 3.621 & -0.213 & -0.675 \\
\hline $\mathrm{P}$ & -2.036 & 0.305 & -0.225 \\
\hline O & -1.406 & -1.024 & -0.461 \\
\hline $\mathrm{C}$ & -2.542 & 1.172 & -1.719 \\
\hline $\mathrm{C}$ & -3.552 & 0.196 & 0.746 \\
\hline $\mathrm{H}$ & 3.423 & 0.355 & 2.306 \\
\hline $\mathrm{H}$ & 1.874 & 1.269 & 2.389 \\
\hline $\mathrm{H}$ & 3.253 & 1.918 & 1.422 \\
\hline $\mathrm{H}$ & 4.293 & -0.894 & -0.115 \\
\hline $\mathrm{H}$ & 4.160 & 0.735 & -0.878 \\
\hline $\mathrm{H}$ & 3.357 & -0.690 & -1.641 \\
\hline $\mathrm{H}$ & -3.306 & 0.579 & -2.261 \\
\hline $\mathrm{H}$ & -1.672 & 1.323 & -2.389 \\
\hline $\mathrm{H}$ & -2.973 & 2.161 & -1.463 \\
\hline $\mathrm{H}$ & -4.293 & -0.441 & 0.221 \\
\hline $\mathrm{H}$ & -3.992 & 1.203 & 0.896 \\
\hline $\mathrm{H}$ & -3.337 & -0.253 & 1.737 \\
\hline $\mathrm{Gd}$ & -0.110 & -2.806 & 0.111 \\
\hline $\mathrm{C}$ & 1.193 & 1.263 & -0.750 \\
\hline $\mathrm{C}$ & 0.144 & 2.121 & -0.022 \\
\hline $\mathrm{C}$ & -0.986 & 1.416 & 0.748 \\
\hline $\mathrm{H}$ & 1.926 & 1.939 & -1.239 \\
\hline $\mathrm{H}$ & 0.717 & 0.682 & -1.568 \\
\hline $\mathrm{H}$ & 0.676 & 2.794 & 0.689 \\
\hline $\mathrm{H}$ & -0.318 & 2.806 & -0.770 \\
\hline $\mathrm{H}$ & -1.645 & 2.189 & 1.198 \\
\hline $\mathrm{H}$ & -0.570 & 0.835 & 1.597 \\
\hline \multicolumn{4}{|c|}{29} \\
\hline St & Eure 40 , & free ligand & global minimum \\
\hline $\mathrm{P}$ & -1.563 & 0.908 & -1.885 \\
\hline O & -1.271 & 2.246 & -2.475 \\
\hline $\mathrm{C}$ & -2.831 & 0.933 & -0.604 \\
\hline $\mathrm{C}$ & -2.173 & -0.298 & -3.078 \\
\hline $\mathrm{P}$ & 1.519 & -1.018 & 1.643 \\
\hline 0 & 0.796 & -2.163 & 1.021 \\
\hline $\mathrm{C}$ & 0.684 & -0.321 & 3.080 \\
\hline $\mathrm{C}$ & 3.157 & -1.448 & 2.263 \\
\hline $\mathrm{H}$ & -3.793 & 1.284 & -1.029 \\
\hline $\mathrm{H}$ & -2.535 & 1.619 & 0.216 \\
\hline $\mathrm{H}$ & -2.976 & -0.084 & -0.184 \\
\hline $\mathrm{H}$ & -3.115 & 0.065 & -3.538 \\
\hline $\mathrm{H}$ & -2.370 & -1.269 & -2.580 \\
\hline $\mathrm{H}$ & -1.422 & -0.454 & -3.880 \\
\hline $\mathrm{H}$ & 0.603 & -1.086 & 3.880 \\
\hline $\mathrm{H}$ & -0.338 & 0.012 & 2.807 \\
\hline $\mathrm{H}$ & 1.250 & 0.548 & 3.474 \\
\hline $\mathrm{H}$ & 3.076 & -2.246 & 3.030 \\
\hline $\mathrm{H}$ & 3.643 & -0.562 & 2.720 \\
\hline $\mathrm{H}$ & 3.793 & -1.816 & 1.432 \\
\hline $\mathrm{C}$ & -0.124 & 0.099 & -1.140 \\
\hline $\mathrm{C}$ & 0.559 & 0.988 & -0.086 \\
\hline $\mathrm{C}$ & 1.832 & 0.371 & 0.520 \\
\hline $\mathrm{H}$ & -0.461 & -0.859 & -0.695 \\
\hline $\mathrm{H}$ & 0.594 & -0.149 & -1.950 \\
\hline
\end{tabular}

S-63 


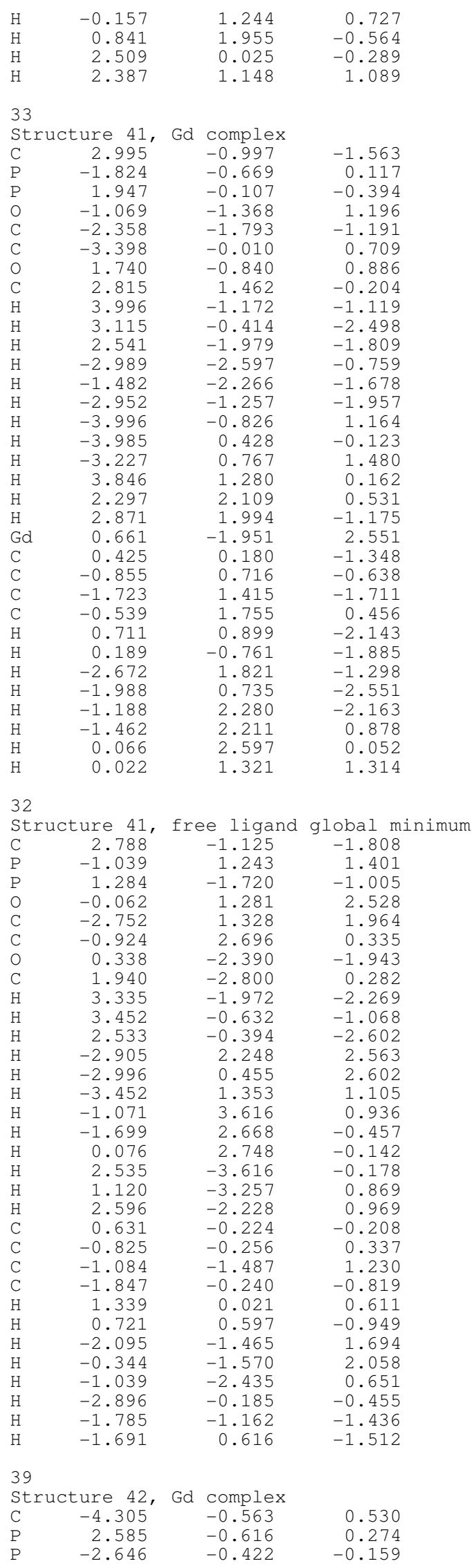




\begin{tabular}{|c|c|c|c|}
\hline 0 & 1.744 & -1.801 & -0.060 \\
\hline C & 2.776 & -0.379 & 2.055 \\
\hline C & 4.310 & -0.816 & -0.222 \\
\hline 0 & -1.865 & -1.691 & -0.123 \\
\hline C & -2.933 & 0.203 & -1.826 \\
\hline $\mathrm{H}$ & -4.892 & -1.310 & -0.044 \\
\hline $\mathrm{H}$ & -4.827 & 0.414 & 0.484 \\
\hline $\mathrm{H}$ & -4.251 & -0.891 & 1.588 \\
\hline $\mathrm{H}$ & 3.158 & -1.312 & 2.517 \\
\hline $\mathrm{H}$ & 1.806 & -0.132 & 2.529 \\
\hline $\mathrm{H}$ & 3.497 & 0.435 & 2.269 \\
\hline $\mathrm{H}$ & 4.764 & -1.665 & 0.328 \\
\hline $\mathrm{H}$ & 4.892 & 0.101 & 0.002 \\
\hline $\mathrm{H}$ & 4.378 & -1.031 & -1.307 \\
\hline $\mathrm{H}$ & -3.500 & -0.545 & -2.416 \\
\hline $\mathrm{H}$ & -1.970 & 0.399 & -2.338 \\
\hline $\mathrm{H}$ & -3.516 & 1.146 & -1.791 \\
\hline $\mathrm{Gd}$ & -0.098 & -3.116 & -0.286 \\
\hline $\mathrm{C}$ & 2.407 & 0.979 & -1.961 \\
\hline C & 1.941 & 0.935 & -0.487 \\
\hline $\mathrm{C}$ & 0.389 & 0.942 & -0.499 \\
\hline C & -0.339 & 0.833 & 0.853 \\
\hline C & -1.873 & 0.935 & 0.766 \\
\hline C & 2.494 & 2.178 & 0.236 \\
\hline $\mathrm{H}$ & 3.512 & 1.069 & -2.054 \\
\hline $\mathrm{H}$ & 1.978 & 1.855 & -2.496 \\
\hline $\mathrm{H}$ & 2.098 & 0.073 & -2.529 \\
\hline $\mathrm{H}$ & 0.047 & 1.879 & -0.997 \\
\hline $\mathrm{H}$ & 0.045 & 0.113 & -1.157 \\
\hline $\mathrm{H}$ & -0.079 & -0.127 & 1.350 \\
\hline $\mathrm{H}$ & 0.023 & 1.642 & 1.526 \\
\hline $\mathrm{H}$ & -2.177 & 1.902 & 0.313 \\
\hline $\mathrm{H}$ & -2.292 & 0.922 & 1.794 \\
\hline $\mathrm{H}$ & 2.186 & 2.228 & 1.302 \\
\hline $\mathrm{H}$ & 2.134 & 3.116 & -0.243 \\
\hline $\mathrm{H}$ & 3.606 & 2.216 & 0.213 \\
\hline \multicolumn{4}{|c|}{38} \\
\hline \multicolumn{4}{|c|}{ Structure 42, } \\
\hline $\mathrm{C}$ & 1.112 & 1.994 & -2.405 \\
\hline $\mathrm{P}$ & -2.362 & -0.636 & -0.013 \\
\hline $\mathrm{P}$ & 2.245 & 1.036 & -1.381 \\
\hline 0 & -2.648 & 0.815 & -0.210 \\
\hline C & -3.861 & -1.586 & 0.322 \\
\hline $\mathrm{C}$ & -1.734 & -1.432 & -1.507 \\
\hline O & 2.244 & -0.424 & -1.680 \\
\hline C & 3.843 & 1.825 & -1.658 \\
\hline $\mathrm{H}$ & 1.410 & 1.919 & -3.471 \\
\hline $\mathrm{H}$ & 1.128 & 3.062 & -2.105 \\
\hline $\mathrm{H}$ & 0.078 & 1.608 & -2.301 \\
\hline $\mathrm{H}$ & -4.612 & -1.392 & -0.471 \\
\hline $\mathrm{H}$ & -4.298 & -1.288 & 1.296 \\
\hline $\mathrm{H}$ & -3.648 & -2.673 & 0.338 \\
\hline $\mathrm{H}$ & -2.474 & -1.330 & -2.327 \\
\hline $\mathrm{H}$ & -1.548 & -2.511 & -1.335 \\
\hline $\mathrm{H}$ & -0.789 & -0.952 & -1.833 \\
\hline $\mathrm{H}$ & 4.153 & 1.697 & -2.715 \\
\hline $\mathrm{H}$ & 4.612 & 1.366 & -1.004 \\
\hline $\mathrm{H}$ & 3.784 & 2.910 & -1.433 \\
\hline $\mathrm{C}$ & -1.083 & -2.482 & 1.634 \\
\hline $\mathrm{C}$ & -1.167 & -0.965 & 1.354 \\
\hline $\mathrm{C}$ & 0.254 & -0.468 & 0.983 \\
\hline C & 0.437 & 1.049 & 0.781 \\
\hline $\mathrm{C}$ & 1.854 & 1.456 & 0.341 \\
\hline $\mathrm{C}$ & -1.671 & -0.263 & 2.633 \\
\hline $\mathrm{H}$ & -2.057 & -2.910 & 1.961 \\
\hline $\mathrm{H}$ & -0.359 & -2.702 & 2.451 \\
\hline $\mathrm{H}$ & -0.745 & -3.062 & 0.747 \\
\hline $\mathrm{H}$ & 0.958 & -0.788 & 1.785 \\
\hline $\mathrm{H}$ & 0.589 & -1.006 & 0.070 \\
\hline $\mathrm{H}$ & -0.298 & 1.440 & 0.046 \\
\hline $\mathrm{H}$ & 0.214 & 1.565 & 1.743 \\
\hline $\mathrm{H}$ & 2.612 & 0.984 & 1.001 \\
\hline $\mathrm{H}$ & 1.973 & 2.555 & 0.454 \\
\hline $\mathrm{H}$ & -1.826 & 0.830 & 2.488 \\
\hline $\mathrm{H}$ & -0.948 & -0.379 & 3.471 \\
\hline $\mathrm{H}$ & -2.639 & -0.682 & 2.987 \\
\hline 7 & & & \\
\hline St & Eure 43 , & Gd complex & \\
\hline $\mathrm{C}$ & 0.766 & 2.853 & -1.724 \\
\hline
\end{tabular}




\begin{tabular}{|c|c|c|c|}
\hline & & & \\
\hline $\mathrm{C}$ & -0.594 & 2.723 & -1.987 \\
\hline $\mathrm{C}$ & -1.345 & 1.779 & -1.291 \\
\hline C & -0.751 & 0.944 & -0.331 \\
\hline C & 0.636 & 1.047 & -0.084 \\
\hline $\mathrm{C}$ & 1.369 & 2.029 & -0.777 \\
\hline C & 1.398 & 0.243 & 0.949 \\
\hline $\mathrm{P}$ & -1.840 & -0.231 & 0.538 \\
\hline $\mathrm{P}$ & 2.316 & -1.168 & 0.268 \\
\hline 0 & -1.224 & -1.584 & 0.642 \\
\hline C & 3.403 & -0.588 & -1.043 \\
\hline $\mathrm{C}$ & 3.447 & -1.601 & 1.605 \\
\hline C & -2.232 & 0.517 & 2.130 \\
\hline C & -3.456 & -0.296 & -0.261 \\
\hline 0 & 1.440 & -2.303 & -0.134 \\
\hline $\mathrm{Gd}$ & -0.406 & -3.609 & -0.009 \\
\hline $\mathrm{H}$ & 1.363 & 3.609 & -2.261 \\
\hline $\mathrm{H}$ & -1.077 & 3.372 & -2.737 \\
\hline $\mathrm{H}$ & -2.421 & 1.713 & -1.517 \\
\hline $\mathrm{H}$ & 2.443 & 2.165 & -0.570 \\
\hline $\mathrm{H}$ & 0.728 & -0.133 & 1.747 \\
\hline $\mathrm{H}$ & 2.125 & 0.906 & 1.462 \\
\hline $\mathrm{H}$ & 4.038 & 0.246 & -0.681 \\
\hline $\mathrm{H}$ & 2.806 & -0.240 & -1.910 \\
\hline $\mathrm{H}$ & 4.062 & -1.414 & -1.379 \\
\hline $\mathrm{H}$ & 4.085 & -0.733 & 1.870 \\
\hline $\mathrm{H}$ & 4.100 & -2.441 & 1.291 \\
\hline $\mathrm{H}$ & 2.873 & -1.912 & 2.501 \\
\hline $\mathrm{H}$ & -1.312 & 0.635 & 2.737 \\
\hline $\mathrm{H}$ & -2.944 & -0.124 & 2.687 \\
\hline $\mathrm{H}$ & -2.689 & 1.517 & 1.982 \\
\hline $\mathrm{H}$ & -3.349 & -0.614 & -1.318 \\
\hline $\mathrm{H}$ & -3.959 & 0.692 & -0.223 \\
\hline $\mathrm{H}$ & -4.100 & -1.033 & 0.261 \\
\hline 33 & & & \\
\hline St & cure 43, & free ligand & global minimu \\
\hline $\mathrm{C}$ & -1.848 & 2.823 & -0.979 \\
\hline $\mathrm{C}$ & -0.505 & 3.163 & -1.119 \\
\hline $\mathrm{C}$ & 0.481 & 2.272 & -0.701 \\
\hline $\mathrm{C}$ & 0.143 & 1.028 & -0.140 \\
\hline $\mathrm{C}$ & -1.215 & 0.680 & 0.007 \\
\hline C & -2.195 & 1.596 & -0.418 \\
\hline $\mathrm{C}$ & -1.726 & -0.600 & 0.631 \\
\hline $\mathrm{P}$ & 1.555 & -0.028 & 0.330 \\
\hline $\mathrm{P}$ & -2.306 & -1.784 & -0.614 \\
\hline O & 1.149 & $-1 \cdot 388$ & 0.781 \\
\hline C & -0.937 & -2.147 & -1.726 \\
\hline $\mathrm{C}$ & -2.516 & -3.312 & 0.321 \\
\hline C & 2.675 & -0.091 & -1.082 \\
\hline $\mathrm{C}$ & 2.473 & 0.875 & 1.591 \\
\hline O & -3.544 & -1.345 & -1.320 \\
\hline $\mathrm{H}$ & -2.635 & 3.521 & -1.310 \\
\hline $\mathrm{H}$ & -0.225 & 4.134 & -1.560 \\
\hline $\begin{array}{l}11 \\
\mathrm{H}\end{array}$ & 1.534 & 2.574 & -0.822 \\
\hline $\mathrm{H}$ & -3.264 & 1.344 & -0.316 \\
\hline $\mathrm{H}$ & -2.582 & -0.370 & 1.299 \\
\hline $\mathrm{H}$ & -0.977 & -1.082 & 1.283 \\
\hline $\mathrm{H}$ & -0.075 & -2.562 & -1.166 \\
\hline $\mathrm{H}$ & -0.616 & -1.225 & -2.253 \\
\hline $\mathrm{H}$ & -1.254 & -2.893 & -2.483 \\
\hline $\mathrm{H}$ & -1.566 & -3.594 & 0.820 \\
\hline $\mathrm{H}$ & -2.820 & -4.134 & -0.358 \\
\hline $\mathrm{H}$ & -3.300 & -3.180 & 1.094 \\
\hline $\mathrm{H}$ & 2.151 & -0.512 & -1.964 \\
\hline $\mathrm{H}$ & 3.544 & -0.739 & -0.845 \\
\hline $\mathrm{H}$ & 3.052 & 0.919 & -1.338 \\
\hline $\mathrm{H}$ & 1.833 & 1.035 & 2.483 \\
\hline $\mathrm{H}$ & 2.803 & 1.861 & 1.207 \\
\hline $\mathrm{H}$ & 3.369 & 0.296 & 1.896 \\
\hline 33 & & & \\
\hline St & ture 44, & Gd complex & \\
\hline $\mathrm{C}$ & 3.347 & 0.844 & -1.118 \\
\hline $\mathrm{P}$ & -1.998 & -1.075 & -0.123 \\
\hline $\mathrm{P}$ & 2.345 & -0.218 & -0.065 \\
\hline 0 & -1.151 & -2.016 & -0.911 \\
\hline C & -3.756 & -1.178 & -0.513 \\
\hline C & -1.959 & -1.394 & 1.650 \\
\hline 0 & 2.010 & -1.532 & -0.683 \\
\hline C & 3.291 & -0.353 & 1.463 \\
\hline $\mathrm{H}$ & 4.330 & 0.367 & -1.311 \\
\hline
\end{tabular}




\begin{tabular}{|c|c|c|c|}
\hline $\mathrm{H}$ & 3.516 & 1.825 & -0.630 \\
\hline $\mathrm{H}$ & 2.839 & 1.006 & -2.090 \\
\hline $\mathrm{H}$ & -4.132 & -2.201 & -0.303 \\
\hline $\mathrm{H}$ & -3.922 & -0.952 & -1.586 \\
\hline $\mathrm{H}$ & -4.330 & -0.453 & 0.100 \\
\hline $\mathrm{H}$ & -2.396 & -2.391 & 1.864 \\
\hline $\mathrm{H}$ & -2.544 & -0.625 & 2.196 \\
\hline $\mathrm{H}$ & -0.916 & -1.386 & 2.025 \\
\hline $\mathrm{H}$ & 4.255 & -0.867 & 1.268 \\
\hline $\mathrm{H}$ & 2.720 & -0.939 & 2.212 \\
\hline $\mathrm{H}$ & 3.500 & 0.653 & 1.879 \\
\hline $\mathrm{Gd}$ & 0.700 & -3.132 & -1.629 \\
\hline $\mathrm{C}$ & 0.872 & 0.731 & 0.404 \\
\hline $\mathrm{C}$ & -0.118 & 0.941 & -0.778 \\
\hline $\mathrm{C}$ & -1.589 & 0.677 & -0.381 \\
\hline $\mathrm{C}$ & 0.010 & 2.380 & -1.317 \\
\hline $\mathrm{H}$ & 1.189 & 1.704 & 0.832 \\
\hline $\mathrm{H}$ & 0.377 & 0.181 & 1.228 \\
\hline $\mathrm{H}$ & 0.141 & 0.250 & -1.615 \\
\hline $\mathrm{H}$ & -2.249 & 1.041 & -1.196 \\
\hline $\mathrm{H}$ & -1.862 & 1.253 & 0.527 \\
\hline $\mathrm{H}$ & -0.633 & 2.541 & -2.212 \\
\hline $\mathrm{H}$ & -0.292 & 3.132 & -0.554 \\
\hline $\mathrm{H}$ & 1.053 & 2.617 & -1.622 \\
\hline \multicolumn{4}{|c|}{32} \\
\hline \multicolumn{4}{|c|}{ Structure 44, } \\
\hline $\mathrm{C}$ & -3.383 & -1.979 & 0.387 \\
\hline $\mathrm{P}$ & 2.098 & -0.204 & -0.403 \\
\hline $\mathrm{P}$ & -2.212 & -1.000 & 1.346 \\
\hline 0 & 1.496 & -1.537 & -0.689 \\
\hline $\mathrm{C}$ & 3.030 & -0.143 & 1.139 \\
\hline $\mathrm{C}$ & 3.284 & 0.341 & -1.646 \\
\hline 0 & -2.848 & -0.179 & 2.415 \\
\hline $\mathrm{C}$ & -1.035 & -2.221 & 1.958 \\
\hline $\mathrm{H}$ & -3.930 & -2.677 & 1.053 \\
\hline $\mathrm{H}$ & -2.852 & -2.567 & -0.389 \\
\hline $\mathrm{H}$ & -4.118 & -1.313 & -0.110 \\
\hline $\mathrm{H}$ & 3.881 & -0.852 & 1.096 \\
\hline $\mathrm{H}$ & 2.381 & -0.423 & 1.993 \\
\hline $\mathrm{H}$ & 3.426 & 0.879 & 1.312 \\
\hline $\mathrm{H}$ & 4.118 & -0.387 & -1.720 \\
\hline $\mathrm{H}$ & 3.700 & 1.333 & -1.377 \\
\hline $\mathrm{H}$ & 2.790 & 0.417 & -2.636 \\
\hline $\mathrm{H}$ & -1.547 & -2.934 & 2.636 \\
\hline $\mathrm{H}$ & -0.220 & -1.723 & 2.522 \\
\hline $\mathrm{H}$ & -0.591 & -2.788 & 1.115 \\
\hline $\mathrm{C}$ & -1.319 & -0.039 & 0.093 \\
\hline $\mathrm{C}$ & -0.246 & 0.908 & 0.690 \\
\hline $\mathrm{C}$ & 0.901 & 1.160 & -0.315 \\
\hline $\mathrm{C}$ & -0.882 & 2.256 & 1.085 \\
\hline $\mathrm{H}$ & -2.055 & 0.543 & -0.500 \\
\hline $\mathrm{H}$ & -0.860 & -0.762 & -0.610 \\
\hline $\mathrm{H}$ & 0.186 & 0.455 & 1.612 \\
\hline $\mathrm{H}$ & 1.464 & 2.074 & -0.030 \\
\hline $\mathrm{H}$ & 0.489 & 1.343 & -1.329 \\
\hline $\mathrm{H}$ & -0.139 & 2.934 & 1.562 \\
\hline $\mathrm{H}$ & -1.296 & 2.790 & 0.201 \\
\hline $\mathrm{H}$ & -1.712 & 2.128 & 1.813 \\
\hline \multicolumn{4}{|c|}{27} \\
\hline \multicolumn{4}{|c|}{ Structure 45, Gd cor } \\
\hline $\mathrm{C}$ & -1.863 & 1.595 & 0.944 \\
\hline $\mathrm{P}$ & 1.567 & -0.038 & 0.008 \\
\hline $\mathrm{P}$ & -1.472 & -0.001 & 0.206 \\
\hline O & 1.455 & -1.102 & 1.048 \\
\hline $\mathrm{C}$ & 2.090 & 1.546 & 0.687 \\
\hline $\mathrm{C}$ & 2.802 & -0.396 & -1.255 \\
\hline O & -1.253 & -1.068 & 1.224 \\
\hline $\mathrm{C}$ & -2.869 & -0.326 & -0.886 \\
\hline $\mathrm{H}$ & -2.757 & 1.499 & 1.594 \\
\hline $\mathrm{H}$ & -2.079 & 2.350 & 0.161 \\
\hline $\mathrm{H}$ & -1.016 & 1.950 & 1.565 \\
\hline $\mathrm{H}$ & 3.057 & 1.427 & 1.216 \\
\hline $\mathrm{H}$ & 1.339 & 1.921 & 1.412 \\
\hline $\mathrm{H}$ & 2.221 & 2.297 & -0.118 \\
\hline $\mathrm{H}$ & 3.797 & -0.531 & -0.783 \\
\hline $\mathrm{H}$ & 2.867 & 0.434 & -1.987 \\
\hline $\mathrm{H}$ & 2.536 & -1.329 & -1.793 \\
\hline $\mathrm{H}$ & -3.797 & -0.437 & -0.290 \\
\hline $\mathrm{H}$ & -2.697 & -1.264 & -1.453 \\
\hline
\end{tabular}




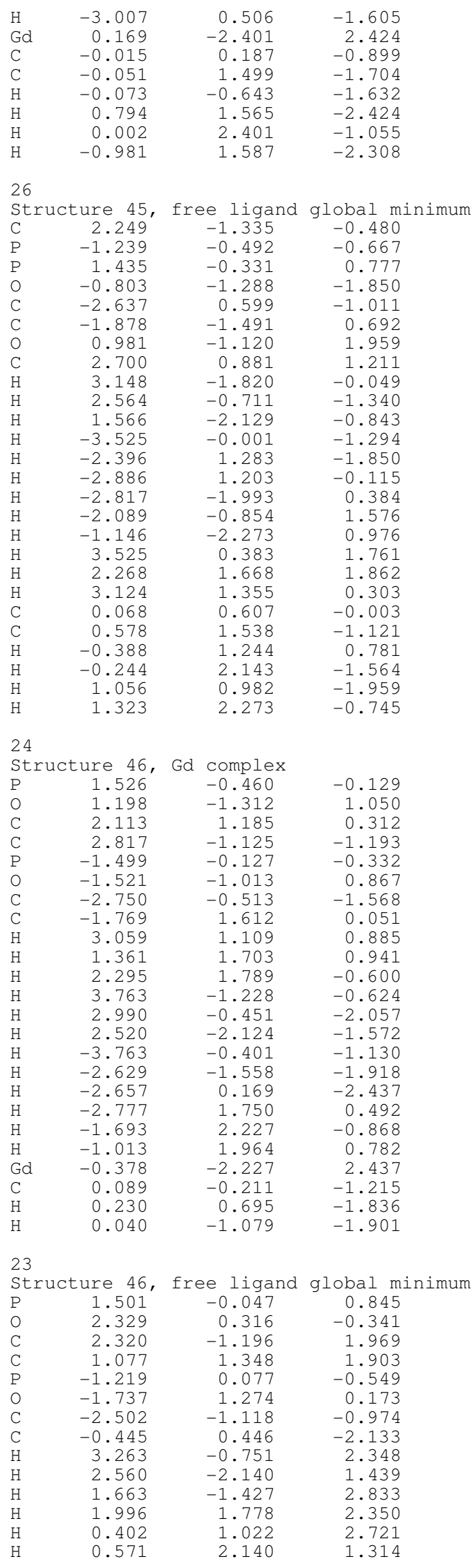




\begin{tabular}{|c|c|c|c|}
\hline $\mathrm{H}$ & -3.263 & -0.643 & -1.627 \\
\hline $\mathrm{H}$ & -3.002 & -1.483 & -0.053 \\
\hline $\mathrm{H}$ & -2.063 & -1.984 & -1.509 \\
\hline $\mathrm{H}$ & -1.196 & 0.865 & -2.833 \\
\hline $\mathrm{H}$ & -0.017 & -0.475 & -2.579 \\
\hline $\mathrm{H}$ & 0.365 & 1.192 & -2.002 \\
\hline $\mathrm{C}$ & -0.040 & -0.898 & 0.421 \\
\hline $\mathrm{H}$ & 0.214 & -1.814 & -0.147 \\
\hline $\mathrm{H}$ & -0.539 & -1.216 & 1.357 \\
\hline \multicolumn{4}{|c|}{30} \\
\hline \multicolumn{4}{|c|}{ Structure 47, Gd complex } \\
\hline $\mathrm{C}$ & 1.496 & 0.657 & -2.102 \\
\hline $\mathrm{P}$ & -1.607 & 0.346 & 0.184 \\
\hline $\mathrm{P}$ & 1.378 & 0.036 & -0.412 \\
\hline O & -1.601 & -1.146 & 0.172 \\
\hline $\mathrm{C}$ & -2.234 & 1.043 & -1.358 \\
\hline $\mathrm{C}$ & -2.754 & 1.046 & 1.387 \\
\hline 0 & 1.073 & -1.423 & -0.361 \\
\hline $\mathrm{C}$ & 3.010 & 0.449 & 0.236 \\
\hline $\mathrm{H}$ & 2.282 & 0.099 & -2.652 \\
\hline $\mathrm{H}$ & 1.761 & 1.733 & -2.111 \\
\hline $\mathrm{H}$ & 0.536 & 0.516 & -2.636 \\
\hline $\mathrm{H}$ & -3.262 & 0.674 & -1.545 \\
\hline $\mathrm{H}$ & -1.596 & 0.737 & -2.210 \\
\hline $\mathrm{H}$ & -2.264 & 2.150 & -1.307 \\
\hline $\mathrm{H}$ & -3.794 & 0.763 & 1.125 \\
\hline $\mathrm{H}$ & -2.682 & 2.152 & 1.401 \\
\hline $\mathrm{H}$ & -2.537 & 0.656 & 2.402 \\
\hline $\mathrm{H}$ & 3.794 & -0.023 & -0.390 \\
\hline $\mathrm{H}$ & 3.122 & 0.070 & 1.272 \\
\hline $\mathrm{H}$ & 3.168 & 1.546 & 0.233 \\
\hline $\mathrm{Gd}$ & -0.444 & -3.108 & -0.053 \\
\hline $\mathrm{C}$ & 0.183 & 2.510 & 0.175 \\
\hline $\mathrm{C}$ & 0.094 & 1.009 & 0.506 \\
\hline $\mathrm{C}$ & 0.375 & 0.810 & 2.015 \\
\hline $\mathrm{H}$ & -0.009 & 2.725 & -0.899 \\
\hline $\mathrm{H}$ & 1.187 & 2.927 & 0.413 \\
\hline $\mathrm{H}$ & -0.550 & 3.108 & 0.760 \\
\hline $\mathrm{H}$ & 0.325 & -0.259 & 2.324 \\
\hline $\mathrm{H}$ & -0.348 & 1.367 & 2.652 \\
\hline $\mathrm{H}$ & 1.379 & 1.188 & 2.307 \\
\hline \multicolumn{4}{|c|}{29} \\
\hline \multicolumn{4}{|c|}{ Structure 47 , } \\
\hline $\mathrm{C}$ & -0.435 & 2.394 & 0.356 \\
\hline $\mathrm{P}$ & -0.673 & -1.104 & 0.836 \\
\hline $\mathrm{P}$ & 0.281 & 1.260 & -0.852 \\
\hline $\mathrm{O}$ & -1.177 & -0.301 & 1.987 \\
\hline $\mathrm{C}$ & -0.181 & -2.771 & 1.327 \\
\hline $\mathrm{C}$ & -1.950 & -1.449 & -0.393 \\
\hline $\mathrm{O}$ & -0.615 & 1.011 & -2.018 \\
\hline $\mathrm{C}$ & 1.791 & 2.134 & -1.322 \\
\hline $\mathrm{H}$ & -0.611 & 3.382 & -0.116 \\
\hline $\mathrm{H}$ & 0.245 & 2.535 & 1.220 \\
\hline $\mathrm{H}$ & -1.408 & 2.009 & 0.721 \\
\hline $\mathrm{H}$ & -1.022 & -3.271 & 1.850 \\
\hline $\mathrm{H}$ & 0.685 & -2.734 & 2.018 \\
\hline $\mathrm{H}$ & 0.089 & $-3 \cdot 382$ & 0.442 \\
\hline $\mathrm{H}$ & -2.763 & -2.045 & 0.069 \\
\hline $\mathrm{H}$ & -1.536 & -2.023 & -1.245 \\
\hline $\mathrm{H}$ & -2.389 & -0.504 & -0.772 \\
\hline $\mathrm{H}$ & 1.530 & 3.071 & -1.856 \\
\hline $\mathrm{H}$ & 2.408 & 1.509 & -1.997 \\
\hline $\mathrm{H}$ & 2.389 & 2.401 & -0.428 \\
\hline $\mathrm{C}$ & 1.838 & -0.016 & 1.078 \\
\hline $\mathrm{C}$ & 0.767 & -0.304 & 0.002 \\
\hline $\mathrm{C}$ & 1.369 & -1.254 & -1.056 \\
\hline $\mathrm{H}$ & 1.483 & 0.689 & 1.863 \\
\hline $\mathrm{H}$ & 2.763 & 0.419 & 0.642 \\
\hline $\mathrm{H}$ & 2.158 & -0.943 & 1.603 \\
\hline $\mathrm{H}$ & 0.643 & -1.531 & -1.852 \\
\hline $\mathrm{H}$ & 1.738 & -2.202 & -0.606 \\
\hline $\mathrm{H}$ & 2.247 & -0.801 & -1.568 \\
\hline \multicolumn{4}{|c|}{30} \\
\hline \multicolumn{4}{|c|}{ Structure 48, Gd complex } \\
\hline $\mathrm{C}$ & 3.339 & -0.016 & 0.294 \\
\hline $\mathrm{P}$ & -1.643 & 0.173 & -0.853 \\
\hline $\mathrm{P}$ & 1.705 & 0.249 & 1.012 \\
\hline 0 & -1.502 & -1.125 & -0.136 \\
\hline
\end{tabular}




\begin{tabular}{|c|c|c|c|}
\hline & & & \\
\hline $\mathrm{C}$ & -1.191 & 0.090 & -2.596 \\
\hline $\mathrm{C}$ & -3.341 & 0.780 & -0.913 \\
\hline 0 & 0.986 & -1.018 & 1.324 \\
\hline C & 2.070 & 1.254 & 2.463 \\
\hline $\mathrm{H}$ & 3.976 & -0.588 & 0.998 \\
\hline $\mathrm{H}$ & 3.829 & 0.955 & 0.077 \\
\hline $\mathrm{H}$ & 3.250 & -0.591 & -0.651 \\
\hline $\mathrm{H}$ & -1.882 & -0.590 & -3.134 \\
\hline $\mathrm{H}$ & -0.160 & -0.302 & -2.709 \\
\hline $\mathrm{H}$ & -1.247 & 1.096 & -3.059 \\
\hline $\mathrm{H}$ & -3.976 & 0.072 & -1.483 \\
\hline $\mathrm{H}$ & -3.380 & 1.772 & -1.408 \\
\hline $\mathrm{H}$ & -3.753 & 0.873 & 0.112 \\
\hline $\mathrm{H}$ & 2.765 & 0.709 & 3.134 \\
\hline $\mathrm{H}$ & 1.144 & 1.474 & 3.030 \\
\hline $\mathrm{H}$ & 2.543 & 2.211 & 2.163 \\
\hline Go & -0.605 & -2.647 & 1.292 \\
\hline C & 0.877 & 1.297 & -0.223 \\
\hline $\mathrm{C}$ & -0.653 & 1.529 & -0.115 \\
\hline $\mathrm{C}$ & -1.117 & 1.828 & 1.322 \\
\hline $\mathrm{H}$ & 1.124 & 0.890 & -1.225 \\
\hline $\mathrm{H}$ & 1.384 & 2.284 & -0.178 \\
\hline $\mathrm{H}$ & -0.872 & 2.439 & -0.716 \\
\hline $\mathrm{H}$ & -2.178 & 2.163 & 1.353 \\
\hline $\mathrm{H}$ & -0.518 & 2.647 & 1.777 \\
\hline $\mathrm{H}$ & -1.040 & 0.940 & 1.989 \\
\hline 29 & & & \\
\hline & cure 48 , & free ligand & global minimum \\
\hline $\mathrm{C}$ & 2.282 & -1.576 & -1.569 \\
\hline $\mathrm{P}$ & -1.530 & 1.182 & 0.585 \\
\hline $\mathrm{P}$ & 1.691 & -1.389 & 0.122 \\
\hline 0 & -1.182 & 2.456 & 1.276 \\
\hline $\mathrm{C}$ & -1.880 & -0.189 & 1.704 \\
\hline $\mathrm{C}$ & -3.032 & 1.277 & -0.410 \\
\hline O & 0.666 & -2.395 & 0.521 \\
\hline $\mathrm{C}$ & 3.188 & -1.433 & 1.124 \\
\hline C & 1.113 & 0.325 & 0.254 \\
\hline C & -0.216 & 0.568 & -0.530 \\
\hline C & 0.030 & 1.566 & -1.678 \\
\hline $\mathrm{H}$ & 2.762 & -2.568 & -1.696 \\
\hline $\mathrm{H}$ & 1.436 & -1.498 & -2.282 \\
\hline $\mathrm{H}$ & 3.025 & -0.787 & -1.809 \\
\hline $\mathrm{H}$ & -2.751 & 0.057 & 2.345 \\
\hline $\mathrm{H}$ & -2.110 & -1.109 & 1.129 \\
\hline $\mathrm{H}$ & -1.007 & -0.387 & 2.359 \\
\hline $\mathrm{H}$ & -3.905 & 1.485 & 0.241 \\
\hline $\mathrm{H}$ & -2.949 & 2.092 & -1.157 \\
\hline $\mathrm{H}$ & -3.204 & 0.319 & -0.942 \\
\hline $\mathrm{H}$ & 3.672 & -2.428 & 1.041 \\
\hline $\mathrm{H}$ & 3.905 & -0.658 & 0.782 \\
\hline $\mathrm{H}$ & 2.940 & -1.245 & 2.188 \\
\hline $\mathrm{H}$ & 1.916 & 0.993 & -0.119 \\
\hline $\mathrm{H}$ & 0.998 & 0.558 & 1.332 \\
\hline $\mathrm{H}$ & -0.567 & -0.384 & -0.981 \\
\hline $\mathrm{H}$ & 0.340 & 2.568 & -1.303 \\
\hline $\mathrm{H}$ & 0.834 & 1.209 & -2.359 \\
\hline $\mathrm{H}$ & -0.876 & 1.712 & -2.308 \\
\hline 36 & & & \\
\hline & Eure 49, & Gd complex & \\
\hline $\mathrm{C}$ & 3.450 & 0.662 & -0.830 \\
\hline $\mathrm{P}$ & -2.099 & -0.550 & 0.114 \\
\hline $\mathrm{P}$ & 2.340 & 0.004 & 0.425 \\
\hline O & -1.265 & -1.758 & -0.155 \\
\hline $\mathrm{C}$ & -3.836 & -0.781 & -0.326 \\
\hline $\mathrm{C}$ & -2.268 & -0.215 & 1.882 \\
\hline 0 & 1.979 & -1.428 & 0.230 \\
\hline $\mathrm{C}$ & 3.152 & 0.348 & 1.995 \\
\hline $\mathrm{H}$ & 4.412 & 0.111 & -0.811 \\
\hline $\mathrm{H}$ & 3.650 & 1.737 & -0.644 \\
\hline $\mathrm{H}$ & 2.997 & 0.551 & -1.836 \\
\hline $\mathrm{H}$ & -4.277 & -1.591 & 0.290 \\
\hline $\mathrm{H}$ & -3.930 & -1.070 & -1.392 \\
\hline $\mathrm{H}$ & -4.412 & 0.150 & -0.149 \\
\hline $\mathrm{H}$ & -2.732 & -1.088 & 2.384 \\
\hline $\mathrm{H}$ & -2.913 & 0.670 & 2.057 \\
\hline $\mathrm{H}$ & -1.281 & -0.040 & 2.351 \\
\hline $\mathrm{H}$ & 4.103 & -0.219 & 2.063 \\
\hline $\mathrm{H}$ & 2.496 & 0.045 & 2.836 \\
\hline $\mathrm{H}$ & 3.374 & 1.431 & 2.086 \\
\hline
\end{tabular}




\begin{tabular}{|c|c|c|c|}
\hline $\mathrm{Gd}$ & 0.549 & -3.141 & -0.214 \\
\hline $\mathrm{C}$ & -2.048 & 0.911 & -2.220 \\
\hline $\mathrm{C}$ & -1.483 & 0.946 & -0.781 \\
\hline $\mathrm{C}$ & 0.065 & 0.916 & -0.899 \\
\hline $\mathrm{C}$ & 0.878 & 1.077 & 0.409 \\
\hline $\mathrm{C}$ & -1.956 & 2.244 & -0.100 \\
\hline $\mathrm{H}$ & -3.155 & 1.016 & -2.244 \\
\hline $\mathrm{H}$ & -1.645 & 1.746 & -2.836 \\
\hline $\mathrm{H}$ & -1.790 & -0.033 & -2.752 \\
\hline $\mathrm{H}$ & 0.385 & 1.725 & -1.595 \\
\hline $\mathrm{H}$ & 0.351 & -0.030 & -1.412 \\
\hline $\mathrm{H}$ & 0.269 & 0.807 & 1.291 \\
\hline $\mathrm{H}$ & 1.193 & 2.132 & 0.548 \\
\hline $\mathrm{H}$ & -1.619 & 3.141 & -0.666 \\
\hline $\mathrm{H}$ & -3.065 & 2.304 & -0.035 \\
\hline $\mathrm{H}$ & -1.558 & 2.354 & 0.932 \\
\hline \multicolumn{4}{|l|}{35} \\
\hline \multicolumn{4}{|c|}{ Structure 49, } \\
\hline $\mathrm{C}$ & 3.660 & 0.408 & -0.670 \\
\hline $\mathrm{P}$ & -2.267 & 0.721 & -0.979 \\
\hline $\mathrm{P}$ & 2.414 & -0.033 & 0.556 \\
\hline 0 & -1.399 & 1.236 & -2.078 \\
\hline $\mathrm{C}$ & -2.461 & 1.909 & 0.366 \\
\hline $\mathrm{C}$ & -3.972 & 0.468 & -1.516 \\
\hline 0 & 2.772 & -1.224 & 1.379 \\
\hline $\mathrm{C}$ & 2.190 & 1.479 & 1.511 \\
\hline $\mathrm{H}$ & 4.622 & 0.641 & -0.168 \\
\hline $\mathrm{H}$ & 3.335 & 1.297 & -1.249 \\
\hline $\mathrm{H}$ & 3.820 & -0.436 & -1.371 \\
\hline $\mathrm{H}$ & -2.892 & 2.853 & -0.026 \\
\hline $\mathrm{H}$ & -1.477 & 2.141 & 0.822 \\
\hline $\mathrm{H}$ & -3.138 & 1.515 & 1.150 \\
\hline $\mathrm{H}$ & -4.366 & 1.403 & -1.965 \\
\hline $\mathrm{H}$ & -4.622 & 0.194 & -0.661 \\
\hline $\mathrm{H}$ & -4.022 & -0.334 & -2.280 \\
\hline $\mathrm{H}$ & 3.137 & 1.749 & 2.022 \\
\hline $\mathrm{H}$ & 1.403 & 1.335 & 2.280 \\
\hline $\mathrm{H}$ & 1.891 & 2.315 & 0.847 \\
\hline $\mathrm{C}$ & -2.668 & -1.403 & 0.778 \\
\hline $\mathrm{C}$ & -1.655 & -0.862 & -0.256 \\
\hline $\mathrm{C}$ & -0.296 & -0.658 & 0.463 \\
\hline $\mathrm{C}$ & 0.897 & -0.235 & -0.415 \\
\hline $\mathrm{C}$ & -1.516 & -1.902 & $-1 \cdot 388$ \\
\hline $\mathrm{H}$ & -3.664 & -1.614 & 0.330 \\
\hline $\mathrm{H}$ & -2.317 & -2.362 & 1. 222 \\
\hline $\mathrm{H}$ & -2.825 & -0.702 & 1.627 \\
\hline $\mathrm{H}$ & -0.029 & -1.614 & 0.968 \\
\hline $\mathrm{H}$ & -0.431 & 0.083 & 1.283 \\
\hline $\mathrm{H}$ & 0.698 & 0.726 & -0.931 \\
\hline $\mathrm{H}$ & 1.094 & -0.997 & -1.196 \\
\hline $\mathrm{H}$ & -1.075 & -2.853 & -1.013 \\
\hline $\mathrm{H}$ & -2.499 & -2.164 & -1.840 \\
\hline $\mathrm{H}$ & -0.864 & -1.548 & -2.218 \\
\hline \multicolumn{4}{|l|}{33} \\
\hline \multicolumn{4}{|c|}{ Str } \\
\hline $\mathrm{C}$ & 3.704 & -0.704 & -0.438 \\
\hline $\mathrm{P}$ & -1.971 & 0.239 & -0.326 \\
\hline $\mathrm{P}$ & 2.216 & -1.124 & 0.485 \\
\hline 0 & -1.599 & -1.091 & -0.887 \\
\hline $\mathrm{C}$ & -3.549 & 0.863 & -0.939 \\
\hline $\mathrm{C}$ & -2.297 & 0.193 & 1. 450 \\
\hline 0 & 1. 388 & -2.189 & -0.148 \\
\hline $\mathrm{C}$ & 2.795 & -1.533 & 2.140 \\
\hline $\mathrm{H}$ & 4.365 & -1.591 & -0.515 \\
\hline $\mathrm{H}$ & 4.257 & 0.112 & 0.070 \\
\hline $\mathrm{H}$ & 3.436 & -0.371 & -1.462 \\
\hline $\mathrm{H}$ & -4.365 & 0.165 & -0.661 \\
\hline $\mathrm{H}$ & -3.528 & 0.947 & -2.045 \\
\hline $\mathrm{H}$ & -3.770 & 1.859 & -0.503 \\
\hline $\mathrm{H}$ & -3.202 & -0.416 & 1.652 \\
\hline $\mathrm{H}$ & -2.465 & 1.217 & 1.841 \\
\hline $\mathrm{H}$ & -1.449 & -0.266 & 1.994 \\
\hline $\mathrm{H}$ & 3.432 & -2.440 & 2.107 \\
\hline $\mathrm{H}$ & 1.930 & -1.730 & 2.807 \\
\hline $\mathrm{H}$ & 3.387 & -0.695 & 2.561 \\
\hline Gd & -0.441 & -3.029 & -1.209 \\
\hline $\mathrm{C}$ & 1.301 & 0.429 & 0.680 \\
\hline $\mathrm{C}$ & 0.731 & 0.935 & -0.668 \\
\hline $\mathrm{C}$ & -0.697 & 1.533 & -0.609 \\
\hline
\end{tabular}




\begin{tabular}{|c|c|c|c|}
\hline $\mathrm{C}$ & -0.969 & 2.267 & -1.939 \\
\hline $\mathrm{H}$ & 1.966 & 1.193 & 1.133 \\
\hline $\mathrm{H}$ & 0.489 & 0.247 & 1.408 \\
\hline $\mathrm{H}$ & 1.431 & 1.709 & -1.060 \\
\hline $\mathrm{H}$ & 0.741 & 0.124 & -1.431 \\
\hline $\mathrm{H}$ & -0.740 & 2.281 & 0.212 \\
\hline $\mathrm{H}$ & -1.937 & 2.814 & -1.927 \\
\hline $\mathrm{H}$ & -0.186 & 3.029 & -2.149 \\
\hline $\mathrm{H}$ & -0.984 & 1.569 & -2.807 \\
\hline \multicolumn{4}{|l|}{32} \\
\hline \multicolumn{2}{|c|}{ Structure 50, } & free ligand & global minimum \\
\hline $\mathrm{C}$ & 3.475 & -0.992 & -1.029 \\
\hline $\mathrm{P}$ & -2.000 & 1.403 & -0.515 \\
\hline $\mathrm{P}$ & 2.094 & -1.512 & 0.005 \\
\hline 0 & -0.842 & 2.082 & -1.164 \\
\hline $\mathrm{C}$ & -2.193 & 1.849 & 1.222 \\
\hline $\mathrm{C}$ & -3.577 & 1.886 & -1.248 \\
\hline 0 & 1.943 & -2.991 & 0.112 \\
\hline $\mathrm{C}$ & 2.394 & -0.690 & 1.581 \\
\hline $\mathrm{H}$ & 4.427 & -1.385 & -0.617 \\
\hline $\mathrm{H}$ & 3.534 & 0.115 & -1.069 \\
\hline $\mathrm{H}$ & 3.346 & $-1 \cdot 380$ & -2.060 \\
\hline $\mathrm{H}$ & -2.296 & 2.949 & 1.318 \\
\hline $\mathrm{H}$ & -1.304 & 1.529 & 1.802 \\
\hline $\mathrm{H}$ & -3.095 & 1.373 & 1.655 \\
\hline $\mathrm{H}$ & -3.661 & 2.991 & -1.264 \\
\hline $\mathrm{H}$ & -4.427 & 1.479 & -0.665 \\
\hline $\mathrm{H}$ & -3.643 & 1.511 & -2.291 \\
\hline $\mathrm{H}$ & 3.344 & -1.052 & 2.023 \\
\hline $\mathrm{H}$ & 1.570 & -0.907 & 2.291 \\
\hline $\mathrm{H}$ & 2.463 & 0.408 & 1.438 \\
\hline $\mathrm{C}$ & 0.661 & -0.673 & -0.722 \\
\hline $\mathrm{C}$ & -0.642 & -0.999 & 0.028 \\
\hline $\mathrm{C}$ & -1.922 & -0.425 & -0.627 \\
\hline $\mathrm{C}$ & -3.158 & -1.090 & 0.011 \\
\hline $\mathrm{H}$ & 0.579 & -0.988 & -1.783 \\
\hline $\mathrm{H}$ & 0.867 & 0.415 & -0.714 \\
\hline $\mathrm{H}$ & -0.748 & -2.108 & 0.078 \\
\hline $\mathrm{H}$ & -0.574 & -0.651 & 1.084 \\
\hline $\mathrm{H}$ & -1.910 & -0.705 & -1.703 \\
\hline $\mathrm{H}$ & -4.109 & -0.759 & -0.461 \\
\hline $\mathrm{H}$ & -3.124 & -2.197 & -0.104 \\
\hline $\mathrm{H}$ & -3.233 & -0.885 & 1.102 \\
\hline 36 & & & \\
\hline Str & ture 51, & Gd complex & \\
\hline $\mathrm{C}$ & -3.233 & -0.410 & 1.643 \\
\hline $\mathrm{P}$ & 1.871 & 0.086 & -0.093 \\
\hline $\mathrm{P}$ & -2.145 & -1.033 & 0.353 \\
\hline 0 & 1.506 & -1.354 & -0.194 \\
\hline $\mathrm{C}$ & 3.210 & 0.364 & 1.090 \\
\hline $\mathrm{C}$ & 2.569 & 0.772 & -1.602 \\
\hline 0 & -1.080 & -1.949 & 0.849 \\
\hline $\mathrm{C}$ & -3.315 & -1.882 & -0.733 \\
\hline $\mathrm{H}$ & -3.827 & -1.243 & 2.070 \\
\hline $\mathrm{H}$ & -3.932 & 0.347 & 1.231 \\
\hline $\mathrm{H}$ & -2.641 & 0.043 & 2.463 \\
\hline $\mathrm{H}$ & 4.108 & -0.214 & 0.788 \\
\hline $\mathrm{H}$ & 2.900 & 0.030 & 2.101 \\
\hline $\mathrm{H}$ & 3.476 & 1.440 & 1.133 \\
\hline $\mathrm{H}$ & 3.551 & 0.300 & -1.810 \\
\hline $\mathrm{H}$ & 2.717 & 1.867 & -1.500 \\
\hline $\mathrm{H}$ & 1.903 & 0.569 & -2.463 \\
\hline $\mathrm{H}$ & -3.790 & -2.726 & -0.194 \\
\hline $\mathrm{H}$ & -2.786 & -2.284 & -1.621 \\
\hline $\mathrm{H}$ & -4.108 & -1.185 & -1.073 \\
\hline $\mathrm{Gd}$ & 0.711 & -3.327 & 0.608 \\
\hline $\mathrm{C}$ & -1.522 & 0.270 & -0.747 \\
\hline $\mathrm{C}$ & -0.716 & 1.473 & -0.196 \\
\hline $\mathrm{C}$ & 0.554 & 1.116 & 0.617 \\
\hline $\mathrm{C}$ & -0.344 & 2.371 & -1.401 \\
\hline $\mathrm{C}$ & -1.615 & 2.348 & 0.714 \\
\hline $\mathrm{H}$ & -2.403 & 0.667 & -1.292 \\
\hline $\mathrm{H}$ & -0.909 & -0.245 & -1.515 \\
\hline $\mathrm{H}$ & 0.243 & 0.612 & 1.555 \\
\hline $\mathrm{H}$ & 1.036 & 2.063 & 0.937 \\
\hline $\mathrm{H}$ & -1.248 & 2.823 & -1.868 \\
\hline $\mathrm{H}$ & 0.171 & 1.807 & -2.206 \\
\hline $\mathrm{H}$ & 0.322 & 3.213 & -1.106 \\
\hline $\mathrm{H}$ & -2.597 & 2.570 & 0.240 \\
\hline
\end{tabular}




\begin{tabular}{|c|c|c|c|}
\hline $\begin{array}{l}\mathrm{H} \\
\mathrm{H}\end{array}$ & $\begin{array}{l}-1.819 \\
-1.137\end{array}$ & $\begin{array}{l}1.868 \\
3.327\end{array}$ & $\begin{array}{l}1.693 \\
0.941\end{array}$ \\
\hline \multicolumn{4}{|l|}{35} \\
\hline Str & ture 51, & free ligand & global minimum \\
\hline $\mathrm{C}$ & -2.413 & -1.333 & 1.028 \\
\hline $\mathrm{P}$ & -0.762 & 2.004 & 0.104 \\
\hline $\mathrm{P}$ & -0.954 & -2.244 & 0.488 \\
\hline O & -1.864 & 1.414 & -0.707 \\
\hline C & 0.084 & 3.382 & -0.696 \\
\hline $\mathrm{C}$ & -1.326 & 2.755 & 1.647 \\
\hline O & -0.098 & -2.726 & 1.608 \\
\hline $\mathrm{C}$ & -1.693 & -3.583 & -0.474 \\
\hline $\mathrm{H}$ & -3.056 & -1.987 & 1.652 \\
\hline $\mathrm{H}$ & -3.002 & -0.989 & 0.155 \\
\hline $\mathrm{H}$ & -2.121 & -0.455 & 1.637 \\
\hline $\mathrm{H}$ & -0.626 & 4.220 & -0.853 \\
\hline $\mathrm{H}$ & 0.484 & 3.079 & -1.683 \\
\hline $\mathrm{H}$ & 0.922 & 3.741 & -0.065 \\
\hline $\mathrm{H}$ & -2.046 & 3.571 & 1.432 \\
\hline $\mathrm{H}$ & -0.467 & 3.176 & 2.209 \\
\hline $\mathrm{H}$ & -1.829 & 1.997 & 2.281 \\
\hline $\mathrm{H}$ & -2.331 & -4.213 & 0.178 \\
\hline $\mathrm{H}$ & -0.899 & -4.220 & -0.913 \\
\hline $\mathrm{H}$ & -2.316 & -3.172 & -1.295 \\
\hline $\mathrm{C}$ & -0.085 & -1.277 & -0.781 \\
\hline C & 0.993 & -0.250 & -0.344 \\
\hline $\mathrm{C}$ & 0.493 & 0.816 & 0.662 \\
\hline C & 2.189 & -0.987 & 0.309 \\
\hline $\mathrm{C}$ & 1.544 & 0.422 & -1.624 \\
\hline $\mathrm{H}$ & -0.858 & -0.777 & -1.399 \\
\hline $\mathrm{H}$ & 0.396 & -2.013 & -1.458 \\
\hline $\mathrm{H}$ & 1.357 & 1.413 & 1.021 \\
\hline $\mathrm{H}$ & 0.093 & 0.308 & 1.562 \\
\hline $\mathrm{H}$ & 2.551 & -1.832 & -0.319 \\
\hline $\mathrm{H}$ & 1.938 & -1.403 & 1.308 \\
\hline $\mathrm{H}$ & 3.056 & -0.305 & 0.462 \\
\hline $\mathrm{H}$ & 2.063 & -0.312 & -2.281 \\
\hline $\mathrm{H}$ & 0.739 & 0.878 & -2.239 \\
\hline $\mathrm{H}$ & 2.282 & 1.222 & -1.388 \\
\hline \multicolumn{4}{|l|}{37} \\
\hline Str & cure 52, & Gd complex & \\
\hline $\mathrm{P}$ & 2.350 & -1.567 & -0.653 \\
\hline O & 1.363 & -2.303 & -1.491 \\
\hline $\mathrm{C}$ & 3.356 & -2.663 & 0.370 \\
\hline $\mathrm{C}$ & 3.584 & -0.678 & -1.617 \\
\hline $\mathrm{P}$ & -2.346 & -1.567 & 0.285 \\
\hline O & -1.477 & -2.528 & -0.450 \\
\hline $\mathrm{C}$ & -3.581 & -0.782 & -0.764 \\
\hline $\mathrm{C}$ & $-3 \cdot 324$ & -2.348 & 1.584 \\
\hline $\mathrm{H}$ & 3.915 & -3.374 & -0.272 \\
\hline $\mathrm{H}$ & 2.706 & -3.243 & 1.058 \\
\hline $\mathrm{H}$ & 4.081 & -2.077 & 0.971 \\
\hline $\mathrm{H}$ & 4.201 & -1.398 & -2.192 \\
\hline $\mathrm{H}$ & 4.250 & -0.092 & -0.951 \\
\hline $\mathrm{H}$ & 3.091 & 0.006 & -2.336 \\
\hline $\mathrm{H}$ & -4.250 & -1.552 & -1.199 \\
\hline $\mathrm{H}$ & -3.087 & -0.238 & -1.594 \\
\hline $\mathrm{H}$ & -4.194 & -0.070 & -0.176 \\
\hline $\mathrm{H}$ & -3.970 & -3.138 & 1.149 \\
\hline $\mathrm{H}$ & -3.966 & -1.599 & 2.089 \\
\hline $\mathrm{H}$ & -2.654 & -2.812 & 2.336 \\
\hline $\mathrm{Gd}$ & -0.382 & -3.602 & -2.123 \\
\hline $\mathrm{C}$ & 1.317 & 1.662 & -0.704 \\
\hline $\mathrm{C}$ & 0.576 & 2.714 & -1.240 \\
\hline C & -0.797 & 2.765 & -1.030 \\
\hline $\mathrm{C}$ & -1.416 & 1.771 & -0.273 \\
\hline $\mathrm{C}$ & -0.689 & 0.690 & 0.257 \\
\hline $\mathrm{C}$ & 0.707 & 0.622 & 0.018 \\
\hline $\mathrm{C}$ & -1.449 & -0.259 & 1.160 \\
\hline $\mathrm{C}$ & 1.610 & -0.456 & 0.579 \\
\hline $\mathrm{H}$ & 2.410 & 1.669 & -0.846 \\
\hline $\mathrm{H}$ & 1.078 & 3.511 & -1.813 \\
\hline $\mathrm{H}$ & -1.389 & 3.602 & -1.438 \\
\hline $\mathrm{H}$ & -2.497 & 1.860 & -0.076 \\
\hline $\mathrm{H}$ & -0.784 & -0.731 & 1.908 \\
\hline $\mathrm{H}$ & -2.189 & 0.317 & 1.754 \\
\hline $\mathrm{H}$ & 2.433 & 0.020 & 1.151 \\
\hline $\mathrm{H}$ & 1.071 & -1.095 & 1.303 \\
\hline
\end{tabular}




\begin{tabular}{|c|c|c|c|}
\hline \multicolumn{4}{|c|}{36} \\
\hline & ture 52 , & free ligand & global minimum \\
\hline $\mathrm{P}$ & -2.557 & -1.282 & 0.343 \\
\hline 0 & -2.035 & -1.659 & 1.688 \\
\hline $\mathrm{C}$ & -3.451 & -2.615 & -0.479 \\
\hline $\mathrm{C}$ & -3.737 & 0.079 & 0.375 \\
\hline $\mathrm{P}$ & 2.427 & -1.662 & -0.291 \\
\hline 0 & 1.850 & -2.052 & -1.610 \\
\hline $\mathrm{C}$ & 3.126 & -3.050 & 0.625 \\
\hline $\mathrm{C}$ & 3.789 & -0.489 & -0.411 \\
\hline $\mathrm{H}$ & -4.315 & -2.931 & 0.140 \\
\hline $\mathrm{H}$ & -2.781 & -3.487 & -0.627 \\
\hline $\mathrm{H}$ & -3.825 & -2.280 & -1.468 \\
\hline $\mathrm{H}$ & -4.623 & -0.203 & 0.979 \\
\hline $\mathrm{H}$ & -4.070 & 0.327 & -0.653 \\
\hline $\mathrm{H}$ & -3.273 & 0.978 & 0.830 \\
\hline $\mathrm{H}$ & 3.932 & -3.528 & 0.033 \\
\hline $\mathrm{H}$ & 2.339 & -3.805 & 0.829 \\
\hline $\mathrm{H}$ & 3.548 & -2.704 & 1.590 \\
\hline $\mathrm{H}$ & 4.623 & -0.936 & -0.989 \\
\hline $\mathrm{H}$ & 4.160 & -0.221 & 0.599 \\
\hline $\mathrm{H}$ & 3.456 & 0.433 & -0.928 \\
\hline $\mathrm{C}$ & -0.965 & 1.653 & -0.898 \\
\hline $\mathrm{C}$ & -0.317 & 2.833 & -0.535 \\
\hline $\mathrm{C}$ & 0.802 & 2.781 & 0.291 \\
\hline $\mathrm{C}$ & 1.277 & 1.548 & 0.738 \\
\hline $\mathrm{C}$ & 0.647 & 0.349 & 0.366 \\
\hline $\mathrm{C}$ & -0.511 & 0.405 & -0.442 \\
\hline $\mathrm{C}$ & 1. 232 & -0.950 & 0.872 \\
\hline $\mathrm{C}$ & -1.278 & -0.830 & -0.861 \\
\hline $\mathrm{H}$ & -1.851 & 1.712 & -1.553 \\
\hline $\mathrm{H}$ & -0.691 & 3.805 & -0.898 \\
\hline $\mathrm{H}$ & 1.314 & 3.712 & 0.589 \\
\hline $\mathrm{H}$ & 2.165 & 1.525 & 1.392 \\
\hline $\mathrm{H}$ & 1.743 & -0.787 & 1.844 \\
\hline $\mathrm{H}$ & 0.443 & -1.702 & 1.067 \\
\hline $\mathrm{H}$ & -1.766 & -0.662 & -1.844 \\
\hline $\mathrm{H}$ & -0.605 & -1.698 & -1.000 \\
\hline \multicolumn{4}{|c|}{25} \\
\hline \multicolumn{4}{|c|}{ Str } \\
\hline $\mathrm{C}$ & 2.520 & 1.372 & -1.167 \\
\hline $\mathrm{P}$ & -1.579 & 0.219 & -0.152 \\
\hline $\mathrm{P}$ & 1.579 & 0.219 & -0.154 \\
\hline 0 & -1.344 & -1.089 & -0.826 \\
\hline $\mathrm{C}$ & -2.522 & 1.372 & -1.163 \\
\hline $\mathrm{C}$ & -2.519 & 0.065 & 1.376 \\
\hline 0 & 1.343 & -1.089 & -0.828 \\
\hline $\mathrm{C}$ & 2.522 & 0.064 & 1.371 \\
\hline $\mathrm{H}$ & 3.500 & 0.928 & -1.436 \\
\hline $\mathrm{H}$ & 2.697 & 2.320 & -0.620 \\
\hline $\mathrm{H}$ & 1.964 & 1.597 & -2.100 \\
\hline $\mathrm{H}$ & -3.502 & 0.928 & -1.430 \\
\hline $\mathrm{H}$ & -1.967 & 1.597 & -2.097 \\
\hline $\mathrm{H}$ & -2.698 & 2.320 & -0.615 \\
\hline $\mathrm{H}$ & -3.500 & -0.411 & 1.170 \\
\hline $\mathrm{H}$ & -2.696 & 1.061 & 1.829 \\
\hline $\mathrm{H}$ & -1.963 & -0.565 & 2.100 \\
\hline $\mathrm{H}$ & 3.502 & -0.412 & 1.164 \\
\hline $\mathrm{H}$ & 1.967 & -0.565 & 2.097 \\
\hline $\mathrm{H}$ & 2.700 & 1.061 & 1.825 \\
\hline $\mathrm{Gd}$ & -0.001 & -2.731 & -1.673 \\
\hline $\mathrm{C}$ & 0.000 & 1.027 & 0.263 \\
\hline $\mathrm{C}$ & 0.001 & 2.216 & 0.876 \\
\hline $\mathrm{H}$ & 0.938 & 2.731 & 1.140 \\
\hline $\mathrm{H}$ & -0.936 & 2.731 & 1.142 \\
\hline \multicolumn{4}{|c|}{24} \\
\hline & ture 53, & free ligand & global minimum \\
\hline $\mathrm{C}$ & $1.986^{\prime}$ & 0.378 & 1.318 \\
\hline $\mathrm{P}$ & $-1 \cdot 328$ & -0.721 & 0.239 \\
\hline $\mathrm{P}$ & 1.578 & 0.334 & -0.437 \\
\hline 0 & -0.561 & -1.736 & 1.016 \\
\hline $\mathrm{C}$ & -2.378 & -1.452 & -1.029 \\
\hline $\mathrm{C}$ & -2.459 & 0.238 & 1.261 \\
\hline 0 & 2.254 & 1.415 & $-1 \cdot 211$ \\
\hline $\mathrm{C}$ & 2.067 & $-1 \cdot 314$ & -0.975 \\
\hline $\mathrm{H}$ & 3.086 & 0.331 & 1.451 \\
\hline $\mathrm{H}$ & 1.530 & -0.477 & 1.855 \\
\hline $\mathrm{H}$ & 1.615 & 1.321 & 1.769 \\
\hline $\mathrm{H}$ & -3.087 & -2.170 & -0.567 \\
\hline
\end{tabular}




\begin{tabular}{|c|c|c|c|}
\hline $\mathrm{H}$ & -1.756 & -1.994 & -1.770 \\
\hline $\mathrm{H}$ & -2.960 & -0.669 & -1.555 \\
\hline $\mathrm{H}$ & -3.170 & -0.439 & 1.779 \\
\hline $\mathrm{H}$ & -3.038 & 0.953 & 0.642 \\
\hline $\mathrm{H}$ & -1.890 & 0.807 & 2.025 \\
\hline $\mathrm{H}$ & 3.170 & -1.418 & -0.919 \\
\hline $\mathrm{H}$ & 1.749 & -1.479 & -2.025 \\
\hline $\mathrm{H}$ & 1.608 & -2.095 & -0.337 \\
\hline $\mathrm{C}$ & -0.228 & 0.458 & -0.592 \\
\hline $\mathrm{C}$ & -0.749 & 1.440 & -1.335 \\
\hline $\mathrm{H}$ & -0.105 & 2.170 & -1.851 \\
\hline $\mathrm{H}$ & -1.836 & 1.564 & -1.465 \\
\hline \multicolumn{4}{|c|}{29} \\
\hline \multicolumn{4}{|c|}{ Structure 54, Gd complex } \\
\hline $\mathrm{P}$ & 1.552 & -0.240 & -0.025 \\
\hline $\mathrm{C}$ & 2.249 & 0.994 & -1.144 \\
\hline $\mathrm{C}$ & 1.638 & 2.325 & -0.681 \\
\hline $\mathrm{C}$ & 0.167 & 2.079 & -0.292 \\
\hline $\mathrm{C}$ & 0.088 & 0.737 & 0.490 \\
\hline $\mathrm{C}$ & -0.763 & 1.955 & -1.517 \\
\hline $\mathrm{P}$ & -1.531 & -0.010 & 0.066 \\
\hline $\mathrm{C}$ & -2.072 & 1.315 & -1.037 \\
\hline 0 & 1.244 & -1.557 & -0.650 \\
\hline $\mathrm{C}$ & 2.632 & -0.317 & 1.411 \\
\hline $\mathrm{C}$ & -2.564 & 0.061 & 1.534 \\
\hline $\mathrm{O}$ & -1.437 & -1.353 & -0.573 \\
\hline $\mathrm{Gd}$ & -0.243 & -3.102 & -1.433 \\
\hline $\mathrm{H}$ & 1.946 & 0.754 & -2.185 \\
\hline $\mathrm{H}$ & 3.358 & 0.994 & -1.096 \\
\hline $\mathrm{H}$ & 2.197 & 2.706 & 0.205 \\
\hline $\mathrm{H}$ & 1.720 & 3.102 & -1.475 \\
\hline $\mathrm{H}$ & -0.201 & 2.914 & 0.352 \\
\hline $\mathrm{H}$ & 0.143 & 0.936 & 1.579 \\
\hline $\mathrm{H}$ & -0.298 & 1.314 & -2.301 \\
\hline $\mathrm{H}$ & -0.952 & 2.952 & -1.979 \\
\hline $\mathrm{H}$ & -2.682 & 2.047 & -0.467 \\
\hline $\mathrm{H}$ & -2.684 & 0.914 & -1.872 \\
\hline $\mathrm{H}$ & 2.167 & -0.940 & 2.202 \\
\hline $\mathrm{H}$ & 3.605 & -0.767 & 1.129 \\
\hline $\mathrm{H}$ & 2.813 & 0.700 & 1.816 \\
\hline $\mathrm{H}$ & -2.180 & -0.642 & 2.301 \\
\hline $\mathrm{H}$ & -2.565 & 1.086 & 1.957 \\
\hline $\mathrm{H}$ & -3.605 & -0.224 & 1.279 \\
\hline \multicolumn{4}{|c|}{28} \\
\hline \multicolumn{4}{|c|}{ Structure 54, free ligand global m } \\
\hline $\mathrm{C}$ & 0.366 & 0.971 & -0.970 \\
\hline $\mathrm{C}$ & 1.756 & 0.478 & -1.453 \\
\hline $\mathrm{C}$ & 2.056 & -0.888 & -0.812 \\
\hline $\mathrm{P}$ & 1.463 & -0.709 & 0.884 \\
\hline $\mathrm{C}$ & 0.020 & 0.287 & 0.400 \\
\hline $\mathrm{P}$ & -1.515 & -0.664 & 0.127 \\
\hline $\mathrm{C}$ & -2.055 & 0.430 & -1.201 \\
\hline $\mathrm{C}$ & -0.754 & 0.636 & -1.987 \\
\hline 0 & 1.194 & -1.968 & 1.633 \\
\hline $\mathrm{C}$ & 2.592 & 0.414 & 1.726 \\
\hline 0 & -1.334 & -2.078 & -0.311 \\
\hline $\mathrm{C}$ & -2.583 & -0.442 & 1.552 \\
\hline $\mathrm{H}$ & 0.396 & 2.078 & -0.828 \\
\hline $\mathrm{H}$ & 2.538 & 1.213 & -1.152 \\
\hline $\mathrm{H}$ & 1.800 & 0.415 & -2.564 \\
\hline $\mathrm{H}$ & 3.136 & -1.142 & -0.833 \\
\hline $\mathrm{H}$ & 1.489 & -1.698 & -1.316 \\
\hline $\mathrm{H}$ & -0.168 & 1.069 & 1.163 \\
\hline $\mathrm{H}$ & -2.427 & 1.387 & -0.778 \\
\hline $\mathrm{H}$ & -2.858 & -0.039 & -1.807 \\
\hline $\mathrm{H}$ & -0.866 & 1.451 & -2.739 \\
\hline $\mathrm{H}$ & -0.504 & -0.293 & -2.550 \\
\hline $\mathrm{H}$ & 2.702 & 1.363 & 1.165 \\
\hline $\mathrm{H}$ & 3.590 & -0.059 & 1.828 \\
\hline $\mathrm{H}$ & 2.205 & 0.647 & 2.739 \\
\hline $\mathrm{H}$ & -2.682 & 0.635 & 1.797 \\
\hline $\mathrm{H}$ & -3.590 & -0.854 & 1.339 \\
\hline $\mathrm{H}$ & -2.158 & -0.971 & 2.430 \\
\hline \multicolumn{4}{|c|}{29} \\
\hline \multicolumn{4}{|c|}{ Str } \\
\hline $\mathrm{C}$ & 1.048 & 1.988 & -1.836 \\
\hline $\mathrm{C}$ & 2.221 & 1.170 & -1.226 \\
\hline $\mathrm{P}$ & 1.557 & 0.306 & 0.242 \\
\hline $\mathrm{C}$ & -0.068 & 1.101 & 0.117 \\
\hline
\end{tabular}




\begin{tabular}{|c|c|c|c|}
\hline $\mathrm{C}$ & -0.229 & 1.268 & -1.399 \\
\hline $\mathrm{C}$ & -1.622 & 1.875 & -1.565 \\
\hline $\mathrm{C}$ & -2.576 & 0.967 & -0.739 \\
\hline P & -1.562 & 0.174 & 0.558 \\
\hline 0 & 1.457 & -1.177 & 0.142 \\
\hline C & 2.356 & 0.927 & 1.723 \\
\hline 0 & -1.360 & -1.297 & 0.428 \\
\hline C & -2.097 & 0.739 & 2.175 \\
\hline $\mathrm{H}$ & 1.125 & 2.046 & -2.946 \\
\hline $\mathrm{H}$ & 1.055 & 3.030 & -1.441 \\
\hline $\mathrm{H}$ & 2.590 & 0.410 & -1.946 \\
\hline $\mathrm{H}$ & 3.073 & 1.823 & -0.943 \\
\hline $\mathrm{H}$ & -0.059 & 2.094 & 0.610 \\
\hline $\mathrm{H}$ & -0.237 & 0.269 & -1.903 \\
\hline $\mathrm{H}$ & -1.925 & 1.917 & -2.636 \\
\hline $\mathrm{H}$ & -1.637 & 2.916 & -1.168 \\
\hline $\mathrm{H}$ & -3.406 & 1.549 & -0.285 \\
\hline $\mathrm{H}$ & -3.018 & 0.172 & -1.377 \\
\hline $\mathrm{H}$ & 2.353 & 2.036 & 1.727 \\
\hline $\mathrm{H}$ & 3.406 & 0.571 & 1.765 \\
\hline $\mathrm{H}$ & 1.819 & 0.564 & 2.623 \\
\hline $\mathrm{H}$ & -2.186 & 1.844 & 2.188 \\
\hline $\mathrm{H}$ & -3.083 & 0.297 & 2.424 \\
\hline $\mathrm{H}$ & -1.361 & 0.430 & 2.946 \\
\hline Go & 0.107 & -3.030 & 0.114 \\
\hline \multicolumn{4}{|c|}{28} \\
\hline \multicolumn{4}{|c|}{ Structure 55, } \\
\hline $\mathrm{C}$ & 0.623 & 1.029 & -2.028 \\
\hline $\mathrm{C}$ & 1.890 & 0.262 & -1.564 \\
\hline $\mathrm{P}$ & 1.476 & -0.543 & 0.026 \\
\hline $\mathrm{C}$ & -0.174 & 0.199 & 0.103 \\
\hline $\mathrm{C}$ & -0.568 & 0.322 & -1.374 \\
\hline $\mathrm{C}$ & -1.949 & 0.982 & -1.347 \\
\hline $\mathrm{C}$ & -2.793 & 0.176 & -0.323 \\
\hline $\mathrm{P}$ & -1.620 & -0.600 & 0.846 \\
\hline O & 1.541 & -2.032 & 0.005 \\
\hline $\mathrm{C}$ & 2.448 & 0.238 & 1.321 \\
\hline 0 & -1.635 & -2.090 & 0.847 \\
\hline $\mathrm{C}$ & -1.849 & 0.159 & 2.459 \\
\hline $\mathrm{H}$ & 0.534 & 1.034 & -3.139 \\
\hline $\mathrm{H}$ & 0.671 & 2.090 & -1.691 \\
\hline $\mathrm{H}$ & 2.164 & -0.526 & -2.297 \\
\hline $\mathrm{H}$ & 2.761 & 0.940 & -1.445 \\
\hline $\mathrm{H}$ & -0.081 & 1.221 & 0.525 \\
\hline $\mathrm{H}$ & -0.671 & -0.692 & -1.835 \\
\hline $\mathrm{H}$ & -2.422 & 0.980 & -2.356 \\
\hline $\mathrm{H}$ & -1.860 & 2.044 & -1.020 \\
\hline $\mathrm{H}$ & -3.514 & 0.825 & 0.218 \\
\hline $\mathrm{H}$ & -3.367 & -0.627 & -0.832 \\
\hline $\mathrm{H}$ & 2.371 & 1.342 & 1.250 \\
\hline $\mathrm{H}$ & 3.514 & -0.052 & 1.223 \\
\hline $\mathrm{H}$ & 2.080 & -0.083 & 2.316 \\
\hline $\mathrm{H}$ & -1.854 & 1.265 & 2.370 \\
\hline $\mathrm{H}$ & -2.812 & -0.168 & 2.899 \\
\hline $\mathrm{H}$ & -1.025 & -0.140 & 3.139 \\
\hline \multicolumn{4}{|c|}{35} \\
\hline & cure & Gd complex & \\
\hline $\mathrm{C}$ & 1.743 & 1.535 & -1.650 \\
\hline $\mathrm{C}$ & 2.821 & 0.982 & -0.706 \\
\hline $\mathrm{C}$ & 2.555 & 1.349 & 0.755 \\
\hline $\mathrm{P}$ & 0.951 & 0.704 & 1.274 \\
\hline $\mathrm{C}$ & -0.214 & 1.318 & -0.002 \\
\hline $\mathrm{C}$ & 0.342 & 0.932 & -1.403 \\
\hline $\mathrm{C}$ & -0.601 & 1.389 & -2.540 \\
\hline $\mathrm{C}$ & -1.965 & 0.683 & -2.524 \\
\hline $\mathrm{C}$ & -2.773 & 1.016 & -1.268 \\
\hline $\mathrm{P}$ & -1.857 & 0.529 & 0.208 \\
\hline $\mathrm{C}$ & 0.647 & 1.584 & 2.816 \\
\hline O & 0.873 & -0.779 & 1.403 \\
\hline $\mathrm{C}$ & -2.746 & 1.372 & 1.527 \\
\hline 0 & -1.723 & -0.941 & 0.417 \\
\hline $\mathrm{H}$ & 2.055 & 1.322 & -2.698 \\
\hline $\mathrm{H}$ & 1.697 & 2.645 & -1.557 \\
\hline $\mathrm{H}$ & 2.891 & -0.125 & -0.819 \\
\hline $\mathrm{H}$ & 3.815 & 1.389 & -1.007 \\
\hline $\mathrm{H}$ & 2.558 & 2.452 & 0.881 \\
\hline $\mathrm{H}$ & 3.343 & 0.935 & 1. 418 \\
\hline $\mathrm{H}$ & -0.308 & 2.420 & 0.065 \\
\hline $\mathrm{H}$ & 0.436 & -0.179 & -1.468 \\
\hline
\end{tabular}




\begin{tabular}{|c|c|c|c|}
\hline $\mathrm{H}$ & -0.748 & 2.492 & -2.485 \\
\hline $\mathrm{H}$ & -0.115 & 1.186 & -3.522 \\
\hline $\mathrm{H}$ & -1.821 & -0.419 & -2.608 \\
\hline $\mathrm{H}$ & -2.548 & 0.992 & -3.423 \\
\hline $\mathrm{H}$ & -2.979 & 2.106 & -1.222 \\
\hline $\mathrm{H}$ & -3.752 & 0.492 & -1.276 \\
\hline $\mathrm{H}$ & 0.559 & 2.673 & 2.630 \\
\hline $\mathrm{H}$ & -0.287 & 1.222 & 3.289 \\
\hline $\mathrm{H}$ & 1.484 & 1.408 & 3.522 \\
\hline $\mathrm{H}$ & -2.677 & 2.471 & 1.401 \\
\hline $\mathrm{H}$ & -2.327 & 1.095 & 2.514 \\
\hline $\mathrm{H}$ & -3.815 & 1.077 & 1.510 \\
\hline $\mathrm{Gd}$ & -0.372 & -2.673 & 1.069 \\
\hline \multicolumn{4}{|c|}{34} \\
\hline \multicolumn{4}{|c|}{ Structure 56, } \\
\hline $\mathrm{C}$ & 1.604 & -0.399 & -1.870 \\
\hline $\mathrm{C}$ & 2.852 & -0.341 & -0.979 \\
\hline $\mathrm{C}$ & 2.698 & 0.673 & 0.158 \\
\hline $\mathrm{P}$ & 1.324 & 0.177 & 1.220 \\
\hline $\mathrm{C}$ & -0.063 & 0.083 & 0.027 \\
\hline $\mathrm{C}$ & 0.316 & -0.864 & -1.148 \\
\hline $\mathrm{C}$ & -0.806 & -0.940 & -2.212 \\
\hline $\mathrm{C}$ & -2.150 & -1.464 & -1.688 \\
\hline $\mathrm{C}$ & -2.743 & -0.549 & -0.614 \\
\hline $\mathrm{P}$ & -1.623 & -0.485 & 0.802 \\
\hline $\mathrm{C}$ & 1.104 & 1.627 & 2.266 \\
\hline 0 & 1.593 & -1.078 & 1.980 \\
\hline $\mathrm{C}$ & -2.317 & 0.858 & 1.782 \\
\hline 0 & -1.531 & -1.780 & 1.537 \\
\hline $\mathrm{H}$ & 1.813 & -1.088 & -2.720 \\
\hline $\mathrm{H}$ & 1.433 & 0.606 & -2.321 \\
\hline $\mathrm{H}$ & 3.073 & -1.354 & -0.569 \\
\hline $\mathrm{H}$ & 3.732 & -0.058 & -1.601 \\
\hline $\mathrm{H}$ & 2.503 & 1.688 & -0.248 \\
\hline $\mathrm{H}$ & 3.626 & 0.728 & 0.766 \\
\hline $\mathrm{H}$ & -0.231 & 1.095 & -0.393 \\
\hline $\mathrm{H}$ & 0.490 & -1.892 & -0.750 \\
\hline $\mathrm{H}$ & -0.960 & 0.068 & -2.660 \\
\hline $\mathrm{H}$ & -0.473 & -1.602 & -3.044 \\
\hline $\mathrm{H}$ & -2.025 & -2.499 & -1.292 \\
\hline $\mathrm{H}$ & -2.868 & -1.540 & -2.537 \\
\hline $\mathrm{H}$ & -2.892 & 0.476 & -1.013 \\
\hline $\mathrm{H}$ & -3.732 & -0.925 & -0.277 \\
\hline $\mathrm{H}$ & 0.786 & 2.499 & 1.660 \\
\hline $\mathrm{H}$ & 0.341 & 1.430 & 3.044 \\
\hline $\mathrm{H}$ & 2.058 & 1.875 & 2.774 \\
\hline $\mathrm{H}$ & -2.246 & 1.818 & 1.230 \\
\hline $\mathrm{H}$ & -1.778 & 0.954 & 2.744 \\
\hline $\mathrm{H}$ & -3.385 & 0.653 & 2.003 \\
\hline \multicolumn{4}{|c|}{29} \\
\hline \multicolumn{4}{|c|}{ Str } \\
\hline $\mathrm{P}$ & 0.994 & 0.550 & -1.018 \\
\hline $\mathrm{C}$ & 0.207 & 1.756 & -2.126 \\
\hline $\mathrm{C}$ & -1.252 & 1.387 & -2.479 \\
\hline $\mathrm{C}$ & -2.239 & 1.321 & -1.290 \\
\hline $\mathrm{P}$ & -1.757 & 0.019 & -0.142 \\
\hline $\mathrm{C}$ & -0.156 & 0.709 & 0.373 \\
\hline $\mathrm{C}$ & 0.157 & -0.118 & 1.627 \\
\hline $\mathrm{C}$ & -1.142 & -0.141 & 2.471 \\
\hline $\mathrm{C}$ & -2.386 & 0.001 & 1.544 \\
\hline $\mathrm{C}$ & 2.589 & 1.207 & -0.524 \\
\hline 0 & 1.010 & -0.837 & -1.566 \\
\hline 0 & -1.601 & -1.322 & -0.776 \\
\hline $\mathrm{H}$ & 0.816 & 1.820 & -3.053 \\
\hline $\mathrm{H}$ & 0.242 & 2.757 & -1.647 \\
\hline $\mathrm{H}$ & -1.267 & 0.419 & -3.032 \\
\hline $\mathrm{H}$ & -1.635 & 2.146 & -3.201 \\
\hline $\mathrm{H}$ & -2.273 & 2.292 & -0.754 \\
\hline $\mathrm{H}$ & -3.267 & 1.107 & -1.651 \\
\hline $\mathrm{H}$ & -0.266 & 1.773 & 0.664 \\
\hline $\mathrm{H}$ & 1.004 & 0.319 & 2.204 \\
\hline $\mathrm{H}$ & 0.447 & -1.160 & 1.351 \\
\hline $\mathrm{H}$ & -1.199 & -1.082 & 3.066 \\
\hline $\mathrm{H}$ & -1.130 & 0.701 & 3.201 \\
\hline $\mathrm{H}$ & -3.102 & -0.836 & 1.675 \\
\hline $\mathrm{H}$ & -2.921 & 0.954 & 1.742 \\
\hline $\mathrm{H}$ & 2.471 & 2.226 & -0.104 \\
\hline $\mathrm{H}$ & 3.267 & 1.256 & -1.401 \\
\hline $\mathrm{H}$ & 3.047 & 0.553 & 0.246 \\
\hline
\end{tabular}




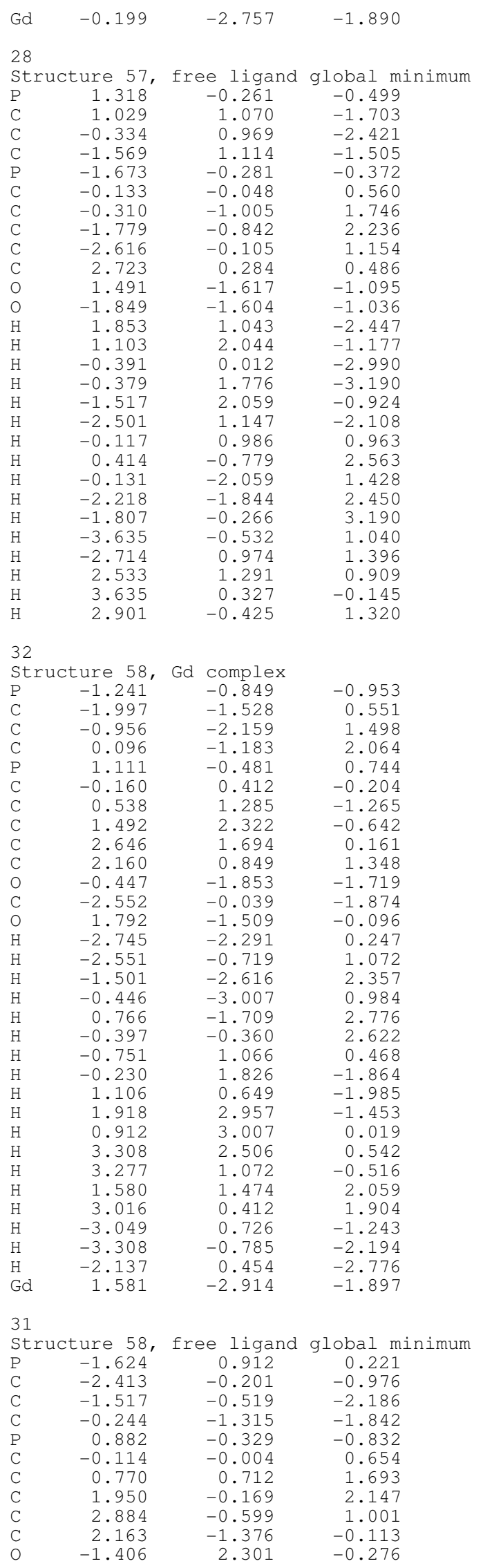




\begin{tabular}{|c|c|c|c|}
\hline $\mathrm{C}$ & -2.691 & 0.826 & 1.672 \\
\hline 0 & 1.418 & 0.879 & -1.524 \\
\hline $\mathrm{H}$ & -3.358 & 0.273 & -1.315 \\
\hline $\mathrm{H}$ & -2.693 & -1.142 & -0.456 \\
\hline $\mathrm{H}$ & -2.114 & -1.117 & -2.913 \\
\hline $\mathrm{H}$ & -1.249 & 0.423 & -2.721 \\
\hline $\mathrm{H}$ & 0.289 & -1.614 & -2.768 \\
\hline $\mathrm{H}$ & -0.504 & -2.246 & -1.296 \\
\hline $\mathrm{H}$ & -0.423 & -0.977 & 1.086 \\
\hline $\mathrm{H}$ & 0.160 & 0.974 & 2.588 \\
\hline $\mathrm{H}$ & 1.155 & 1.675 & 1.283 \\
\hline $\mathrm{H}$ & 2.543 & 0.382 & 2.913 \\
\hline $\mathrm{H}$ & 1.553 & -1.078 & 2.655 \\
\hline $\mathrm{H}$ & 3.698 & -1.237 & 1.417 \\
\hline $\mathrm{H}$ & 3.386 & 0.300 & 0.573 \\
\hline $\mathrm{H}$ & 1.701 & -2.301 & 0.290 \\
\hline $\mathrm{H}$ & 2.874 & -1.677 & -0.911 \\
\hline $\mathrm{H}$ & -2.790 & -0.223 & 2.018 \\
\hline $\mathrm{H}$ & -3.698 & 1.217 & 1.425 \\
\hline $\mathrm{H}$ & -2.267 & 1.435 & 2.496 \\
\hline \multicolumn{4}{|c|}{32} \\
\hline \multicolumn{4}{|c|}{ Structure 59, Gd complex } \\
\hline $\mathrm{C}$ & 0.446 & 0.679 & 1.218 \\
\hline $\mathrm{P}$ & 1.632 & 0.131 & -0.033 \\
\hline $\mathrm{C}$ & 1.484 & 1.431 & -1.271 \\
\hline $\mathrm{C}$ & 0.018 & 1.530 & -1.715 \\
\hline $\mathrm{C}$ & -0.919 & 2.002 & -0.582 \\
\hline $\mathrm{C}$ & -0.935 & 1.085 & 0.664 \\
\hline $\mathrm{C}$ & -2.389 & 2.035 & -1.057 \\
\hline $\mathrm{P}$ & -2.093 & -0.273 & 0.272 \\
\hline $\mathrm{C}$ & -2.934 & 0.594 & -1.078 \\
\hline $\mathrm{C}$ & 3.265 & 0.306 & 0.701 \\
\hline O & 1.348 & -1.233 & -0.558 \\
\hline 0 & -1.466 & -1.563 & -0.130 \\
\hline $\mathrm{C}$ & -3.235 & -0.389 & 1.655 \\
\hline $\mathrm{Gd}$ & 0.037 & -3.029 & -1.020 \\
\hline $\mathrm{H}$ & 0.896 & 1.558 & 1.727 \\
\hline $\mathrm{H}$ & 0.346 & -0.113 & 1.989 \\
\hline $\mathrm{H}$ & 1.832 & 2.395 & -0.843 \\
\hline $\mathrm{H}$ & 2.143 & 1.186 & -2.131 \\
\hline $\mathrm{H}$ & -0.320 & 0.551 & -2.124 \\
\hline $\mathrm{H}$ & -0.049 & 2.254 & -2.560 \\
\hline $\mathrm{H}$ & -0.606 & 3.029 & -0.278 \\
\hline $\mathrm{H}$ & -1.423 & 1.673 & 1.472 \\
\hline $\mathrm{H}$ & -2.992 & 2.649 & -0.349 \\
\hline $\mathrm{H}$ & -2.483 & 2.515 & -2.059 \\
\hline $\mathrm{H}$ & -2.685 & 0.091 & -2.036 \\
\hline $\mathrm{H}$ & -4.035 & 0.559 & -0.947 \\
\hline $\mathrm{H}$ & 3.417 & 1.341 & 1.068 \\
\hline $\mathrm{H}$ & 4.046 & 0.076 & -0.053 \\
\hline $\mathrm{H}$ & 3.375 & -0.395 & 1.553 \\
\hline $\mathrm{H}$ & -2.699 & -0.743 & 2.560 \\
\hline $\mathrm{H}$ & -4.046 & -1.107 & 1.417 \\
\hline $\mathrm{H}$ & -3.686 & 0.601 & 1.870 \\
\hline \multicolumn{4}{|c|}{31} \\
\hline \multicolumn{4}{|c|}{ Structure 59, } \\
\hline $\mathrm{C}$ & 1.134 & -0.597 & -0.488 \\
\hline $\mathrm{P}$ & 2.105 & 0.925 & -0.419 \\
\hline $\mathrm{C}$ & 0.862 & 2.175 & -0.803 \\
\hline $\mathrm{C}$ & -0.377 & 2.034 & 0.089 \\
\hline $\mathrm{C}$ & -1.074 & 0.661 & 0.049 \\
\hline $\mathrm{C}$ & -0.153 & -0.542 & 0.365 \\
\hline $\mathrm{C}$ & -1.750 & 0.339 & -1.298 \\
\hline $\mathrm{P}$ & -1.209 & -1.979 & -0.043 \\
\hline $\mathrm{C}$ & -2.256 & -1.120 & -1.249 \\
\hline $\mathrm{C}$ & 2.602 & 1.152 & 1.298 \\
\hline 0 & 3.261 & 0.938 & -1.361 \\
\hline 0 & -0.486 & -3.179 & -0.551 \\
\hline $\mathrm{C}$ & -2.236 & -2.285 & 1.404 \\
\hline $\mathrm{H}$ & 0.895 & -0.785 & -1.555 \\
\hline $\mathrm{H}$ & 1.784 & -1.433 & -0.153 \\
\hline $\mathrm{H}$ & 0.585 & 2.082 & -1.873 \\
\hline $\mathrm{H}$ & 1.319 & 3.179 & -0.674 \\
\hline $\mathrm{H}$ & -1.116 & 2.817 & -0.201 \\
\hline $\mathrm{H}$ & -0.099 & 2.266 & 1.143 \\
\hline $\mathrm{H}$ & -1.879 & 0.691 & 0.822 \\
\hline $\mathrm{H}$ & 0.112 & -0.552 & 1.444 \\
\hline $\mathrm{H}$ & -1.023 & 0.454 & -2.133 \\
\hline $\mathrm{H}$ & -2.591 & 1.042 & -1.502 \\
\hline
\end{tabular}




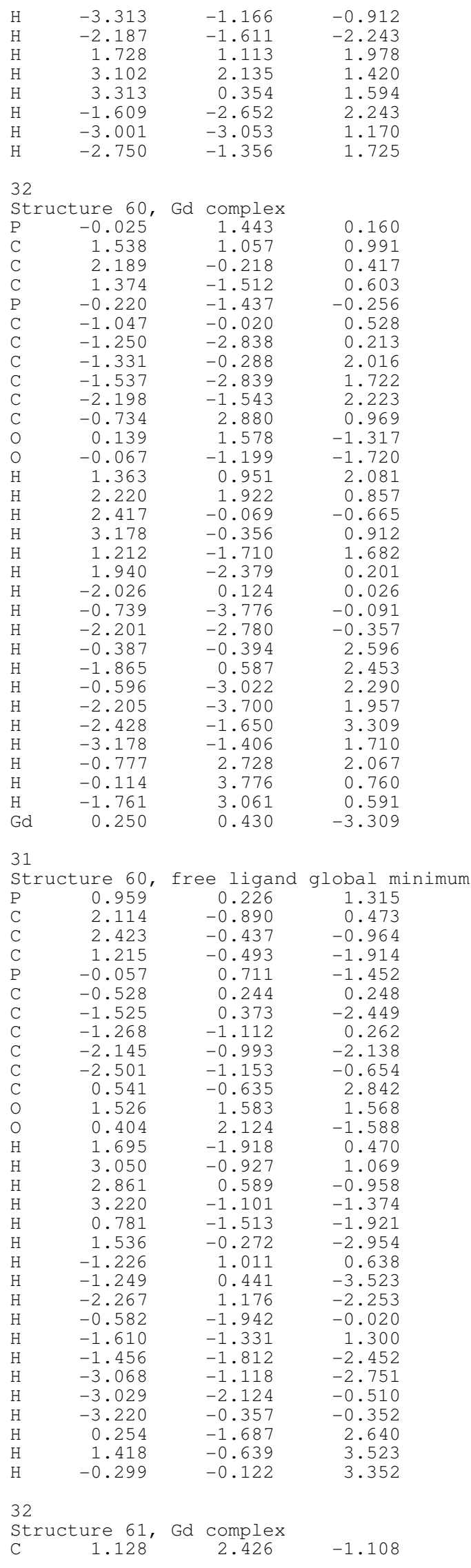




\begin{tabular}{|c|c|c|c|}
\hline $\mathrm{C}$ & 2.516 & 1.790 & -0.887 \\
\hline $\mathrm{C}$ & 2.586 & 0.894 & 0.360 \\
\hline $\mathrm{P}$ & 1.350 & -0.427 & 0.313 \\
\hline $\mathrm{C}$ & -0.169 & 0.496 & -0.064 \\
\hline $\mathrm{C}$ & 0.055 & 1.350 & -1.329 \\
\hline $\mathrm{C}$ & -1.341 & 1.890 & -1.679 \\
\hline $\mathrm{C}$ & -2.315 & 0.687 & -1.671 \\
\hline $\mathrm{P}$ & -1.585 & -0.546 & -0.555 \\
\hline 0 & 1.581 & -1.527 & -0.665 \\
\hline $\mathrm{C}$ & 1.190 & -1.000 & 2.012 \\
\hline $\mathrm{C}$ & -2.651 & -0.755 & 0.873 \\
\hline $\mathrm{O}$ & -1.143 & -1.807 & -1.219 \\
\hline $\mathrm{H}$ & 1.168 & 3.095 & -1.998 \\
\hline $\mathrm{H}$ & 0.858 & 3.066 & -0.237 \\
\hline $\mathrm{H}$ & 3.276 & 2.601 & -0.793 \\
\hline $\mathrm{H}$ & 2.803 & 1.201 & -1.789 \\
\hline $\mathrm{H}$ & 2.412 & 1.506 & 1.270 \\
\hline $\mathrm{H}$ & 3.593 & 0.437 & 0.460 \\
\hline $\mathrm{H}$ & -0.448 & 1.152 & 0.785 \\
\hline $\mathrm{H}$ & 0.389 & 0.699 & -2.173 \\
\hline $\mathrm{H}$ & -1.659 & 2.641 & -0.919 \\
\hline $\mathrm{H}$ & -1.343 & 2.399 & -2.670 \\
\hline $\mathrm{H}$ & -2.403 & 0.241 & -2.684 \\
\hline $\mathrm{H}$ & -3.330 & 0.978 & -1.329 \\
\hline $\mathrm{H}$ & 0.940 & -0.154 & 2.684 \\
\hline $\mathrm{H}$ & 0.387 & -1.762 & 2.081 \\
\hline $\mathrm{H}$ & 2.142 & -1.456 & 2.349 \\
\hline $\mathrm{H}$ & -2.897 & 0.231 & 1.317 \\
\hline $\mathrm{H}$ & -3.593 & -1.258 & 0.573 \\
\hline $\mathrm{H}$ & -2.144 & -1.376 & 1.639 \\
\hline $\mathrm{Gd}$ & 0.590 & -3.095 & -2.002 \\
\hline \multicolumn{4}{|c|}{31} \\
\hline \multicolumn{4}{|c|}{ Structure 61, } \\
\hline $\mathrm{C}$ & 0.369 & -1.228 & -1.835 \\
\hline C & 1.796 & -0.667 & -2.016 \\
\hline $\mathrm{C}$ & 2.004 & 0.727 & -1.392 \\
\hline $\mathrm{P}$ & 1.654 & 0.705 & 0.389 \\
\hline $\mathrm{C}$ & -0.050 & 0.091 & 0.285 \\
\hline $\mathrm{C}$ & -0.024 & $-1 \cdot 314$ & -0.351 \\
\hline $\mathrm{C}$ & -1.417 & -1.908 & -0.085 \\
\hline $\mathrm{C}$ & -1.690 & -1.723 & 1.420 \\
\hline $\mathrm{P}$ & -0.988 & -0.101 & 1.835 \\
\hline 0 & 2.594 & -0.147 & 1.174 \\
\hline $\mathrm{C}$ & 1.576 & 2.422 & 0.918 \\
\hline $\mathrm{C}$ & -2.345 & 1.077 & 1.726 \\
\hline $\mathrm{O}$ & -0.213 & -0.034 & 3.105 \\
\hline $\mathrm{H}$ & 0.310 & -2.242 & -2.294 \\
\hline $\mathrm{H}$ & -0.359 & -0.588 & -2.385 \\
\hline $\mathrm{H}$ & 2.028 & -0.613 & -3.105 \\
\hline $\mathrm{H}$ & 2.531 & -1.383 & -1.581 \\
\hline $\mathrm{H}$ & 1.340 & 1.470 & -1.882 \\
\hline $\mathrm{H}$ & 3.050 & 1.070 & -1.544 \\
\hline $\mathrm{H}$ & -0.628 & 0.764 & -0.380 \\
\hline $\mathrm{H}$ & 0.722 & -1.957 & 0.176 \\
\hline $\mathrm{H}$ & -2.186 & -1.372 & -0.687 \\
\hline $\mathrm{H}$ & -1.460 & -2.985 & -0.370 \\
\hline $\mathrm{H}$ & -1.165 & -2.502 & 2.012 \\
\hline $\mathrm{H}$ & -2.772 & -1.766 & 1.665 \\
\hline $\mathrm{H}$ & 0.852 & 2.985 & 0.294 \\
\hline $\mathrm{H}$ & 1.256 & 2.481 & 1.978 \\
\hline $\mathrm{H}$ & 2.575 & 2.895 & 0.821 \\
\hline $\mathrm{H}$ & -2.896 & 0.949 & 0.772 \\
\hline $\mathrm{H}$ & -3.050 & 0.923 & 2.568 \\
\hline $\mathrm{H}$ & -1.952 & 2.113 & 1.776 \\
\hline \multicolumn{4}{|c|}{32} \\
\hline \multicolumn{4}{|c|}{ St } \\
\hline $\mathrm{P}$ & 0.763 & 0.568 & -1.424 \\
\hline $\mathrm{C}$ & 1.747 & 1.063 & 0.008 \\
\hline $\mathrm{P}$ & 1.601 & -0.234 & 1.257 \\
\hline $\mathrm{C}$ & -0.141 & -0.012 & 1.694 \\
\hline $\mathrm{C}$ & -1.103 & -0.313 & 0.519 \\
\hline $\mathrm{C}$ & -0.927 & 0.552 & -0.759 \\
\hline $\mathrm{C}$ & -2.545 & 0.065 & 0.933 \\
\hline $\mathrm{C}$ & -1.524 & 1.917 & -0.354 \\
\hline $\mathrm{C}$ & -2.675 & 1.580 & 0.634 \\
\hline $\mathrm{O}$ & 1.184 & -0.784 & -1.892 \\
\hline $\mathrm{C}$ & 0.883 & 1.865 & -2.660 \\
\hline $\mathrm{C}$ & 2.596 & 0.243 & 2.673 \\
\hline 0 & 1.906 & -1.575 & 0.680 \\
\hline
\end{tabular}




\begin{tabular}{|c|c|c|c|}
\hline $\mathrm{Gd}$ & 1.951 & -2.845 & -1.234 \\
\hline $\mathrm{H}$ & 1.410 & 2.038 & 0.409 \\
\hline $\mathrm{H}$ & 2.808 & 1.159 & -0.295 \\
\hline $\mathrm{H}$ & -0.366 & -0.687 & 2.545 \\
\hline $\mathrm{H}$ & -0.279 & 1.025 & 2.063 \\
\hline $\mathrm{H}$ & -1.049 & -1.399 & 0.264 \\
\hline $\mathrm{H}$ & -1.587 & 0.125 & -1.545 \\
\hline $\mathrm{H}$ & -2.756 & -0.171 & 2.001 \\
\hline $\mathrm{H}$ & -3.276 & -0.504 & 0.313 \\
\hline $\mathrm{H}$ & -0.769 & 2.557 & 0.155 \\
\hline $\mathrm{H}$ & -1.900 & 2.478 & -1.241 \\
\hline $\mathrm{H}$ & -2.573 & 2.180 & 1.567 \\
\hline $\mathrm{H}$ & -3.671 & 1.810 & 0.188 \\
\hline $\mathrm{H}$ & 0.609 & 2.845 & -2.220 \\
\hline $\mathrm{H}$ & 1.921 & 1.926 & -3.045 \\
\hline $\mathrm{H}$ & 0.200 & 1.647 & -3.506 \\
\hline $\mathrm{H}$ & 2.314 & 1.260 & 3.015 \\
\hline $\mathrm{H}$ & 2.439 & -0.472 & 3.506 \\
\hline $\begin{array}{l}\mathrm{H} \\
31\end{array}$ & 3.671 & 0.243 & 2.398 \\
\hline St & ture 62, & free ligand & global minimum \\
\hline $\mathrm{P}$ & -0.802 & 0.922 & -1.335 \\
\hline $\mathrm{C}$ & 0.547 & 1.543 & -0.309 \\
\hline $\mathrm{P}$ & 1.711 & 0.239 & 0.141 \\
\hline $\mathrm{C}$ & 0.642 & -0.939 & 0.998 \\
\hline $\mathrm{C}$ & -0.484 & -1.510 & 0.108 \\
\hline $\mathrm{C}$ & -1.512 & -0.477 & -0.419 \\
\hline $\mathrm{C}$ & -1.395 & -2.414 & 0.973 \\
\hline $\mathrm{C}$ & -2.331 & -0.128 & 0.842 \\
\hline $\mathrm{C}$ & -2.395 & -1.447 & 1.660 \\
\hline 0 & -0.383 & 0.561 & -2.720 \\
\hline $\mathrm{C}$ & -2.039 & 2.232 & -1.306 \\
\hline $\mathrm{C}$ & 2.745 & 0.963 & 1.426 \\
\hline 0 & 2.484 & -0.344 & -0.993 \\
\hline $\mathrm{H}$ & 0.126 & 1.984 & 0.615 \\
\hline $\mathrm{H}$ & 1.084 & 2.344 & -0.853 \\
\hline $\mathrm{H}$ & 1.282 & -1.765 & 1.372 \\
\hline $\mathrm{H}$ & 0.220 & -0.437 & 1.893 \\
\hline $\mathrm{H}$ & -0.042 & -2.096 & -0.734 \\
\hline $\mathrm{H}$ & -2.197 & -1.010 & -1.114 \\
\hline $\mathrm{H}$ & -0.819 & -3.016 & 1.713 \\
\hline $\mathrm{H}$ & -1.949 & -3.127 & 0.319 \\
\hline $\mathrm{H}$ & -1.829 & 0.665 & 1.442 \\
\hline $\mathrm{H}$ & -3.352 & 0.237 & 0.582 \\
\hline $\mathrm{H}$ & -2.107 & -1.263 & 2.720 \\
\hline $\mathrm{H}$ & -3.423 & -1.877 & 1.655 \\
\hline $\mathrm{H}$ & -2.285 & 2.520 & -0.264 \\
\hline $\mathrm{H}$ & -1.654 & 3.127 & -1.836 \\
\hline $\mathrm{H}$ & -2.966 & 1.891 & -1.812 \\
\hline $\mathrm{H}$ & 2.119 & 1.373 & 2.244 \\
\hline $\mathrm{H}$ & 3.423 & 0.192 & 1.847 \\
\hline $\mathrm{H}$ & 3.359 & 1.783 & 1.001 \\
\hline \multicolumn{4}{|l|}{29} \\
\hline St & ture & Gd complex & \\
\hline $\mathrm{C}$ & -0.173 & 1.495 & -1.526 \\
\hline $\mathrm{C}$ & -1.603 & 1.382 & -0.948 \\
\hline $\mathrm{P}$ & -1.832 & -0.125 & 0.041 \\
\hline $\mathrm{C}$ & -0.556 & 0.093 & 1.313 \\
\hline $\mathrm{P}$ & 1.044 & 0.008 & 0.494 \\
\hline $\mathrm{C}$ & 0.917 & 1.563 & -0.441 \\
\hline $\mathrm{C}$ & 2.356 & 1.745 & -0.941 \\
\hline $\mathrm{C}$ & 3.284 & 1.470 & 0.272 \\
\hline $\mathrm{C}$ & 2.568 & 0.533 & 1.290 \\
\hline O & -1.590 & -1.368 & -0.747 \\
\hline $\mathrm{C}$ & -3.434 & -0.020 & 0.843 \\
\hline 0 & 1.155 & -1.205 & -0.367 \\
\hline $\mathrm{H}$ & -0.114 & 2.420 & -2.146 \\
\hline $\mathrm{H}$ & 0.025 & 0.648 & -2.223 \\
\hline $\mathrm{H}$ & -1.830 & 2.263 & -0.312 \\
\hline $\mathrm{H}$ & -2.348 & 1.373 & -1.771 \\
\hline $\mathrm{H}$ & -0.632 & -0.722 & 2.058 \\
\hline $\mathrm{H}$ & -0.683 & 1.062 & 1.833 \\
\hline $\mathrm{H}$ & 0.682 & 2.400 & 0.252 \\
\hline $\mathrm{H}$ & 2.518 & 2.769 & -1.349 \\
\hline $\mathrm{H}$ & 2.575 & 1.023 & -1.762 \\
\hline $\mathrm{H}$ & 4.241 & 1.017 & -0.074 \\
\hline $\mathrm{H}$ & 3.543 & 2.430 & 0.775 \\
\hline $\mathrm{H}$ & 3.187 & -0.350 & 1.556 \\
\hline $\mathrm{H}$ & 2.318 & 1.079 & 2.223 \\
\hline $\mathrm{H}$ & -3.515 & 0.925 & 1.418 \\
\hline
\end{tabular}

S-82 


\begin{tabular}{|c|c|c|c|}
\hline $\begin{array}{l}\mathrm{H} \\
\mathrm{H} \\
\mathrm{Gd}\end{array}$ & $\begin{array}{r}-3.567 \\
-4.241 \\
0.014\end{array}$ & $\begin{array}{l}-0.874 \\
-0.048 \\
-2.769\end{array}$ & $\begin{array}{r}1.538 \\
0.083 \\
-1.603\end{array}$ \\
\hline \multicolumn{4}{|l|}{28} \\
\hline \multicolumn{2}{|c|}{ Structure 63, } & free ligand & \multirow{2}{*}{$\begin{array}{l}\text { global minimum } \\
-0.133\end{array}$} \\
\hline $\mathrm{C}$ & 1.264 & 1.733 & \\
\hline C & -0.216 & 2.165 & -0.184 \\
\hline $\mathrm{P}$ & -1.289 & 0.971 & -1.037 \\
\hline $\mathrm{C}$ & -0.947 & -0.630 & -0.254 \\
\hline $\mathrm{P}$ & 0.811 & -1.004 & -0.279 \\
\hline $\mathrm{C}$ & 1.451 & 0.421 & 0.646 \\
\hline $\mathrm{C}$ & 2.894 & -0.009 & 0.943 \\
\hline $\mathrm{C}$ & 2.827 & -1.491 & 1.412 \\
\hline $\mathrm{C}$ & 1.475 & -2.121 & 0.971 \\
\hline 0 & -1.138 & 0.975 & -2.521 \\
\hline $\mathrm{C}$ & -2.955 & 1.387 & -0.491 \\
\hline 0 & 1.402 & -1.201 & -1.634 \\
\hline $\mathrm{H}$ & 1.851 & 2.535 & 0.372 \\
\hline $\mathrm{H}$ & 1.682 & 1.646 & -1.162 \\
\hline $\mathrm{H}$ & -0.600 & 2.305 & 0.848 \\
\hline $\mathrm{H}$ & -0.312 & 3.145 & -0.697 \\
\hline $\mathrm{H}$ & -1.506 & -1.429 & -0.779 \\
\hline $\mathrm{H}$ & -1.294 & -0.603 & 0.797 \\
\hline $\mathrm{H}$ & 0.915 & 0.507 & 1.616 \\
\hline $\mathrm{H}$ & 3.358 & 0.640 & 1.722 \\
\hline $\mathrm{H}$ & 3.520 & 0.076 & 0.025 \\
\hline $\mathrm{H}$ & 3.682 & -2.062 & 0.982 \\
\hline $\mathrm{H}$ & 2.923 & -1.550 & 2.521 \\
\hline $\mathrm{H}$ & 1.598 & -3.145 & 0.558 \\
\hline $\mathrm{H}$ & 0.766 & -2.171 & 1.824 \\
\hline $\mathrm{H}$ & -3.017 & 1.370 & 0.617 \\
\hline $\mathrm{H}$ & -3.682 & 0.655 & -0.899 \\
\hline $\mathrm{H}$ & -3.230 & 2.400 & -0.848 \\
\hline \multicolumn{4}{|c|}{32} \\
\hline \multicolumn{2}{|r|}{ Eure 64, } & Gd complex & \\
\hline $\mathrm{C}$ & -2.494 & -0.227 & -0.657 \\
\hline C & -2.727 & 1.085 & 0.103 \\
\hline C & -2.102 & 2.306 & -0.592 \\
\hline $\mathrm{C}$ & -0.569 & 2.230 & -0.689 \\
\hline $\mathrm{C}$ & -0.076 & 1.043 & -1.540 \\
\hline $\mathrm{P}$ & -0.738 & -0.534 & -0.913 \\
\hline C & 1.469 & 0.996 & -1.644 \\
\hline $\mathrm{C}$ & 0.114 & -0.705 & 0.672 \\
\hline $\mathrm{C}$ & 2.256 & 0.714 & -0.347 \\
\hline $\mathrm{P}$ & 1.877 & -0.920 & 0.339 \\
\hline 0 & -0.413 & -1.688 & -1.800 \\
\hline 0 & 2.102 & -2.034 & -0.628 \\
\hline $\mathrm{C}$ & 2.728 & -1.078 & 1.911 \\
\hline $\mathrm{H}$ & -2.991 & -0.188 & -1.649 \\
\hline $\mathrm{H}$ & -2.930 & -1.085 & -0.103 \\
\hline $\mathrm{H}$ & -3.825 & 1.250 & 0.207 \\
\hline $\mathrm{H}$ & -2.330 & 1.002 & 1.142 \\
\hline $\mathrm{H}$ & -2.385 & 3.227 & -0.031 \\
\hline $\mathrm{H}$ & -2.536 & 2.416 & -1.613 \\
\hline $\mathrm{H}$ & -0.191 & 3.177 & -1.139 \\
\hline $\mathrm{H}$ & -0.139 & 2.188 & 0.336 \\
\hline $\mathrm{H}$ & -0.474 & 1.188 & -2.569 \\
\hline $\mathrm{H}$ & 1.757 & 0.240 & -2.412 \\
\hline $\mathrm{H}$ & 1.818 & 1.972 & -2.054 \\
\hline $\mathrm{H}$ & -0.271 & -1.601 & 1.196 \\
\hline $\mathrm{H}$ & -0.053 & 0.175 & 1.322 \\
\hline $\mathrm{H}$ & 3.347 & 0.753 & -0.552 \\
\hline $\mathrm{H}$ & 2.047 & 1.488 & 0.419 \\
\hline $\mathrm{H}$ & 2.482 & -0.219 & 2.569 \\
\hline $\mathrm{H}$ & 2.421 & -2.016 & 2.416 \\
\hline $\mathrm{H}$ & 3.825 & -1.102 & 1.748 \\
\hline $\mathrm{Gd}$ & 1.194 & -3.227 & -2.369 \\
\hline \multicolumn{4}{|l|}{31} \\
\hline \multicolumn{2}{|c|}{ Str } & free ligand & global minimum \\
\hline $\mathrm{C}$ & $-2.799^{\circ}$ & -1.149 & 0.704 \\
\hline $\mathrm{C}$ & -2.866 & 0.123 & 1.557 \\
\hline C & -2.663 & 1.404 & 0.735 \\
\hline $\mathrm{C}$ & -1.267 & 1.479 & 0.098 \\
\hline C & -1.009 & 0.367 & -0.940 \\
\hline $\mathrm{P}$ & -1.253 & -1.289 & -0.223 \\
\hline $\mathrm{C}$ & 0.397 & 0.489 & -1.574 \\
\hline $\mathrm{C}$ & 0.100 & -1.464 & 0.961 \\
\hline $\mathrm{C}$ & 1.591 & 0.392 & -0.606 \\
\hline
\end{tabular}

S-83 


\begin{tabular}{|c|c|c|c|}
\hline $\mathrm{P}$ & 1.719 & -1.229 & 0.192 \\
\hline 0 & -1.296 & -2.377 & -1.243 \\
\hline 0 & 2.140 & -2.328 & -0.724 \\
\hline $\mathrm{C}$ & 2.849 & -0.977 & 1.573 \\
\hline $\mathrm{H}$ & -3.638 & -1.157 & -0.024 \\
\hline $\mathrm{H}$ & -2.913 & -2.052 & 1.340 \\
\hline $\mathrm{H}$ & -3.861 & 0.167 & 2.059 \\
\hline $\mathrm{H}$ & -2.112 & 0.078 & 2.376 \\
\hline $\mathrm{H}$ & -2.810 & 2.289 & 1.396 \\
\hline $\mathrm{H}$ & -3.443 & 1.471 & -0.059 \\
\hline $\mathrm{H}$ & -1.150 & 2.470 & -0.398 \\
\hline $\mathrm{H}$ & -0.504 & 1.449 & 0.907 \\
\hline $\mathrm{H}$ & -1.756 & 0.494 & -1.753 \\
\hline $\mathrm{H}$ & 0.510 & -0.278 & -2.376 \\
\hline $\mathrm{H}$ & 0.457 & 1.471 & -2.098 \\
\hline $\mathrm{H}$ & 0.056 & -2.470 & 1.422 \\
\hline $\mathrm{H}$ & -0.020 & -0.720 & 1.770 \\
\hline $\mathrm{H}$ & 2.538 & 0.576 & -1.155 \\
\hline $\mathrm{H}$ & 1.526 & 1.176 & 0.176 \\
\hline $\mathrm{H}$ & 2.493 & -0.149 & 2.220 \\
\hline $\mathrm{H}$ & 2.916 & -1.902 & 2.182 \\
\hline $\mathrm{H}$ & 3.861 & -0.725 & 1.195 \\
\hline \multicolumn{4}{|c|}{32} \\
\hline \multicolumn{4}{|c|}{ Structure 65, Gd complex } \\
\hline $\mathrm{C}$ & -0.221 & 1.315 & -1.638 \\
\hline $\mathrm{C}$ & -1.567 & 1.743 & -1.016 \\
\hline $\mathrm{P}$ & -2.139 & 0.579 & 0.252 \\
\hline $\mathrm{C}$ & -0.770 & 0.702 & 1.430 \\
\hline $\mathrm{P}$ & 0.699 & 0.019 & 0.637 \\
\hline $\mathrm{C}$ & 0.968 & 1.280 & -0.649 \\
\hline $\mathrm{C}$ & 2.298 & 0.965 & -1.360 \\
\hline $\mathrm{C}$ & 3.504 & 1.000 & -0.402 \\
\hline $\mathrm{C}$ & 3.417 & -0.023 & 0.746 \\
\hline $\mathrm{C}$ & 2.183 & 0.177 & 1.639 \\
\hline $\mathrm{C}$ & -3.604 & 1.271 & 1.025 \\
\hline 0 & -2.280 & -0.815 & -0.260 \\
\hline 0 & 0.432 & -1.343 & 0.090 \\
\hline $\mathrm{Gd}$ & -1.159 & -2.754 & -0.773 \\
\hline $\mathrm{H}$ & 0.023 & 2.033 & -2.454 \\
\hline $\mathrm{H}$ & -0.333 & 0.323 & -2.135 \\
\hline $\mathrm{H}$ & -1.479 & 2.754 & -0.564 \\
\hline $\mathrm{H}$ & -2.347 & 1.806 & -1.803 \\
\hline $\mathrm{H}$ & -1.008 & 0.110 & 2.335 \\
\hline $\mathrm{H}$ & -0.602 & 1.755 & 1.728 \\
\hline $\mathrm{H}$ & 1.056 & 2.280 & -0.172 \\
\hline $\mathrm{H}$ & 2.464 & 1.710 & -2.173 \\
\hline $\mathrm{H}$ & 2.243 & -0.031 & -1.857 \\
\hline $\mathrm{H}$ & 4.436 & 0.814 & -0.984 \\
\hline $\mathrm{H}$ & 3.604 & 2.024 & 0.026 \\
\hline $\mathrm{H}$ & 4.335 & 0.057 & 1.374 \\
\hline $\mathrm{H}$ & 3.419 & -1.056 & 0.325 \\
\hline $\mathrm{H}$ & 2.200 & 1.183 & 2.110 \\
\hline $\mathrm{H}$ & 2.153 & -0.576 & 2.454 \\
\hline $\mathrm{H}$ & -3.397 & 2.297 & 1.390 \\
\hline $\mathrm{H}$ & -4.436 & 1.311 & 0.293 \\
\hline $\mathrm{H}$ & -3.913 & 0.640 & 1.884 \\
\hline \multicolumn{4}{|c|}{31} \\
\hline & cure 65 , & free ligand & global minimum \\
\hline $\mathrm{C}$ & 0.180 & 0.217 & 1.950 \\
\hline $\mathrm{C}$ & -1.345 & 0.036 & 1.825 \\
\hline $\mathrm{P}$ & -2.042 & 0.865 & 0.372 \\
\hline $\mathrm{C}$ & -1.080 & 0.210 & -1.014 \\
\hline $\mathrm{P}$ & 0.692 & 0.426 & -0.762 \\
\hline $\mathrm{C}$ & 0.967 & -0.457 & 0.806 \\
\hline $\mathrm{C}$ & 2.482 & -0.505 & 1.075 \\
\hline $\mathrm{C}$ & 3.240 & -1.294 & -0.010 \\
\hline $\mathrm{C}$ & 3.077 & -0.718 & -1.428 \\
\hline $\mathrm{C}$ & 1.611 & -0.643 & -1.887 \\
\hline $\mathrm{C}$ & -3.681 & 0.144 & 0.170 \\
\hline O & -2.059 & 2.353 & 0.469 \\
\hline 0 & 1.156 & 1.843 & -0.770 \\
\hline $\mathrm{H}$ & 0.502 & -0.235 & 2.917 \\
\hline $\mathrm{H}$ & 0.438 & 1.299 & 2.020 \\
\hline $\mathrm{H}$ & -1.593 & -1.045 & 1.771 \\
\hline $\mathrm{H}$ & -1.853 & 0.437 & 2.728 \\
\hline $\mathrm{H}$ & -1.386 & 0.716 & -1.951 \\
\hline $\mathrm{H}$ & -1.296 & -0.870 & -1.123 \\
\hline $\mathrm{H}$ & 0.603 & -1.502 & 0.707 \\
\hline $\mathrm{H}$ & 2.665 & -0.989 & 2.063 \\
\hline
\end{tabular}




$\begin{array}{rrr}2.896 & 0.527 & 1.152 \\ 4.324 & -1.323 & 0.250 \\ 2.891 & -2.353 & -0.003 \\ 3.648 & -1.353 & -2.146 \\ 3.542 & 0.294 & -1.474 \\ 1.151 & -1.653 & -1.895 \\ 1.538 & -0.232 & -2.917 \\ -3.614 & -0.962 & 0.105 \\ -4.324 & 0.413 & 1.033 \\ -4.153 & 0.527 & -0.758\end{array}$

S-85 


\section{(6) Metal size preference scans}

Figures $\mathrm{S} 7$ through $\mathrm{S} 11$ show plots of $\mathrm{U}(\mathrm{M})-\mathrm{U}(\mathrm{Gd})$ versus the $\mathrm{M}-\mathrm{O}$ distance for $\mathrm{R}=\mathrm{Me}$ analogs of $\mathbf{1}-\mathbf{6 5}$. In almost all cases, the curve shows a smooth monotonic trend. The two cases that do not, $\mathbf{2 2}$ and $\mathbf{2 8}$, are caused by the ligand undergoing a conformational change during the scan.

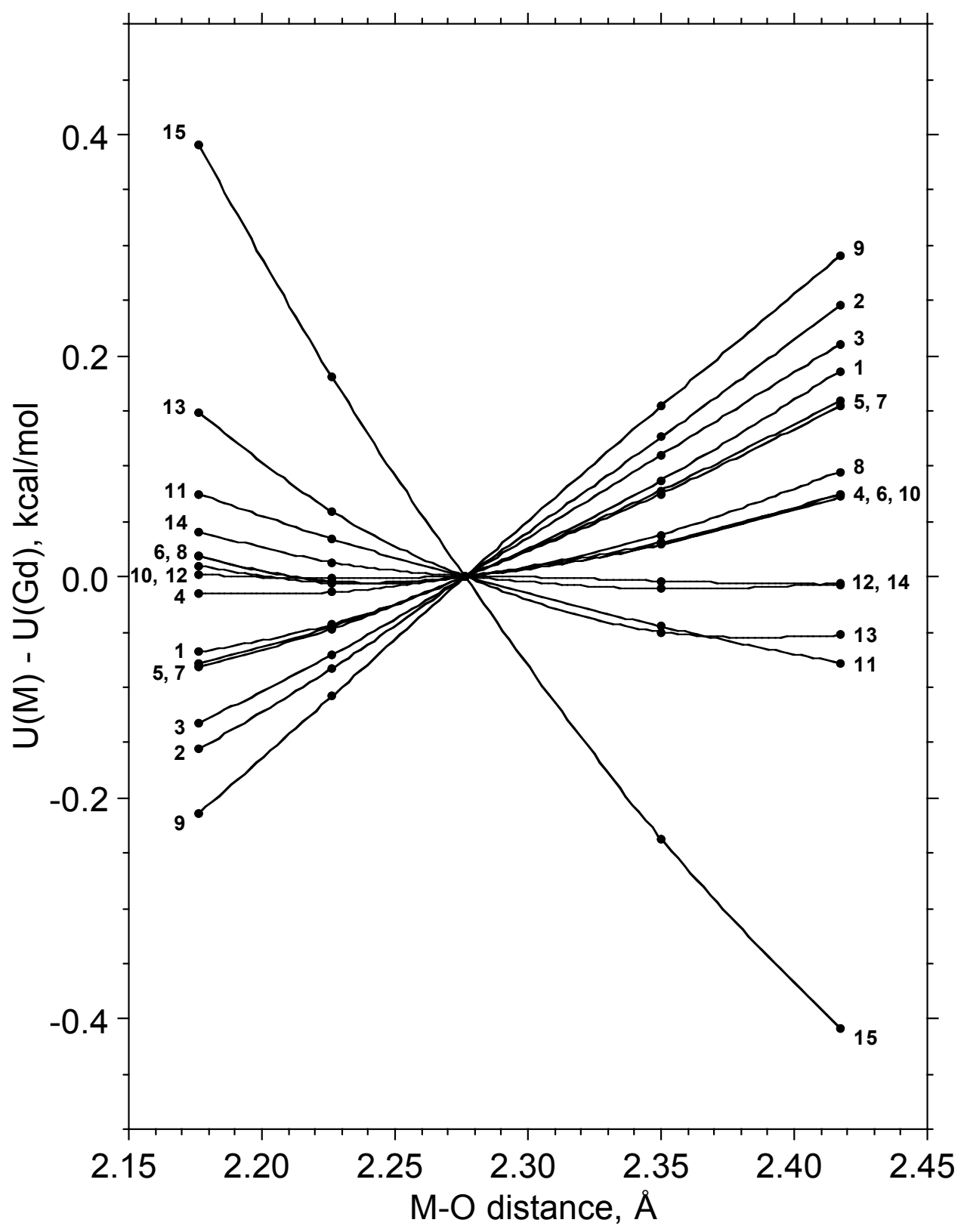

Figure S7. $\mathrm{U}(\mathrm{M})$ - $\mathrm{U}(\mathrm{Gd})$ versus $\mathrm{M}-\mathrm{O}$ distance for $\mathbf{1} \mathbf{- 1 5}$. 


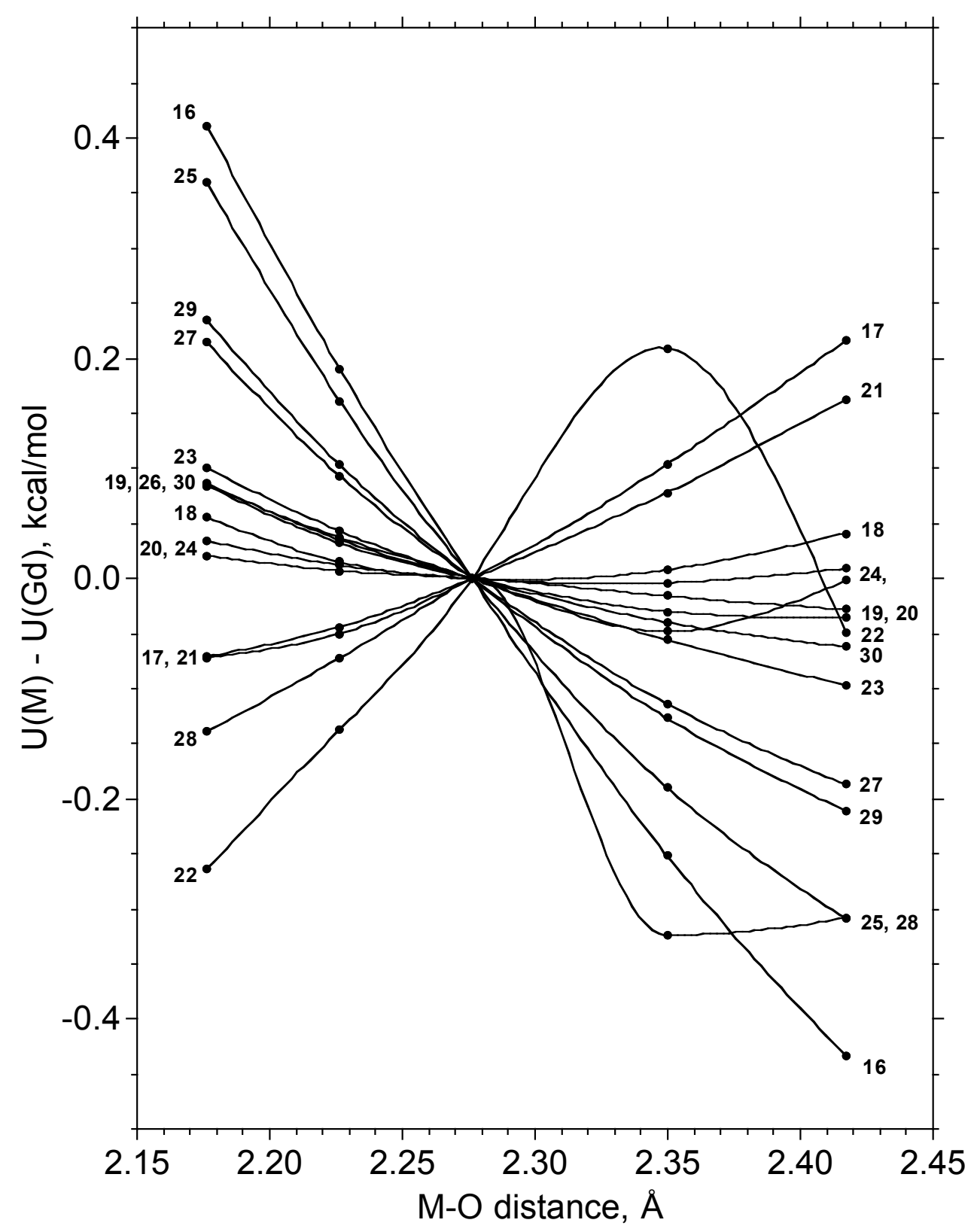

Figure S8. $\mathrm{U}(\mathrm{M})-\mathrm{U}(\mathrm{Gd})$ versus $\mathrm{M}-\mathrm{O}$ distance for $\mathbf{1 6}$ - $\mathbf{3 0}$. 


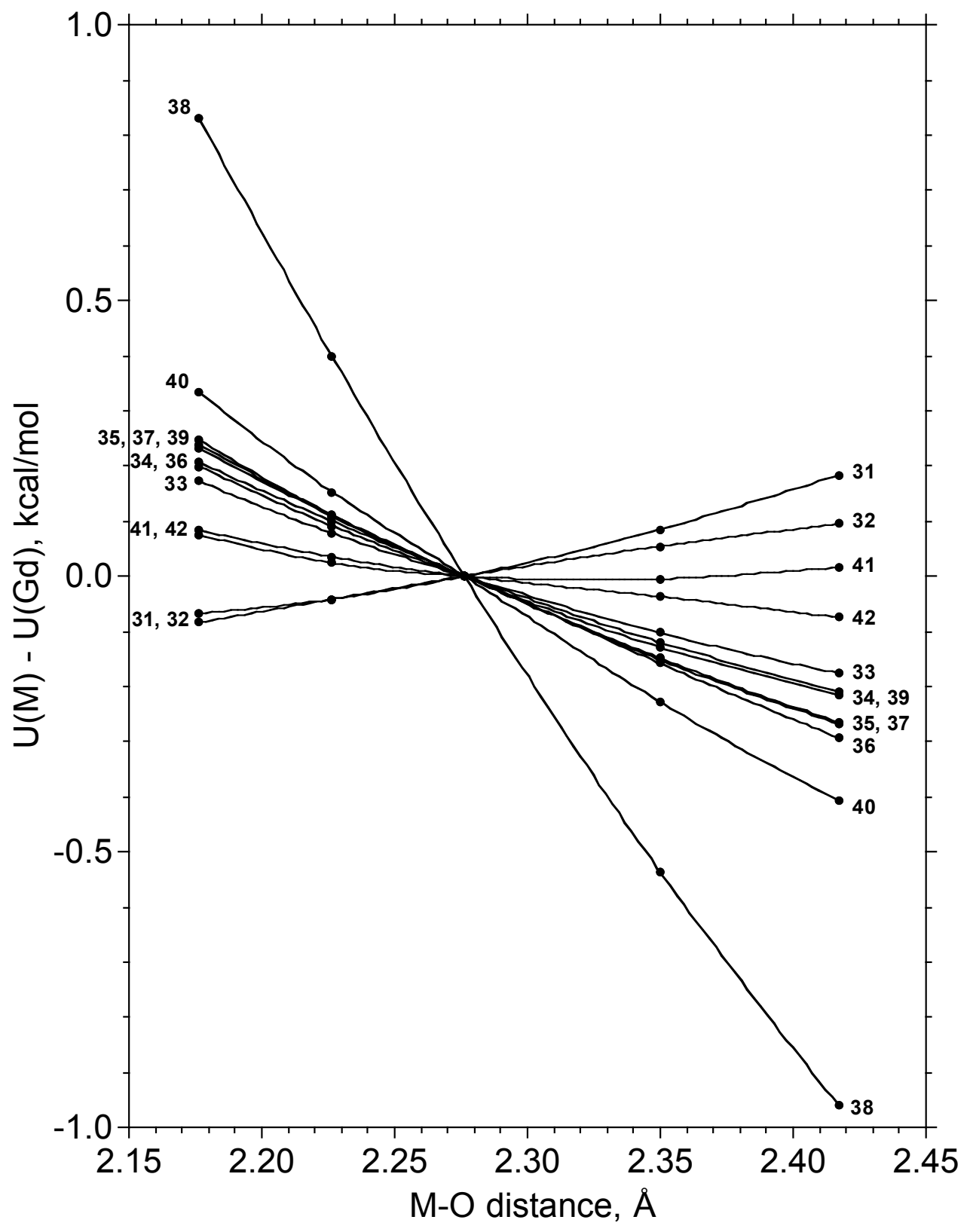

Figure S9. $U(M)-U(G d)$ versus $M-O$ distance for 31 - 42 . 


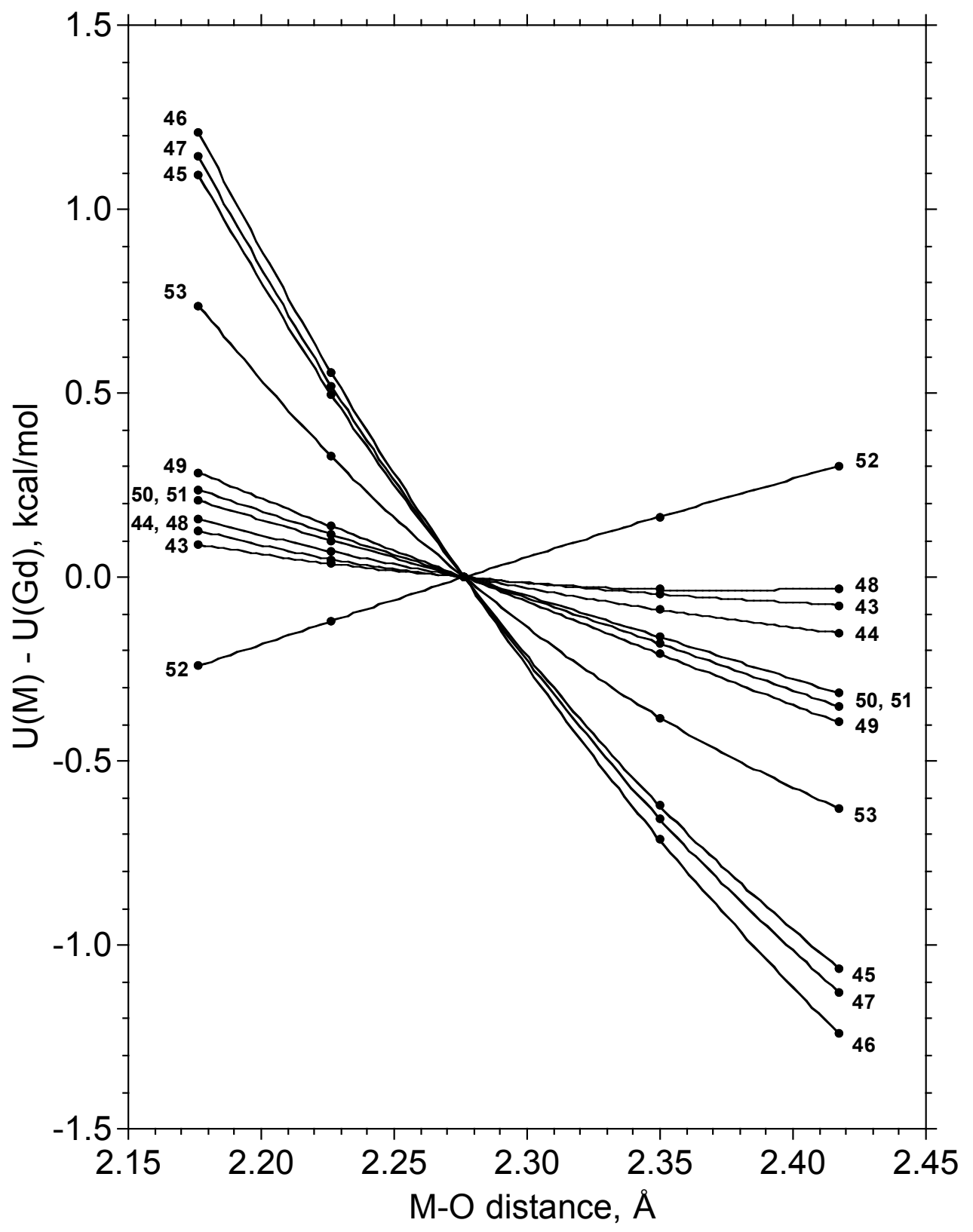

Figure S10. $\mathrm{U}(\mathrm{M})-\mathrm{U}(\mathrm{Gd})$ versus $\mathrm{M}-\mathrm{O}$ distance for $\mathbf{4 3}-\mathbf{5 2}$. 


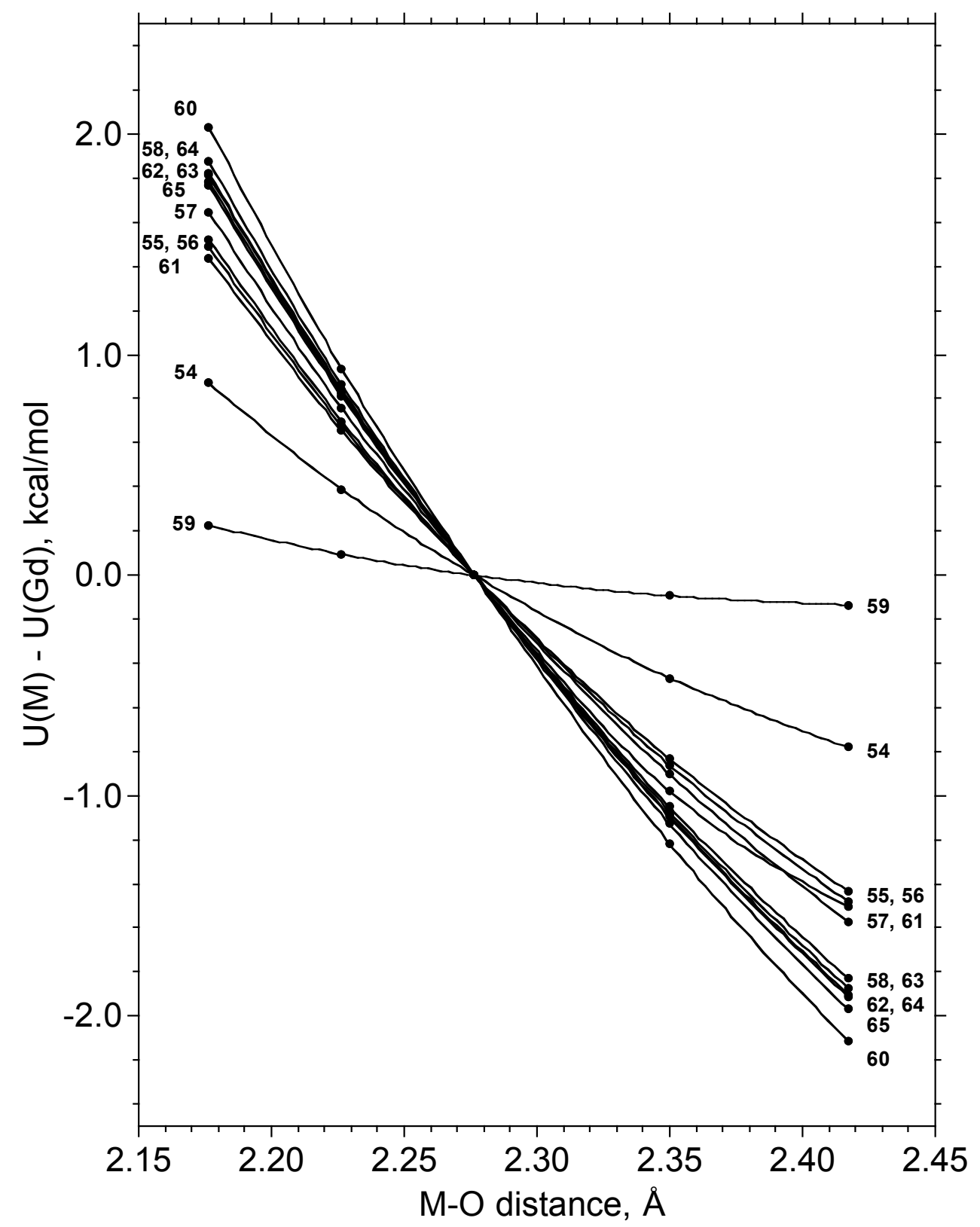

Figure S11. U(M) - U(Gd) versus M-O distance for $53-65$. 


\section{(7) Atomic coordinates and absolute energies (a.u.) for all DFT optimized geometries at the $B 3 L Y P / L C(G d) / 6-31+G(d)$ level.}

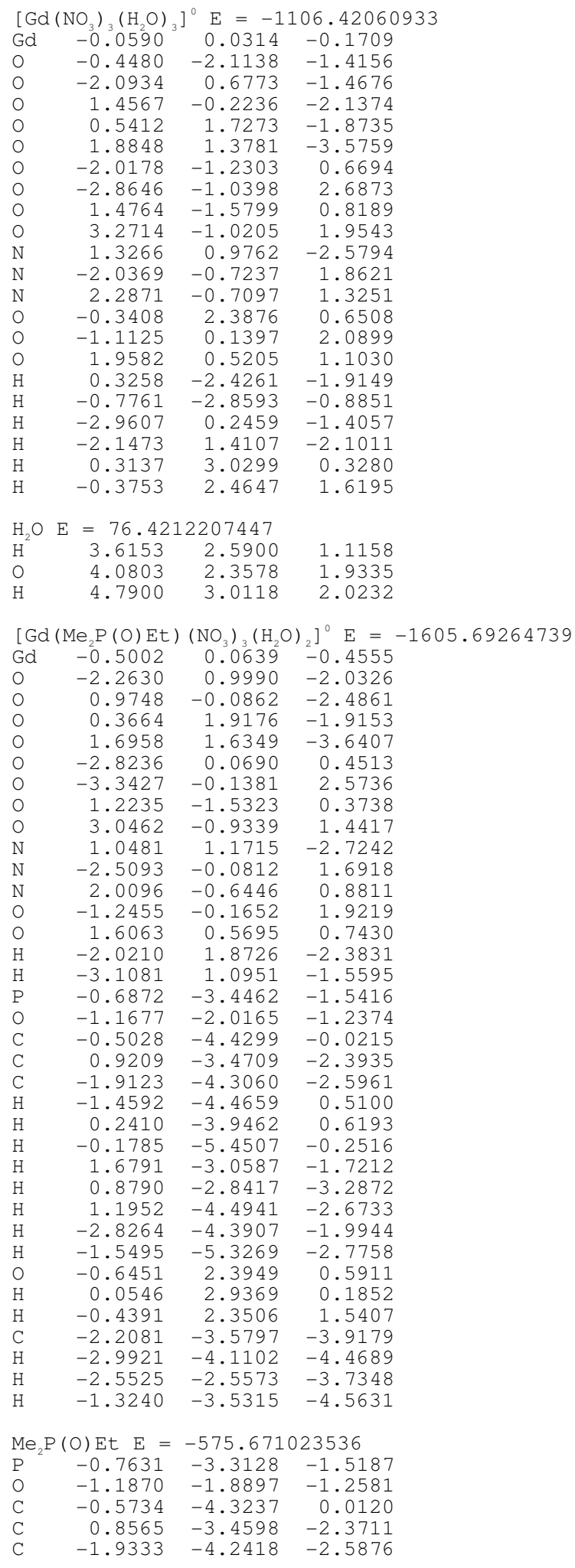




$\begin{array}{rrr}0.2771 & -3.8931 & 0.5569 \\ -0.2913 & -5.3482 & -0.2668 \\ 1.6275 & -2.9754 & -1.7626 \\ 0.8037 & -2.9381 & -3.3325 \\ 1.1389 & -4.5045 & -2.5447 \\ -2.9235 & -4.2515 & -2.1212 \\ -1.6053 & -5.2733 & -2.7594 \\ -2.0174 & -3.7267 & -3.5503 \\ -1.8253 & -4.3154 & 0.9025 \\ -1.6353 & -4.8562 & 1.8368 \\ -2.1159 & -3.2900 & 1.1520 \\ -2.6774 & -4.7978 & 0.4093\end{array}$

$\left[\mathrm{Gd}\left(\mathrm{Me}_{2} \mathrm{P}(\mathrm{O}) \mathrm{t}-\mathrm{Bu}\right)\left(\mathrm{NO}_{3}\right)_{3}\left(\mathrm{H}_{2} \mathrm{O}\right)_{2}\right]^{0} \mathrm{E}=-1684.31816718$

$\mathrm{Gd} \quad-0.4970 \quad 0.0562 \quad-2.27090 .9727$

$-2.2709 \quad 0.9727-2.0370$

$\begin{array}{lll}0.9734 & -0.0967 & -2.4912\end{array}$

$0.3504 \quad 1.9073-1.9366$

$\begin{array}{lll}1.6807 & 1.6202-3.6605\end{array}$

$\begin{array}{lll}-2.8222 & 0.0530 & 0.4508\end{array}$

$-3.3386-0.1444 \quad 2.5747$

$\begin{array}{lll}1.2511 & -1.5067 & 0.3851\end{array}$

$\begin{array}{lll}3.0661 & -0.8713 & 1.4446\end{array}$

$\begin{array}{lll}1.0371 & 1.1595 & -2.7397\end{array}$

$\begin{array}{lll}-2.5059 & -0.0861 & 1.6921\end{array}$

$\begin{array}{lll}2.0248 & -0.6028 & 0.8825\end{array}$

$\begin{array}{lll}-1.2416 & -0.1578 & 1.9223\end{array}$

$\begin{array}{lll}1.6036 & 0.6040 & 0.7331\end{array}$

$\begin{array}{lll}-2.0237 & 1.8418 & -2.3955\end{array}$

$\begin{array}{lll}-3.1085 & 1.0814 & -1.5534\end{array}$

$\begin{array}{lll}-0.6941 & -3.4694 & -1.5142\end{array}$

$\begin{array}{lll}-1.1563 & -2.0314 & -1.2089\end{array}$

$\begin{array}{lll}-0.4488 & -4.4081 & 0.0295\end{array}$

$\begin{array}{lll}0.9119 & -3.4854 & -2.3752\end{array}$

$\begin{array}{lll}-1.9813 & -4.3127 & -2.5632\end{array}$

$\begin{array}{lll}-1.3797 & -4.4441 & 0.6024\end{array}$

$\begin{array}{lll}0.3061 & -3.8792 & 0.6201\end{array}$

$\begin{array}{lll}-0.1030 & -5.4275 & -0.1688\end{array}$

$\begin{array}{lll}1.6528 & -3.0341 & -1.7081\end{array}$

$0.8598-2.8800 \quad-3.2840$

$\begin{array}{lll}1.2231 & -4.5049 & -2.6237\end{array}$

$\begin{array}{lll}-0.6667 & 2.3986 & 0.5648\end{array}$

$\begin{array}{lll}0.0301 & 2.9394 & 0.1523\end{array}$

$\begin{array}{lll}-0.4586 & 2.3655 & 1.5144\end{array}$

$\begin{array}{lll}-2.1306 & -3.5094 & -3.8743\end{array}$

$\begin{array}{llll}-2.9357 & -3.9507 & -4.4751\end{array}$

$\begin{array}{lll}-2.3878 & -2.4636 & -3.6789\end{array}$

$\begin{array}{lll}-1.2183 & -3.5331 & -4.4809\end{array}$

$\begin{array}{lll}-3.3182 & -4.2863 & -1.7894\end{array}$

$\begin{array}{lll}-4.1078 & -4.7099 & -2.4226\end{array}$

$\begin{array}{lll}-3.2781 & -4.8855 & -0.8726\end{array}$

$\begin{array}{lll}-3.6084 & -3.2657 & -1.5213\end{array}$

$\begin{array}{lll}-1.5777 & -5.7681 & -2.8762\end{array}$

$\begin{array}{lll}-2.3585 & -6.2305 & -3.4929\end{array}$

$\begin{array}{lll}-0.6404 & -5.8293 & -3.4407\end{array}$

$\begin{array}{lll}-1.4769 & -6.3773 & -1.9708\end{array}$

$\begin{array}{lrrr}\mathrm{Me}_{2} \mathrm{P}(\mathrm{O}) \mathrm{t}-\mathrm{Bu} E & =-654.295772587 \\ \mathrm{P} & -0.7329 & -3.3298 & -1.4987 \\ \mathrm{O} & -1.1462 & -1.9063 & -1.2144 \\ \mathrm{C} & -0.5108 & -4.3366 & 0.0693 \\ \mathrm{C} & 0.8403 & -3.4400 & -2.4456 \\ \mathrm{C} & -1.9403 & -4.2246 & -2.5591 \\ \mathrm{H} & 1.6482 & -2.9653 & -1.8808 \\ \mathrm{H} & 0.7071 & -2.8806 & -3.3778 \\ \mathrm{H} & 1.1226 & -4.4702 & -2.6869 \\ \mathrm{H} & -2.9161 & -4.2578 & -2.0655 \\ \mathrm{H} & -1.6213 & -5.2434 & -2.8025 \\ \mathrm{H} & -2.0522 & -3.6560 & -3.4885 \\ \mathrm{C} & -1.8531 & -4.3153 & 0.8320 \\ \mathrm{H} & -1.7292 & -4.8070 & 1.8061 \\ \mathrm{H} & -2.1938 & -3.2896 & 1.0065 \\ \mathrm{H} & -2.6423 & -4.8527 & 0.2927 \\ \mathrm{C} & -0.0887 & -5.7905 & -0.2166 \\ \mathrm{H} & 0.0336 & -6.3293 & 0.7325 \\ \mathrm{H} & -0.8391 & -6.3338 & -0.8027 \\ \mathrm{H} & 0.8691 & -5.8485 & -0.7470 \\ \mathrm{C} & 0.5652 & -3.6321 & 0.9241 \\ \mathrm{H} & 0.6503 & -4.1372 & 1.8954 \\ \mathrm{H} & 1.5538 & -3.6648 & 0.4507 \\ \mathrm{H} & 0.3056 & -2.5840 & 1.1044 \\ & & & \end{array}$




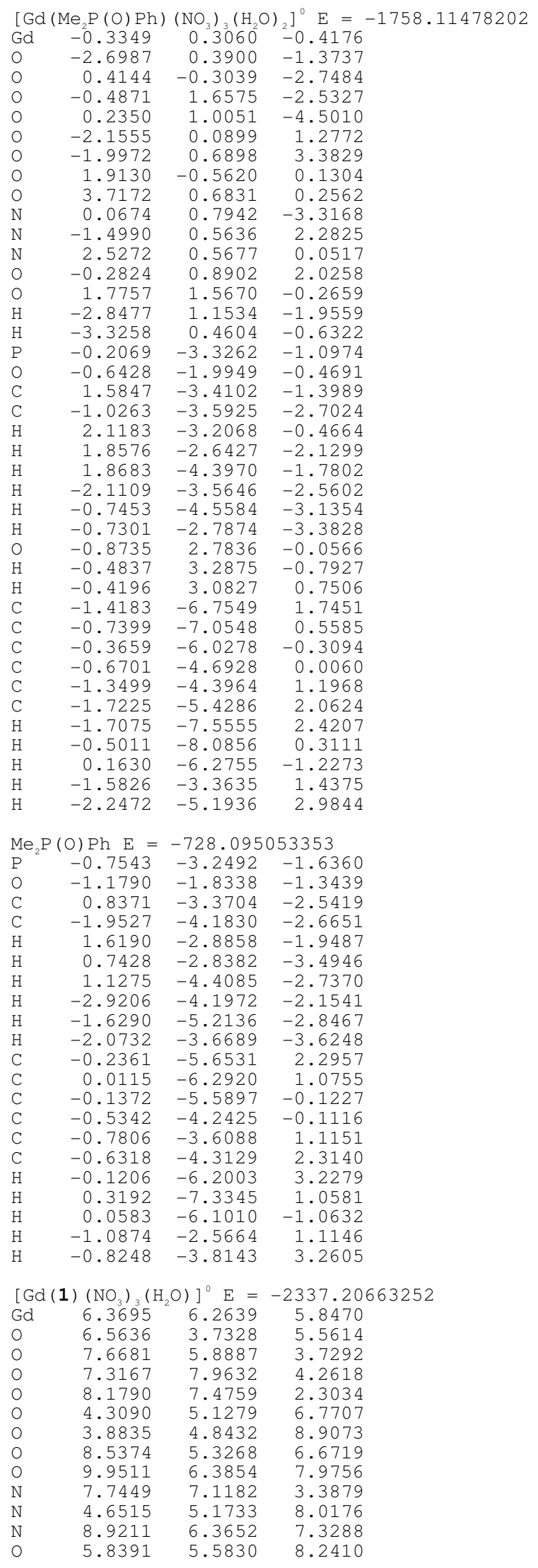




$\begin{array}{rrr}8.1332 & 7.3764 & 7.2470 \\ 7.4745 & 3.4995 & 5.8168 \\ 5.9743 & 3.2486 & 6.1624 \\ 7.0462 & 10.3997 & 6.5062 \\ 3.3922 & 6.1908 & 3.3947 \\ 5.3906 & 9.7223 & 6.8425 \\ 4.7269 & 6.3576 & 4.1196 \\ 3.5931 & 5.0578 & 1.9750 \\ 2.1233 & 5.3846 & 4.4344 \\ 5.2998 & 8.2155 & 6.5741 \\ 5.0482 & 10.0693 & 8.6012 \\ 7.7580 & 9.9256 & 7.1897 \\ 7.0615 & 11.4862 & 6.6479 \\ 7.3490 & 10.1498 & 5.4856 \\ 3.9408 & 4.0967 & 2.3684 \\ 4.3537 & 5.4395 & 1.2887 \\ 2.6535 & 4.9042 & 1.4337 \\ 2.5120 & 4.4225 & 4.7804 \\ 1.2036 & 5.2237 & 3.8606 \\ 1.9055 & 5.9883 & 5.3179 \\ 5.7710 & 9.5101 & 9.2042 \\ 4.0435 & 9.7275 & 8.8667 \\ 5.1407 & 11.1377 & 8.8241 \\ 2.8976 & 9.0404 & 3.6673 \\ 2.5045 & 8.8512 & 5.1448 \\ 2.7196 & 10.1304 & 5.9737 \\ 4.1577 & 10.6931 & 5.8554 \\ 4.5284 & 10.8574 & 4.3634 \\ 4.3404 & 9.5826 & 3.5204 \\ 4.6896 & 9.8593 & 2.0478 \\ 4.4575 & 8.6298 & 1.1604 \\ 3.0191 & 8.1127 & 1.3051 \\ 2.6414 & 7.7876 & 2.7721 \\ 1.5612 & 7.5754 & 2.8027 \\ 2.8458 & 7.2500 & 0.6518 \\ 2.3251 & 8.8939 & 0.9611 \\ 5.1789 & 7.8473 & 1.4319 \\ 4.6532 & 8.8791 & 0.1099 \\ 4.0730 & 10.6951 & 1.6780 \\ 5.7366 & 10.1785 & 1.9749 \\ 5.0338 & 8.8195 & 3.8866 \\ 5.5576 & 11.2195 & 4.2511 \\ 3.8820 & 11.6515 & 3.9573 \\ 4.1813 & 11.6886 & 6.3257 \\ 2.0349 & 10.9148 & 5.6190 \\ 2.4499 & 9.9416 & 7.0203 \\ 3.0966 & 8.0476 & 5.5909 \\ 1.4464 & 8.5614 & 5.2129 \\ 2.2256 & 9.8127 & 3.2512 \\ & & \end{array}$

S-94 


\begin{tabular}{|c|c|c|c|}
\hline C & -2.7101 & 1.3669 & 0.1460 \\
\hline $\mathrm{P}$ & 1.9055 & 1. 4342 & -0.7093 \\
\hline $\mathrm{P}$ & -3.0683 & -0.0988 & 1.1875 \\
\hline 0 & 0.5499 & 2.0942 & -0.8336 \\
\hline $\mathrm{C}$ & 3.1568 & 2.1751 & -1.8341 \\
\hline $\mathrm{C}$ & 2.6794 & 1.6742 & 0.9428 \\
\hline 0 & -2.8962 & 0.1595 & 2.6663 \\
\hline $\mathrm{C}$ & -4.7982 & -0.5309 & 0.7311 \\
\hline $\mathrm{H}$ & -3.3372 & 2.1830 & 0.5232 \\
\hline $\mathrm{H}$ & -2.9567 & 1.1921 & -0.9070 \\
\hline $\mathrm{H}$ & -1.6627 & 1.6750 & 0.2121 \\
\hline $\mathrm{H}$ & 3.2622 & 3.2310 & -1.5624 \\
\hline $\mathrm{H}$ & 2.8139 & 2.1240 & -2.8713 \\
\hline $\mathrm{H}$ & 4.1348 & 1.6876 & -1.7511 \\
\hline $\mathrm{H}$ & 2.7745 & 2.7514 & 1.1179 \\
\hline $\mathrm{H}$ & 3.6735 & 1.2161 & 0.9963 \\
\hline $\mathrm{H}$ & 2.0486 & 1.2550 & 1.7302 \\
\hline $\mathrm{H}$ & -5.4475 & 0.3267 & 0.9369 \\
\hline $\mathrm{H}$ & -5.1322 & -1.3703 & 1.3500 \\
\hline $\mathrm{H}$ & -4.8942 & -0.8032 & -0.3264 \\
\hline $\mathrm{C}$ & 0.6639 & -1.1659 & -0.5084 \\
\hline $\mathrm{C}$ & 0.3538 & -0.9264 & 0.9797 \\
\hline $\mathrm{C}$ & -0.8112 & -1.7980 & 1.4639 \\
\hline $\mathrm{C}$ & -2.0933 & -1.5974 & 0.6283 \\
\hline $\mathrm{C}$ & -1.7955 & -1.7216 & -0.8834 \\
\hline $\mathrm{C}$ & -0.5867 & -0.9050 & -1.3846 \\
\hline $\mathrm{C}$ & -0.3065 & -1.2214 & -2.8650 \\
\hline $\mathrm{C}$ & 0.9269 & -0.4775 & -3.3951 \\
\hline $\mathrm{C}$ & 2.1562 & -0.7685 & -2.5212 \\
\hline $\mathrm{C}$ & 1.9248 & -0.4131 & -1.0325 \\
\hline $\mathrm{H}$ & 2.7915 & -0.7776 & -0.4589 \\
\hline $\mathrm{H}$ & 3.0438 & -0.2604 & -2.9152 \\
\hline $\mathrm{H}$ & 2.3774 & -1.8455 & -2.5708 \\
\hline $\mathrm{H}$ & 0.7216 & 0.6017 & -3.4091 \\
\hline $\mathrm{H}$ & 1.1325 & -0.7752 & -4.4318 \\
\hline $\mathrm{H}$ & -0.1504 & -2.3064 & -2.9809 \\
\hline $\mathrm{H}$ & -1.1882 & -0.9681 & -3.4700 \\
\hline $\mathrm{H}$ & -0.8141 & 0.1636 & -1.3122 \\
\hline $\mathrm{H}$ & -2.6804 & -1.4605 & -1.4815 \\
\hline $\mathrm{H}$ & -1.6002 & -2.7858 & -1.0920 \\
\hline $\mathrm{H}$ & -2.7836 & -2.4107 & 0.8987 \\
\hline $\mathrm{H}$ & -0.5202 & -2.8572 & 1.3894 \\
\hline $\mathrm{H}$ & -1.0247 & -1.5931 & 2.5179 \\
\hline $\mathrm{H}$ & 0.1038 & 0.1284 & 1.1457 \\
\hline $\mathrm{H}$ & 1.2440 & -1.1440 & 1.5870 \\
\hline $\mathrm{H}$ & 0.9119 & -2.2381 & -0.6141 \\
\hline & 2) $\left(\mathrm{NO}_{3}\right)_{3}(\mathrm{H}$ & O) $]^{0} E=$ & -2219.25004648 \\
\hline $\mathrm{Gd}$ & 7.9450 & 8.0744 & 7.7293 \\
\hline 0 & 8.2507 & 5.7825 & 6.6487 \\
\hline 0 & 10.1522 & 8.0005 & 6.5158 \\
\hline 0 & 8.4708 & 8.7563 & 5.3715 \\
\hline 0 & 10.4629 & 8.6875 & 4.4532 \\
\hline 0 & 6.3538 & 6.3163 & 8.5756 \\
\hline 0 & 4.8268 & 6.6517 & 10.1130 \\
\hline 0 & 9.3758 & 6.6260 & 9.1868 \\
\hline 0 & 10.6811 & 7.3800 & 10.7831 \\
\hline $\mathrm{N}$ & 9.7303 & 8.4899 & 5.4064 \\
\hline $\mathrm{N}$ & 5.7936 & 7.0351 & 9.4759 \\
\hline $\mathrm{N}$ & 9.8748 & 7.5766 & 9.8937 \\
\hline 0 & 6.3145 & 8.1978 & 9.6530 \\
\hline 0 & 9.4534 & 8.7550 & 9.5904 \\
\hline $\mathrm{H}$ & 9.1993 & 5.5696 & 6.6911 \\
\hline $\mathrm{H}$ & 7.8052 & 5.1544 & 7.2448 \\
\hline $\mathrm{P}$ & 4.9276 & 9.0730 & 5.5973 \\
\hline 0 & 5.8326 & 8.4856 & 6.6759 \\
\hline $\mathrm{C}$ & 5.5919 & 8.8172 & 3.9195 \\
\hline $\mathrm{C}$ & 3.2897 & 8.2670 & 5.6367 \\
\hline $\mathrm{P}$ & 7.3841 & 11.6002 & 8.7580 \\
\hline 0 & 7.8376 & 10.4215 & 7.8944 \\
\hline $\mathrm{C}$ & 7.2868 & 11.1900 & 10.5276 \\
\hline $\mathrm{C}$ & 8.5570 & 12.9885 & 8.6051 \\
\hline $\mathrm{C}$ & 5.5655 & 12.8460 & 6.8450 \\
\hline $\mathrm{C}$ & 4.0228 & 12.9289 & 6.7764 \\
\hline $\mathrm{C}$ & 3.8148 & 11.4091 & 6.9716 \\
\hline $\mathrm{C}$ & 4.5818 & 11.1470 & 8.2977 \\
\hline $\mathrm{C}$ & 5.7263 & 12.2265 & 8.2647 \\
\hline $\mathrm{C}$ & 5.8815 & 11.8069 & 5.7313 \\
\hline $\mathrm{C}$ & 4.6091 & 10.8823 & 5.7411 \\
\hline
\end{tabular}




\begin{tabular}{|c|c|c|c|}
\hline \multicolumn{4}{|r|}{3.7352} \\
\hline $\mathrm{H}$ & 4.9402 & 9.2635 & 3.1604 \\
\hline $\mathrm{H}$ & 6.5963 & 9.2458 & 3.8585 \\
\hline $\mathrm{H}$ & 3.4214 & 7.1946 & 5.4603 \\
\hline $\mathrm{H}$ & 2.8386 & 8.3965 & 6.6252 \\
\hline $\mathrm{H}$ & 2.6192 & 8.6777 & 4.8738 \\
\hline $\mathrm{H}$ & 8.2625 & 10.8097 & 10.8475 \\
\hline $\mathrm{H}$ & 7.0182 & 12.0713 & 11.1206 \\
\hline $\mathrm{H}$ & 6.5506 & 10.3955 & 10.6819 \\
\hline $\mathrm{H}$ & 9.5329 & 12.6614 & 8.9787 \\
\hline $\mathrm{H}$ & 8.6660 & 13.2676 & 7.5527 \\
\hline $\mathrm{H}$ & 8.2252 & 13.8598 & 9.1801 \\
\hline $\mathrm{H}$ & 6.1004 & 13.7918 & 6.7184 \\
\hline $\mathrm{H}$ & 3.5839 & 13.5323 & 7.5797 \\
\hline $\mathrm{H}$ & 3.6513 & 13.2997 & 5.8137 \\
\hline $\mathrm{H}$ & 2.7753 & 11.0696 & 6.9989 \\
\hline $\mathrm{H}$ & 3.9242 & 11.3132 & 9.1571 \\
\hline $\mathrm{H}$ & 4.9594 & 10.1248 & 8.3713 \\
\hline $\mathrm{H}$ & 5.5028 & 13.0159 & 8.9972 \\
\hline $\mathrm{H}$ & 5.9806 & 12.3088 & 4.7630 \\
\hline $\mathrm{H}$ & 6.8080 & 11.2597 & 5.9171 \\
\hline $\mathrm{H}$ & 4.0033 & 11.0977 & 4.8482 \\
\hline 2 & $=-1265$. & 4409581 & \\
\hline $\bar{P}$ & 2.3132 & -0.2805 & -0.2894 \\
\hline 0 & 1.5023 & -1.3194 & -1.0309 \\
\hline $\mathrm{C}$ & 3.5703 & -1.0160 & 0.8282 \\
\hline $\mathrm{C}$ & 3.2753 & 0.8089 & -1.4110 \\
\hline $\mathrm{P}$ & -2.5116 & -1.1215 & 0.5285 \\
\hline 0 & -3.4196 & -1.3066 & 1.7218 \\
\hline $\mathrm{C}$ & -1.2930 & -2.4753 & 0.3272 \\
\hline $\mathrm{C}$ & -3.4049 & -1.0936 & -1.0781 \\
\hline $\mathrm{C}$ & -0.8770 & 0.9484 & 1.8206 \\
\hline $\mathrm{C}$ & -0.4021 & 2.3491 & 1.3657 \\
\hline $\mathrm{C}$ & 0.3317 & 1.8224 & 0.1091 \\
\hline $\mathrm{C}$ & -0.7836 & 1.0102 & -0.6139 \\
\hline $\mathrm{C}$ & -1.7011 & 0.5442 & 0.5693 \\
\hline $\mathrm{C}$ & 0.4679 & 0.1716 & 1.8923 \\
\hline $\mathrm{C}$ & 1.3500 & 0.8648 & 0.7908 \\
\hline $\mathrm{H}$ & 4.2284 & -1.6630 & 0.2387 \\
\hline $\mathrm{H}$ & 4.1735 & -0.2511 & 1.3300 \\
\hline $\mathrm{H}$ & 3.0721 & -1.6329 & 1.5829 \\
\hline $\mathrm{H}$ & 3.9744 & 0.1924 & -1.9859 \\
\hline $\mathrm{H}$ & 2.5950 & 1.2987 & -2.1149 \\
\hline $\mathrm{H}$ & 3.8376 & 1.5735 & -0.8636 \\
\hline $\mathrm{H}$ & -1.8286 & -3.3518 & -0.0556 \\
\hline $\mathrm{H}$ & -0.4675 & -2.2237 & -0.3473 \\
\hline $\mathrm{H}$ & -0.8918 & -2.7371 & 1.3112 \\
\hline $\mathrm{H}$ & -3.9694 & -2.0266 & -1.1806 \\
\hline $\mathrm{H}$ & -4.1165 & -0.2611 & -1.0891 \\
\hline $\mathrm{H}$ & -2.7190 & -0.9961 & -1.9271 \\
\hline $\mathrm{H}$ & -1.4558 & 0.9028 & 2.7459 \\
\hline $\mathrm{H}$ & -1.2299 & 3.0299 & 1.1352 \\
\hline $\mathrm{H}$ & 0.2691 & 2.8405 & 2.0811 \\
\hline $\mathrm{H}$ & 0.8027 & 2.5813 & -0.5235 \\
\hline $\mathrm{H}$ & -1.3397 & 1.6529 & -1.3058 \\
\hline $\mathrm{H}$ & -0.3677 & 0.1813 & -1.1924 \\
\hline $\mathrm{H}$ & -2.5989 & 1.1798 & 0.5855 \\
\hline $\mathrm{H}$ & 0.9258 & 0.2860 & 2.8811 \\
\hline $\mathrm{H}$ & 0.3386 & -0.8992 & 1.7198 \\
\hline $\mathrm{H}$ & 2.1137 & 1.4871 & 1.2803 \\
\hline & 23) $\left(\mathrm{NO}_{3}\right)_{3}$ & $\left.\left.\mathrm{H}_{2} \mathrm{O}\right)\right]^{\circ} \mathrm{E}=$ & -2141.83766590 \\
\hline GC & -1.0456 & -0.9803 & -0.5583 \\
\hline 0 & -3.5519 & -0.5799 & -0.2925 \\
\hline O & -0.9191 & 1.4055 & -0.5737 \\
\hline 0 & -2.2852 & -0.4443 & -2.6602 \\
\hline 0 & -0.1968 & -0.9701 & -2.9018 \\
\hline $\mathrm{O}$ & -1.3961 & -0.4519 & -4.6667 \\
\hline 0 & -0.7717 & -0.3047 & 1.8786 \\
\hline 0 & -1.7770 & -1.1673 & 3.6287 \\
\hline 0 & -2.0242 & -3.1920 & -1.1328 \\
\hline 0 & -1.0760 & -5.1150 & -0.6617 \\
\hline $\mathrm{N}$ & -1.2958 & -0.6193 & -3.4653 \\
\hline $\mathrm{N}$ & -1.5853 & -1.1515 & 2.4227 \\
\hline $\mathrm{N}$ & -1.0555 & -3.8996 & -0.6705 \\
\hline 0 & 1.2864 & -0.6574 & -0.3346 \\
\hline 0 & -2.1560 & -1.9509 & 1.6140 \\
\hline 0 & -0.0547 & -3.2225 & -0.2188 \\
\hline $\mathrm{P}$ & 2.5449 & -0.6898 & 0.5265 \\
\hline
\end{tabular}




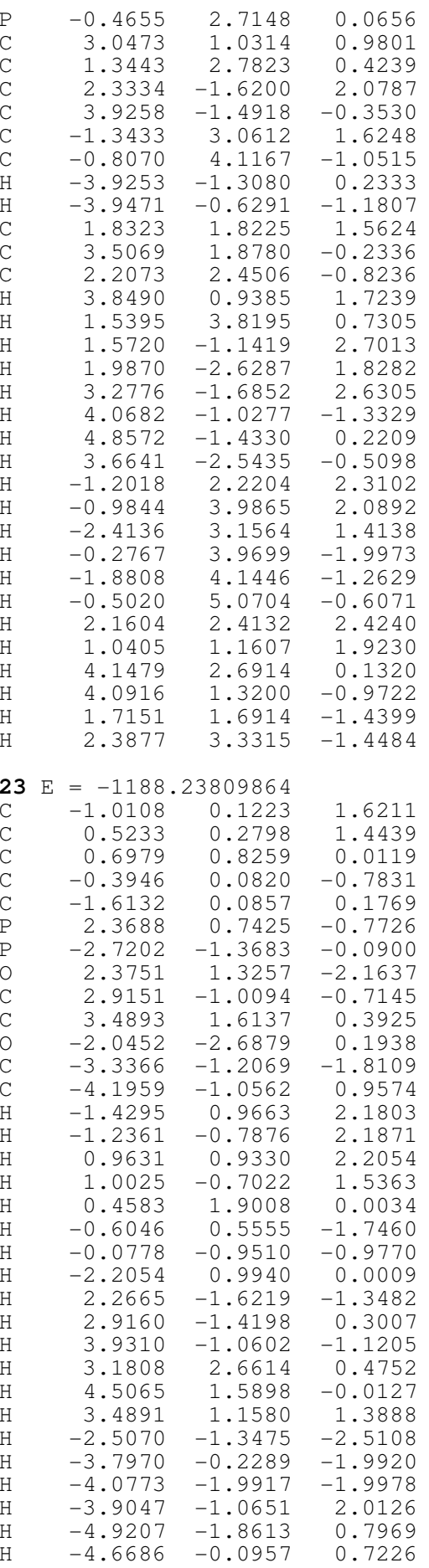

$\begin{array}{lrrr}{\left[\mathrm{Gd}(31)\left(\mathrm{NO}_{3}\right)_{3}\left(\mathrm{H}_{2} \mathrm{O}\right)\right]^{0} \mathrm{E}=} & -2256.17343449 \\ \mathrm{Gd} & 7.9616 & 7.9361 & 7.8738 \\ \mathrm{O} & 7.5917 & 5.5646 & 6.9499 \\ \mathrm{O} & 9.4341 & 7.9184 & 5.8550 \\ \mathrm{O} & 8.9740 & 9.8854 & 6.6478 \\ \mathrm{O} & 10.1238 & 9.7065 & 4.7863 \\ \mathrm{O} & 5.8160 & 6.8592 & 8.6957 \\ \mathrm{O} & 5.3461 & 6.4193 & 10.7975 \\ \mathrm{O} & 9.7390 & 6.2915 & 8.4824 \\ \mathrm{O} & 11.5239 & 6.7618 & 9.6693 \\ \mathrm{~N} & 9.5401 & 9.1873 & 5.7226 \\ \mathrm{~N} & 6.1445 & 6.7830 & 9.9494\end{array}$




\begin{tabular}{|c|c|c|}
\hline \\
\hline 10.4911 & 7.1092 & 9.1301 \\
\hline 7.3437 & 7.1140 & 10.2164 \\
\hline 10.0651 & 8.3225 & 9.1652 \\
\hline 8.3942 & 5.0469 & 7.1398 \\
\hline 6.8819 & 5.1965 & 7.5046 \\
\hline 4.9752 & 8.5299 & 5.5238 \\
\hline 6.3376 & 8.3884 & 6.19 \\
\hline 4.8116 & 7.3819 & 4.11 \\
\hline 3.5883 & 8.2111 & 6.65 \\
\hline 7.6850 & 11.2440 & \\
\hline 7.2865 & 9.8772 & 9.02 \\
\hline 6.66 & 11.6449 & 11.0 \\
\hline 9.4280 & 11.3372 & 10. \\
\hline 4.9176 & 6.3548 & 4.4 \\
\hline 5.6152 & 7.5762 & \\
\hline 3.8425 & 7.4913 & 3. \\
\hline 3.7379 & 7.2426 & 7.1 \\
\hline 2.6331 & 8.2201 & 6.12 \\
\hline 3.5751 & 8.9788 & 7.43 \\
\hline 6.7911 & 10.8506 & 11.78 \\
\hline 5.6117 & 11.6854 & 10.74 \\
\hline 6.9597 & 12.6034 & 11.47 \\
\hline 9.6236 & 10.58 & 10.8 \\
\hline 9.6664 & 12.33 & 10.4 \\
\hline 10.0659 & 11.10 & 9. \\
\hline 826 & 13.18 & \\
\hline 3.7698 & $12.9^{7}$ & 7.3 \\
\hline 3.6810 & 12.02 & \\
\hline 4.8168 & 11.3008 & \\
\hline 6.0341 & 11.5462 & 6. \\
\hline 6.1252 & 12.4626 & 7. \\
\hline 4.7426 & 10.2296 & 4. \\
\hline 7.4387 & 12.5958 & 8 . \\
\hline 5.0386 & 13.9147 & 8.7 \\
\hline 2.8902 & 13.54 & 7. \\
\hline 2.7290 & 11.85 & 5. \\
\hline 6.92 & 10.99 & 6. \\
\hline 3.7827 & 10.25 & \\
\hline 5.5360 & 10.3 & \\
\hline 8.2808 & 12.5 & \\
\hline 7.5238 & 13.55 & \\
\hline$=-1$ & 70510 & \\
\hline 2.6361 & -1.2445 & -0.15 \\
\hline 1.5537 & -2.2514 & -0.45 \\
\hline 2.8588 & -0.9164 & 1.63 \\
\hline 4.2936 & -1.7357 & -0.7 \\
\hline-3.2822 & -1.3210 & -0.4 \\
\hline-4.6713 & -1.6719 & -0.96 \\
\hline-3.1642 & -1.0644 & 1 . \\
\hline-2.0277 & -2.5952 & -0 . \\
\hline 3.0642 & -1.8641 & 2 . \\
\hline 1.9348 & -0.49 & 2 . \\
\hline 3.6840 & -0.22 & 1. \\
\hline 4.5824 & -2.68 & -0 . \\
\hline 5.0539 & -0.9783 & -0 . \\
\hline 4.2459 & -1.8923 & -1.844 \\
\hline-3.4747 & -1.9888 & 1.8 \\
\hline-3.8515 & -0.2665 & 1.6 \\
\hline-2.1486 & -0.8059 & 1. \\
\hline-2.3322 & -3.5284 & -0 \\
\hline-1.0142 & -2.3369 & -0.5 \\
\hline-2.0253 & -2.7677 & -1 \\
\hline-1.5330 & 1.97 & 0. \\
\hline-0.3 & 2.54 & 0 . \\
\hline 0.882 & 2.05 & 0 . \\
\hline 0.9 & 0 . & -0 \\
\hline-0.1 & 0 . & -1 \\
\hline-1 . & 0.90 & -0 \\
\hline 2.3 & & -0 \\
\hline-2.6 & 0.2687 & -1 \\
\hline-2.50 & 2.3620 & 0 \\
\hline-0.452 & 3.3738 & 1. \\
\hline 1.7805 & 2.5019 & 0 \\
\hline-0.0974 & -0 . & -1 \\
\hline 2.3994 & 0.2507 & -2 \\
\hline 3.1513 & 1.0945 & -0 . \\
\hline-3 . & & -1 \\
\hline-2 & 0 . & -2 . \\
\hline
\end{tabular}




$\begin{array}{lrrr}{\left[\mathrm{Gd}(39)\left(\mathrm{NO}_{3}\right)_{3}\left(\mathrm{H}_{2} \mathrm{O}\right)\right]^{0} \mathrm{E}=} & -2025.10283342 \\ \mathrm{Gd} & 6.1055 & 6.0484 & 5.7291 \\ \mathrm{O} & 6.4082 & 4.2623 & 3.9415 \\ \mathrm{O} & 8.2130 & 6.4899 & 4.3267 \\ \mathrm{O} & 6.4233 & 7.5069 & 3.6461 \\ \mathrm{O} & 8.3245 & 7.8462 & 2.6044 \\ \mathrm{O} & 4.6935 & 4.0210 & 6.0414 \\ \mathrm{O} & 3.3350 & 3.7057 & 7.7350 \\ \mathrm{O} & 7.7669 & 4.3857 & 6.5194 \\ \mathrm{O} & 9.3145 & 4.8141 & 8.0175 \\ \mathrm{~N} & 7.6885 & 7.2964 & 3.4905 \\ \mathrm{~N} & 4.1888 & 4.3681 & 7.1745 \\ \mathrm{~N} & 8.3531 & 5.1661 & 7.3633 \\ \mathrm{O} & 4.6456 & 5.4649 & 7.6595 \\ \mathrm{O} & 7.8409 & 6.3419 & 7.4574 \\ \mathrm{H} & 7.3601 & 4.0655 & 3.8931 \\ \mathrm{H} & 5.9863 & 3.4700 & 4.3204 \\ \mathrm{P} & 5.4130 & 9.7117 & 6.3007 \\ \mathrm{O} & 5.8417 & 8.2687 & 6.5096 \\ \mathrm{C} & 5.1459 & 10.5528 & 7.8942 \\ \mathrm{C} & 6.6590 & 10.6750 & 5.3837 \\ \mathrm{P} & 3.2186 & 7.5406 & 3.8563 \\ \mathrm{O} & 3.9339 & 6.6198 & 4.8343 \\ \mathrm{C} & 3.4577 & 7.0682 & 2.1146 \\ \mathrm{C} & 1.4207 & 7.5302 & 4.1678 \\ \mathrm{H} & 6.0753 & 10.5100 & 8.4711 \\ \mathrm{H} & 4.3707 & 10.0232 & 8.4572 \\ \mathrm{H} & 4.8514 & 11.5990 & 7.7587 \\ \mathrm{H} & 7.5966 & 10.6564 & 5.9493 \\ \mathrm{H} & 6.3427 & 11.7147 & 5.2451 \\ \mathrm{H} & 6.8418 & 10.2114 & 4.4096 \\ \mathrm{H} & 3.0835 & 6.0497 & 1.9677 \\ \mathrm{H} & 4.5289 & 7.0862 & 1.8928 \\ \mathrm{H} & 2.9270 & 7.7475 & 1.4383 \\ \mathrm{H} & 1.0561 & 6.5026 & 4.0697 \\ \mathrm{H} & 0.8850 & 8.1725 & 3.4605 \\ \mathrm{H} & 1.2147 & 7.8625 & 5.1900 \\ \mathrm{C} & 3.7672 & 9.2997 & 3.9648 \\ \mathrm{C} & 3.8131 & 9.8922 & 5.3945 \\ \mathrm{H} & 4.7463 & 9.3429 & 3.4758 \\ \mathrm{H} & 3.0765 & 9.8874 & 3.3462 \\ \mathrm{H} & 3.6039 & 10.9691 & 5.3501 \\ \mathrm{H} & 3.0420 & 9.4556 & 6.0416 \\ & & & \end{array}$




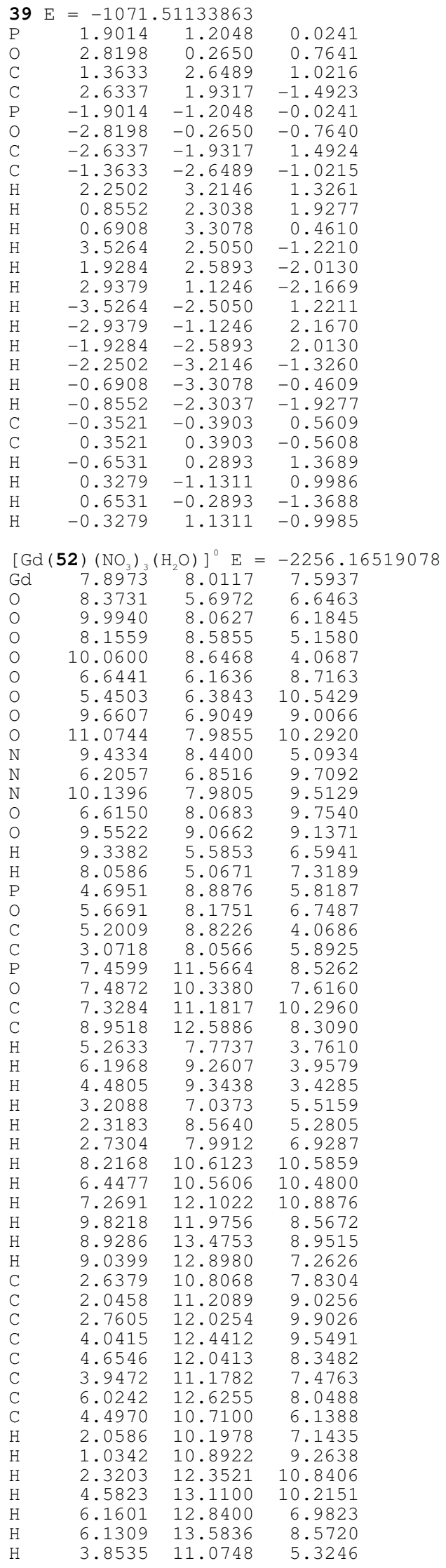




\begin{tabular}{|c|c|c|c|}
\hline $\mathrm{H}$ & 5.4987 & 11.1058 & 5.9516 \\
\hline & \multicolumn{3}{|c|}{$E=-1302.57194717$} \\
\hline $\mathrm{P}$ & -1.6483 & -2.2080 & 0.3201 \\
\hline 0 & -0.3774 & -2.8474 & 0.8322 \\
\hline C & -2.5800 & -3.2786 & -0.8421 \\
\hline $\mathrm{C}$ & -2.8345 & -1.7988 & 1.6570 \\
\hline $\mathrm{P}$ & 2.0818 & -0.4930 & -1.8753 \\
\hline O & 0.9275 & -0.7056 & -2.8286 \\
\hline $\mathrm{C}$ & 3.3732 & -1.7892 & -2.0048 \\
\hline $\mathrm{C}$ & 2.9586 & 1.0926 & -2.1565 \\
\hline $\mathrm{H}$ & -2.8101 & -4.2270 & -0.3452 \\
\hline $\mathrm{H}$ & -1.9520 & -3.4874 & -1.7144 \\
\hline $\mathrm{H}$ & -3.5128 & -2.8110 & -1.1761 \\
\hline $\mathrm{H}$ & -3.0629 & -2.7119 & 2.2167 \\
\hline $\mathrm{H}$ & -3.7654 & -1.3745 & 1.2641 \\
\hline $\mathrm{H}$ & -2.3734 & -1.0795 & 2.3408 \\
\hline $\mathrm{H}$ & 3.7324 & -1.8297 & -3.0386 \\
\hline $\mathrm{H}$ & 2.9309 & -2.7588 & -1.7535 \\
\hline $\mathrm{H}$ & 4.2199 & -1.5980 & -1.3363 \\
\hline $\mathrm{H}$ & 3.3052 & 1.1221 & -3.1949 \\
\hline $\mathrm{H}$ & 3.8174 & 1.2134 & -1.4867 \\
\hline $\mathrm{H}$ & 2.2630 & 1.9229 & -2.0002 \\
\hline $\mathrm{C}$ & -1.5585 & 1.5359 & 0.5897 \\
\hline $\mathrm{C}$ & -1.0377 & 2.6288 & 1.2848 \\
\hline $\mathrm{C}$ & 0.3350 & 2.6942 & 1.5254 \\
\hline $\mathrm{C}$ & 1.1593 & 1.6661 & 1.0643 \\
\hline $\mathrm{C}$ & 0.6508 & 0.5610 & 0.3633 \\
\hline $\mathrm{C}$ & -0.7429 & 0.4937 & 0.1207 \\
\hline $\mathrm{C}$ & 1.6129 & -0.5216 & -0.0846 \\
\hline $\mathrm{C}$ & -1.3863 & -0.6480 & -0.6411 \\
\hline $\mathrm{H}$ & -2.6282 & 1.4920 & 0.3942 \\
\hline $\mathrm{H}$ & -1.6989 & 3.4191 & 1.6310 \\
\hline $\mathrm{H}$ & 0.7628 & 3.5334 & 2.0676 \\
\hline $\mathrm{H}$ & 2.2283 & 1.7138 & 1.2623 \\
\hline $\mathrm{H}$ & 2.5442 & -0.4440 & 0.4914 \\
\hline $\mathrm{H}$ & 1.1976 & -1.5178 & 0.1181 \\
\hline $\mathrm{H}$ & -2.3683 & -0.3311 & -1.0152 \\
\hline 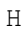 & -0.7851 & -0.9159 & -1.5200 \\
\hline & $54)\left(\mathrm{NO}_{3}\right)_{3}$ & $\left.\left.\mathrm{H}_{2} \mathrm{O}\right)\right]^{0} \mathrm{E}=$ & -2101.30500480 \\
\hline $\mathrm{Gc}$ & 8.0486 & 8.0755 & 7.8468 \\
\hline 0 & 8.3132 & 6.2242 & 6.1445 \\
\hline 0 & 10.3453 & 8.1549 & 6.7655 \\
\hline 0 & 8.8579 & 9.4864 & 5.9168 \\
\hline 0 & 10.9042 & 9.5005 & 5.1224 \\
\hline O & 6.3003 & 6.2897 & 8.0134 \\
\hline 0 & 4.4950 & 6.4189 & 9.2526 \\
\hline O & 9.2850 & 6.2170 & 8.9263 \\
\hline $\mathrm{O}$ & 10.5669 & 6.4737 & 10.6908 \\
\hline $\mathrm{N}$ & 10.0759 & 9.0622 & 5.9023 \\
\hline $\mathrm{N}$ & 5.5924 & 6.8458 & 8.9268 \\
\hline $\mathrm{N}$ & 9.8028 & 6.9320 & 9.8655 \\
\hline 0 & 6.1035 & 7.8977 & 9.4555 \\
\hline 0 & 9.4445 & 8.1692 & 9.8621 \\
\hline $\mathrm{H}$ & 9.2370 & 5.9192 & 6.1666 \\
\hline $\mathrm{H}$ & 7.7649 & 5.4736 & 6.4332 \\
\hline $\mathrm{P}$ & 7.3052 & 11.6756 & 8.2608 \\
\hline C & 8.2008 & 12.6395 & 6.9864 \\
\hline $\mathrm{C}$ & 7.1124 & 13.5013 & 6.3189 \\
\hline C & 5.8309 & 12.6465 & 6.1348 \\
\hline $\mathrm{C}$ & 5.6631 & 11.6829 & 7.3698 \\
\hline $\mathrm{C}$ & 5.8463 & 11.7860 & 4.8474 \\
\hline $\mathrm{P}$ & 5.0789 & 10.0484 & 6.6782 \\
\hline $\mathrm{C}$ & 4.7631 & 10.7018 & 4.9943 \\
\hline 0 & 7.8803 & 10.3225 & 8.6411 \\
\hline $\mathrm{C}$ & 7.0932 & 12.7110 & 9.7449 \\
\hline $\mathrm{C}$ & 3.5470 & 9.5599 & 7.5282 \\
\hline 0 & 6.1199 & 8.9414 & 6.6673 \\
\hline $\mathrm{H}$ & 8.6395 & 11.9102 & 6.2954 \\
\hline $\mathrm{H}$ & 9.0150 & 13.2287 & 7.4196 \\
\hline $\mathrm{H}$ & 6.8883 & 14.3730 & 6.9467 \\
\hline $\mathrm{H}$ & 7.4569 & 13.8911 & 5.3541 \\
\hline $\mathrm{H}$ & 4.9564 & 13.3072 & 6.0892 \\
\hline $\mathrm{H}$ & 4.9045 & 12.0533 & 8.0686 \\
\hline $\mathrm{H}$ & 6.8241 & 11.3053 & 4.7165 \\
\hline $\mathrm{H}$ & 5.6709 & 12.4132 & 3.9663 \\
\hline $\mathrm{H}$ & 3.7547 & 11.1318 & 4.9347 \\
\hline $\mathrm{H}$ & 4.8470 & 9.8964 & 4.2586 \\
\hline $\mathrm{H}$ & 6.4068 & 12.2136 & 10.4378 \\
\hline
\end{tabular}

S-101 


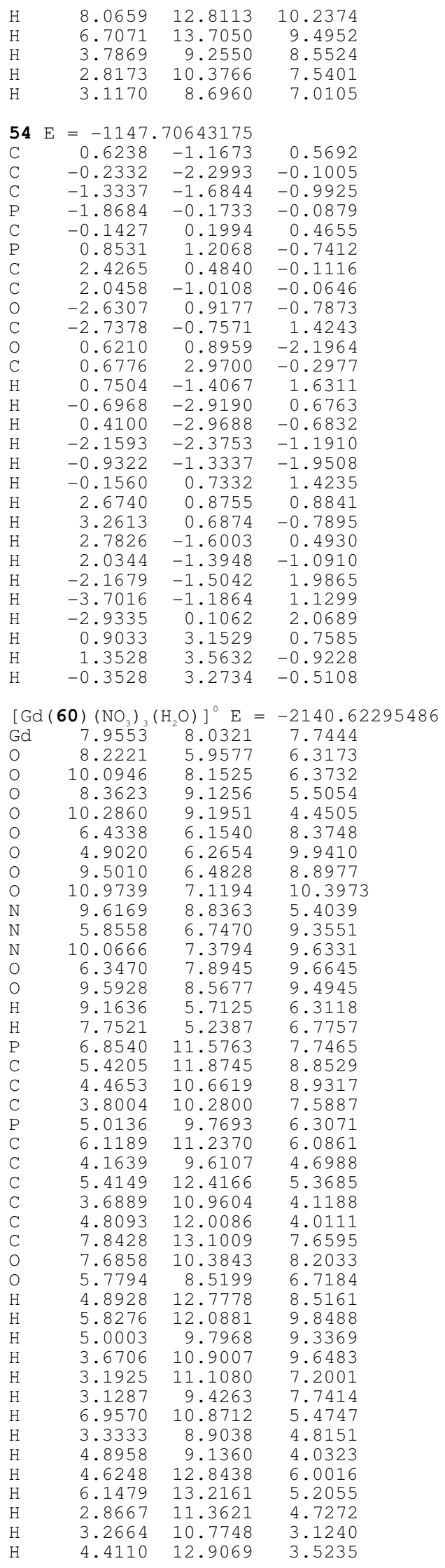




$\begin{array}{rrr}5.6079 & 11.6275 & 3.3596 \\ 7.2342 & 13.9730 & 7.4011 \\ 8.3096 & 13.2615 & 8.6370 \\ 8.6366 & 12.9724 & 6.9163 \\ & & \\ -11187.02447268 & \\ -1.5347 & -0.5132 & -0.4567 \\ -0.8339 & -2.2168 & -0.3257 \\ -0.1666 & -2.5025 & 1.0336 \\ 1.1136 & -1.6743 & 1.2644 \\ 0.7616 & 0.1249 & 1.4676 \\ -0.0739 & 0.6082 & -0.1324 \\ 2.3841 & 0.9951 & 1.3980 \\ 0.9066 & 0.7230 & -1.3321 \\ 3.0669 & 1.0345 & 0.0212 \\ 2.1459 & 1.5962 & -1.0698 \\ -1.9606 & -0.3201 & -2.2345 \\ -2.7113 & -0.2656 & 0.4444 \\ -0.0131 & 0.4376 & 2.7168 \\ -0.1234 & -2.3893 & -1.1467 \\ -1.6793 & -2.8988 & -0.4810 \\ -0.8799 & -2.3085 & 1.8430 \\ 0.0887 & -3.5685 & 1.0835 \\ 1.8304 & -1.8359 & 0.4469 \\ 1.6039 & -1.9883 & 2.1944 \\ -0.5202 & 1.5917 & 0.0743 \\ 3.0297 & 0.5386 & 2.1595 \\ 2.1683 & 2.0139 & 1.7469 \\ 1.2442 & -0.2767 & -1.6422 \\ 0.3616 & 1.1389 & -2.1878 \\ 3.4008 & 0.0272 & -0.2671 \\ 3.9752 & 1.6471 & 0.0915 \\ 2.7076 & 1.6910 & -2.0083 \\ 1.8264 & 2.6121 & -0.7943 \\ -1.1465 & -0.5887 & -2.9146 \\ -2.8209 & -0.9691 & -2.4313 \\ -2.2692 & 0.7133 & -2.4246\end{array}$




\section{(7) Full citation for reference 35(a).}

M. J. Frisch, G. W. Trucks, H. B. Schlegel, G. E. S.; M. A. Robb, J. R. Cheeseman, G. Scalmani, V. Barone, B. M.; G. A. Petersson, H. Nakatsuji, M. Caricato, X. Li, H. P. H.; A. F. Izmaylov, J. Bloino, G. Zheng, J. L. Sonnenberg, M. H.; M. Ehara, K. Toyota, R. Fukuda, J. Hasegawa, M. Ishida, T. N.; Y. Honda, O. Kitao, H. Nakai, T. Vreven, J. A. M. J.; J. E. Peralta, F. Ogliaro, M. Bearpark, J. J. Heyd, E. B.; K. N. Kudin, V. N. Staroverov, T. Keith, R. Kobayashi, J. N.; K. Raghavachari, A. Rendell, J. C. Burant, S. S. Iyengar, J. T.; M. Cossi, N. Rega, J. M. Millam, M. Klene, J. E. Knox, J. B. C.; V. Bakken, C. Adamo, J. Jaramillo, R. Gomperts, R. E. S.; O. Yazyev, A. J. Austin, R. Cammi, C. Pomelli, J. W. O.; R. L. Martin, K. Morokuma, V. G. Zakrzewski, G. A. V.; P. Salvador, J. J. Dannenberg, S. Dapprich, A. D. D.; O. Farkas, J. B. Foresman, J. V. Ortiz, J. C.; Fox, D. J. F. Gaussian 09, Revision C.01; Gaussian, Inc.: Wallingford, CT, 2010. 